\title{
Rhodium-Catalyzed Enantioselective Anti-Markovnikov Hydroformylation of $\alpha$-Substituted Acryl Acid Derivatives
}

Shuailong Li, Zhuangxing, Li, Cai You, Xiuxiu Li, Jiaxin Yang, Hui Lv, Xumu Zhang

\section{Table of Contents}

1. General Information $\quad$ S2

2. Ligand screening in the asymmetric hydroformylation (AHF) of 1a $\quad$ S2

3. General procedure for AHF $\quad$ S3

4. Procedures for gram-scale AHF of $\mathbf{1 u}$ and the synthesis of $\mathbf{4 a} \quad \mathrm{S} 11$

5. Procedures for gram-scale AHF of $\mathbf{1 c}$ and the synthesis of $\mathbf{4 b} \quad \mathrm{S} 12$

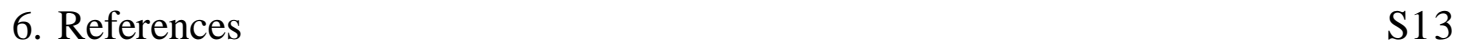

$\begin{array}{lr}\text { 7. NMR spectra } & \mathrm{S} 14\end{array}$

$\begin{array}{lr}\text { 8. HPLC spectra } & \text { S39 }\end{array}$ 
1. General Information. All reactions and manipulations that were sensitive to moisture or air were performed in a ragon-filled glovebox or using standard Schlenk techniques, unless otherwise noted. Solvents were dried with standard procedures, degassed with $\mathrm{N}_{2}$ and transferred by syringe. NMR spectra were recorded on Bruker

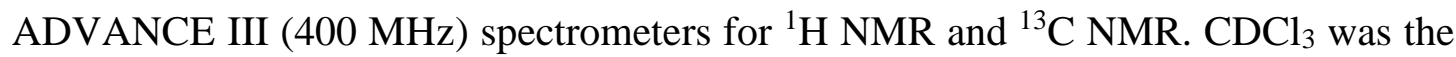
solvent used for the NMR analysis, with tetramethylsilane as the internal standard. Chemical shifts were reported upfield to TMS (0.00 ppm) for ${ }^{1} \mathrm{H}$ NMR and relative to $\mathrm{CDCl}_{3}\left(77.0 \mathrm{ppm}\right.$ ) for ${ }^{13} \mathrm{C}$ NMR. Optical rotation was determined using a Perkin Elmer 343 polarimeter. HPLC analysis was conducted on an Agilent 1260 Series instrument. Thin layer chromatography (TLC) was performed on EM reagents $0.25 \mathrm{~mm}$ silica 60 F plates. All new products were further characterized by HRMS. A positive ion mass spectrum of sample was acquired on a Thermo LTQ-FT mass spectrometer with an electrospray ionization source.

\section{Ligand screening in the asymmetric hydroformylation (AHF) of 1 a}

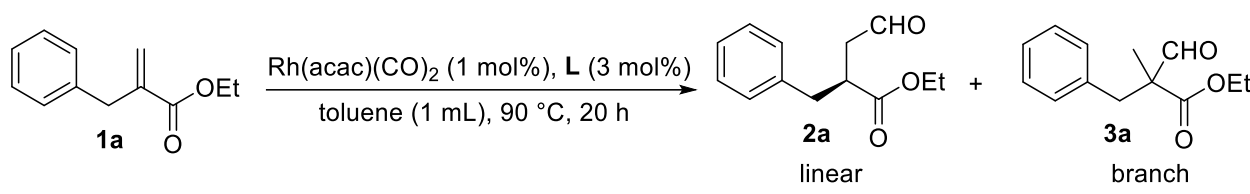

\begin{tabular}{|c|c|c|c|c|}
\hline entry & ligand & $\mathrm{CO} / \mathrm{H}_{2}$ (bar) & conv. $(\%)$ & $\mathbf{2 a}$ ee $(\%)$ \\
\hline 1 & $(S)$-BINAP & $5 / 5$ & 11 & $10(R)$ \\
\hline 2 & Xuphos & $5 / 5$ & 6 & 35 \\
\hline 3 & $(S)$-Segphos & $5 / 5$ & 34 & 32 \\
\hline 4 & $(S, S)$-Me-Duphos & $5 / 5$ & 14 & 53 \\
\hline 5 & Walphos & $5 / 5$ & 64 & 55 \\
\hline 6 & $(S)-S y n p h o s$ & $5 / 5$ & 28 & $33(R)$ \\
\hline 7 & $(S)$-Binapine & $5 / 5$ & $>99$ & - \\
\hline 8 & $\left(R_{\mathrm{c}}, S_{\mathrm{p}}\right)$-Duanphos & $5 / 5$ & $>99$ & $76(R)$ \\
\hline 9 & $(S, R)$-YanPhos & $5 / 5$ & 55 & 20 \\
\hline 10 & $(S, S)$-YanPhos & $5 / 5$ & $>99$ & 54 \\
\hline 11 & $(S, S)$-DTB-YanPhos & $5 / 5$ & $>99$ & 86 \\
\hline 12 & $(S, S)$-DTBM-YanPhos & $5 / 5$ & $>99$ & 90 \\
\hline 13 & $(S, S)$-DTBM-YanPhos & $10 / 10$ & 90 & 90 \\
\hline 14 & $(S, S)$-DTBM-YanPhos & $2.5 / 2.5$ & $>99$ & 90 \\
\hline $15^{b}$ & $(S, S)$-DTBM-YanPhos & $2.5 / 2.5$ & $>99$ & 90 \\
\hline $16^{c}$ & $(S, S)$-DTBM-YanPhos & $2.5 / 2.5$ & 88 & 90 \\
\hline $17^{d}$ & $(S, S)$-DTBM-YanPhos & $2.5 / 2.5$ & $>99$ & 90 \\
\hline $18^{e}$ & $(S, S)$-DTBM-YanPhos & $2.5 / 2.5$ & 93 & 86 \\
\hline
\end{tabular}




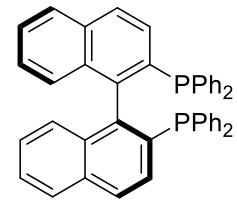

(S)-BINAP

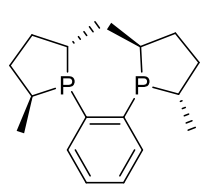

(S,S)-Me-DuPhos

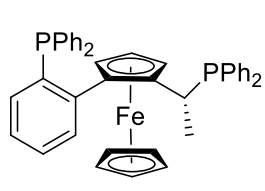

Walphos

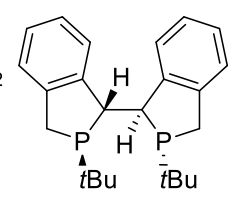

$(R \mathrm{c}, S p)$-DuanPhos

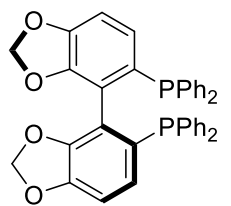

(S)-SegPhos

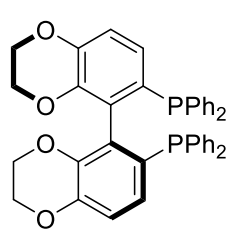

(S)-Synphos

(S)-Binapine

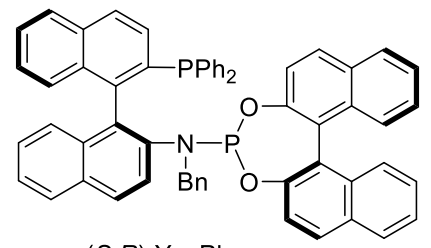

$(S, R)$-YanPhos

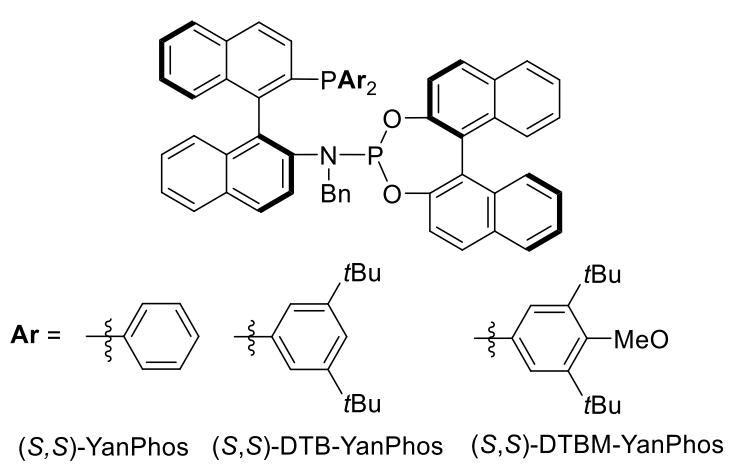

\section{General procedure for AHF}

In a glovebox filled with argon, to a $5 \mathrm{ml}$ vial equipped with a magnetic bar was added $(S, S)$-DTBM-YanPhos $(0.004 \mathrm{mmol})$ and $\mathrm{Rh}(\mathrm{acac})(\mathrm{CO})_{2}(0.002 \mathrm{mmol}$ in $1 \mathrm{~mL}$ toluene $)$. After stirring for $10 \mathrm{~min}$, the mixture was charged to substrate $(0.2 \mathrm{mmol})$. The vial was transferred into an autoclave and taken out of the glovebox. The argon gas was replacement with hydrogen gas for three times, and then hydrogen ( 2.5 bar) and carbon monoxide $\left(2.5\right.$ bar) were charged in sequence. The reaction mixture was stirred at $80^{\circ} \mathrm{C}$ (oil bath) for $20 \mathrm{~h}$. The reaction was cooled to room temperature and the pressure was carefully released in a well-ventilated hood. The solution was transferred into a solution of methyl 2-(triphenyl- $\lambda^{4}$-phosphanyl)acetate $(0.22 \mathrm{mmol})$ in $2 \mathrm{~mL}$ dichloromethane, the reaction mixture was stirred at $25{ }^{\circ} \mathrm{C}$ (oil bath) for $2 \mathrm{~h}$. The solution was concentrated and the ester was isolated by column chromatography. The enantiomeric excesses of $\mathbf{2 a - 2 f}, \mathbf{2 j - 2}$ and $\mathbf{2 n - 2} \mathbf{w}$ were determined by HPLC analysis using a chiral stationary phase after the above-mentioned Wittig reaction, the enantiomeric excesses of $\mathbf{2 g}-\mathbf{2} \mathbf{i}$ and $\mathbf{2} \mathbf{m}$ were determined by HPLC analysis using a chiral stationary phase after reduced by $\mathrm{NaBH}_{4}$.<smiles>CCOC(=O)C(CC=O)Cc1ccccc1</smiles>

ethyl $(\boldsymbol{S})$-2-benzyl-4-oxobutanoate (2a): ${ }^{1} 41.4 \mathrm{mg}$, colorless oil; isolated yield: $94 \%$; $90 \%$ ee; $[\alpha]_{\mathrm{D}}^{25}=-20.6\left(\mathrm{c}=1.0, \mathrm{CHCl}_{3}\right)$. Ref $1:[\alpha]_{\mathrm{D}}^{25}=+18\left(\mathrm{c}=0.91, \mathrm{CHCl}_{3},(\mathrm{R})\right)$. 
The enantiomeric excess was determined by HPLC on Chiralcel OD-H column, hexane: isopropanol $=98: 2$; flow rate $=1.0 \mathrm{~mL} / \mathrm{min}$; $\mathrm{UV}$ detection at $220 \mathrm{~nm} ; \mathrm{t}_{\mathrm{R}}=14.8 \mathrm{~min}$ (minor), $17.0 \mathrm{~min}$ (major). ${ }^{1} \mathrm{H}$ NMR $\left(400 \mathrm{MHz}, \mathrm{CDCl}_{3}\right) \delta 9.70(\mathrm{~s}, 1 \mathrm{H}), 7.31-7.28(\mathrm{~m}$, $2 \mathrm{H})$, 7.24-7.21 (m, 1H), 7.17-7.15 (m, 2H), $4.12(\mathrm{q}, \mathrm{J}=7.1 \mathrm{~Hz}, 2 \mathrm{H}), 3.20-3.13(\mathrm{~m}, 1 \mathrm{H})$, $3.07(\mathrm{dd}, \mathrm{J}=13.5,6.3 \mathrm{~Hz}, 1 \mathrm{H}), 2.87-2.73(\mathrm{~m}, 2 \mathrm{H}), 2.54-2.48(\mathrm{~m}, 1 \mathrm{H}), 1.20(\mathrm{t}, \mathrm{J}=7.1$ $\mathrm{Hz}, 3 \mathrm{H}) \mathrm{ppm} .{ }^{13} \mathrm{C} \mathrm{NMR}\left(101 \mathrm{MHz}, \mathrm{CDCl}_{3}\right) \delta 200.0,174.0,138.1,129.0,128.5,126.7$, $60.8,44.4,40.9,37.6,14.0 \mathrm{ppm}$.<smiles>COC(=O)[C@H](CC=O)Cc1ccccc1</smiles>

methyl (S)-2-benzyl-4-oxobutanoate (2b): ${ }^{2} 34.2 \mathrm{mg}$, colorless oil; isolated yield: 83\%; $89 \%$ ee; $[\alpha]_{\mathrm{D}}{ }^{25}=-24.7\left(\mathrm{c}=1.0, \mathrm{CHCl}_{3}\right)$. The enantiomeric excess was determined by HPLC on Chiralcel OD-H column, hexane: isopropanol $=98: 2$; flow rate $=1.0 \mathrm{~mL} / \mathrm{min}$; $\mathrm{UV}$ detection at $220 \mathrm{~nm}$; $\mathrm{t}_{\mathrm{R}}=18.1 \mathrm{~min}$ (minor), $20.3 \mathrm{~min}$ (major). ${ }^{1} \mathrm{H} \mathrm{NMR}(400 \mathrm{MHz}$, $\left.\mathrm{CDCl}_{3}\right) \delta 9.69(\mathrm{~s}, 1 \mathrm{H}), 7.32-7.28(\mathrm{~m}, 2 \mathrm{H}), 7.25-7.21(\mathrm{~m}, 1 \mathrm{H}), 7.16-7.14(\mathrm{~m}, 2 \mathrm{H}), 3.68$ (s, 3H), 3.22-3.15 (m, 1H), 3.08 (dd, J = 13.6, 6.1 Hz, 1H), 2.88-2.73 (m, 2H), $2.52(\mathrm{dd}$, $\mathrm{J}=18.4,4.5 \mathrm{~Hz}, 1 \mathrm{H}) \mathrm{ppm} .{ }^{13} \mathrm{C} \mathrm{NMR}\left(101 \mathrm{MHz}, \mathrm{CDCl}_{3}\right) \delta 200.0,174.5,138.0,128.9$, $128.6,126.8,52.0,44.3,40.7,37.5 \mathrm{ppm}$.<smiles>CCCOC(=O)[C@H](CC=O)Cc1ccccc1</smiles>

isopropyl (S)-2-benzyl-4-oxobutanoate (2c): $41.2 \mathrm{mg}$, colorless oil; isolated yield: $88 \% ; 91 \%$ ee; $[\alpha]_{\mathrm{D}}^{25}=-20.0\left(\mathrm{c}=1.0, \mathrm{CHCl}_{3}\right)$. The enantiomeric excess was determined by HPLC on Chiralcel OD-H column, hexane: isopropanol $=98: 2$; flow rate $=1.0$ $\mathrm{mL} / \mathrm{min}$; UV detection at $220 \mathrm{~nm}$; $\mathrm{t}_{\mathrm{R}}=11.7 \mathrm{~min}$ (major), $13.0 \mathrm{~min}$ (minor). ${ }^{1} \mathrm{H}$ NMR $\left(400 \mathrm{MHz}, \mathrm{CDCl}_{3}\right) \delta 9.70(\mathrm{~s}, 1 \mathrm{H}), 7.31-7.16(\mathrm{~m}, 5 \mathrm{H}), 5.02-4.93(\mathrm{~m}, 1 \mathrm{H}), 3.17-3.10(\mathrm{~m}$, $1 \mathrm{H}), 3.05(\mathrm{dd}, J=13.5,6.6 \mathrm{~Hz}, 1 \mathrm{H}), 2.85-2.72(\mathrm{~m}, 2 \mathrm{H}), 2.50(\mathrm{dd}, J=18.2,4.6 \mathrm{~Hz}, 1 \mathrm{H})$, $1.22(\mathrm{~d}, J=6.3 \mathrm{~Hz}, 3 \mathrm{H}), 1.13(\mathrm{~d}, J=6.3 \mathrm{~Hz}, 3 \mathrm{H}) \mathrm{ppm} .{ }^{13} \mathrm{C} \mathrm{NMR}\left(101 \mathrm{MHz}, \mathrm{CDCl}_{3}\right) \delta$ 200.1, 173.5, 138.1, 129.0, 128.5, 126.7, 68.2, 44.5, 41.1, 37.6, 21.7, 21.6 ppm. HRMS calculated $[\mathrm{M}+\mathrm{Na}]^{+}$for $\mathrm{C}_{14} \mathrm{H}_{18} \mathrm{NaO}_{3}=257.1148$, found: 257.1152 .<smiles>O=CCC(Cc1ccccc1)C(=O)OC(c1ccccc1)c1ccccc1</smiles>

benzhydryl (S)-2-benzyl-4-oxobutanoate (2d): $53.8 \mathrm{mg}$, colorless oil; isolated yield: $75 \% ; 90 \%$ ee; $[\alpha]_{\mathrm{D}}{ }^{25}=-4.4\left(\mathrm{c}=1.0, \mathrm{CHCl}_{3}\right)$. The enantiomeric excess was determined by HPLC on Chiralcel OD-H column, hexane: isopropanol $=98: 2$; flow rate $=1.0$ $\mathrm{mL} / \mathrm{min}$; $\mathrm{UV}$ detection at $220 \mathrm{~nm}$; $\mathrm{t}_{\mathrm{R}}=23.6 \mathrm{~min}$ (major), $27.1 \mathrm{~min}$ (minor). ${ }^{1} \mathrm{H} \mathrm{NMR}$ $\left(400 \mathrm{MHz}, \mathrm{CDCl}_{3}\right) \delta 9.67(\mathrm{~s}, 1 \mathrm{H}), 7.36-7.19(\mathrm{~m}, 13 \mathrm{H}), 7.09-7.07(\mathrm{~m}, 2 \mathrm{H}), 6.85(\mathrm{~s}, 1 \mathrm{H})$, $3.38-3.31(\mathrm{~m}, 1 \mathrm{H}), 3.09(\mathrm{dd}, J=13.7,6.6 \mathrm{~Hz}, 1 \mathrm{H}), 2.88-2.75(\mathrm{~m}, 2 \mathrm{H}), 2.54(\mathrm{dd}, J=$ 
18.4, $4.8 \mathrm{~Hz}, 1 \mathrm{H}) \mathrm{ppm} .{ }^{13} \mathrm{C} \mathrm{NMR}\left(101 \mathrm{MHz}, \mathrm{CDCl}_{3}\right) \delta 199.8,173.2,139.7,139.7$, $137.8,129.0,128.6,128.4,127.9,127.2,127.1,126.8,44.4,40.9,37.4$ ppm. HRMS calculated $[\mathrm{M}+\mathrm{Na}]^{+}$for $\mathrm{C}_{24} \mathrm{H}_{22} \mathrm{NaO}_{3}=381.1461$, found: 381.1459 .<smiles>O=CCC(Cc1ccccc1)C(=O)Oc1ccccc1</smiles>

phenyl (S)-2-benzyl-4-oxobutanoate (2e): $42.4 \mathrm{mg}$, colorless oil; isolated yield: 79\%; $88 \%$ ee; $[\alpha]_{\mathrm{D}}{ }^{25}=-26.1\left(\mathrm{c}=1.0, \mathrm{CHCl}_{3}\right)$. The enantiomeric excess was determined by HPLC on Chiralcel OD-H column, hexane: isopropanol $=98: 2$; flow rate $=1.0 \mathrm{~mL} / \mathrm{min}$; UV detection at $220 \mathrm{~nm}$; $\mathrm{t}_{\mathrm{R}}=19.7 \mathrm{~min}$ (major), $21.8 \mathrm{~min}$ (minor). ${ }^{1} \mathrm{H} \mathrm{NMR}(400 \mathrm{MHz}$, $\left.\mathrm{CDCl}_{3}\right) \delta 9.77(\mathrm{~s}, 1 \mathrm{H}), 7.36-7.32(\mathrm{~m}, 4 \mathrm{H}), 7.27-7.19(\mathrm{~m}, 4 \mathrm{H}), 6.96-6.94(\mathrm{~m}, 2 \mathrm{H}), 3.44-$ $3.37(\mathrm{~m}, 1 \mathrm{H}), 3.17(\mathrm{dd}, J=13.6,6.9 \mathrm{~Hz}, 1 \mathrm{H}), 3.03-2.90(\mathrm{~m}, 2 \mathrm{H}), 2.70(\mathrm{dd}, J=18.6,4.5$ $\mathrm{Hz}, 1 \mathrm{H}) \mathrm{ppm} .{ }^{13} \mathrm{C} \mathrm{NMR}\left(101 \mathrm{MHz}, \mathrm{CDCl}_{3}\right) \delta 199.7,172.9,150.5,137.7,129.4,129.1$, 128.7, 127.0, 125.9, 121.4, 44.8, 40.9, 37.7 ppm. HRMS calculated $[\mathrm{M}+\mathrm{Na}]^{+}$for $\mathrm{C}_{17} \mathrm{H}_{16} \mathrm{NaO}_{3}=291.0991$, found: 291.0993 .<smiles>O=CCC(Cc1ccccc1)C(=O)OCc1ccccc1</smiles>

benzyl (S)-2-benzyl-4-oxobutanoate (2f): $48.0 \mathrm{mg}$, colorless oil; isolated yield: $85 \%$; $88 \%$ ee; $[\alpha]_{\mathrm{D}}^{25}=-10.2\left(\mathrm{c}=1.0, \mathrm{CHCl}_{3}\right)$. The enantiomeric excess was determined by HPLC on Chiralcel OD-H column, hexane: isopropanol $=98: 2$; flow rate $=1.0 \mathrm{~mL} / \mathrm{min}$; $\mathrm{UV}$ detection at $220 \mathrm{~nm} ; \mathrm{t}_{\mathrm{R}}=22.8 \mathrm{~min}$ (minor), $24.9 \mathrm{~min}$ (major). ${ }^{1} \mathrm{H} \mathrm{NMR}(400 \mathrm{MHz}$, $\left.\mathrm{CDCl}_{3}\right) \delta 9.69(\mathrm{~s}, 1 \mathrm{H}), 7.35-7.21(\mathrm{~m}, 8 \mathrm{H}), 7.12-7.11(\mathrm{~m}, 2 \mathrm{H}), 5.10(\mathrm{~s}, 2 \mathrm{H}), 3.28-3.20$ (m, 1H), 3.07 (dd, $J=13.6,6.4 \mathrm{~Hz}, 1 \mathrm{H}), 2.89-2.75(\mathrm{~m}, 2 \mathrm{H}), 2.53(\mathrm{dd}, J=18.4,4.7 \mathrm{~Hz}$, $1 \mathrm{H}) \mathrm{ppm} .{ }^{13} \mathrm{C} \mathrm{NMR}\left(101 \mathrm{MHz}, \mathrm{CDCl}_{3}\right) \delta 199.9,173.9,137.9,135.6,129.0,128.6$, 128.5, 128.2, 128.2, 126.8, 66.7, 44.4, 40.8, $37.5 \mathrm{ppm}$. HRMS calculated $[\mathrm{M}+\mathrm{Na}]^{+}$for $\mathrm{C}_{18} \mathrm{H}_{18} \mathrm{NaO}_{3}=305.1148$, found: 305.1146 .<smiles>CCNC(=O)C(CC=O)Cc1ccccc1</smiles>

(S)-2-benzyl-N,N-diethyl-4-oxobutanamide (2g): $39.6 \mathrm{mg}$, light yellow oil; isolated yield: $80 \% ; 28 \%$ ee; $[\alpha]_{\mathrm{D}}{ }^{25}=-59.7\left(\mathrm{c}=1.0, \mathrm{CHCl}_{3}\right)$. The enantiomeric excess was determined by HPLC on Chiralcel OD-H column, hexane: isopropanol = 95:5; flow rate $=1.0 \mathrm{~mL} / \mathrm{min} ; \mathrm{UV}$ detection at $210 \mathrm{~nm} ; \mathrm{t}_{\mathrm{R}}=12.4 \mathrm{~min}$ (major), $19.8 \mathrm{~min}$ (minor). ${ }^{1} \mathrm{H}$ NMR $\left(400 \mathrm{MHz}, \mathrm{CDCl}_{3}\right) \delta 9.72(\mathrm{~s}, 1 \mathrm{H}), 7.30-7.17(\mathrm{~m}, 5 \mathrm{H}), 3.48-3.39(\mathrm{~m}, 1 \mathrm{H}), 3.35-$ $3.28(\mathrm{~m}, 1 \mathrm{H}), 3.24-3.07(\mathrm{~m}, 4 \mathrm{H}), 2.94(\mathrm{dd}, J=13.3,7.7 \mathrm{~Hz}, 1 \mathrm{H}), 2.68(\mathrm{dd}, J=13.3,7.4$ $\mathrm{Hz}, 1 \mathrm{H}), 2.54$ (dd, $J=18.5,4.2 \mathrm{~Hz}, 1 \mathrm{H}), 1.05(\mathrm{t}, J=7.1 \mathrm{~Hz}, 6 \mathrm{H}) \mathrm{ppm} .{ }^{13} \mathrm{C}$ NMR $(101$ $\left.\mathrm{MHz}, \mathrm{CDCl}_{3}\right) \delta 200.9,173.0,138.7,129.1,128.5,126.6,46.6,41.8,40.5,39.1,37.5$, 
14.0, 12.8 ppm. HRMS calculated $[\mathrm{M}+\mathrm{H}]^{+}$for $\mathrm{C}_{15} \mathrm{H}_{22} \mathrm{NO}_{2}=248.1645$, found: 248.1640 .<smiles>CCC(Cc1ccccc1)C(=O)N1CCCCC1</smiles>

(S)-3-benzyl-4-oxo-4-(piperidin-1-yl)butanal (2h): $31.1 \mathrm{mg}$, light yellow oil; isolated yield: $60 \% ; 75 \%$ ee; $[\alpha]_{\mathrm{D}}{ }^{25}=-55.9\left(\mathrm{c}=1.0, \mathrm{CHCl}_{3}\right)$. The enantiomeric excess was determined by HPLC on Chiralpak AD-H column, hexane: isopropanol = 95:5; flow rate $=1.0 \mathrm{~mL} / \mathrm{min} ; \mathrm{UV}$ detection at $220 \mathrm{~nm} ; \mathrm{t}_{\mathrm{R}}=17.3 \mathrm{~min}$ (major), $20.0 \mathrm{~min}$ (minor). ${ }^{1} \mathrm{H} \mathrm{NMR}\left(400 \mathrm{MHz}, \mathrm{CDCl}_{3}\right) \delta 9.72(\mathrm{~s}, 1 \mathrm{H}), 7.30-7.16(\mathrm{~m}, 5 \mathrm{H}), 3.55-3.44(\mathrm{~m}, 3 \mathrm{H}), 3.39$ $3.33(\mathrm{~m}, 1 \mathrm{H}), 3.21-3.12(\mathrm{~m}, 2 \mathrm{H}), 2.91(\mathrm{dd}, J=13.2,8.3 \mathrm{~Hz}, 1 \mathrm{H}), 2.67(\mathrm{dd}, J=13.2,7.1$ $\mathrm{Hz}, 1 \mathrm{H}), 2.53(\mathrm{dd}, J=18.6,3.9 \mathrm{~Hz}, 1 \mathrm{H}), 1.56-1.49$ (m, 4H), 1.40-1.33 (m, 1H), 1.07$1.02(\mathrm{~m}, 1 \mathrm{H}) \mathrm{ppm} .{ }^{13} \mathrm{C} \mathrm{NMR}\left(101 \mathrm{MHz}, \mathrm{CDCl}_{3}\right) \delta 200.9,172.0,138.7,129.0,128.5$, 126.6, 46.8, 46.6, 43.0, 38.9, 36.8, 25.9, 25.4, $24.4 \mathrm{ppm}$. HRMS calculated $[\mathrm{M}+\mathrm{H}]^{+}$for $\mathrm{C}_{16} \mathrm{H}_{22} \mathrm{NO}_{2}=260.1645$, found: 260.1641 .<smiles>CCC(Cc1ccccc1)C(=O)N1CCOCC1</smiles>

(S)-3-benzyl-4-morpholino-4-oxobutanal (2i): $40.2 \mathrm{mg}$, light yellow oil; isolated yield: $77 \% ; 86 \%$ ee; $[\alpha]_{\mathrm{D}}{ }^{25}=-70.5\left(\mathrm{c}=1.0, \mathrm{CHCl}_{3}\right)$. The enantiomeric excess was determined by HPLC on Chiralcel OD-H column, hexane: isopropanol = 90:10; flow rate $=1.0 \mathrm{~mL} / \mathrm{min}$; $\mathrm{UV}$ detection at $210 \mathrm{~nm} ; \mathrm{t}_{\mathrm{R}}=11.7 \mathrm{~min}$ (minor), $14.9 \mathrm{~min}$ (major). ${ }^{1} \mathrm{H}$ NMR $\left(400 \mathrm{MHz}, \mathrm{CDCl}_{3}\right) \delta 9.73(\mathrm{~s}, 1 \mathrm{H}), 7.31-7.15(\mathrm{~m}, 5 \mathrm{H}), 3.62-3.57(\mathrm{~m}, 2 \mathrm{H}), 3.55-$ $3.50(\mathrm{~m}, 1 \mathrm{H}), 3.48-3.36(\mathrm{~m}, 4 \mathrm{H}), 3.22(\mathrm{dd}, J=18.9,9.5 \mathrm{~Hz}, 1 \mathrm{H}), 3.10-3.04(\mathrm{~m}, 1 \mathrm{H})$, 2.93-2.84 (m, 2H), $2.72(\mathrm{dd}, J=13.1,6.1 \mathrm{~Hz}, 1 \mathrm{H}), 2.63(\mathrm{dd}, J=18.9,3.7 \mathrm{~Hz}, 1 \mathrm{H}) \mathrm{ppm}$. ${ }^{13} \mathrm{C} \mathrm{NMR}\left(101 \mathrm{MHz}, \mathrm{CDCl}_{3}\right) \delta 200.6,172.5,138.5,129.0,128.6,126.8,66.5,66.1$, 47.1, 46.1, 42.1, 39.1, 36.5 ppm. HRMS calculated $[\mathrm{M}+\mathrm{H}]^{+}$for $\mathrm{C}_{15} \mathrm{H}_{20} \mathrm{NO}_{3}=262.1438$, found: 262.1437 .<smiles>CCCOC(=O)C(CC=O)Cc1ccc(F)cc1</smiles>

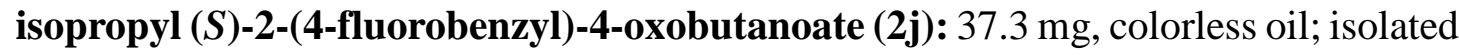
yield: $74 \% ; 90 \%$ ee; $[\alpha]_{\mathrm{D}}{ }^{25}=-18.8\left(\mathrm{c}=1.0, \mathrm{CHCl}_{3}\right)$. The enantiomeric excess was determined by HPLC on Chiralcel OD-H column, hexane: isopropanol = 98:2; flow rate $=1.0 \mathrm{~mL} / \mathrm{min} ; \mathrm{UV}$ detection at $220 \mathrm{~nm} ; \mathrm{t}_{\mathrm{R}}=8.7 \mathrm{~min}$ (minor), $10.7 \mathrm{~min}$ (major). ${ }^{1} \mathrm{H}$ NMR (400 MHz, $\left.\mathrm{CDCl}_{3}\right) \delta 9.72(\mathrm{~s}, 1 \mathrm{H}), 7.15-7.11(\mathrm{~m}, 2 \mathrm{H}), 7.01-6.95(\mathrm{~m}, 2 \mathrm{H}), 5.01-$ 4.92(m, 1H), 3.14-3.07 (m, 1H), 2.99 (dd, $J=13.7,7.0 \mathrm{~Hz}, 1 \mathrm{H}), 2.86-2.73(\mathrm{~m}, 2 \mathrm{H})$, $2.52(\mathrm{dd}, J=18.2,4.9 \mathrm{~Hz}, 1 \mathrm{H}), 1.21(\mathrm{~d}, J=6.3 \mathrm{~Hz}, 3 \mathrm{H}), 1.12(\mathrm{~d}, J=6.3 \mathrm{~Hz}, 3 \mathrm{H}) \mathrm{ppm}$. 
${ }^{13} \mathrm{C} \mathrm{NMR}\left(101 \mathrm{MHz}, \mathrm{CDCl}_{3}\right) \delta 199.9,173.4,161.7(\mathrm{~d}, J=244.8 \mathrm{~Hz}), 133.8(\mathrm{~d}, J=3.3$ $\mathrm{Hz}), 130.5(\mathrm{~d}, J=7.9 \mathrm{~Hz}), 115.3(\mathrm{~d}, J=21.3 \mathrm{~Hz}), 68.3,44.6,41.2(\mathrm{~d}, J=0.8 \mathrm{~Hz}), 36.8$, $21.6(\mathrm{~d}, J=8.1 \mathrm{~Hz})$ ppm. HRMS calculated $[\mathrm{M}+\mathrm{Na}]^{+}$for $\mathrm{C}_{14} \mathrm{H}_{17} \mathrm{FNaO}_{3}=275.1054$, found: 275.1069 .<smiles>CCCC(Cc1ccc(Cl)cc1)C(=O)OCC</smiles>

isopropyl (S)-2-(4-chlorobenzyl)-4-oxobutanoate (2k): $41.9 \mathrm{mg}$, colorless oil; isolated yield: $78 \% ; 90 \%$ ee; $[\alpha]_{\mathrm{D}}{ }^{25}=-20.9\left(\mathrm{c}=1.0, \mathrm{CHCl}_{3}\right)$. The enantiomeric excess was determined by HPLC on Chiralcel OD-H column, hexane: isopropanol = 98:2; flow rate $=1.0 \mathrm{~mL} / \mathrm{min} ; \mathrm{UV}$ detection at $220 \mathrm{~nm} ; \mathrm{t}_{\mathrm{R}}=9.5 \mathrm{~min}$ (minor), $11.8 \mathrm{~min}$ (major). ${ }^{1} \mathrm{H}$ NMR (400 MHz, $\left.\mathrm{CDCl}_{3}\right) \delta 9.71(\mathrm{~s}, 1 \mathrm{H}), 7.27-7.25(\mathrm{~m}, 2 \mathrm{H}), 7.12-7.10(\mathrm{~m}, 2 \mathrm{H}), 5.01-$ $4.92(\mathrm{~m}, 1 \mathrm{H}), 3.14-3.07(\mathrm{~m}, 1 \mathrm{H}), 2.99(\mathrm{dd}, J=13.6,7.0 \mathrm{~Hz}, 1 \mathrm{H}), 2.82(\mathrm{dd}, J=18.2,8.7$ $\mathrm{Hz}, 1 \mathrm{H}), 2.75(\mathrm{dd}, J=13.6,7.9 \mathrm{~Hz}, 1 \mathrm{H}), 2.51(\mathrm{dd}, J=18.2,4.8 \mathrm{~Hz}, 1 \mathrm{H}), 1.21(\mathrm{~d}, J=$ $6.3 \mathrm{~Hz}, 3 \mathrm{H}), 1.13(\mathrm{~d}, J=6.3 \mathrm{~Hz}, 3 \mathrm{H}) \mathrm{ppm} .{ }^{13} \mathrm{C} \mathrm{NMR}\left(101 \mathrm{MHz}, \mathrm{CDCl}_{3}\right) \delta 199.8,173.3$, 136.6, 132.5, 130.3, 128.6, 68.4, 44.5, 40.9, 36.9, 21.6, 21.6 ppm. HRMS calculated $[\mathrm{M}+\mathrm{Na}]^{+}$for $\mathrm{C}_{14} \mathrm{H}_{17} \mathrm{ClNaO}_{3}=291.0758$, found: 291.0762 .<smiles>CCCC(Cc1ccc(Br)cc1)C(=O)OCC</smiles>

isopropyl (S)-2-(4-bromobenzyl)-4-oxobutanoate (21): $48.2 \mathrm{mg}$, colorless oil; isolated yield: $77 \% ; 90 \%$ ee; $[\alpha]_{\mathrm{D}}{ }^{25}=-20.3\left(\mathrm{c}=1.0, \mathrm{CHCl}_{3}\right)$. The enantiomeric excess was determined by HPLC on Chiralcel OD-H column, hexane: isopropanol = 98:2; flow rate $=1.0 \mathrm{~mL} / \mathrm{min} ; \mathrm{UV}$ detection at $220 \mathrm{~nm} ; \mathrm{t}_{\mathrm{R}}=10.3 \mathrm{~min}$ (minor), $12.6 \mathrm{~min}$ (major). ${ }^{1} \mathrm{H}$ NMR $\left(400 \mathrm{MHz}, \mathrm{CDCl}_{3}\right) \delta 9.71(\mathrm{~s}, 1 \mathrm{H}), 7.42-7.40(\mathrm{~m}, 2 \mathrm{H}), 7.06-7.04(\mathrm{~m}, 2 \mathrm{H}), 5.01-$ $4.92(\mathrm{~m}, 1 \mathrm{H}), 3.14-3.07$ (m, 1H), 2.97 (dd, $J=13.7,7.0 \mathrm{~Hz}, 1 \mathrm{H}), 2.86-2.79(\mathrm{~m}, 1 \mathrm{H})$, $2.73(\mathrm{dd}, J=13.7,7.9 \mathrm{~Hz}, 1 \mathrm{H}), 2.51(\mathrm{dd}, J=18.2,4.8 \mathrm{~Hz}, 1 \mathrm{H}), 1.21(\mathrm{~d}, J=6.3 \mathrm{~Hz}$, $3 \mathrm{H}), 1.13(\mathrm{~d}, J=6.3 \mathrm{~Hz}, 3 \mathrm{H}) \mathrm{ppm} .{ }^{13} \mathrm{C} \mathrm{NMR}\left(101 \mathrm{MHz}, \mathrm{CDCl}_{3}\right) \delta 199.7,173.2,137.2$, 131.6, 130.7, 120.5, 68.4, 44.5, 40.9, 36.9, 21.6, $21.6 \mathrm{ppm}$. HRMS calculated $[\mathrm{M}+\mathrm{Na}]^{+}$ for $\mathrm{C}_{14} \mathrm{H}_{17} \mathrm{BrNaO}_{3}=335.0253$, found: 335.0248 .<smiles>CCCOC(=O)C(CC=O)Cc1ccccc1OC</smiles>

isopropyl (S)-2-(2-methoxybenzyl)-4-oxobutanoate (2m): $44.9 \mathrm{mg}$, colorless oil; isolated yield: $85 \% ; 96 \%$ ee; $[\alpha]_{\mathrm{D}}^{25}=-22.7\left(\mathrm{c}=1.0, \mathrm{CHCl}_{3}\right)$. The enantiomeric excess was determined by HPLC on Chiralcel OD-H column, hexane: isopropanol = 95:5; flow rate $=1.0 \mathrm{~mL} / \mathrm{min} ; \mathrm{UV}$ detection at $220 \mathrm{~nm} ; \mathrm{t}_{\mathrm{R}}=16.1 \mathrm{~min}$ (minor), $18.5 \mathrm{~min}$ (major). ${ }^{1} \mathrm{H}$ NMR $\left(400 \mathrm{MHz}, \mathrm{CDCl}_{3}\right) \delta 9.68(\mathrm{~s}, 1 \mathrm{H}), 7.23-7.19(\mathrm{~m}, 1 \mathrm{H}), 7.09-7.07(\mathrm{~m}, 1 \mathrm{H}), 6.88-$ 
$6.83(\mathrm{~m}, 2 \mathrm{H}), 5.00-4.91(\mathrm{~m}, 1 \mathrm{H}), 3.82(\mathrm{~s}, 3 \mathrm{H}), 3.25-3.16(\mathrm{~m}, 1 \mathrm{H}), 3.02(\mathrm{dd}, J=13.3$, $6.7 \mathrm{~Hz}, 1 \mathrm{H}), 2.83-2.75(\mathrm{~m}, 2 \mathrm{H}), 2.49(\mathrm{dd}, J=18.1,4.6 \mathrm{~Hz}, 1 \mathrm{H}), 1.21(\mathrm{~d}, J=6.3 \mathrm{~Hz}$, $3 \mathrm{H}), 1.12(\mathrm{~d}, J=6.3 \mathrm{~Hz}, 3 \mathrm{H}) \mathrm{ppm} .{ }^{13} \mathrm{C} \mathrm{NMR}\left(101 \mathrm{MHz}, \mathrm{CDCl}_{3}\right) \delta 200.5,173.9,157.5$, $130.8,128.0,126.4,120.3,110.2,68.0,55.1,44.7,39.5,32.4,21.6,21.5$ ppm. HRMS calculated $[\mathrm{M}+\mathrm{Na}]^{+}$for $\mathrm{C}_{15} \mathrm{H}_{20} \mathrm{NaO}_{4}=287.1254$, found: 287.1254 .<smiles>CCCOC(=O)C(CC=O)Cc1cccc(OC)c1</smiles>

isopropyl (S)-2-(3-methoxybenzyl)-4-oxobutanoate (2n): $42.3 \mathrm{mg}$, colorless oil; isolated yield: $80 \% ; 91 \%$ ee; $[\alpha]_{\mathrm{D}}{ }^{25}=-14.8\left(\mathrm{c}=1.0, \mathrm{CHCl}_{3}\right)$. The enantiomeric excess was determined by HPLC on Chiralcel OD-H column, hexane: isopropanol = 99:1; flow rate $=1.0 \mathrm{~mL} / \mathrm{min}$; $\mathrm{UV}$ detection at $220 \mathrm{~nm} ; \mathrm{t}_{\mathrm{R}}=41.0 \mathrm{~min}$ (major), $45.7 \mathrm{~min}$ (minor). ${ }^{1} \mathrm{H}$ NMR $\left(400 \mathrm{MHz}, \mathrm{CDCl}_{3}\right) \delta 9.70(\mathrm{~s}, 1 \mathrm{H}), 7.20(\mathrm{t}, J=7.9 \mathrm{~Hz}, 1 \mathrm{H}), 6.78-6.71(\mathrm{~m}, 3 \mathrm{H})$, 5.04-4.94 (m, 1H), 3.79 (s, 3H), 3.16-3.09 (m, 1H), $3.03(\mathrm{dd}, J=13.5,6.4 \mathrm{~Hz}, 1 \mathrm{H})$, $2.82(\mathrm{dd}, J=18.2,9.0 \mathrm{~Hz}, 1 \mathrm{H}), 2.72(\mathrm{dd}, J=13.5,8.6 \mathrm{~Hz}, 1 \mathrm{H}), 2.51(\mathrm{dd}, J=18.2,4.5$ $\mathrm{Hz}, 1 \mathrm{H}), 1.23(\mathrm{~d}, J=6.3 \mathrm{~Hz}, 3 \mathrm{H}), 1.16(\mathrm{~d}, J=6.3 \mathrm{~Hz}, 3 \mathrm{H}) \mathrm{ppm} .{ }^{13} \mathrm{C}$ NMR $(101 \mathrm{MHz}$, $\left.\mathrm{CDCl}_{3}\right) \delta 200.1,173.5,159.6,139.7,129.5,121.3,114.7,112.0,68.3,55.1,44.4,41.0$, 37.6, 21.6, $21.6 \mathrm{ppm}$. HRMS calculated $[\mathrm{M}+\mathrm{Na}]^{+}$for $\mathrm{C}_{15} \mathrm{H}_{20} \mathrm{NaO}_{4}=287.1254$, found: 287.1259 .<smiles>CCCOC(=O)C(CC=O)Cc1ccc(OC)cc1</smiles>

isopropyl (S)-2-(4-methoxybenzyl)-4-oxobutanoate (2o): $43.3 \mathrm{mg}$, colorless oil; isolated yield: $82 \% ; 90 \%$ ee; $[\alpha]_{\mathrm{D}}{ }^{25}=-15.2\left(\mathrm{c}=1.0, \mathrm{CHCl}_{3}\right)$. The enantiomeric excess was determined by HPLC on Chiralcel OD-H column, hexane: isopropanol = 98:2; flow rate $=1.0 \mathrm{~mL} / \mathrm{min} ; \mathrm{UV}$ detection at $220 \mathrm{~nm} ; \mathrm{t}_{\mathrm{R}}=12.2 \mathrm{~min}$ (minor), $14.8 \mathrm{~min}$ (major). ${ }^{1} \mathrm{H}$ NMR $\left(400 \mathrm{MHz}, \mathrm{CDCl}_{3}\right) \delta 9.69(\mathrm{~s}, 1 \mathrm{H}), 7.08(\mathrm{~d}, J=8.6 \mathrm{~Hz}, 2 \mathrm{H}), 6.83(\mathrm{~d}, J=8.6$ $\mathrm{Hz}, 2 \mathrm{H}), 5.03-4.93(\mathrm{~m}, 1 \mathrm{H}), 3.78(\mathrm{~s}, 3 \mathrm{H}), 3.12-3.05(\mathrm{~m}, 1 \mathrm{H}), 2.99(\mathrm{dd}, J=13.6,6.5 \mathrm{~Hz}$, $1 \mathrm{H}), 2.84-2.77(\mathrm{~m}, 1 \mathrm{H}), 2.70(\mathrm{dd}, J=13.6,8.4 \mathrm{~Hz}, 1 \mathrm{H}), 2.49$ (dd, $J=18.1,4.5 \mathrm{~Hz}, 1 \mathrm{H})$, $1.22(\mathrm{~d}, J=6.3 \mathrm{~Hz}, 3 \mathrm{H}), 1.15(\mathrm{~d}, J=6.3 \mathrm{~Hz}, 3 \mathrm{H}) \mathrm{ppm} .{ }^{13} \mathrm{C} \mathrm{NMR}\left(101 \mathrm{MHz}, \mathrm{CDCl}_{3}\right) \delta$ 200.2, 173.6, 158.3, 130.1, 130.0, 113.8, 68.2, 55.2, 44.4, 41.3, 36.7, 21.6, 21.6 ppm. HRMS calculated $[\mathrm{M}+\mathrm{Na}]^{+}$for $\mathrm{C}_{15} \mathrm{H}_{20} \mathrm{NaO}_{4}=287.1254$, found: 287.1264 .<smiles>CCOC(=O)[C@H](CC=O)Cc1ccc(C(C)(C)C)cc1</smiles>

ethyl (S)-2-(4-(tert-butyl)benzyl)-4-oxobutanoate (2p): $47.0 \mathrm{mg}$, colorless oil; isolated yield: $85 \% ; 88 \%$ ee; $[\alpha]_{\mathrm{D}}{ }^{25}=-22.3\left(\mathrm{c}=1.0, \mathrm{CHCl}_{3}\right)$. The enantiomeric excess was determined by HPLC on Chiralcel OD-H column, hexane: isopropanol = 95:5; flow 
rate $=1.0 \mathrm{~mL} / \mathrm{min} ; \mathrm{UV}$ detection at $220 \mathrm{~nm} ; \mathrm{t}_{\mathrm{R}}=6.1 \mathrm{~min}$ (minor), $9.2 \mathrm{~min}$ (major). ${ }^{1} \mathrm{H}$ NMR (400 MHz, $\left.\mathrm{CDCl}_{3}\right) \delta 9.70(\mathrm{~s}, 1 \mathrm{H}), 7.32-7.30(\mathrm{~m}, 2 \mathrm{H}), 7.10-7.08(\mathrm{~m}, 2 \mathrm{H}), 4.13(\mathrm{q}$, $J=7.1 \mathrm{~Hz}, 2 \mathrm{H}), 3.18-3.11(\mathrm{~m}, 1 \mathrm{H}), 3.04(\mathrm{dd}, J=13.6,6.2 \mathrm{~Hz}, 1 \mathrm{H}), 2.87-2.79(\mathrm{~m}, 1 \mathrm{H})$, $2.73(\mathrm{dd}, J=13.6,8.7 \mathrm{~Hz}, 1 \mathrm{H}), 2.51(\mathrm{dd}, J=18.2,4.4 \mathrm{~Hz}, 1 \mathrm{H}), 1.30(\mathrm{~s}, 9 \mathrm{H}), 1.20$ (t, $J$ $=7.1 \mathrm{~Hz}, 3 \mathrm{H}) \mathrm{ppm} .{ }^{13} \mathrm{C} \mathrm{NMR}\left(101 \mathrm{MHz}, \mathrm{CDCl}_{3}\right) \delta 200.2,174.2,149.6,134.9,128.6$, $125.4,60.8,44.4,40.9,37.1,34.4,31.3,14.0 \mathrm{ppm}$. HRMS calculated $[\mathrm{M}+\mathrm{Na}]^{+}$for $\mathrm{C}_{17} \mathrm{H}_{24} \mathrm{NaO}_{3}=299.1618$, found: 299.1618 .<smiles>CCOC(=O)C(CC=O)Cc1ccc(C#N)cc1</smiles>

ethyl (S)-2-(4-cyanobenzyl)-4-oxobutanoate (2q): $29.4 \mathrm{mg}$, colorless oil; isolated yield: $60 \% ; 88 \%$ ee; $[\alpha]_{\mathrm{D}}{ }^{25}=-23.5\left(\mathrm{c}=1.0, \mathrm{CHCl}_{3}\right)$. The enantiomeric excess was determined by HPLC on Chiralpak AD-H column, hexane: isopropanol = 90:10; flow rate $=1.0 \mathrm{~mL} / \mathrm{min} ; \mathrm{UV}$ detection at $220 \mathrm{~nm} ; \mathrm{t}_{\mathrm{R}}=17.1 \mathrm{~min}$ (major), $20.3 \mathrm{~min}$ (minor). ${ }^{1} \mathrm{H}$ NMR $\left(400 \mathrm{MHz}, \mathrm{CDCl}_{3}\right) \delta 9.74(\mathrm{~s}, 1 \mathrm{H}), 7.61-7.59(\mathrm{~m}, 2 \mathrm{H}), 7.31-7.29(\mathrm{~m}, 2 \mathrm{H}), 4.13-$ $4.07(\mathrm{~m}, 2 \mathrm{H}), 3.23-3.16(\mathrm{~m}, 1 \mathrm{H}), 3.07(\mathrm{dd}, J=13.6,7.4 \mathrm{~Hz}, 1 \mathrm{H}), 2.91-2.85(\mathrm{~m}, 2 \mathrm{H})$, $2.57(\mathrm{dd}, J=18.4,5.3 \mathrm{~Hz}, 1 \mathrm{H}), 1.18(\mathrm{t}, J=7.1 \mathrm{~Hz}, 3 \mathrm{H}) \mathrm{ppm} .{ }^{13} \mathrm{C}$ NMR (101 MHz, $\left.\mathrm{CDCl}_{3}\right) \delta 199.3,173.4,143.9,132.3,129.8,118.7,110.7,61.1,44.5,40.4,37.5,14.0$ ppm. HRMS calculated $[\mathrm{M}+\mathrm{Na}]^{+}$for $\mathrm{C}_{14} \mathrm{H}_{15} \mathrm{NNaO}_{3}=268.0944$, found: 298.0961 .<smiles>CCOC(=O)[C@H](CC=O)Cc1ccco1</smiles>

ethyl $(\boldsymbol{S})$-2-(furan-2-ylmethyl)-4-oxobutanoate (2r): $34.1 \mathrm{mg}$, colorless oil; isolated yield: $81 \% ; 88 \%$ ee; $[\alpha]_{\mathrm{D}}{ }^{25}=-10.8\left(\mathrm{c}=0.5, \mathrm{CHCl}_{3}\right)$. The enantiomeric excess was determined by HPLC on Chiralcel OD-H column, hexane: isopropanol = 98:2; flow rate $=1.0 \mathrm{~mL} / \mathrm{min} ; \mathrm{UV}$ detection at $220 \mathrm{~nm} ; \mathrm{t}_{\mathrm{R}}=10.9 \mathrm{~min}$ (minor), $14.6 \mathrm{~min}$ (major). ${ }^{1} \mathrm{H} \mathrm{NMR}\left(400 \mathrm{MHz}, \mathrm{CDCl}_{3}\right) \delta 9.71(\mathrm{~s}, 1 \mathrm{H}), 7.32-7.31(\mathrm{~m}, 1 \mathrm{H}), 6.28-6.27(\mathrm{~m}, 1 \mathrm{H}), 6.06$ $(\mathrm{d}, J=3.1 \mathrm{~Hz}, 1 \mathrm{H}), 4.16(\mathrm{q}, J=7.1 \mathrm{~Hz}, 2 \mathrm{H}), 3.28-3.21(\mathrm{~m}, 1 \mathrm{H}), 3.06(\mathrm{dd}, J=15.0,5.8$ $\mathrm{Hz}, 1 \mathrm{H}), 2.93-2.84(\mathrm{~m}, 2 \mathrm{H}), 2.59(\mathrm{dd}, J=18.3,5.0 \mathrm{~Hz}, 1 \mathrm{H}), 1.24(\mathrm{t}, J=7.1 \mathrm{~Hz}, 3 \mathrm{H})$ ppm. ${ }^{13} \mathrm{C}$ NMR $\left(101 \mathrm{MHz}, \mathrm{CDCl}_{3}\right) \delta 199.8,173.5,151.9,141.7,110.2,107.2,61.0$, 44.2, 38.5, 29.7, $14.1 \mathrm{ppm}$. HRMS calculated $[\mathrm{M}+\mathrm{Na}]^{+}$for $\mathrm{C}_{11} \mathrm{H}_{14} \mathrm{NaO}_{4}=233.0784$, found: 233.0781 .<smiles>CCOC(=O)[C@H](CC=O)CCO[Bi]</smiles>

ethyl (S)-4-(benzyloxy)-2-(2-oxoethyl)butanoate (2s): $44.4 \mathrm{mg}$, colorless oil; isolated yield: $84 \% ; 89 \%$ ee; $[\alpha]_{\mathrm{D}}{ }^{25}=-12.8\left(\mathrm{c}=1.0, \mathrm{CHCl}_{3}\right)$. The enantiomeric excess was determined by HPLC on Chiralcel OD-H column, hexane: isopropanol = 98:2; flow 
rate $=1.0 \mathrm{~mL} / \mathrm{min}$; $\mathrm{UV}$ detection at $220 \mathrm{~nm} ; \mathrm{t}_{\mathrm{R}}=16.2 \mathrm{~min}$ (minor), $18.0 \mathrm{~min}$ (major). ${ }^{1} \mathrm{H}$ NMR $\left(400 \mathrm{MHz}, \mathrm{CDCl}_{3}\right) \delta 9.73(\mathrm{~s}, 1 \mathrm{H}), 7.36-7.27(\mathrm{~m}, 5 \mathrm{H}), 4.50-4.44(\mathrm{~m}, 2 \mathrm{H}), 4.14-$ $4.09(\mathrm{~m}, 2 \mathrm{H}), 3.51(\mathrm{t}, J=6.1 \mathrm{~Hz}, 2 \mathrm{H}), 3.11-3.05(\mathrm{~m}, 1 \mathrm{H}), 2.87(\mathrm{dd}, J=18.0,8.6 \mathrm{~Hz}$, $1 \mathrm{H}), 2.61(\mathrm{dd}, J=18.0,5.0 \mathrm{~Hz}, 1 \mathrm{H}), 2.06-1.98(\mathrm{~m}, 1 \mathrm{H}), 1.86-1.78(\mathrm{~m}, 1 \mathrm{H}), 1.23(\mathrm{t}, J=$ $7.1 \mathrm{~Hz}, 3 \mathrm{H}) \mathrm{ppm} .{ }^{13} \mathrm{C} \mathrm{NMR}\left(101 \mathrm{MHz}, \mathrm{CDCl}_{3}\right) \delta 200.1,174.5,138.1,128.3,127.6$, 127.6, 72.9, 67.4, 60.7, 45.1, 36.4, 31.5, $14.1 \mathrm{ppm}$. HRMS calculated $[\mathrm{M}+\mathrm{Na}]^{+}$for $\mathrm{C}_{15} \mathrm{H}_{20} \mathrm{NaO}_{4}=287.1254$, found: 287.1251.<smiles>CCCCC[C@H](CC=O)C(=O)OCC</smiles>

ethyl (S)-2-(2-oxoethyl)heptanoate (2t): $33.2 \mathrm{mg}$, colorless oil; isolated yield: 83\%; $88 \%$ ee; $[\alpha]_{\mathrm{D}}{ }^{25}=-9.9\left(\mathrm{c}=1.0, \mathrm{CHCl}_{3}\right)$. The enantiomeric excess was determined by HPLC on Chiralcel OD-H column, hexane: isopropanol $=98: 2$; flow rate $=1.0 \mathrm{~mL} / \mathrm{min}$; $\mathrm{UV}$ detection at $220 \mathrm{~nm} ; \mathrm{t}_{\mathrm{R}}=5.3 \mathrm{~min}$ (minor), $6.0 \mathrm{~min}$ (major). ${ }^{1} \mathrm{H} \mathrm{NMR}(400 \mathrm{MHz}$, $\left.\mathrm{CDCl}_{3}\right) \delta 9.77(\mathrm{~s}, 1 \mathrm{H}), 4.18-4.13(\mathrm{~m}, 2 \mathrm{H}), 2.92-2.84(\mathrm{~m}, 2 \mathrm{H}), 2.59-2.51(\mathrm{~m}, 1 \mathrm{H}), 1.68-$ $1.61(\mathrm{~m}, 1 \mathrm{H}), 1.54-1.47(\mathrm{~m}, 1 \mathrm{H}), 1.29-1.25(\mathrm{~m}, 9 \mathrm{H}), 0.88(\mathrm{t}, J=6.8 \mathrm{~Hz}, 3 \mathrm{H}) \mathrm{ppm} .{ }^{13} \mathrm{C}$ NMR $\left(101 \mathrm{MHz}, \mathrm{CDCl}_{3}\right) \delta 200.5,174.9,60.7,45.4,39.0,31.9,31.5,26.6,22.4,14.2$, 14.0 ppm. HRMS calculated $[\mathrm{M}+\mathrm{Na}]^{+}$for $\mathrm{C}_{11} \mathrm{H}_{20} \mathrm{NaO}_{3}=223.1305$, found: 223.1305 .<smiles>COC(=O)C(C)CC=O</smiles>

methyl $(\boldsymbol{S})$-2-methyl-4-oxobutanoate $(\mathbf{2 u}):^{1} 23.9 \mathrm{mg}$, colorless oil; isolated yield: $92 \% ; 93 \%$ ee; $[\alpha]_{\mathrm{D}}{ }^{25}=-8.1\left(\mathrm{c}=1.0, \mathrm{CHCl}_{3}\right)$. The enantiomeric excess was determined by HPLC on Chiralcel OD-H column, hexane: isopropanol $=98: 2$; flow rate $=1.0$ $\mathrm{mL} / \mathrm{min}$; UV detection at $220 \mathrm{~nm} ; \mathrm{t}_{\mathrm{R}}=8.4 \mathrm{~min}$ (minor), $11.5 \mathrm{~min}$ (major). ${ }^{1} \mathrm{H} \mathrm{NMR}$ $\left(400 \mathrm{MHz}, \mathrm{CDCl}_{3}\right) \delta 9.77(\mathrm{~s}, 1 \mathrm{H}), 3.70(\mathrm{~s}, 3 \mathrm{H}), 3.04-2.97(\mathrm{~m}, 1 \mathrm{H}), 2.92(\mathrm{dd}, J=17.8$, $7.9 \mathrm{~Hz}, 1 \mathrm{H}), 2.55(\mathrm{dd}, J=17.8,5.3 \mathrm{~Hz}, 1 \mathrm{H}), 1.24(\mathrm{~d}, J=7.0 \mathrm{~Hz}, 3 \mathrm{H}) \mathrm{ppm} .{ }^{13} \mathrm{C} \mathrm{NMR}$ $\left(101 \mathrm{MHz}, \mathrm{CDCl}_{3}\right) \delta 200.1,175.6,52.0,46.9,33.5,17.0 \mathrm{ppm}$.<smiles>COC(=O)CC(CC=O)C(=O)OC</smiles>

dimethyl (R)-2-(2-oxoethyl)succinate (2v): $34.3 \mathrm{mg}$, light yellow oil; isolated yield: $91 \% ; 86 \%$ ee; $[\alpha]_{\mathrm{D}}{ }^{25}=-0.4\left(\mathrm{c}=1.0, \mathrm{CHCl}_{3}\right)$. The enantiomeric excess was determined by HPLC on Chiralpak AD-H column, hexane: isopropanol $=99: 1$; flow rate $=0.5$ $\mathrm{mL} / \mathrm{min}$; $\mathrm{UV}$ detection at $220 \mathrm{~nm}$; $\mathrm{t}_{\mathrm{R}}=42.4 \mathrm{~min}$ (major), $43.9 \mathrm{~min}$ (minor). ${ }^{1} \mathrm{H} \mathrm{NMR}$ $\left(400 \mathrm{MHz}, \mathrm{CDCl}_{3}\right) \delta 9.77(\mathrm{~s}, 1 \mathrm{H}), 3.71(\mathrm{~d}, J=7.5 \mathrm{~Hz}, 6 \mathrm{H}), 3.38-3.31(\mathrm{~m}, 1 \mathrm{H}), 2.98(\mathrm{dd}$, $J=18.4,6.8 \mathrm{~Hz}, 1 \mathrm{H}), 2.80-2.72(\mathrm{~m}, 2 \mathrm{H}), 2.61(\mathrm{dd}, J=16.8,6.6 \mathrm{~Hz}, 1 \mathrm{H}) \mathrm{ppm} .{ }^{13} \mathrm{C} \mathrm{NMR}$ $\left(101 \mathrm{MHz} \mathrm{CDCl}_{3}\right) \delta 199.4,173.6,171.8,52.4,52.0,44.4,35.1,34.9$ ppm. HRMS 
calculated $[\mathrm{M}+\mathrm{Na}]^{+}$for $\mathrm{C}_{8} \mathrm{H}_{12} \mathrm{NaO}_{5}=211.0577$, found: 211.0575 .<smiles>CCOC(=O)C(CC=O)C(C)C</smiles>

ethyl (R)-2-isopropyl-4-oxobutanoate $(\mathbf{2 w}):{ }^{1} 29.6 \mathrm{mg}$, colorless oil; isolated yield: $86 \% ; 73 \%$ ee; $[\alpha]_{\mathrm{D}}^{25}=-8.4\left(\mathrm{c}=0.25, \mathrm{CHCl}_{3}\right)$. The enantiomeric excess was determined by HPLC on Chiralcel OD-H column, hexane: isopropanol $=98: 2$; flow rate $=1.0$ $\mathrm{mL} / \mathrm{min}$; $\mathrm{UV}$ detection at $220 \mathrm{~nm}$; $\mathrm{t}_{\mathrm{R}}=5.8 \mathrm{~min}$ (minor), $11.4 \mathrm{~min}$ (major). ${ }^{1} \mathrm{H} \mathrm{NMR}$ $\left(400 \mathrm{MHz}, \mathrm{CDCl}_{3}\right) \delta 9.80(\mathrm{~s}, 1 \mathrm{H}), 4.20-4.12(\mathrm{~m}, 2 \mathrm{H}), 2.92(\mathrm{dd}, J=17.9,10.4 \mathrm{~Hz}, 1 \mathrm{H})$, 2.80- $2.75(\mathrm{~m}, 1 \mathrm{H}), 2.51(\mathrm{dd}, J=17.9,3.4 \mathrm{~Hz}, 1 \mathrm{H}), 2.08-2.00(\mathrm{~m}, 1 \mathrm{H}), 1.27(\mathrm{t}, J=7.1$ $\mathrm{Hz}, 3 \mathrm{H}), 0.94(\mathrm{dd}, J=9.0,7.0 \mathrm{~Hz}, 6 \mathrm{H}) \mathrm{ppm} .{ }^{13} \mathrm{C} \mathrm{NMR}\left(101 \mathrm{MHz}, \mathrm{CDCl}_{3}\right) \delta 200.8$, $174.2,60.5,45.1,42.3,29.8,20.1,19.4,14.2 \mathrm{ppm}$.

\section{Procedures for gram-scale AHF of $1 \mathrm{u}$ and the synthesis of $4 \mathrm{a}$}

A stock solution was made by mixing $(S, S)$-DTBM-YanPhos $(0.01 \mathrm{mmol})$ with $\mathrm{Rh}(\mathrm{acac})(\mathrm{CO})_{2}(0.005 \mathrm{mmol})$ in toluene $(2 \mathrm{~mL})$ at room temperature for $10 \mathrm{~min}$ in a argon-filled glovebox. Then, the catalyst solution was transferred by syringe into a 5 $\mathrm{mL}$ vial charged with substrate $\mathbf{1 u}(10 \mathrm{mmol}, 1.0 \mathrm{~g})$. The vial was transferred into an autoclave and taken out of the glovebox. The argon gas was replacement with hydrogen gas for three times, and then hydrogen ( 5 bar) and carbon monoxide ( 5 bar) were charged in sequence. The reaction mixture was stirred at $80{ }^{\circ} \mathrm{C}$ (oil bath) for $48 \mathrm{~h}$. The reaction was cooled to room temperature and the pressure was carefully released in a well-ventilated hood. The solution was concentrated and $\mathbf{2} \mathbf{u}$ was isolated by column chromatography. Isolated yield: $91 \%, 1.2 \mathrm{~g}$.

4a was prepared according to the literature. ${ }^{3}$ To a solution of the chiral aldehyde $\mathbf{2 u}(9.1$ mmol, $1.2 \mathrm{~g}$ ) with $\mathrm{CH}_{2} \mathrm{Cl}_{2}(5 \mathrm{~mL})$ in a $25 \mathrm{ml}$ flask, methyl 2-(triphenyl- $\lambda^{4}$ phosphanyl)acetate $(10 \mathrm{mmol}, 3.3 \mathrm{~g})$ was added, the mixture was allowed to stirred at $25{ }^{\circ} \mathrm{C}$ for 2 hours. The organic solvents were removed under reduced pressure and the residue was purified by flash chromatography to give the desired product $\mathbf{4 a}(1.5 \mathrm{~g}, 90 \%$ yield) with $93 \%$ ee. ${ }^{1} \mathrm{H}$ NMR $\left(400 \mathrm{MHz}, \mathrm{CDCl}_{3}\right) \delta 6.92-6.85(\mathrm{~m}, 1 \mathrm{H}), 5.87(\mathrm{~d}, J=15.7$ $\mathrm{Hz}, 1 \mathrm{H}), 3.73$ (s, 3H), 3.69 (s, 3H), 2.65-2.54 (m, 2H), 2.37-2.29 (m, 1H), 1.19 (d, J= $6.9 \mathrm{~Hz}, 3 \mathrm{H}) \mathrm{ppm} .{ }^{13} \mathrm{C} \mathrm{NMR}\left(101 \mathrm{MHz}, \mathrm{CDCl}_{3}\right) \delta 175.8,145.8,122.9,51.8,51.5,38.4$, $35.8,29.7,16.8 \mathrm{ppm}$.

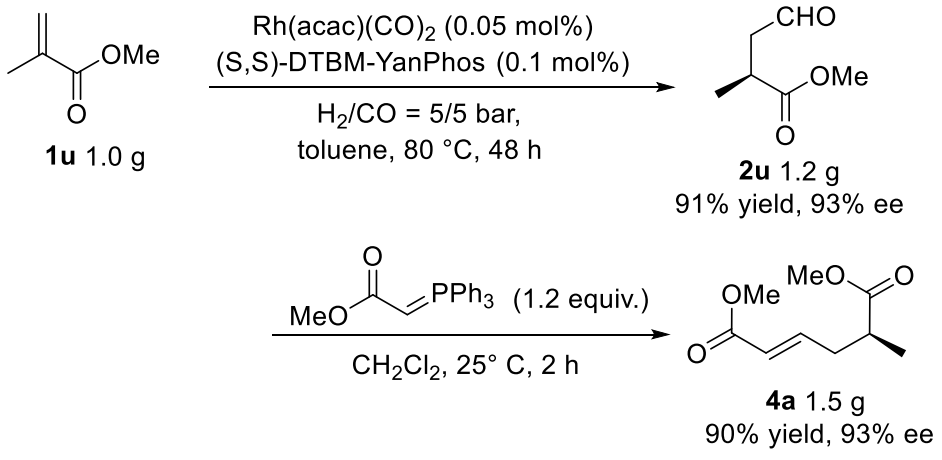




\section{Procedures for gram-scale AHF of $1 c$ and the synthesis of $\mathbf{4 b}$}

A stock solution was made by mixing $(S, S)$-DTBM-YanPhos $(0.01 \mathrm{mmol})$ with $\mathrm{Rh}(\mathrm{acac})(\mathrm{CO})_{2}(0.005 \mathrm{mmol})$ in toluene $(2 \mathrm{~mL})$ at room temperature for $10 \mathrm{~min}$ in a argon-filled glovebox. Then, the catalyst solution was transferred by syringe into a 5 $\mathrm{mL}$ vial charged with substrate $1 \mathrm{c}(5 \mathrm{mmol}, 1.0 \mathrm{~g})$. The vial was transferred into an autoclave and taken out of the glovebox. The argon gas was replacement with hydrogen gas for three times, and then hydrogen ( 5 bar) and carbon monoxide ( 5 bar) were charged in sequence. The reaction mixture was stirred at $80{ }^{\circ} \mathrm{C}$ (oil bath) for $48 \mathrm{~h}$. The reaction was cooled to room temperature and the pressure was carefully released in a well-ventilated hood. The solution was concentrated and $\mathbf{2 c}$ was isolated by column chromatography. Isolated yield: $89 \%, 1.0 \mathrm{~g}$.

4b was prepared according to the literature. ${ }^{4}$ To a solution of $\mathrm{NaBH}_{4}(9 \mathrm{mmol}, 0.34 \mathrm{~g})$ with $5 \mathrm{~mL} \mathrm{CH}_{3} \mathrm{OH}$ in a $25 \mathrm{~mL}$ flask, $2 \mathbf{c}$ was added dropwise at $0{ }^{\circ} \mathrm{C}$, and the mixture was stirred for 15 minutes at $0{ }^{\circ} \mathrm{C}$. Water was added dropwise to quench the reaction, and the mixture was concentrated to remove methanol and then was extracted with ethyl acetate $(3 \times 5 \mathrm{ml})$, dried over anhydrous $\mathrm{MgSO}_{4}$ followed by evaporation. The crude product was dissolved in $5 \mathrm{~mL}$ trifluoroacetic acid and $2.5 \mathrm{~mL}$ water. The mixture was stirred at room temperature for 4 hours. Saturated sodium bicarbonate solution was added dropwise to consume the excess trifluoroacetic acid, then the mixture was extracted with ethyl acetate $(3 \times 10 \mathrm{ml})$, and the combined extracts were washed with brine, and then dried over anhydrous $\mathrm{MgSO}_{4}$ followed by evaporation. The residue was purified by a silica-gel column chromatography (petroleum ether / ethyl acetate, 10:1 by volume) to yield compound $\mathbf{4 b}$ in the form of a colorless oil. Two steps yield: $86 \%$, $91 \%$ ee. $[\alpha]_{\mathrm{D}}^{25}=+52.5\left(\mathrm{c}=0.25, \mathrm{CHCl}_{3}\right)$. The enantiomeric excess was determined by HPLC on Chiralcel OD-H column, hexane: isopropanol $=98: 2$; flow rate $=1.0 \mathrm{~mL} / \mathrm{min}$; UV detection at $220 \mathrm{~nm} ; \mathrm{t}_{\mathrm{R}}=29.7 \mathrm{~min}$ (major), $33.8 \mathrm{~min}$ (minor). ${ }^{1} \mathrm{H} \mathrm{NMR}(400 \mathrm{MHz}$, $\left.\mathrm{CDCl}_{3}\right) \delta 7.33-7.20(\mathrm{~m}, 5 \mathrm{H}), 4.26-4.21(\mathrm{~m}, 1 \mathrm{H}), 4.18-4.12(\mathrm{~m}, 1 \mathrm{H}), 3.26(\mathrm{dd}, J=13.6$, $4.0 \mathrm{~Hz}, 1 \mathrm{H}), 2.89-2.81(\mathrm{~m}, 1 \mathrm{H}), 2.75(\mathrm{dd}, J=13.6,9.5 \mathrm{~Hz}, 1 \mathrm{H}), 2.29-2.21(\mathrm{~m}, 1 \mathrm{H})$, 2.05-1.94 (m, 1H) ppm. ${ }^{13} \mathrm{C}$ NMR (101 MHz, $\left.\mathrm{CDCl}_{3}\right) \delta 178.7,138.4,128.8,128.7$, 126.7, 66.5, 41.1, 36.1, 28.0 ppm.

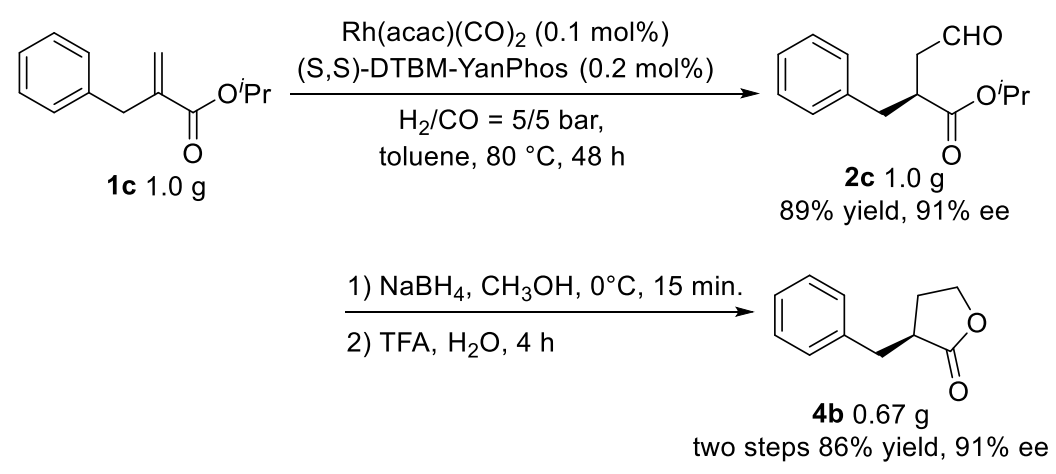




\section{References}

(1) Wang, X.; Buchwald, S. L. Rh-Catalyzed Asymmetric Hydroformyla-tion of Functionalized 1,1-Disubstituted Olefins. J. Am. Chem. Soc. 2011, 133, 19080.

(2) Zhang, C.; Maeda, Y.; Sato, Y. Chem. Pharm. Bull. 1998, 46, 572.

(3) Breit, B.; Zahn, S. K. Domino Hydroformylation-Wittig Reactions. Angew. Chem. Int. Ed. 1999, 38, 969.

(4) Sadhukhan, S.; Zhang, G-F.; Tochtrop, G. P. Modular Isotopomer Synthesis of $\gamma$ Hydroxybutyric Acid for a Quantitative Analysis of Metabolic Fates. ACS Chem. Biol. 2014, 9, 1706. 
7. NMR spectra<smiles>CCOC(=O)C(CC=O)Cc1ccccc1</smiles>

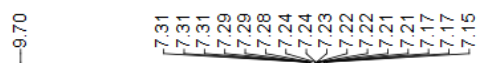

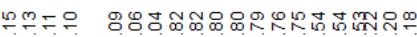

年<smiles>CCOC(=O)C(CC=O)Cc1ccccc1</smiles>
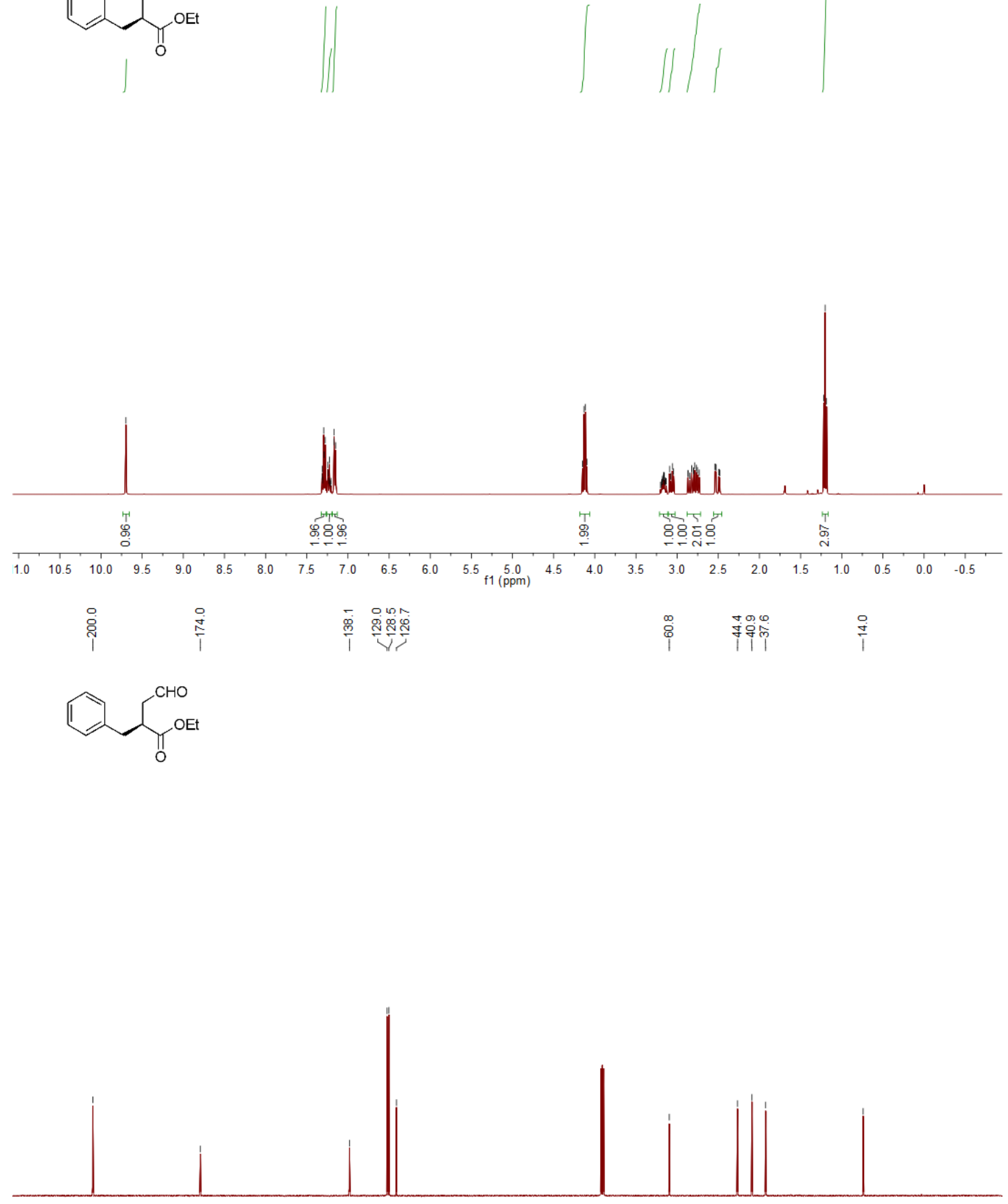

$\begin{array}{llllllllllllllllllllllllll}210 & 200 & 190 & 180 & 170 & 160 & 150 & 140 & 130 & 120 & 110 & 100 & 90 & 80 & 70 & 60 & 50 & 40 & 30 & 20 & 10 & 0 & -10\end{array}$ 
<smiles>COC(=O)C(CC=O)Cc1ccccc1</smiles>

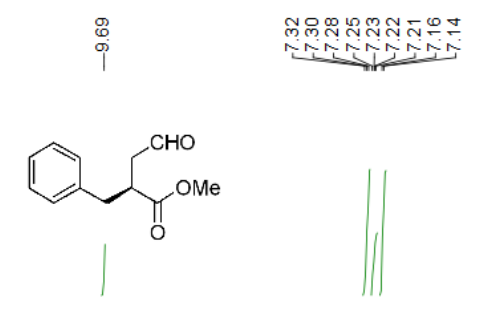

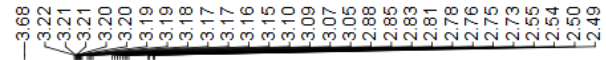

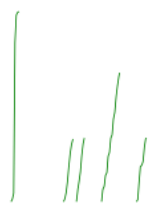

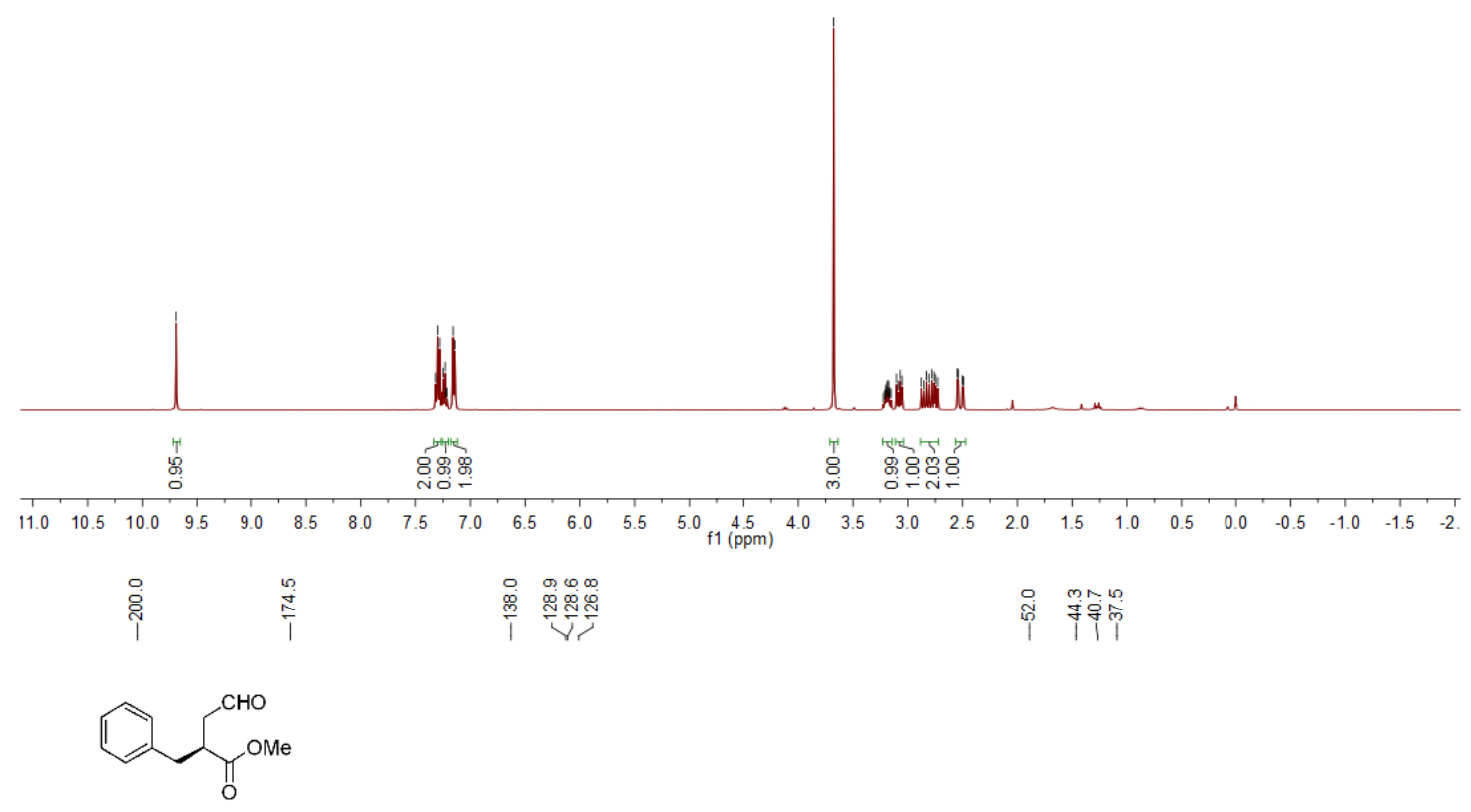

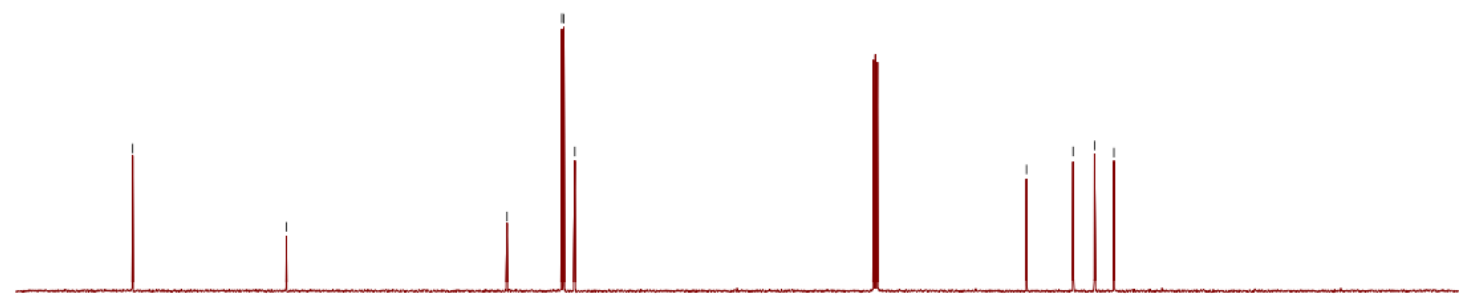

$\begin{array}{llllllllllllllllllllllllllllll}210 & 200 & 190 & 180 & 170 & 160 & 150 & 140 & 130 & 120 & 110 & 100 & 90 & 80 & 70 & 60 & 50 & 40 & 30 & 20 & 10 & 0 & -10\end{array}$ 


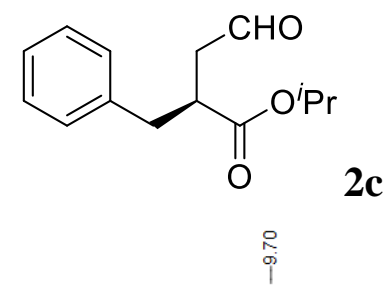

$\underbrace{\mathrm{CHO}}_{0}$

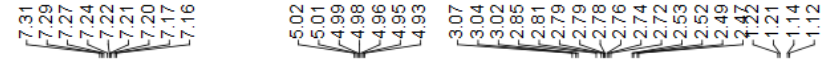

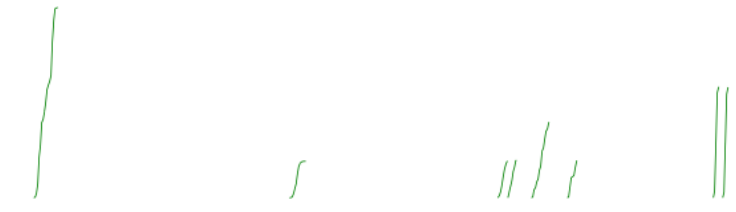

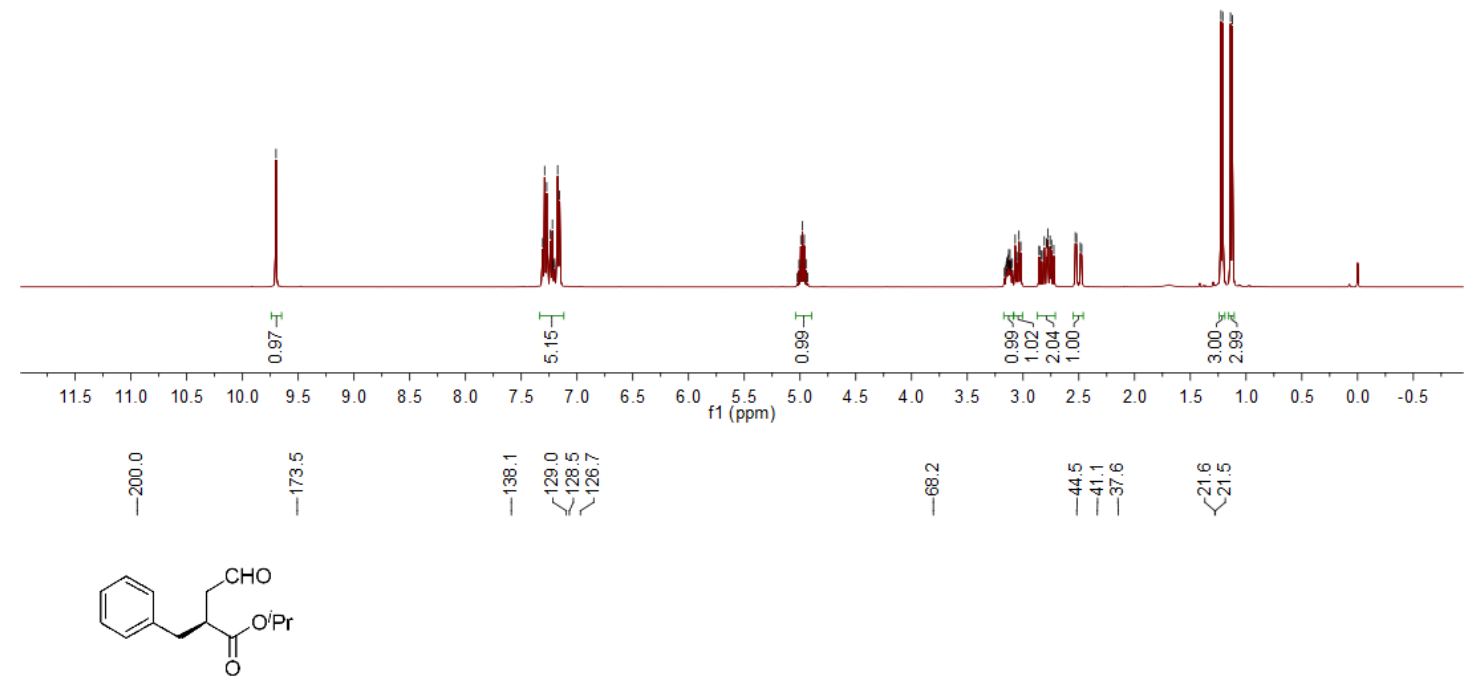

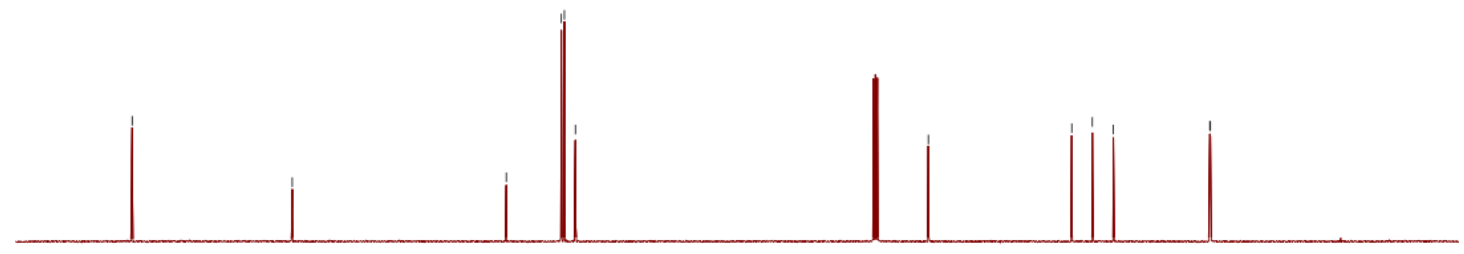

$\begin{array}{lllllllllllllllllllllllllllll}210 & 200 & 190 & 180 & 170 & 160 & 150 & 140 & 130 & 120 & 110 & 100 & 90 & 80 & 70 & 60 & 50 & 40 & 30 & 20 & 10 & 0 & -10\end{array}$ 
<smiles>O=CCC(Cc1ccccc1)C(=O)OC(c1ccccc1)c1ccccc1</smiles>

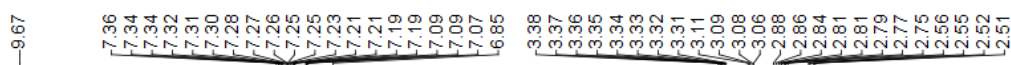

10 $\int_{0}^{\mathrm{CHO}} Y_{\mathrm{Ph}}^{\mathrm{Ph}}$
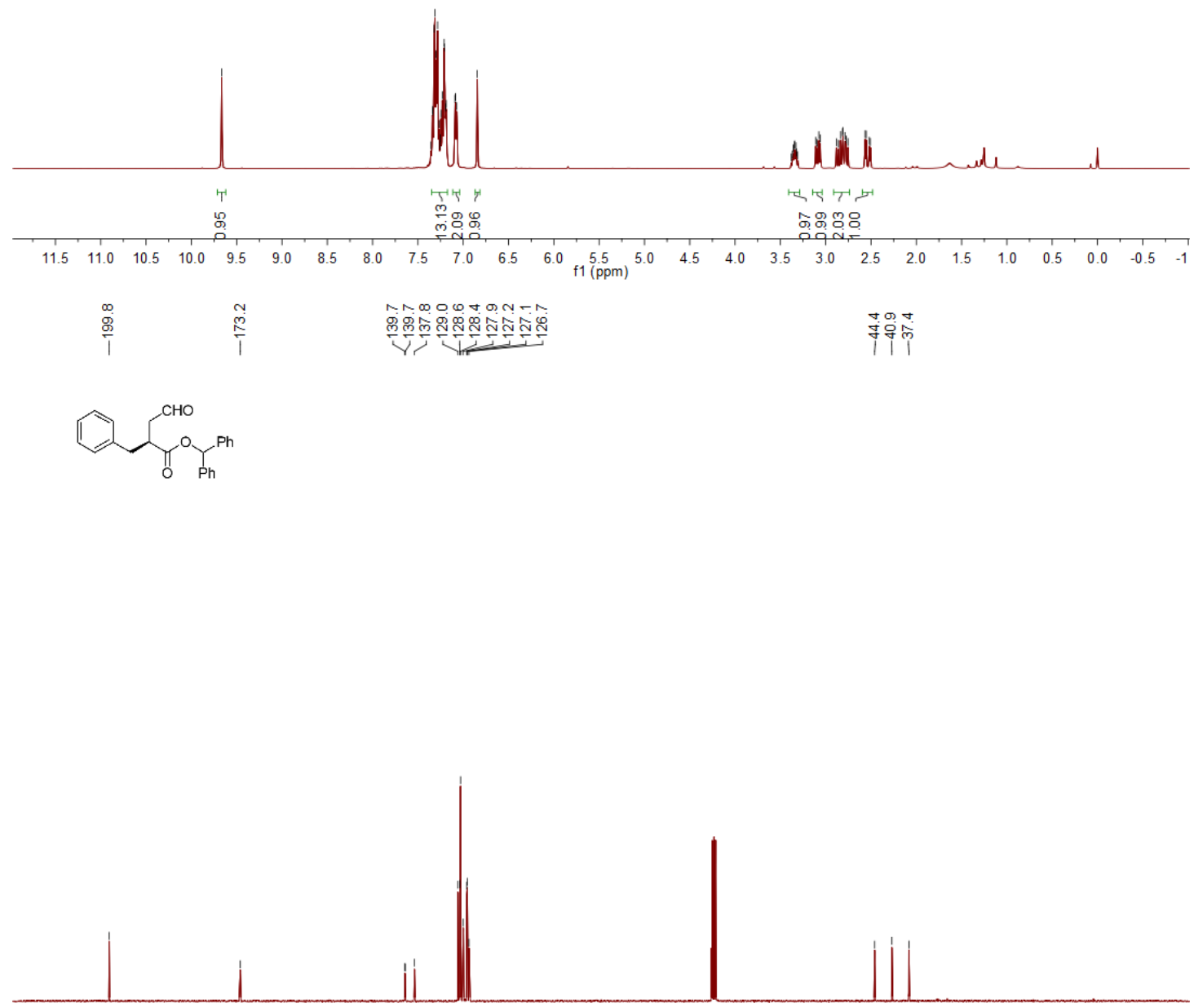

$\begin{array}{lllllllllllllllllllllllllllllll}210 & 200 & 190 & 180 & 170 & 160 & 150 & 140 & 130 & 120 & 110 & 100 & 90 & 80 & 70 & 60 & 50 & 40 & 30 & 20 & 10 & 0 & -10\end{array}$ 
<smiles>O=CCC(Cc1ccccc1)C(=O)Oc1ccccc1</smiles>

每<smiles>O=C(O)C(CCc1ccccc1)Cc1ccccc1</smiles>
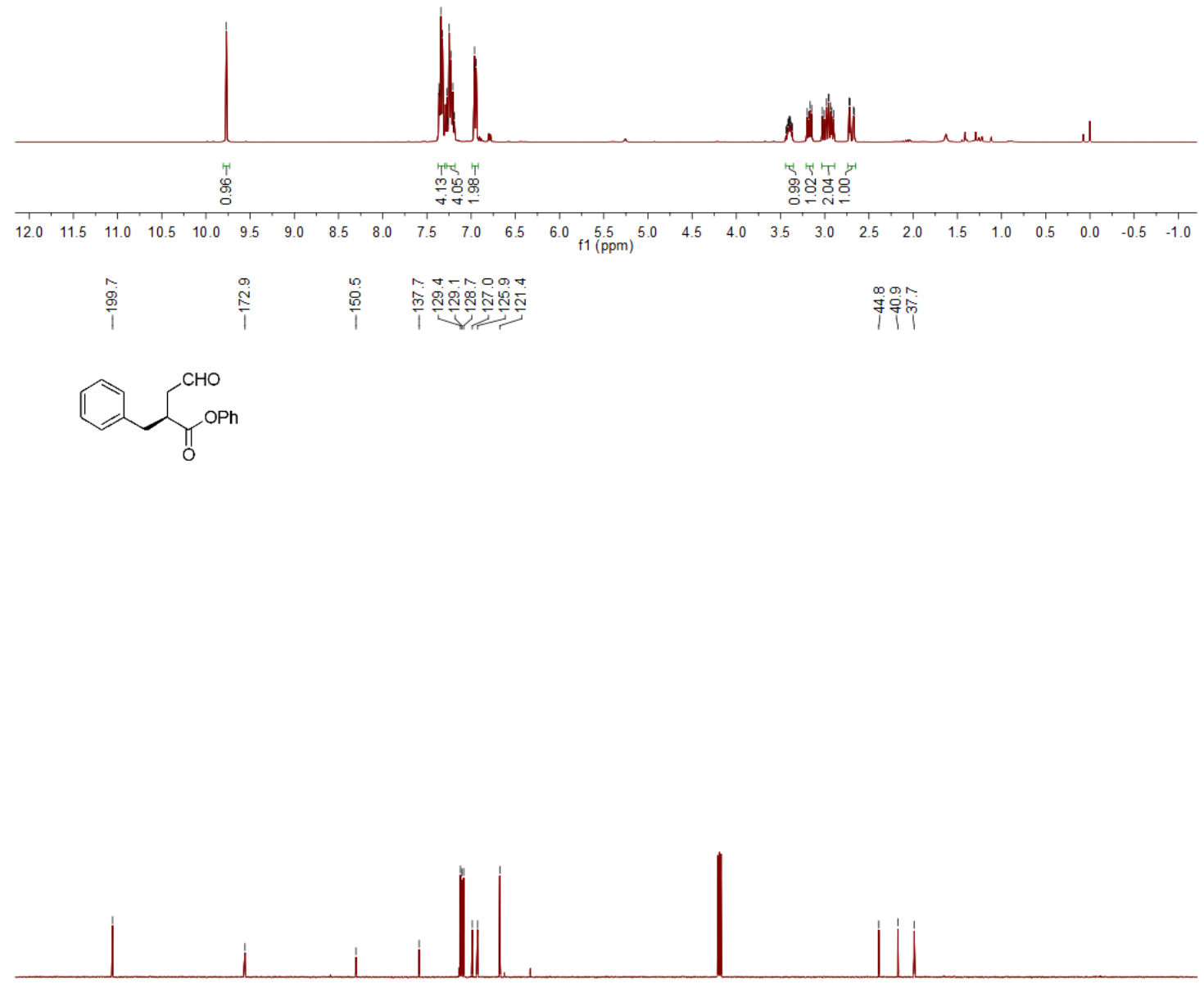

$\begin{array}{llllllllllllllllllllllllllllll}210 & 200 & 190 & 180 & 170 & 160 & 150 & 140 & 130 & 120 & 110 & 100 & 90 & 80 & 70 & 60 & 50 & 40 & 30 & 20 & 10 & 0 & -10\end{array}$ 
<smiles>CCCOC(=O)C(CC=O)Cc1ccccc1</smiles>

$\underset{\substack{0 \\ i}}{\infty}$<smiles>O=C(O)C(CCc1ccccc1)Cc1ccccc1</smiles>

s mpong

ए人

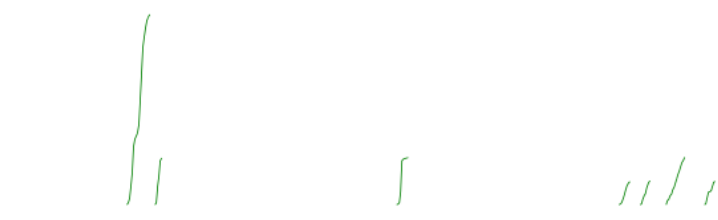

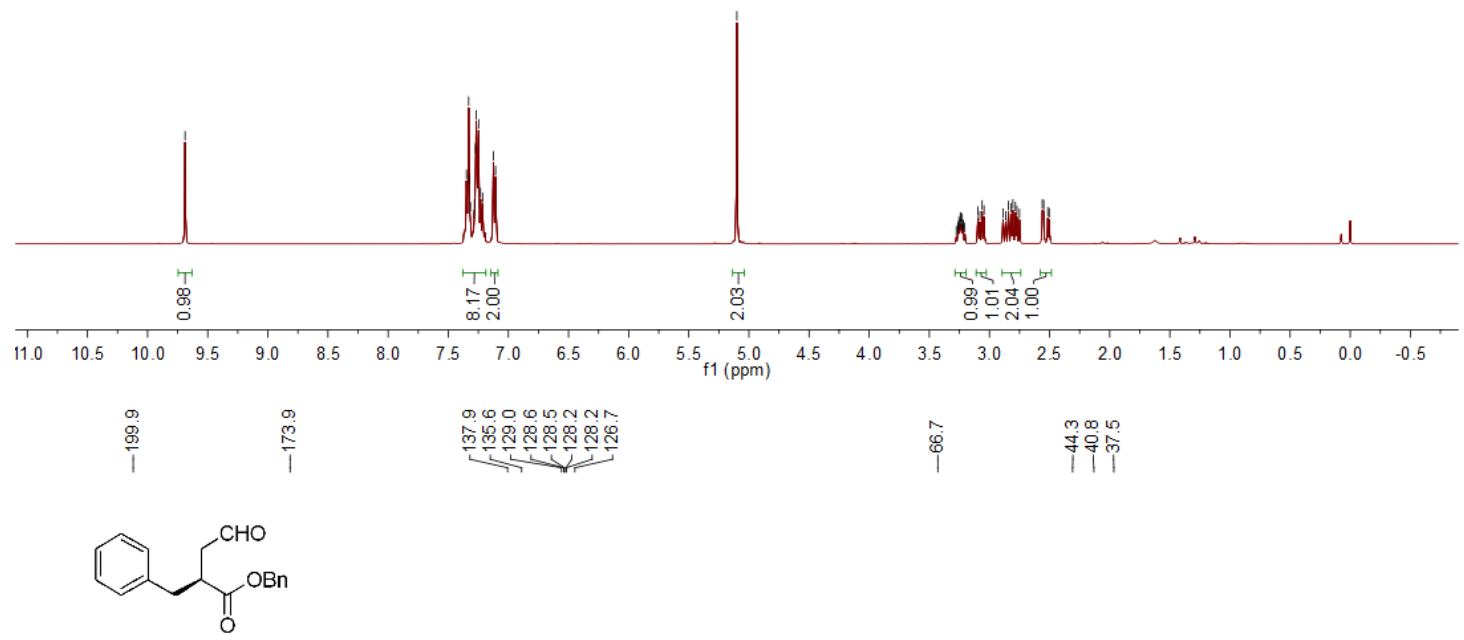

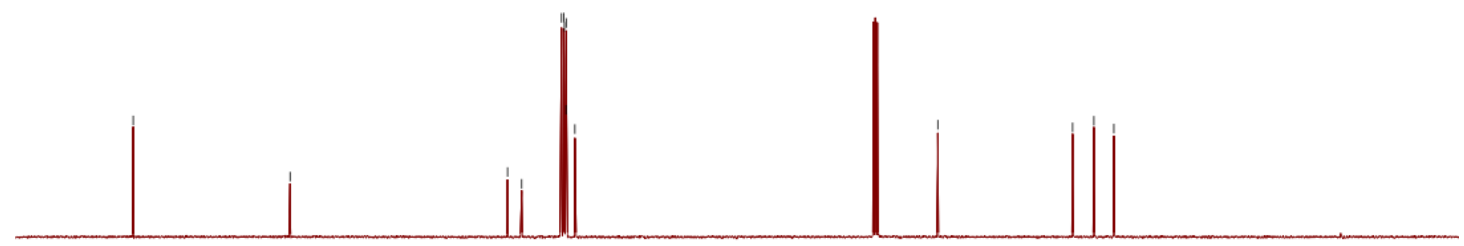

$\begin{array}{llllllllllllllllllllllllllllll}210 & 200 & 190 & 180 & 170 & 160 & 150 & 140 & 130 & 120 & 110 & 100 & 90 & 80 & 70 & 60 & 50 & 40 & 30 & 20 & 10 & 0 & -10\end{array}$ 


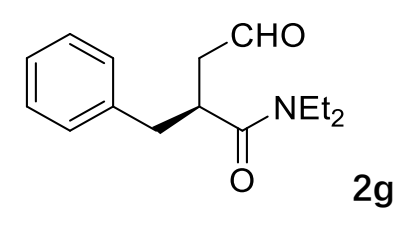

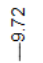

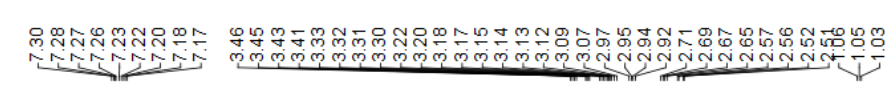
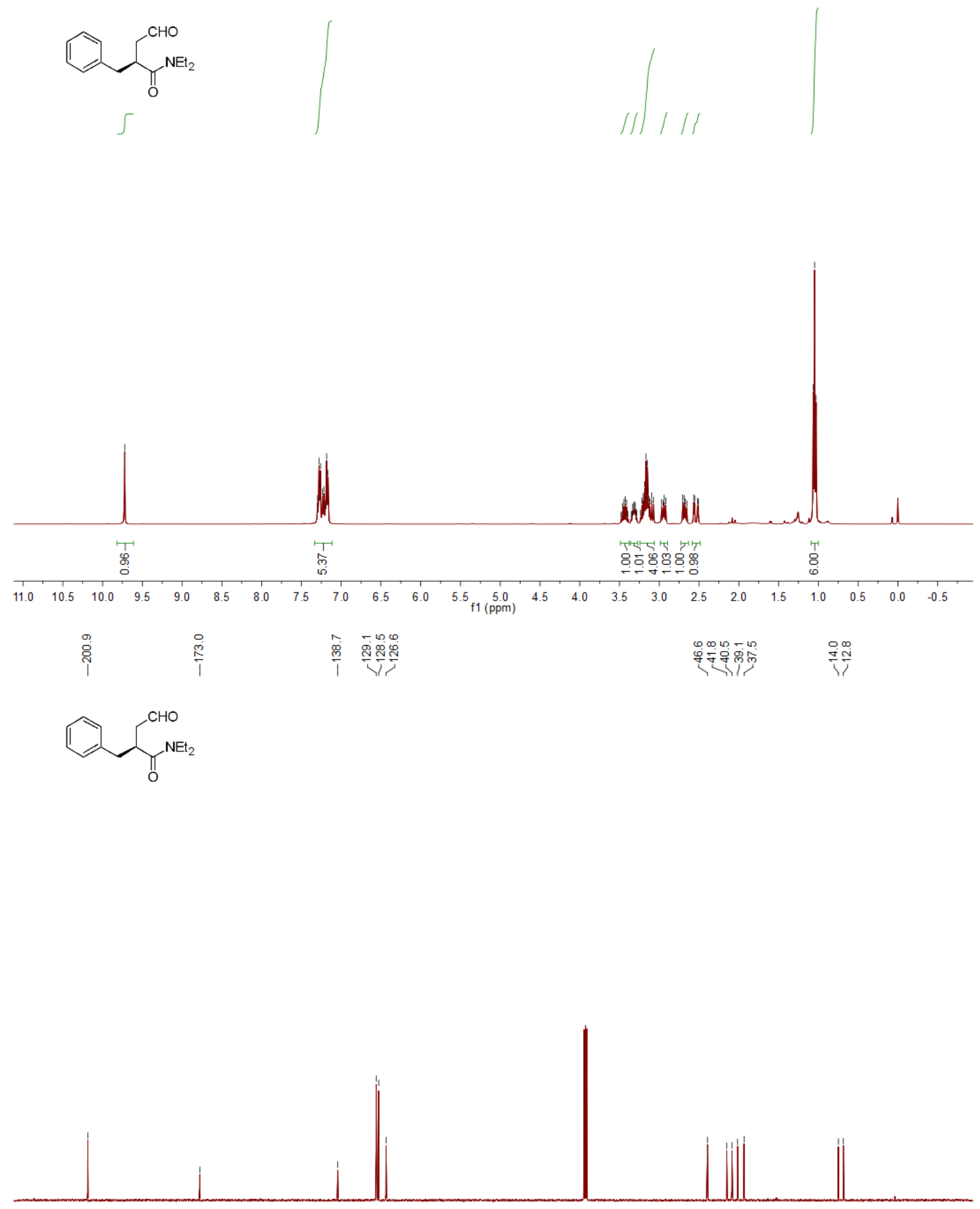

$\begin{array}{lllllllllllllllllllllllllllllll}210 & 200 & 190 & 180 & 170 & 160 & 150 & 140 & 130 & 120 & 110 & 100 & 90 & 80 & 70 & 60 & 50 & 40 & 30 & 20 & 10 & 0 & -10\end{array}$ 
<smiles>CCC(Cc1ccccc1)C(=O)N1CCCCO1</smiles>

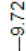

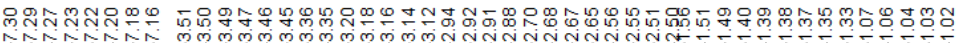<smiles>CCC(Cc1ccccc1)C(=O)N1CCCCO1</smiles>

ऽ
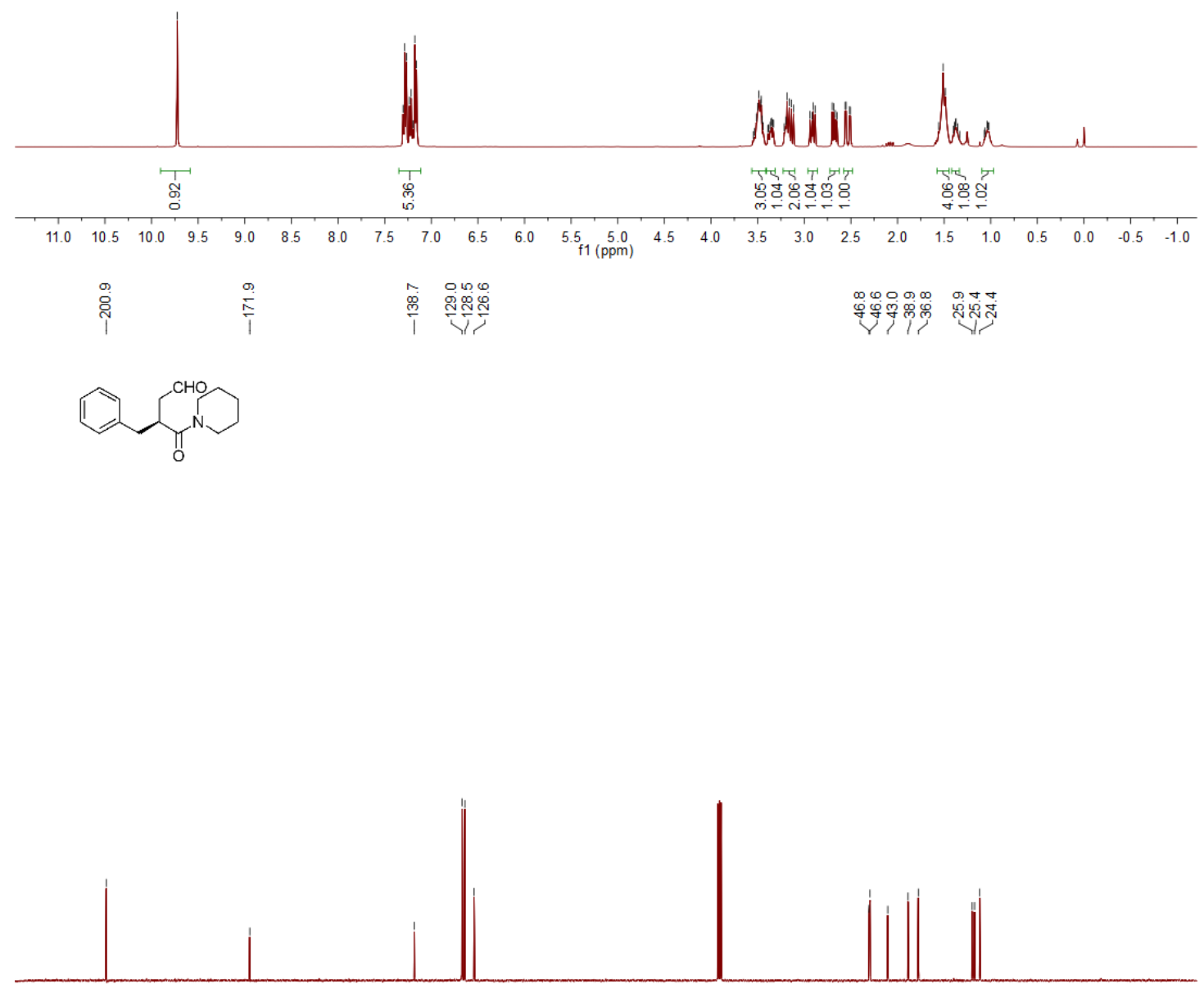

$\begin{array}{llllllllllllllllllllllllllll}210 & 200 & 190 & 180 & 170 & 160 & 150 & 140 & 130 & 120 & 110 & 100 & 90 & 80 & 70 & 60 & 50 & 40 & 30 & 20 & 10 & 0 & -10\end{array}$ 

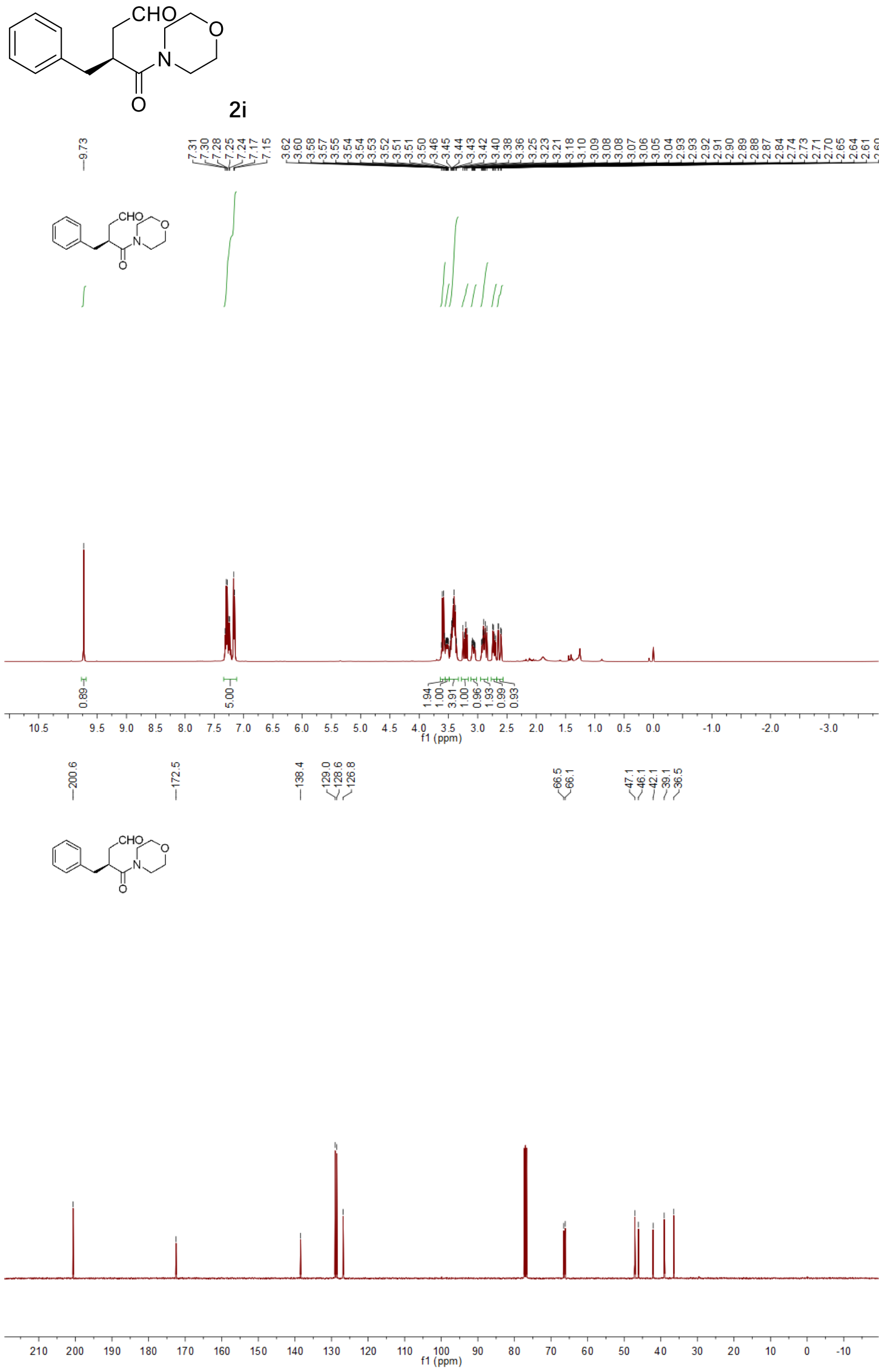

S22 
<smiles>O=CCC(Cc1ccc(F)cc1)C(=O)O[Te]</smiles>
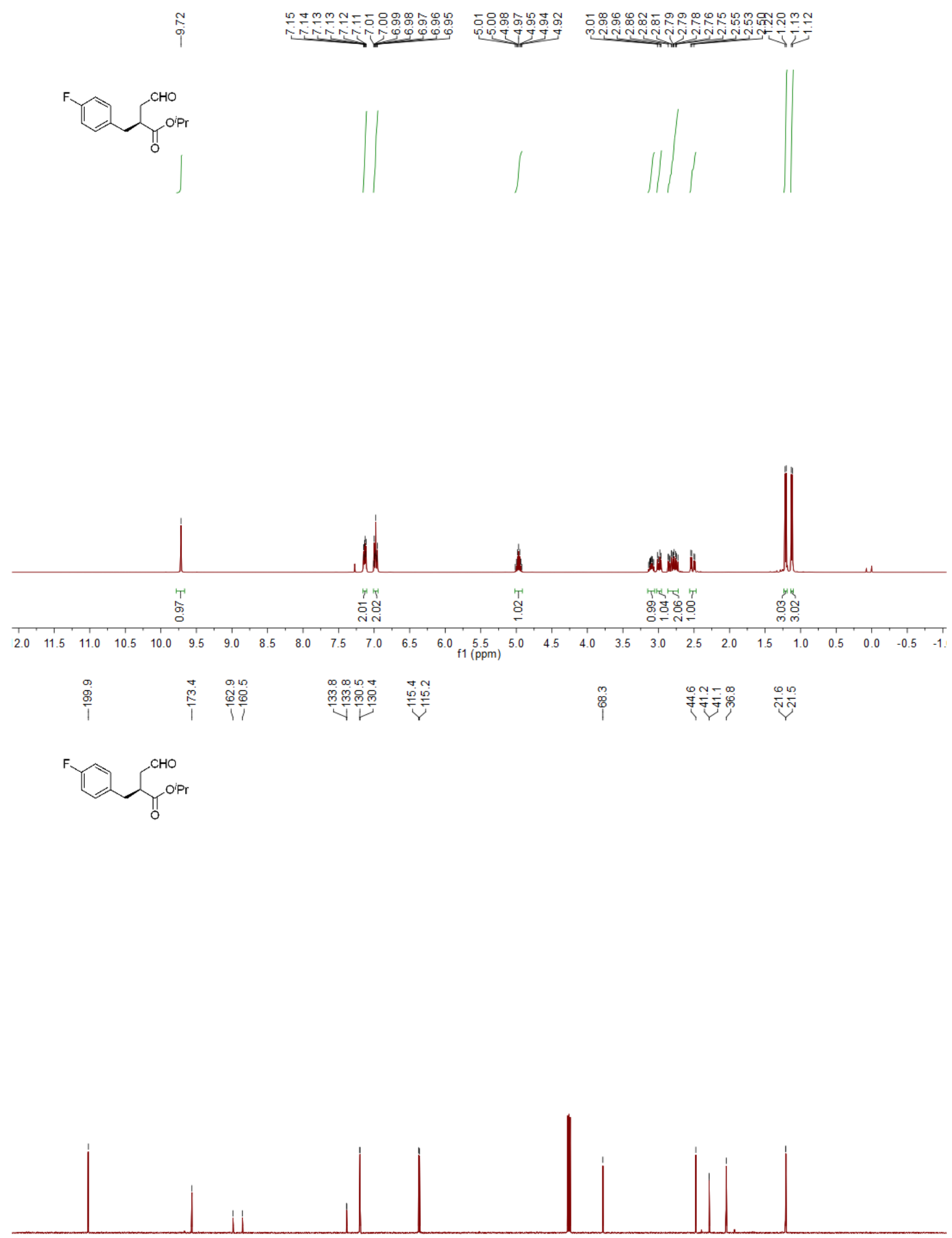

$\begin{array}{llllllllllllllllllllllllllllll}210 & 200 & 190 & 180 & 170 & 160 & 150 & 140 & 130 & 120 & 110 & 100 & 90 & 80 & 70 & 60 & 50 & 40 & 30 & 20 & 10 & 0 & -10\end{array}$ 
<smiles>CCCOC(=O)C(CC=O)Cc1ccc(Cl)cc1</smiles>
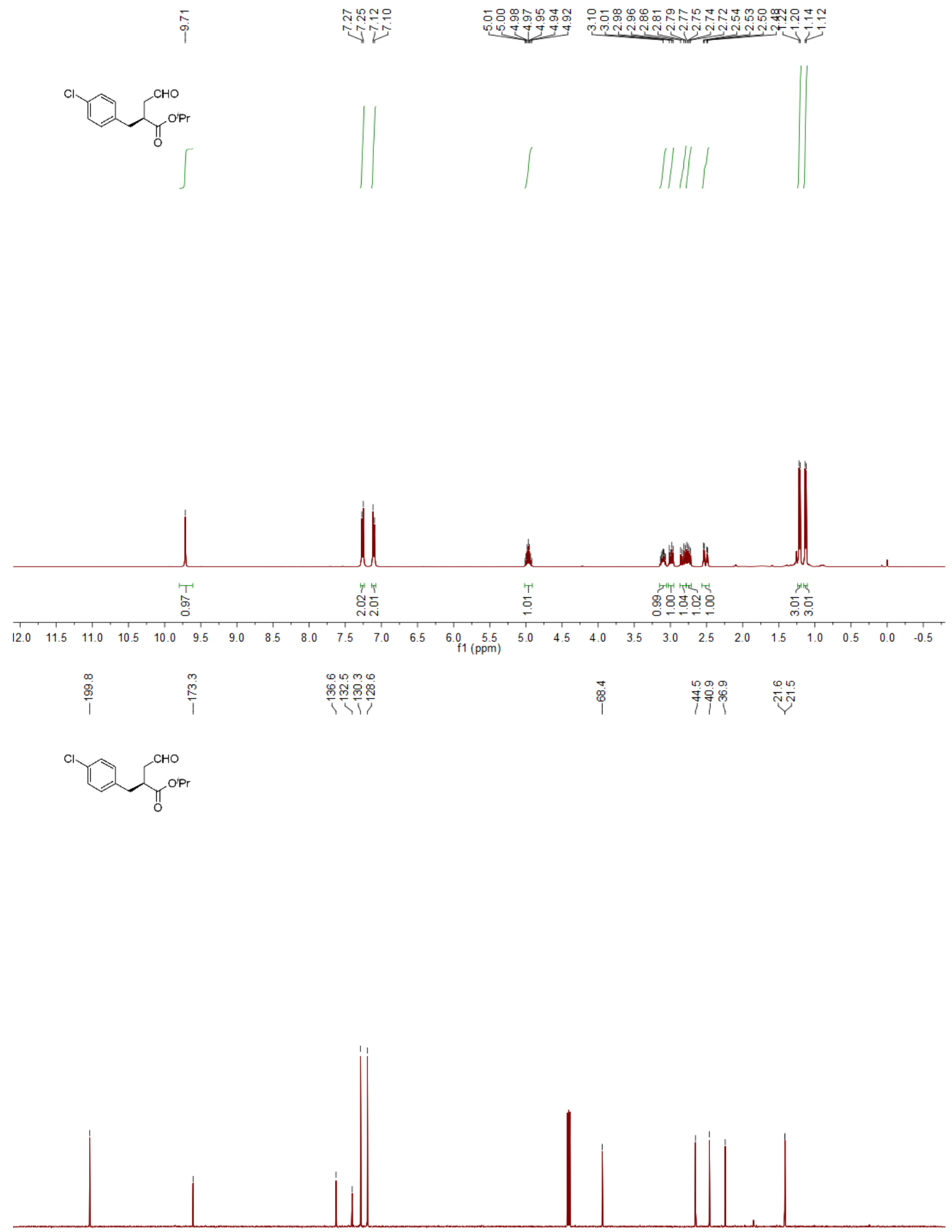

$\begin{array}{lllllllllllllllllllllllllllllll}210 & 200 & 190 & 180 & 170 & 160 & 150 & 140 & 130 & 120 & 110 & 100 & 90 & 80 & 70 & 60 & 50 & 40 & 30 & 20 & 10 & 0 & -10\end{array}$ 
OII

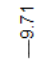

$\overbrace{\mathrm{O}}^{\mathrm{O} P \mathrm{Pr}}$

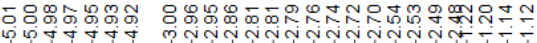

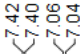

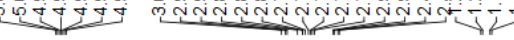

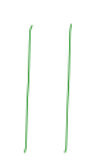

$\int / \|$

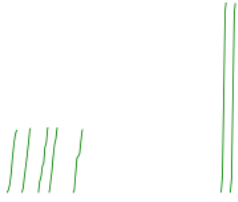

allitili

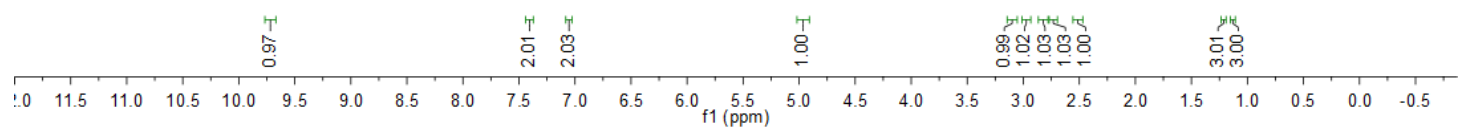

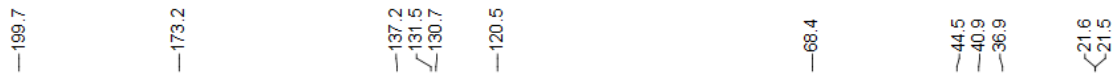

$\overbrace{0}^{\mathrm{CHO}}$

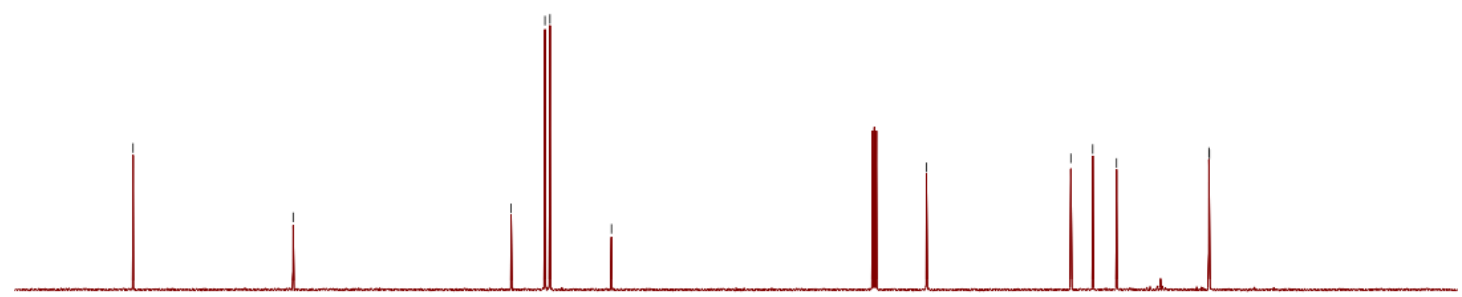

$\begin{array}{llllllllllllllllllllllll}210 & 200 & 190 & 180 & 170 & 160 & 150 & 140 & 130 & 120 & 110 & 100 & 90 & 80 & 70 & 60 & 50 & 40 & 30 & 20 & 10 & 0 & -10\end{array}$ 
<smiles>CCCOC(=O)C(CC=O)Cc1ccccc1OC</smiles>

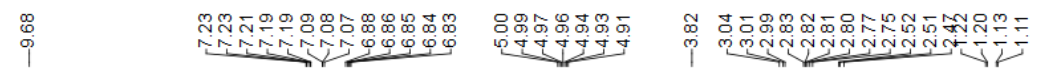<smiles>CC(=O)C(CO)Cc1ccccc1O</smiles><smiles>CC#CC(C)CC</smiles>
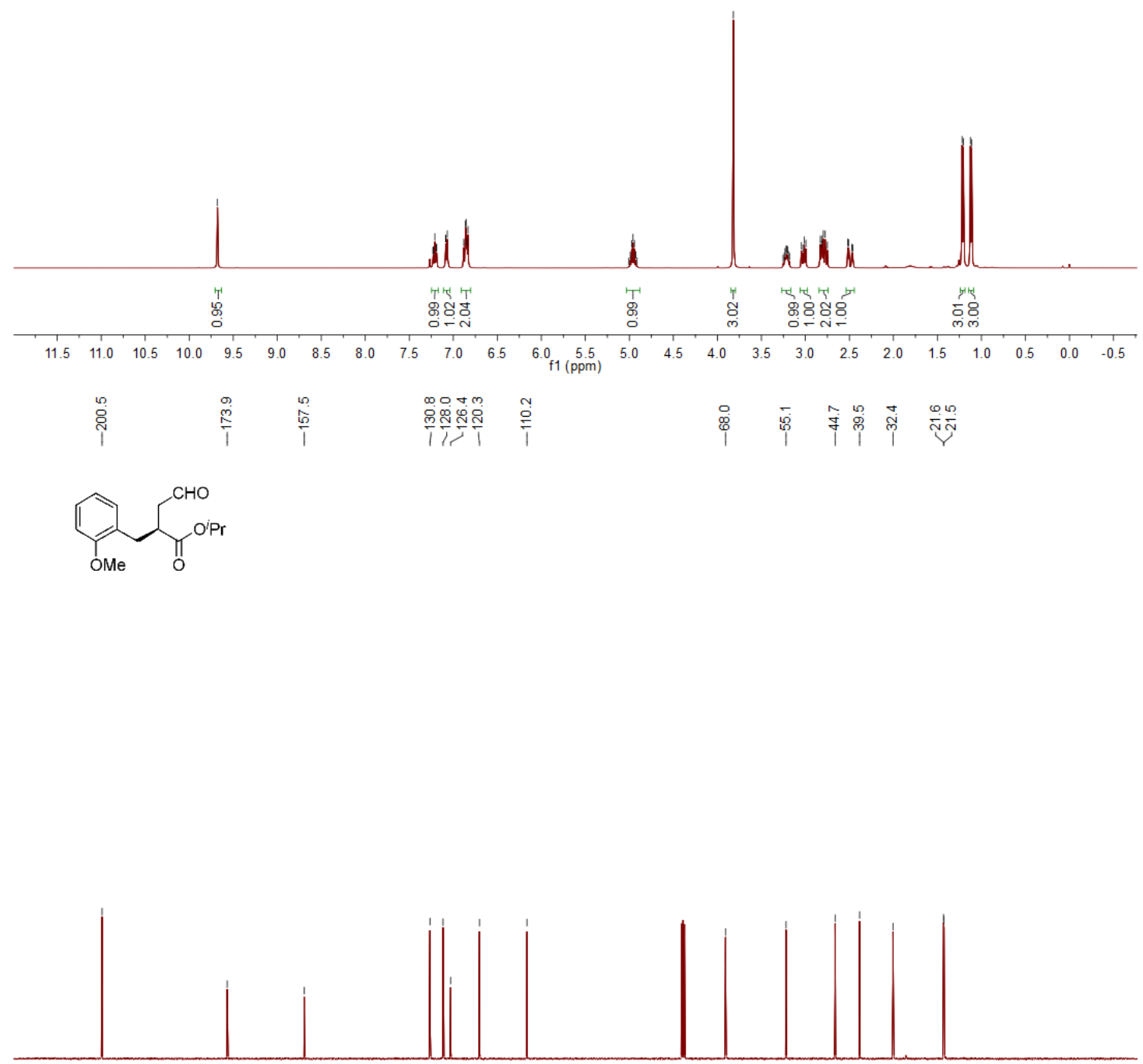

$\begin{array}{lllllllllllllllllllllll}210 & 200 & 190 & 180 & 170 & 160 & 150 & 140 & 130 & 120 & 110 & 100 & 90 & 80 & 70 & 60 & 50 & 40 & 30 & 20 & 10 & 0 & -10\end{array}$ 
<smiles>CCCCOC(=O)C(CC=O)Cc1cccc(OC)c1</smiles>

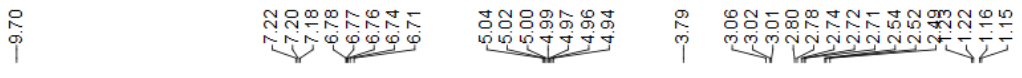<smiles>CCCC(=O)OC(CO)Cc1cccc(OC)c1</smiles>
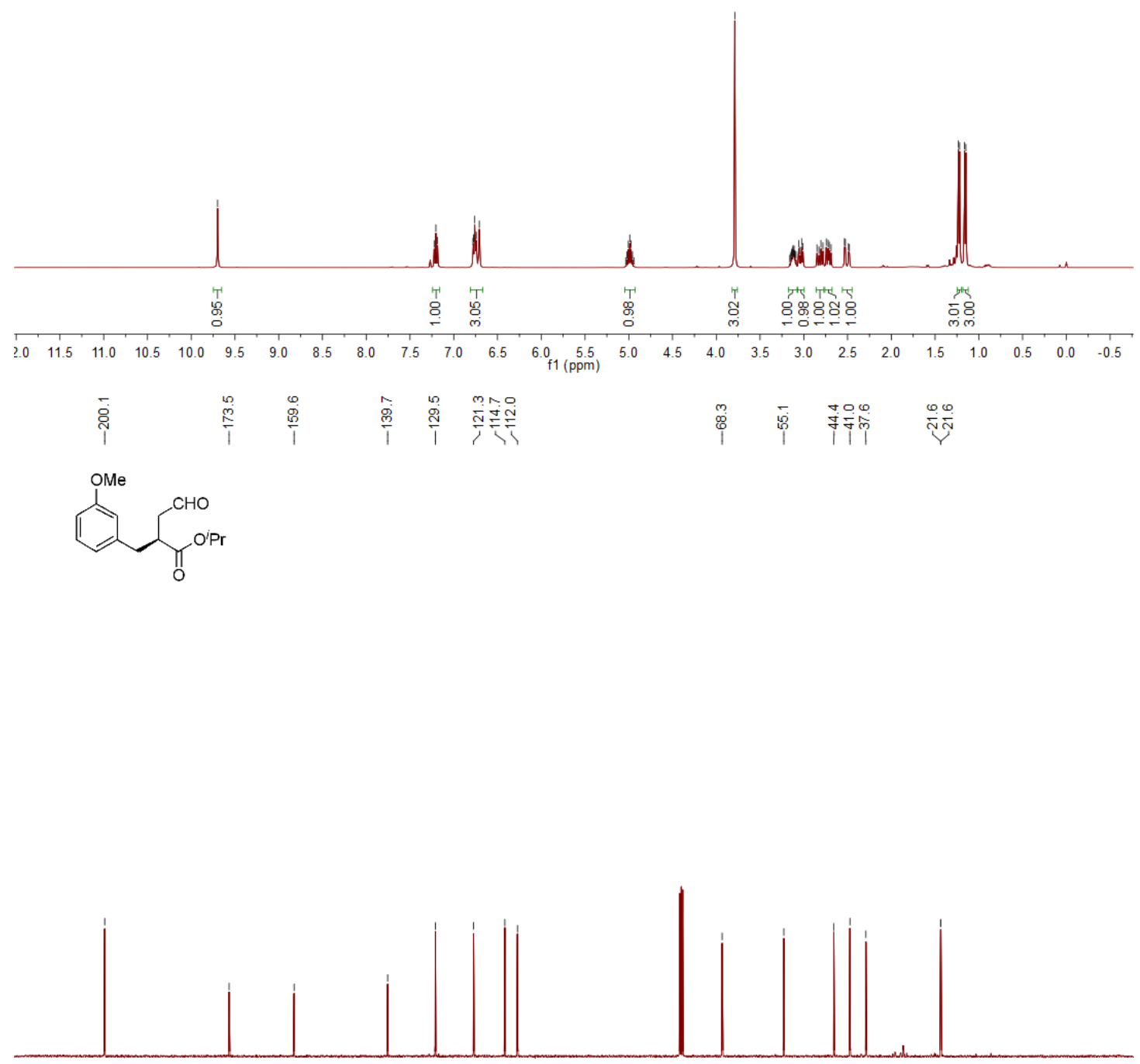

$\begin{array}{lllllllllllllllllllllllll}210 & 200 & 190 & 180 & 170 & 160 & 150 & 140 & 130 & 120 & 110 & 100 & 90 & 80 & 70 & 60 & 50 & 40 & 30 & 20 & 10 & 0 & -10\end{array}$ 

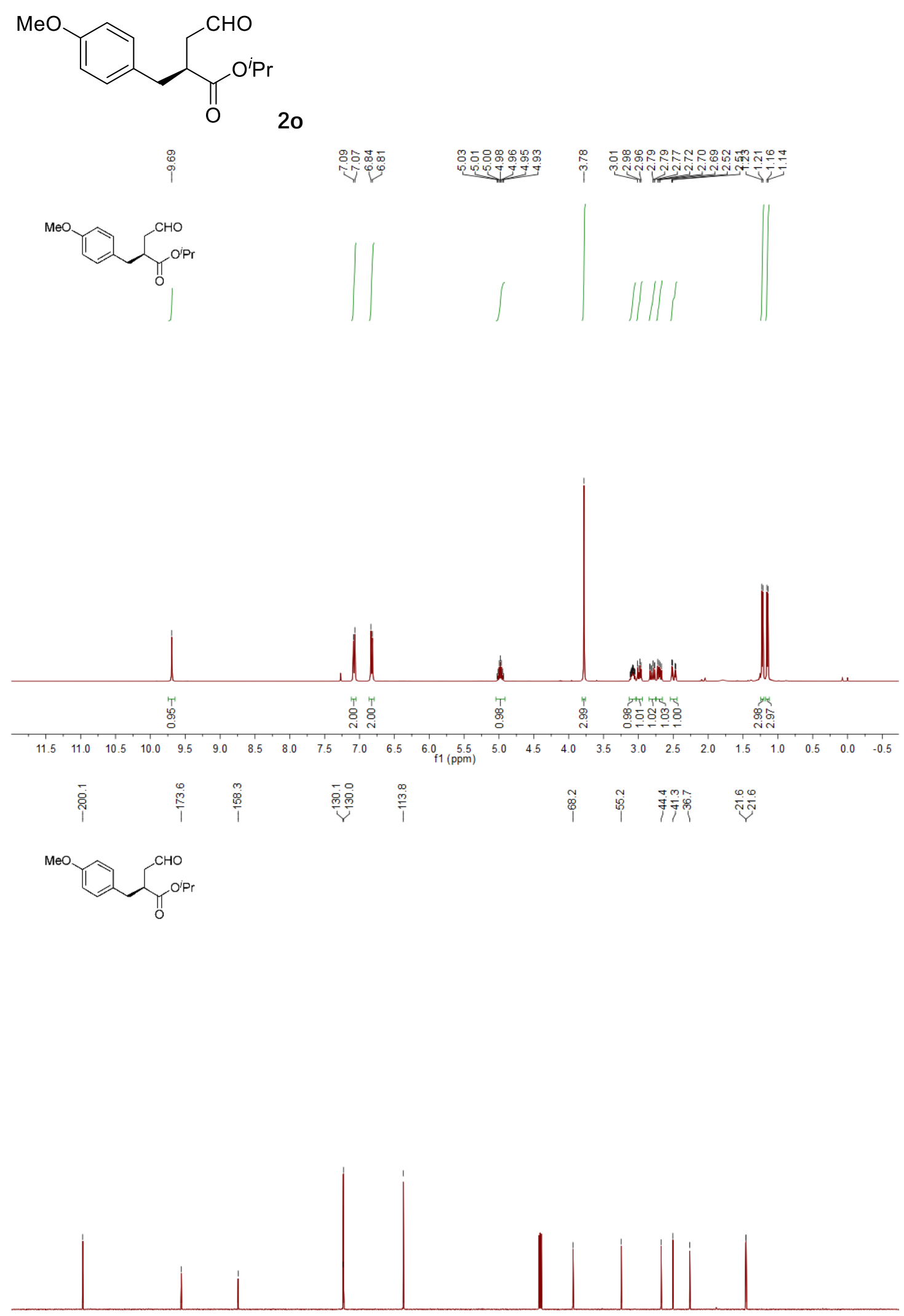

$\begin{array}{llllllllllllllllllllllllllllll}210 & 200 & 190 & 180 & 170 & 160 & 150 & 140 & 130 & 120 & 110 & 100 & 90 & 80 & 70 & 60 & 50 & 40 & 30 & 20 & 10 & 0 & -10\end{array}$ 
$\prod_{\mathrm{O}}^{\mathrm{O} O \mathrm{OEt}}$

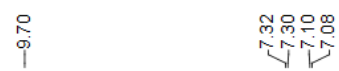

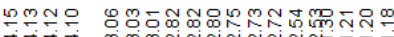

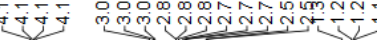

'Bu

.
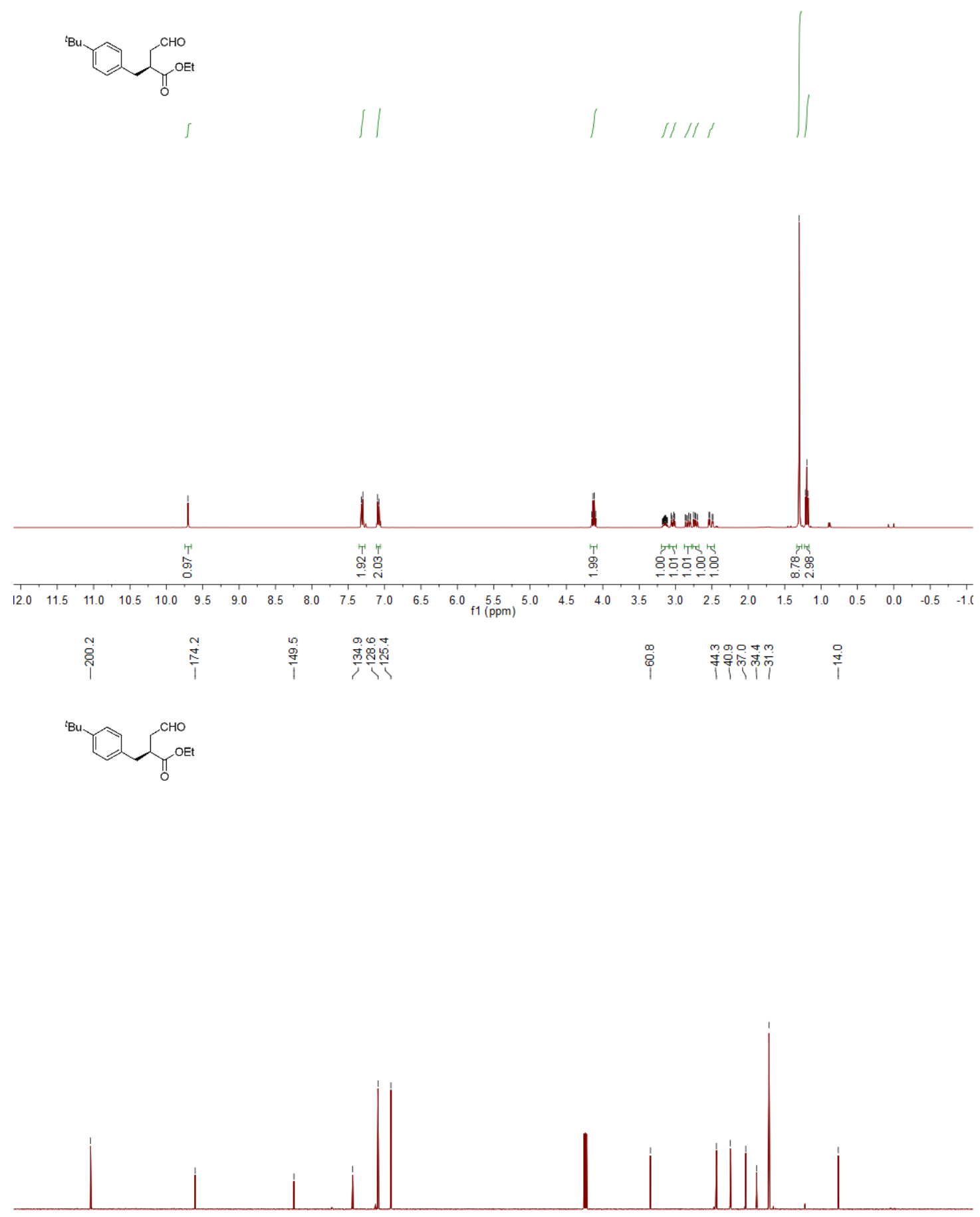

$\begin{array}{llllllllllllllllllllllllll}1 & 10 & 210 & 190 & 180 & 170 & 160 & 150 & 140 & 130 & 120 & 110 & 100 & 10 & 10 & 10 & 60 & 50 & 40 & 30 & 20 & 10 & 0 & -10\end{array}$ 

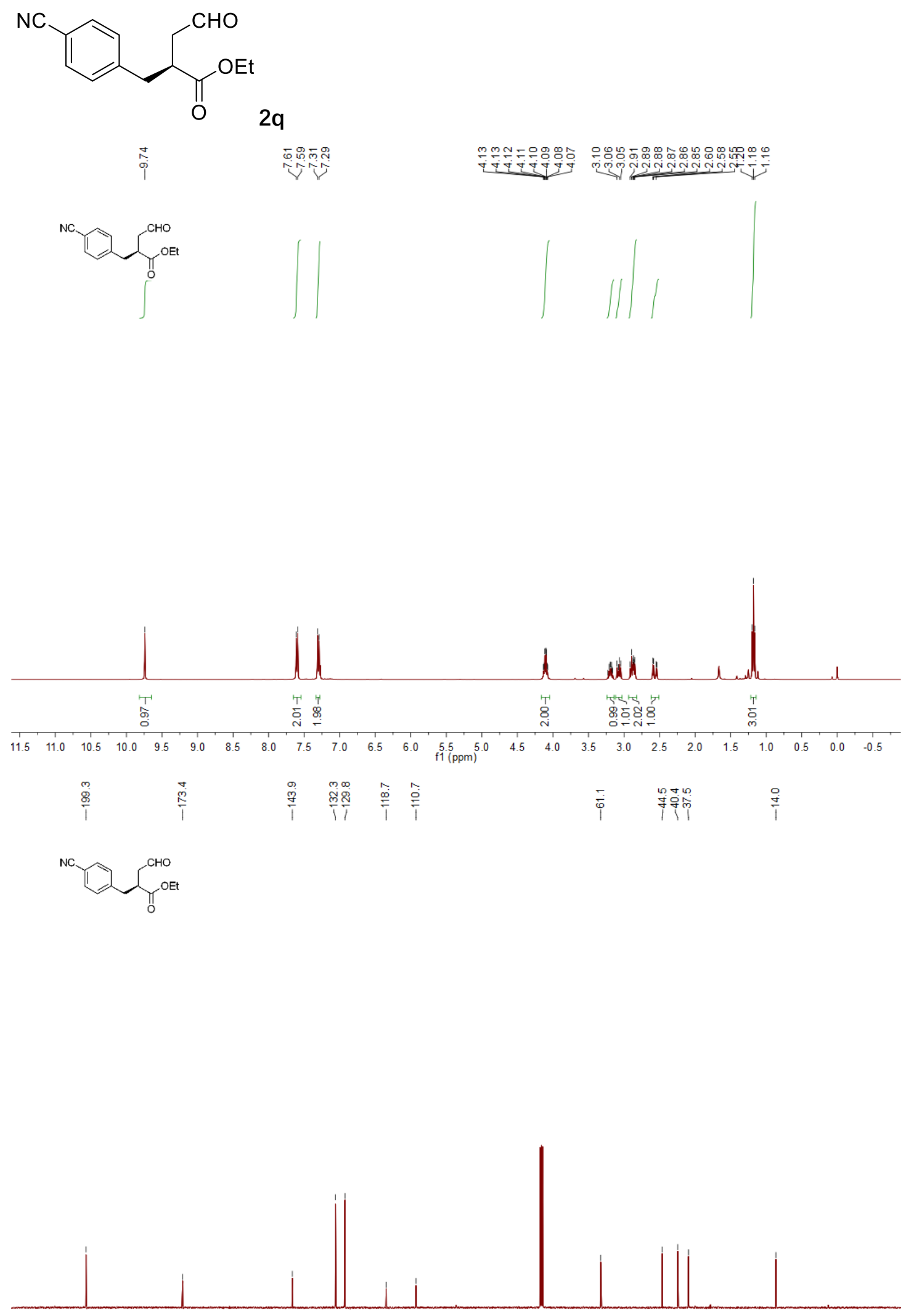

$\begin{array}{llllllllllllllllllllllllll}210 & 200 & 190 & 180 & 170 & 160 & 150 & 140 & 130 & 120 & 110 & 100 & 10 & 80 & 70 & 60 & 50 & 40 & 30 & 20 & 10 & 0 & -10\end{array}$ 


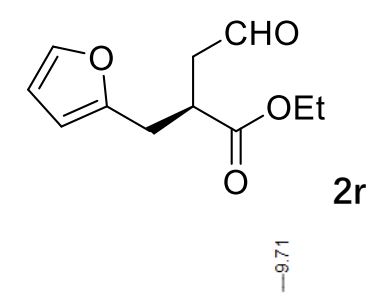

$\overbrace{0}^{\text {CHO }}$

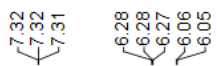

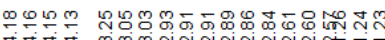

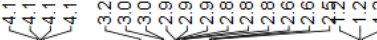

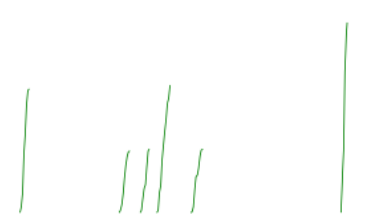

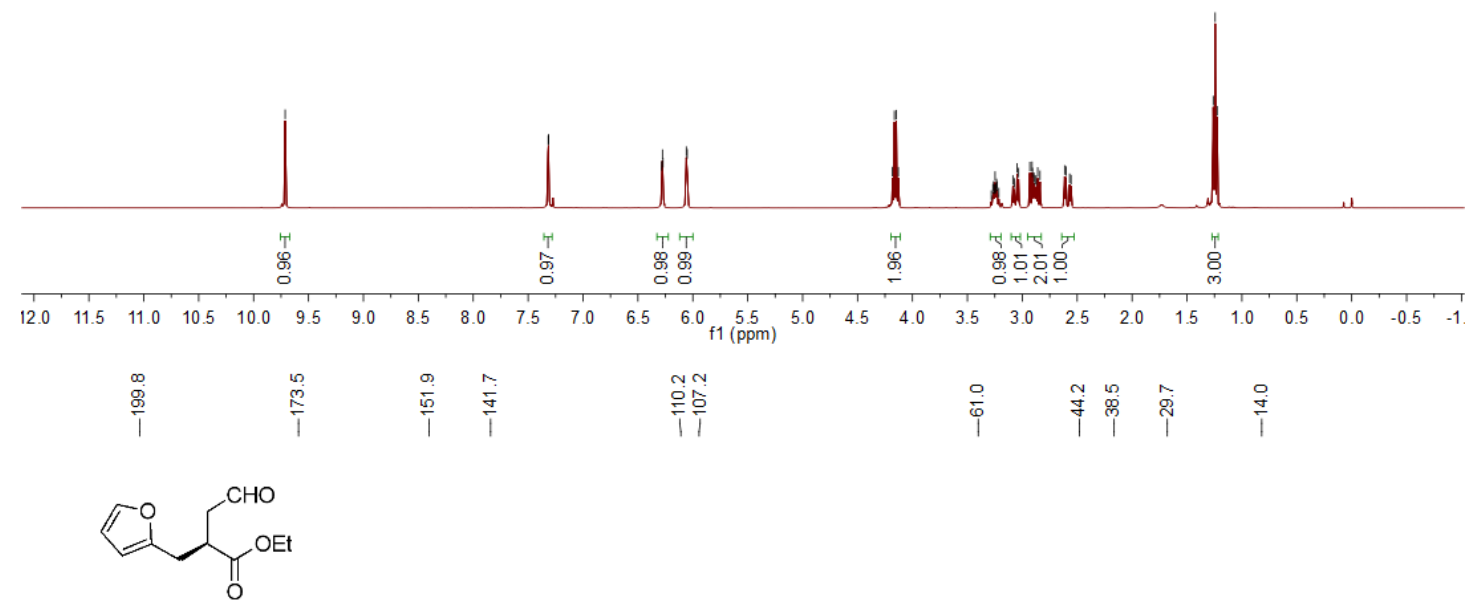

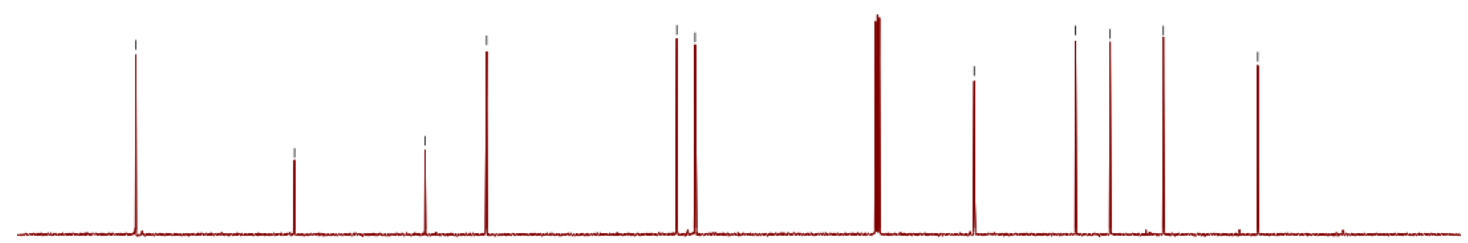

$\begin{array}{lllllllllllllllllllllllll}210 & 200 & 190 & 180 & 170 & 160 & 150 & 140 & 130 & 120 & 110 & 100 & 90 & 80 & 70 & 60 & 50 & 40 & 30 & 20 & 10 & 0 & -10\end{array}$ 
$\prod_{\mathrm{O}}^{\mathrm{CHO}}$

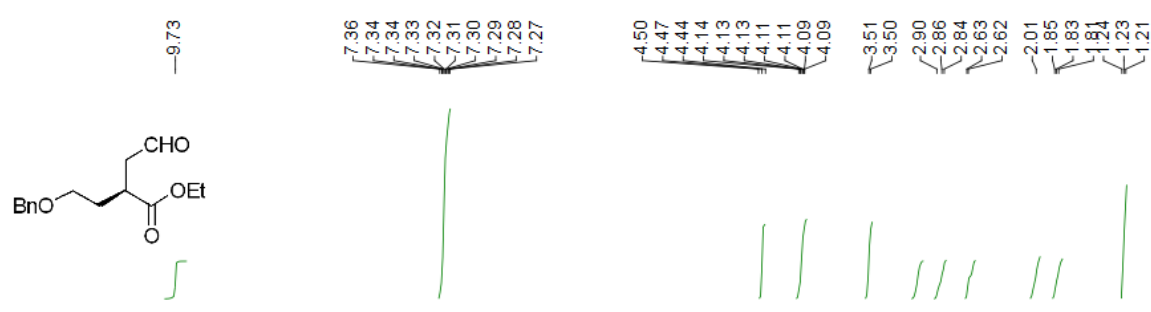

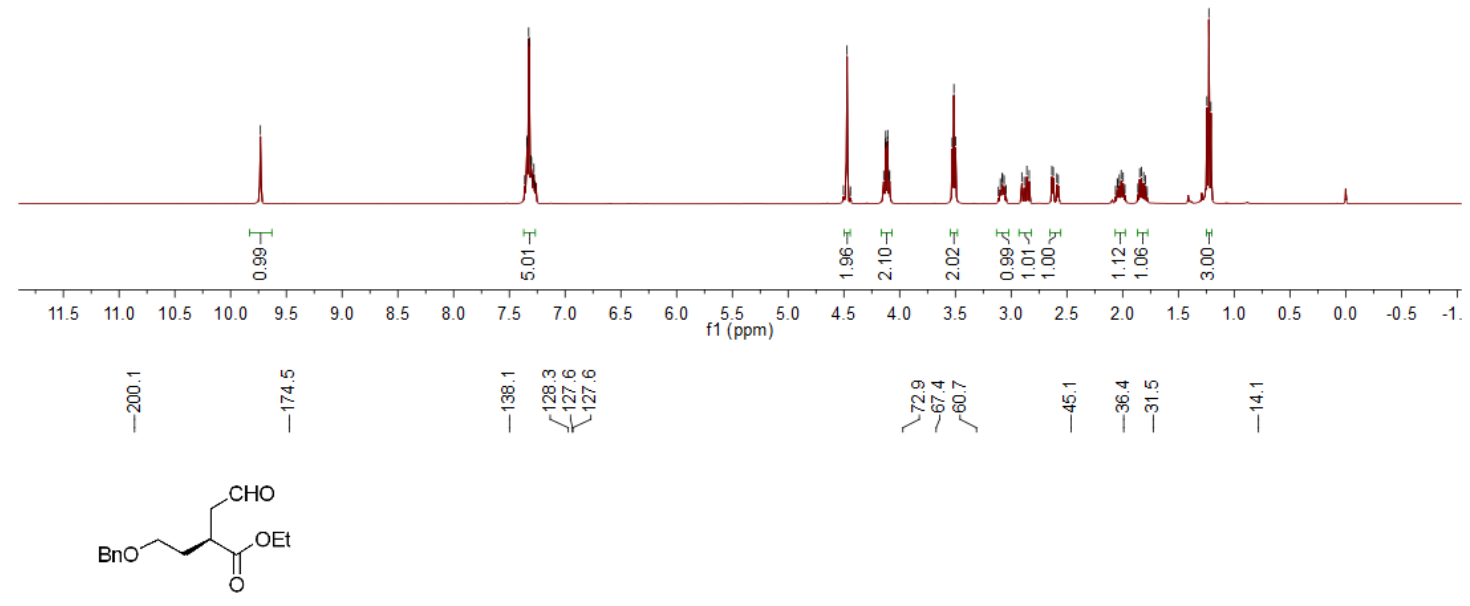

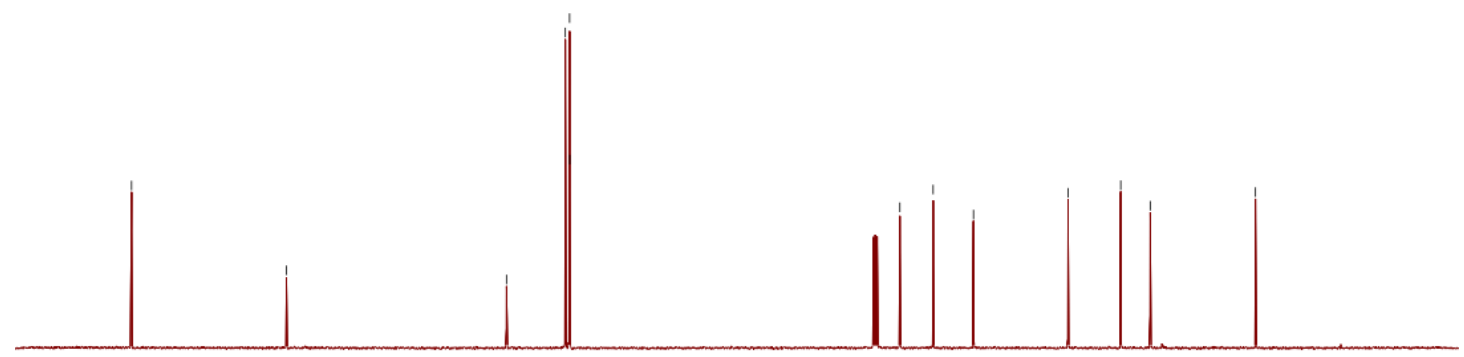

$\begin{array}{lllllllllllllllllllllllllll}210 & 200 & 190 & 180 & 170 & 160 & 150 & 140 & 130 & 120 & 110 & 100 & 90 & 80 & 70 & 60 & 50 & 40 & 30 & 20 & 10 & 0 & -10\end{array}$ 


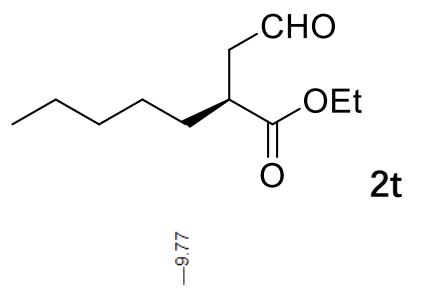

$\stackrel{\circ}{0}=\frac{0}{-10}$

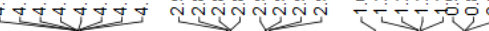
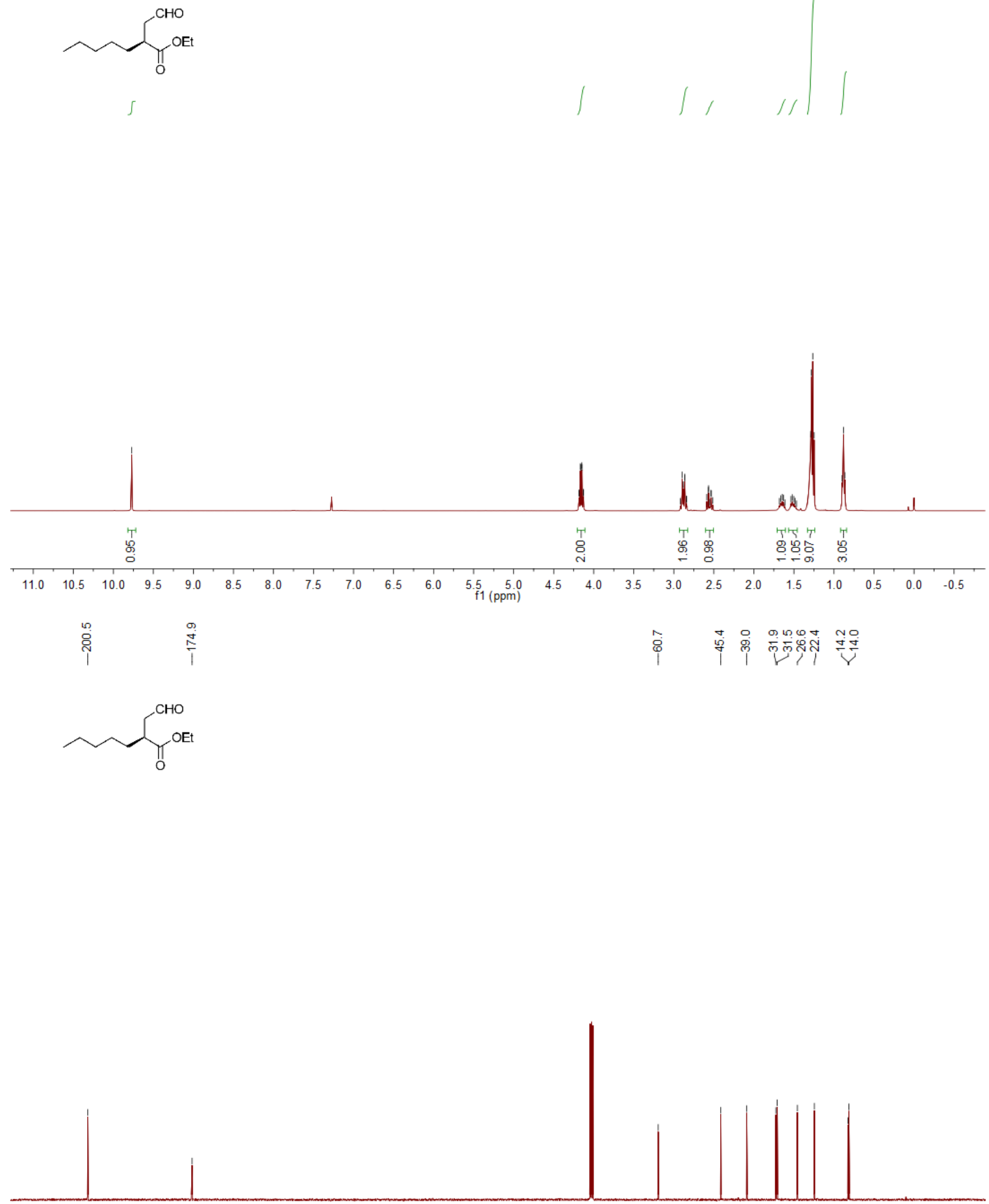

$\begin{array}{llllllllllllllllllllllllll}210 & 200 & 190 & 180 & 170 & 160 & 150 & 140 & 130 & 120 & 110 & 100 & 90 & 80 & 70 & 60 & 50 & 40 & 30 & 20 & 10 & 0 & -10\end{array}$ 


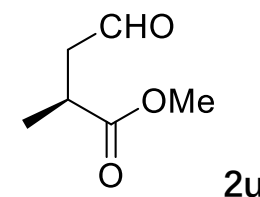

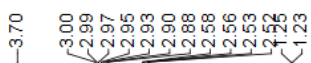
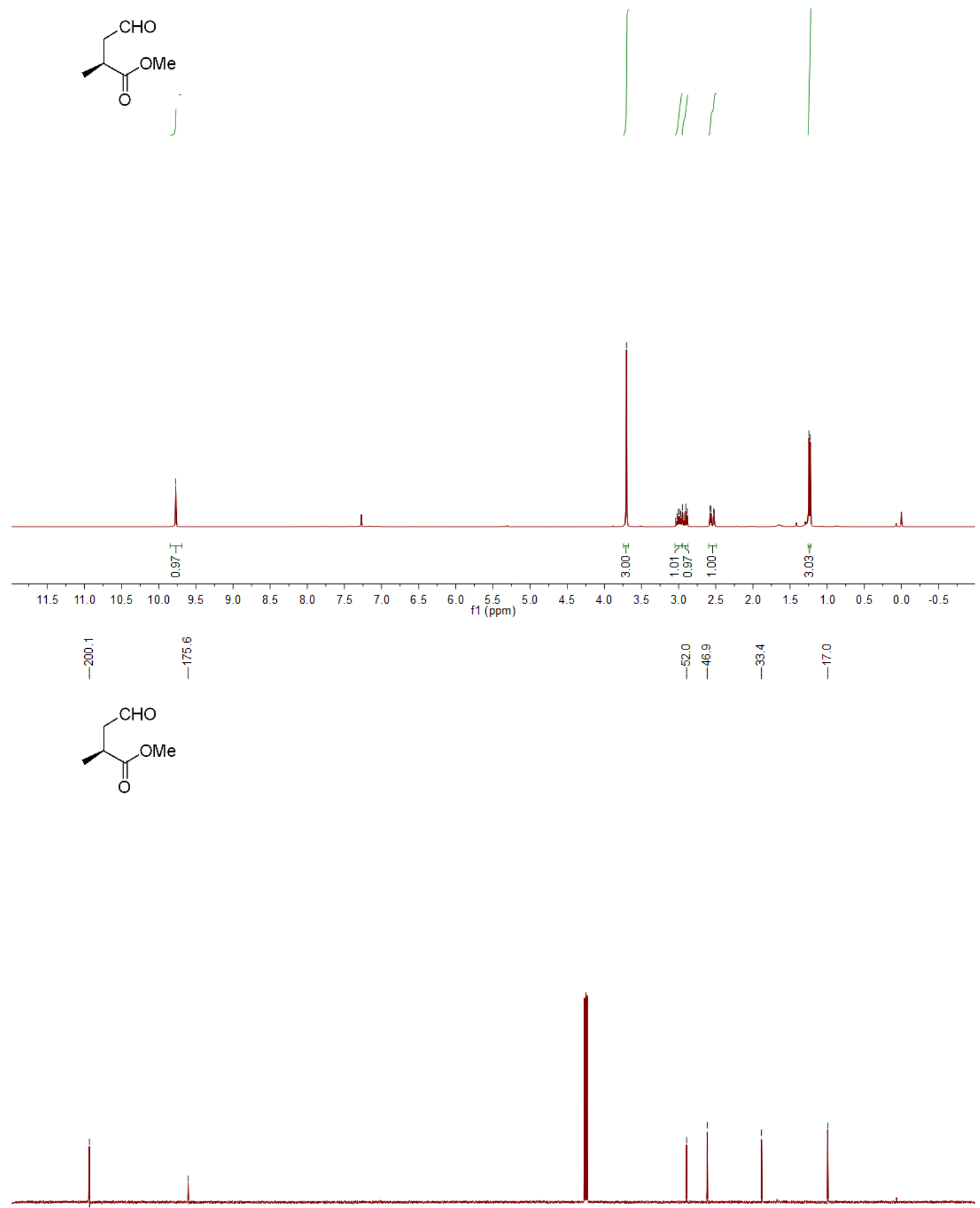

\begin{tabular}{lllllllllllllllllllllllll}
\hline 210 & 200 & 190 & 180 & 170 & 160 & 150 & 140 & 130 & 120 & 110 & 100 & 10 & 10 & 10 & 60 & 50 & 40 & 30 & 20 & 10 & 0 & -10
\end{tabular} 
<smiles>COC(=O)C[C@H](CC=O)C(=O)OC</smiles>

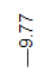

Meo $\overbrace{0}^{\mathrm{N}} \mathrm{C}_{0}^{\mathrm{OM}}$
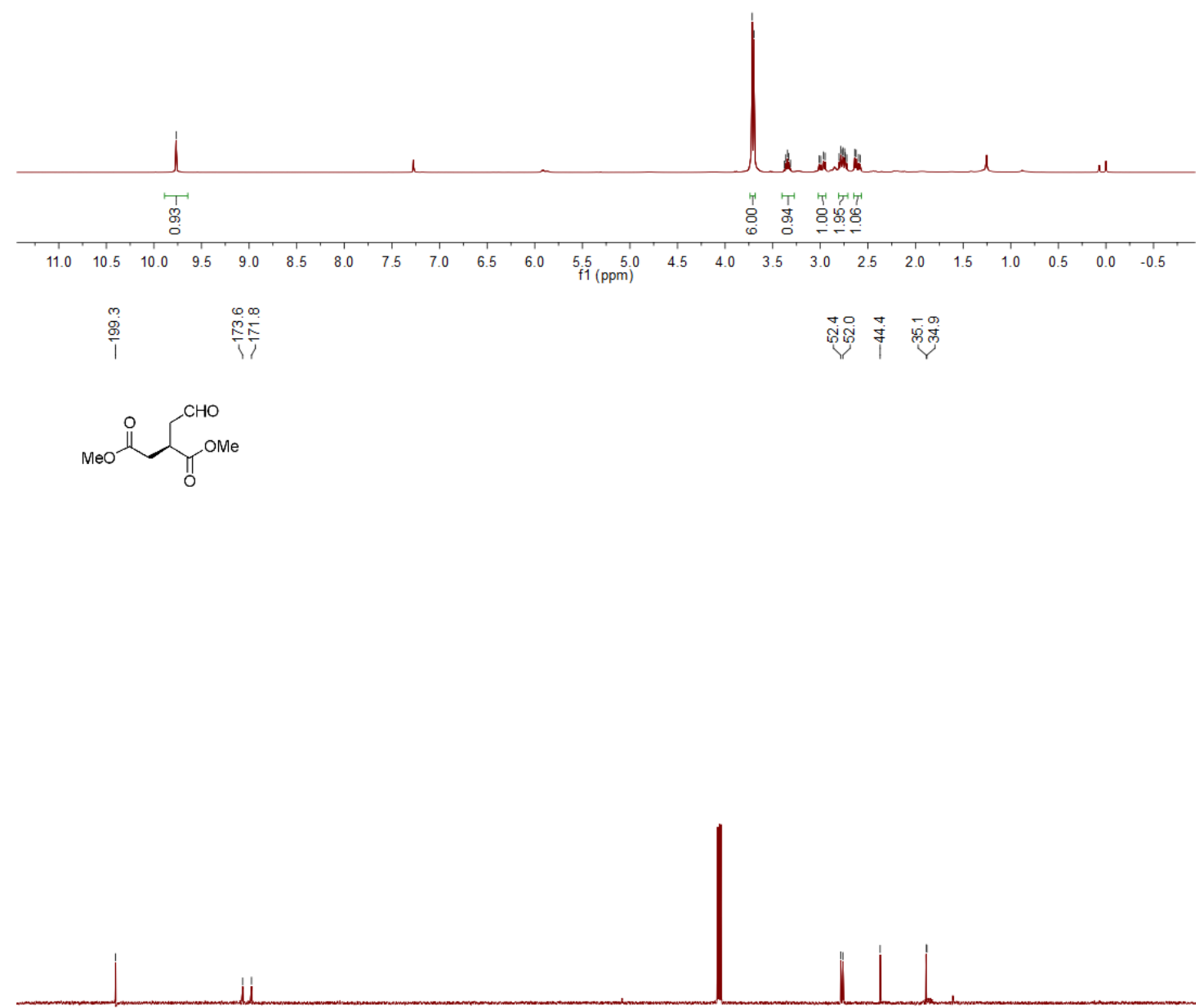

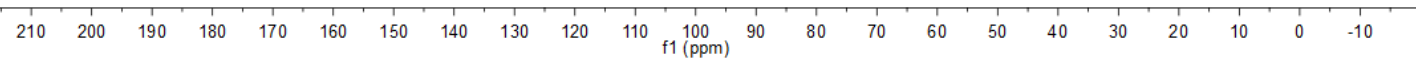




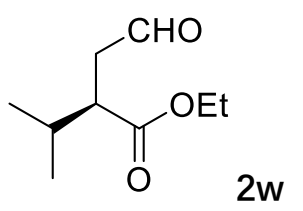

$\stackrel{\substack{\infty \\ \infty}}{\substack{1 \\ i}}$

ำ

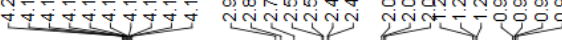<smiles>CCOC(=O)C(CC=O)C(C)C</smiles>
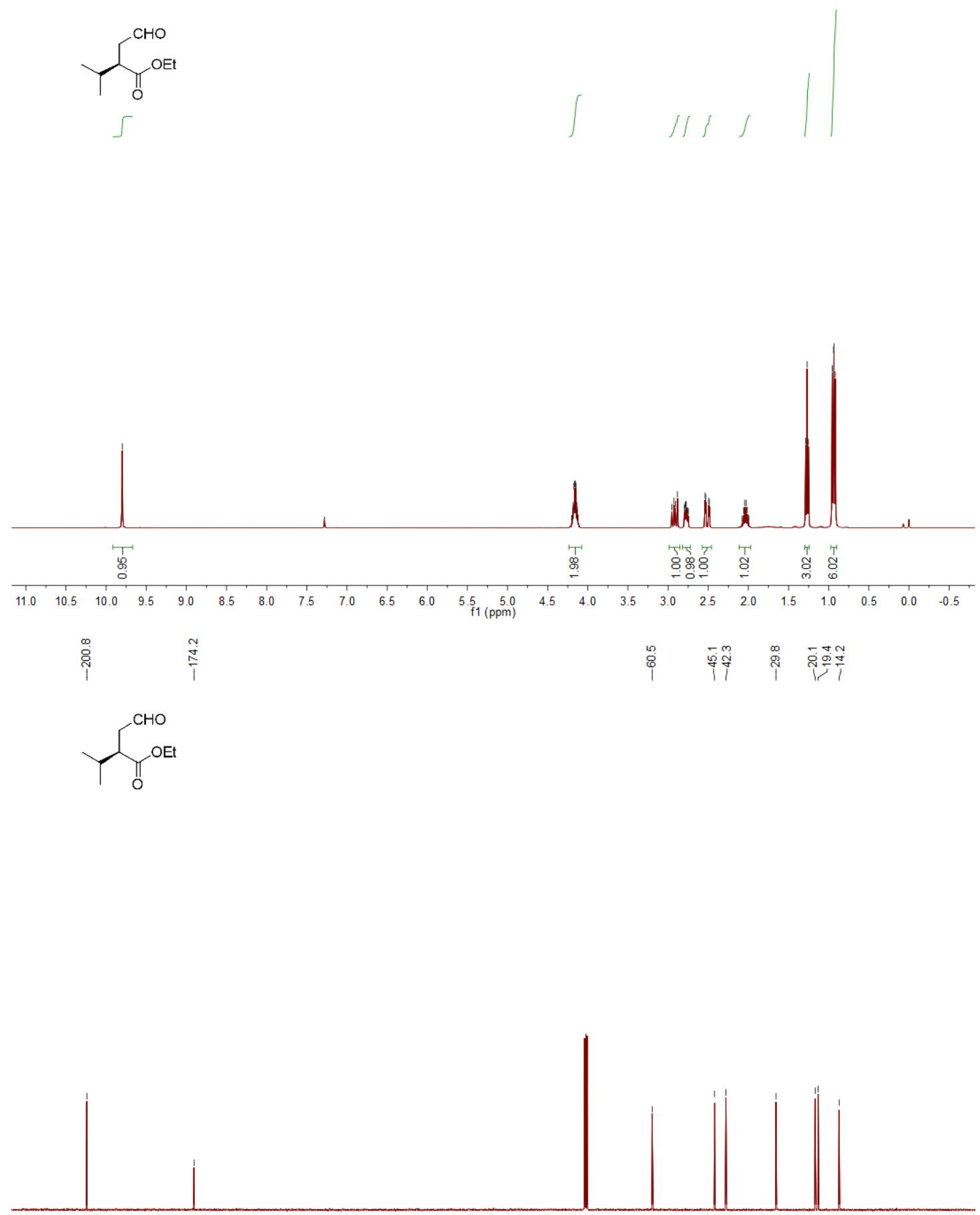

$\begin{array}{lllllllllllllllllllllllll}210 & 200 & 190 & 180 & 170 & 160 & 150 & 140 & 130 & 120 & 110 & 100 & 90 & 80 & 70 & 60 & 50 & 40 & 30 & 20 & 10 & 0 & -10\end{array}$ 
$\overbrace{4 a}$

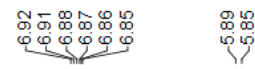

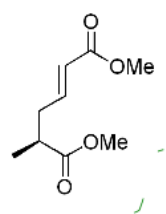

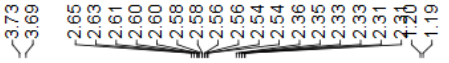

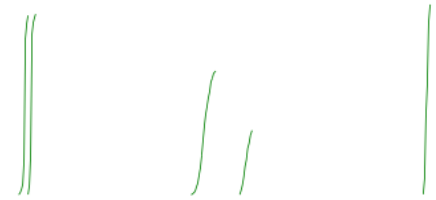

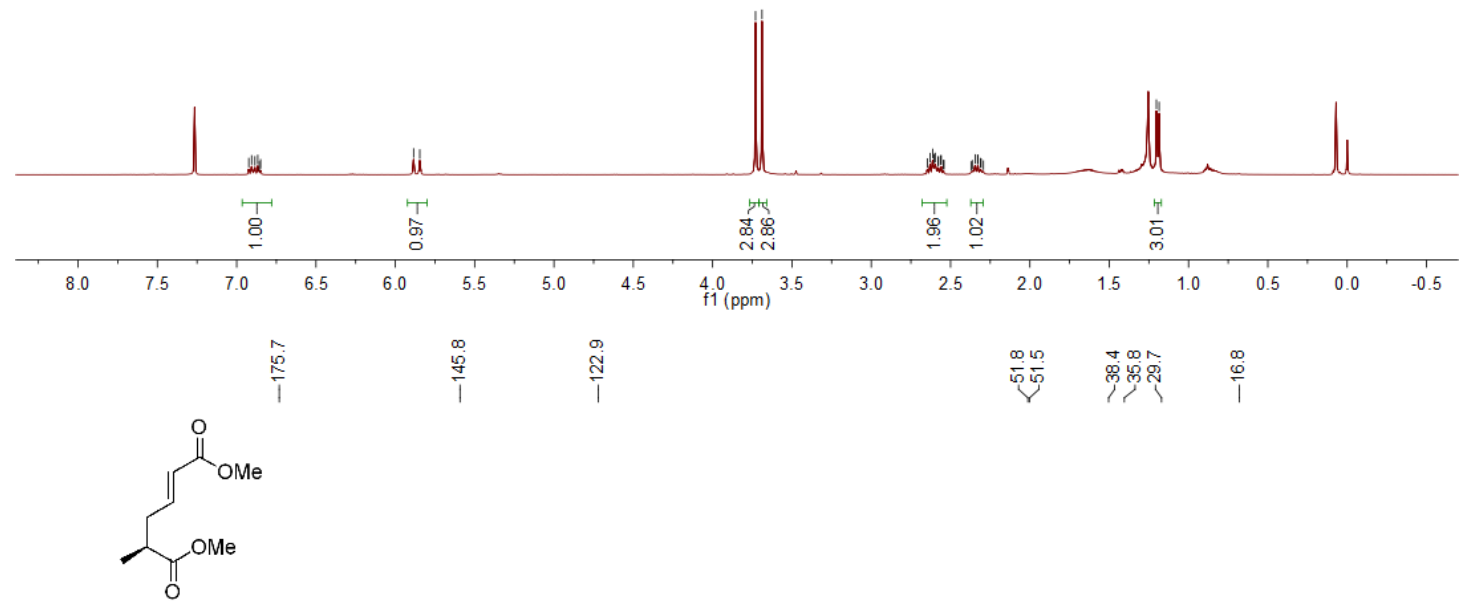

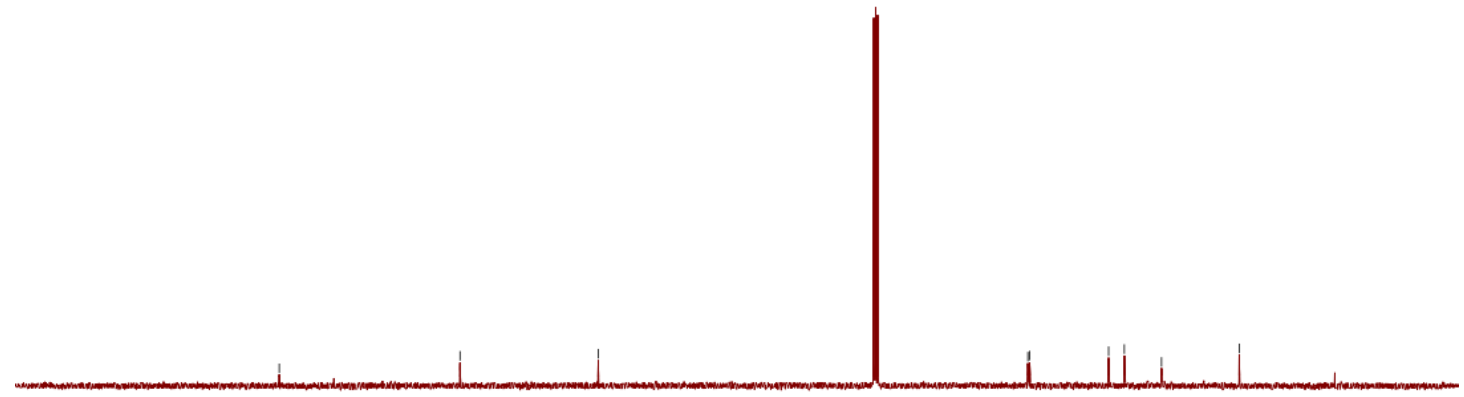

$\begin{array}{llllllllllllllllllllllllllll}210 & 200 & 190 & 180 & 170 & 160 & 150 & 140 & 130 & 120 & 110 & 100 & 90 & 80 & 70 & 60 & 50 & 40 & 30 & 20 & 10 & 0 & -10\end{array}$ 
$\overbrace{0}^{0}$

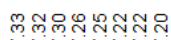

证证等<smiles>COC(=O)C(CCO)Cc1ccccc1</smiles>

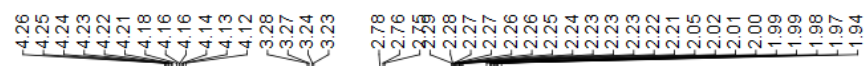
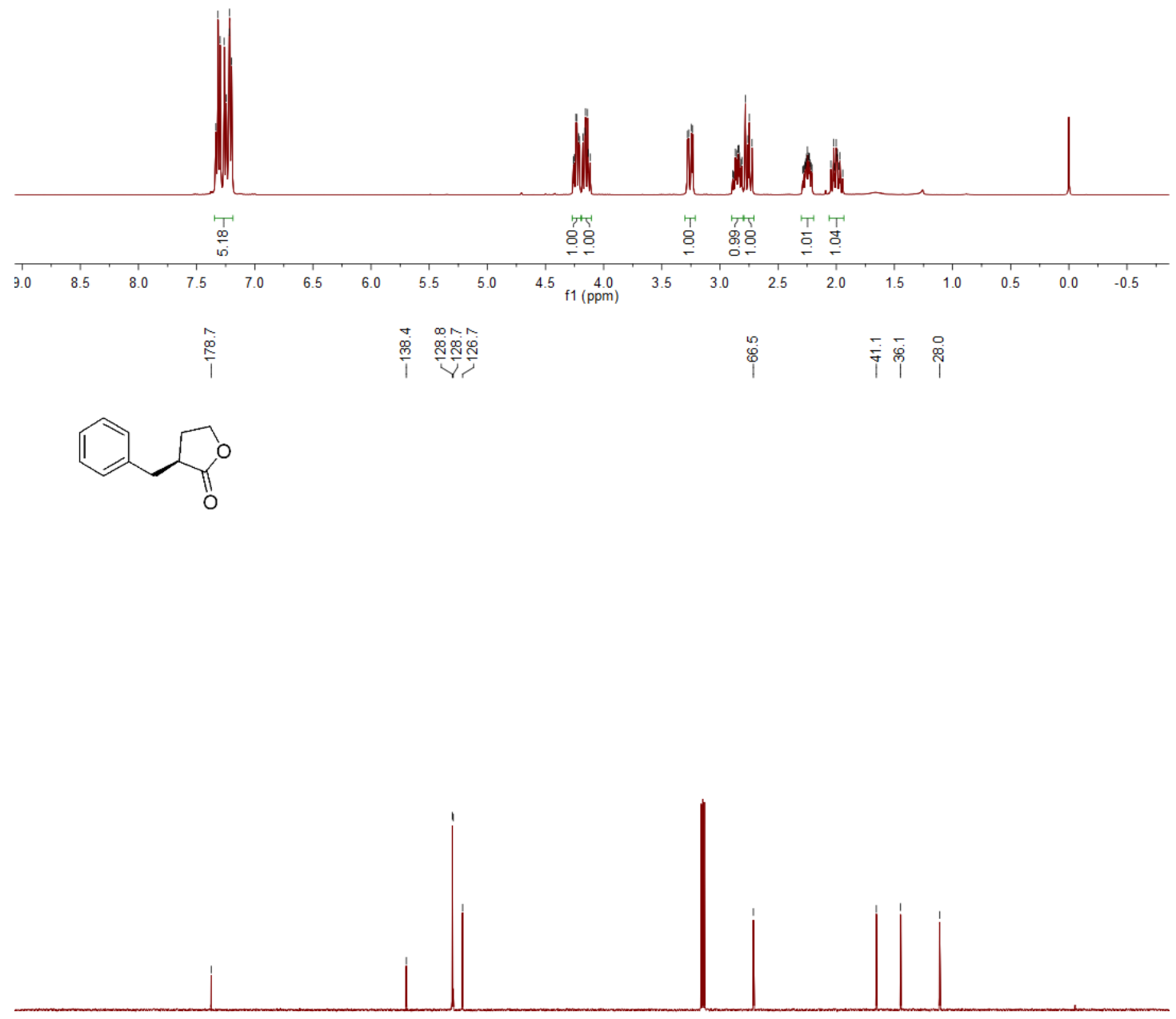

$\begin{array}{llllllllllllllllllllllllll}210 & 200 & 190 & 180 & 170 & 160 & 150 & 140 & 130 & 120 & 110 & 100 & 10 & 80 & 70 & 60 & 50 & 40 & 30 & 20 & 10 & 0 & -10\end{array}$ 


\section{HPLC spectra}<smiles>[R]OC(=O)[C@H](CC=O)Cc1ccccc1</smiles>

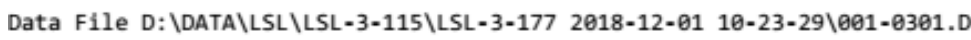
Sample Name: OET RAC
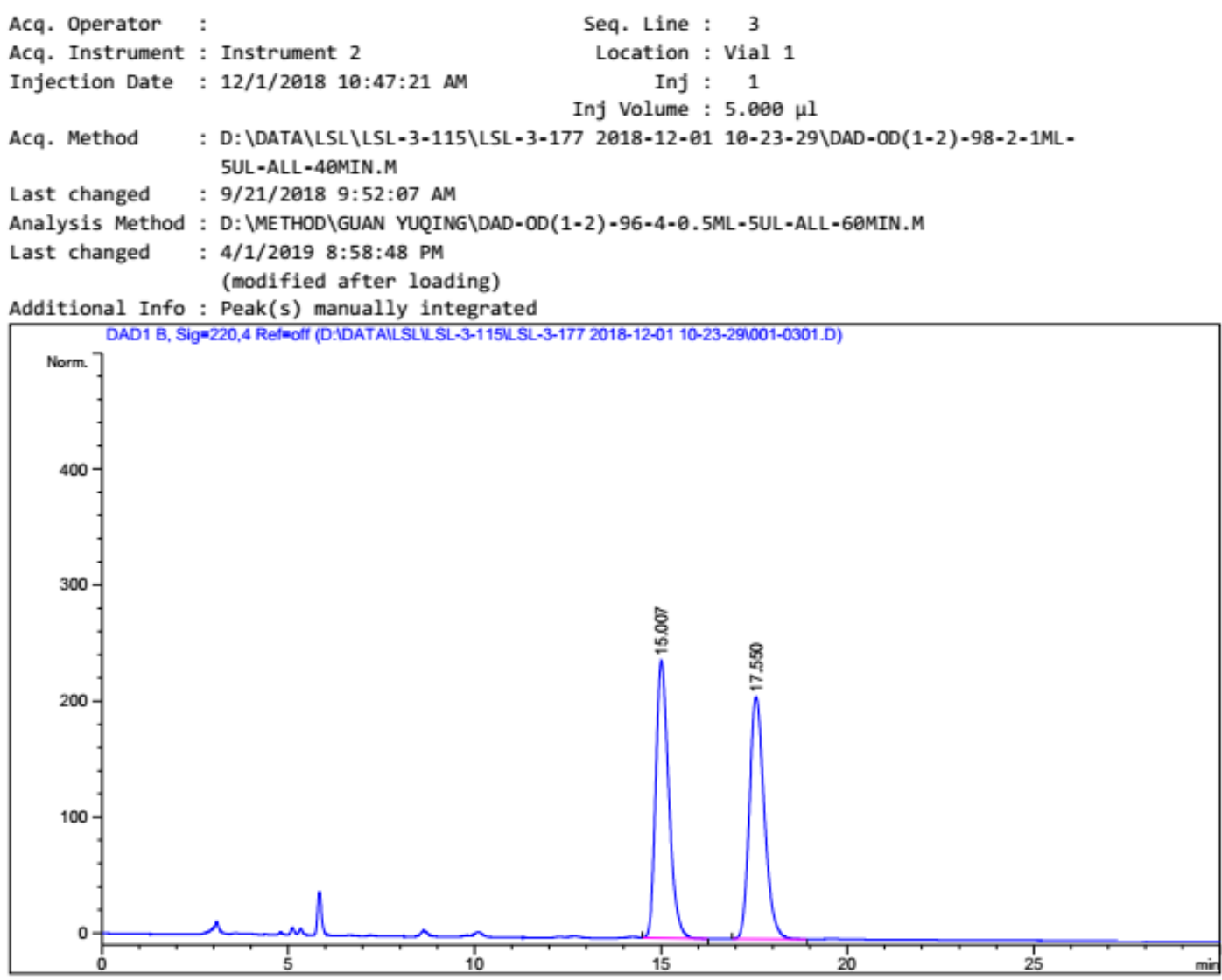

Area Percent Report

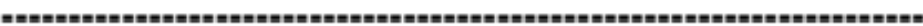

$\begin{array}{lll}\text { Sorted By } & : & \text { Signal } \\ \text { Multiplier } & : & 1.0000 \\ \text { Dilution } & : & 1.0000\end{array}$

Use Multiplier \& Dilution Factor with ISTDs

Signal 1: DAD1 B, Sig=220,4 Ref=off

\begin{tabular}{|c|c|c|c|c|c|c|}
\hline $\begin{array}{c}\text { Peak } \\
\#\end{array}$ & $\begin{array}{c}\text { RetTime } \\
\text { [min] }\end{array}$ & Type & $\begin{array}{l}\text { Width } \\
\text { [min] }\end{array}$ & $\begin{array}{c}\text { Area } \\
{\left[\mathrm{mAU}^{*} \mathrm{~s}\right]}\end{array}$ & $\begin{array}{l}\text { Height } \\
\text { [mAU] }\end{array}$ & $\begin{array}{c}\text { Area } \\
\%\end{array}$ \\
\hline$\cdots$ & ne. & 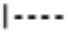 & . & 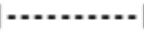 & and & (2).e. \\
\hline 1 & 15.007 & BB & 0.3727 & 5802.45654 & 239.45332 & 49.9077 \\
\hline 2 & 17.550 & BB & 0.4315 & 5823.92432 & 208.76959 & 50.0923 \\
\hline Tota & : & & & $1.16264 \mathrm{e} 4$ & 448.22292 & \\
\hline
\end{tabular}


Data File D: \DATA \LSL\LSL-3-115\LSL-3-177 2018-12-01 10-23-29\002-0401.D Sample Name: LSL-3-177-1

\begin{tabular}{|c|c|c|}
\hline Acq. Operator & : & Seq. Line : 4 \\
\hline Acq. Instrument & : Instrument 2 & Location : Vial 2 \\
\hline Injection Date & : 12/1/2018 11:28:20 AM & $\begin{array}{rlc}\text { Inj } & : & 1 \\
\text { Inj Volume } & : & 5.000\end{array}$ \\
\hline
\end{tabular}

Acq. Method : D: \DATA \LSL\LSL-3-115\LSL-3-177 2018-12-01 10-23-29 \DAD-OD(1-2)-98-2-1ML-

$\begin{aligned} & 5 U L-A L L-40 M I N . M \\ & \text { Last changed } \quad: \quad 9 / 21 / 2018 \text { 9:52:07 AM }\end{aligned}$

Analysis Method : D: \METHOD \GUAN YUQING

Last changed : 4/1/2019 9:02:28 PM

(modified after loading)

Additional Info : Peak(s) manually integrated

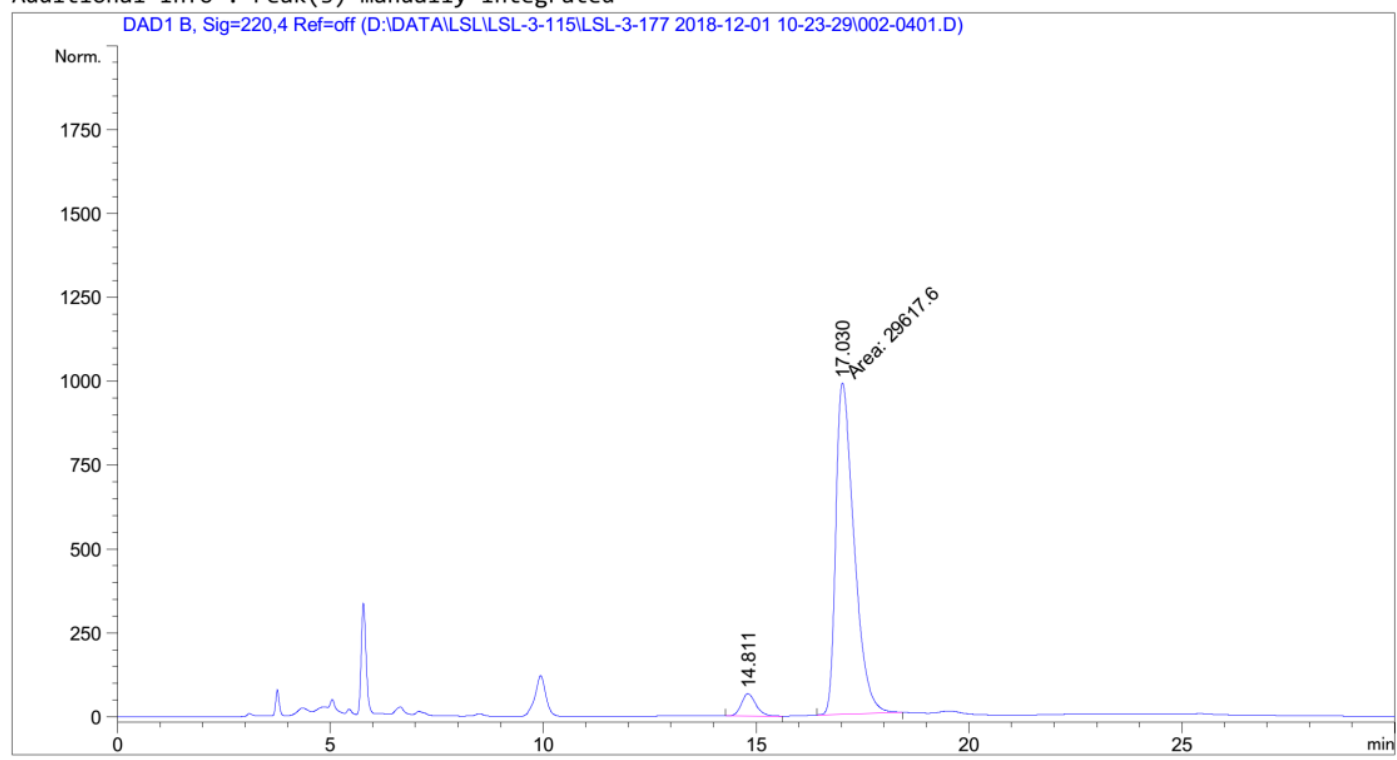

Area Percent Report

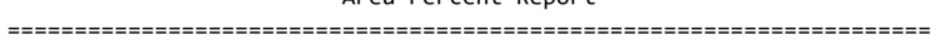

$\begin{array}{lll}\text { Sorted By } & : & \text { Signal } \\ \text { Multiplier } & : & 1.0000 \\ \text { Dilution } & : & 1.0000\end{array}$

Use Multiplier \& Dilution Factor with ISTDs

Signal 1: DAD1 B, Sig=220,4 Ref=off

\begin{tabular}{|c|c|c|c|c|c|}
\hline $\begin{array}{c}\text { Peak } \\
\text { \# }\end{array}$ & $\begin{array}{l}\text { RetTime Type } \\
\text { [min] }\end{array}$ & $\begin{array}{l}\text { Width } \\
\text { [min] }\end{array}$ & $\begin{array}{c}\text { Area } \\
{[\mathrm{mAU} * \mathrm{~s}]}\end{array}$ & $\begin{array}{l}\text { Height } \\
\text { [mAU] }\end{array}$ & $\begin{array}{c}\text { Area } \\
\%\end{array}$ \\
\hline---1 & $|-\ldots-n|--$ & $\ldots$ & | - . & - & $-\ldots$ \\
\hline 1 & 14.811 ВВ & 0.3597 & 1558.74170 & 65.96465 & 4.9998 \\
\hline 2 & $17.030 \mathrm{MM}$ & 0.4993 & $2.96176 \mathrm{e} 4$ & 988.58087 & 95.0002 \\
\hline Total & : & & $3.11764 \mathrm{e} 4$ & 1054.54552 & \\
\hline
\end{tabular}


<smiles>COC(=O)C(CC=O)Cc1ccccc1</smiles>

Data File D: \DATA \XZC\XZC-DATA-5\XZC-20190403-1 2019-04-03 14-46-28\031-1201.D Sample Name: LSL-ME RAC

\begin{tabular}{|c|c|c|}
\hline Acq. Operator & : & Seq. Line : 12 \\
\hline Acq. Instrument & : Instrument 2 & Location : Vial 31 \\
\hline Injection Date & : 4/4/2019 12:49:02 AM & $\begin{array}{rcc}\text { Inj } & : & 1 \\
\text { Inj Volume } & : & 5.000 \mu\end{array}$ \\
\hline
\end{tabular}

Acq. Method : D: \DATA \XZC \XZC-DATA-5 \XZC-20190403-1 2019-04-03 14-46-28\DAD-OD(1-2)-98-21ML-5UL-ALL-40MIN.M

Last changed : 9/21/2018 9:52:07 AM

Analysis Method: D: \METHOD\GUAN YUQING \DAD-OD(1-2)-96-4-0.5ML-5UL-ALL-120MIN.M

Last changed : 4/4/2019 8:55:29 AM

(modified after loading)

Additional Info : Peak(s) manually integrated

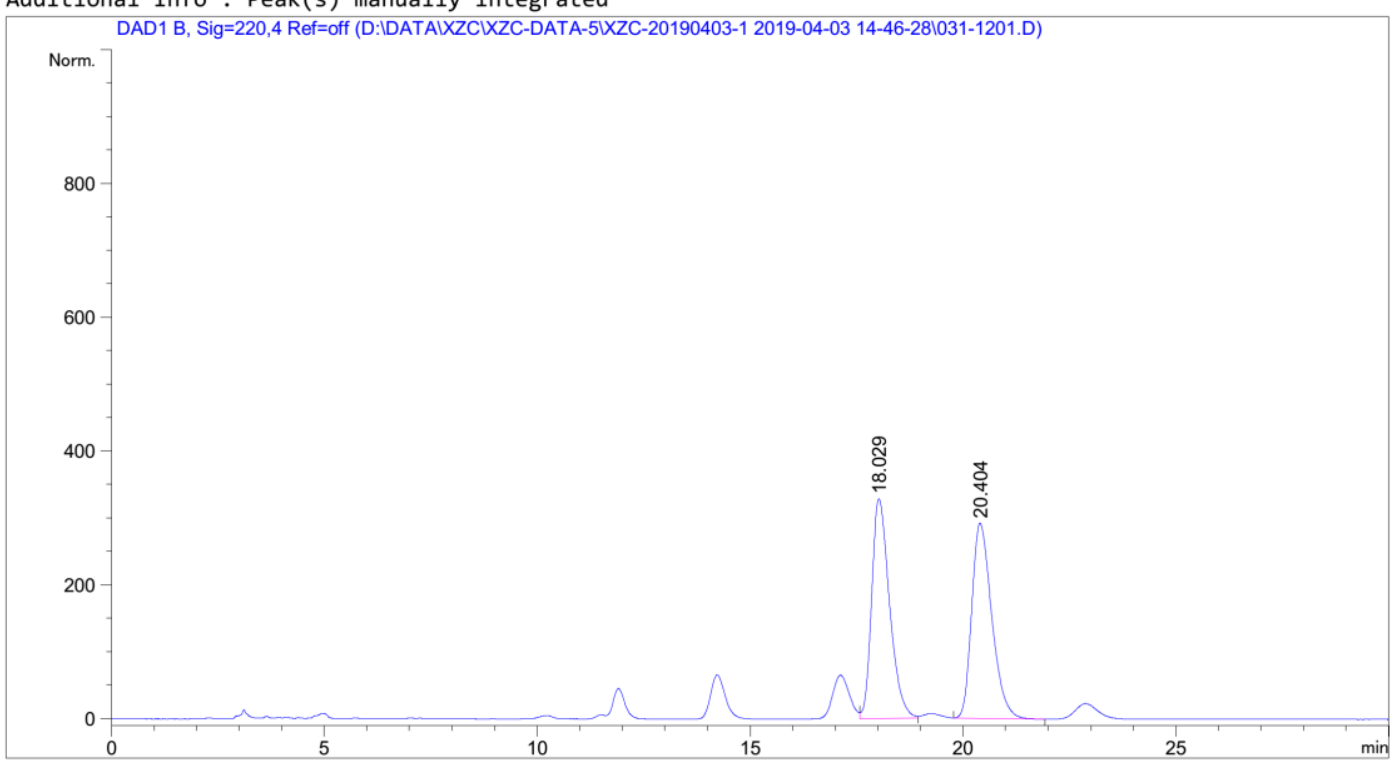

Area Percent Report

$\begin{array}{lll}================================= \\ \text { Sorted By } & : & \text { Signal } \\ \text { Multiplier } & : & 1.0000 \\ \text { Dilution } & : & 1.0000\end{array}$

Use Multiplier \& Dilution Factor with ISTDs

Signal 1: DAD1 B, Sig $=220,4$ Ref $=$ off

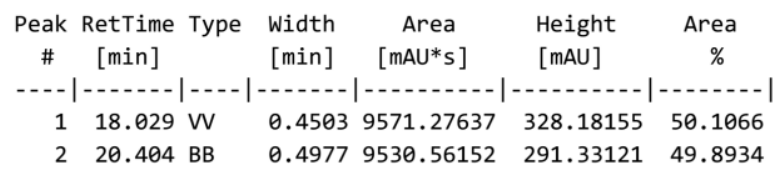

Totals :

$1.91018 \mathrm{e} 4 \quad 619.51276$ 
Data File D: \DATA \XZC \XZC-DATA-5\XZC-20190403-1 2019-04-03 14-46-28\032-1301.D Sample Name: LSL-ME

\begin{tabular}{|c|c|c|}
\hline $\begin{array}{l}\text { Acq. Operator } \\
\text { Acq. Instrument }\end{array}$ & $\begin{array}{l}\text { : } \\
: \text { Instrument } 2\end{array}$ & $\begin{array}{l}\text { Seq. Line : } 13 \\
\text { Location : Vial } 3\end{array}$ \\
\hline Injection Date & : 4/4/2019 1:30:03 AM & 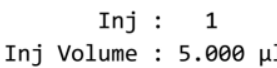 \\
\hline
\end{tabular}

Acq. Method : D: \DATA \XZC \XZC-DATA-5 \XZC-20190403-1 2019-04-03 14-46-28\DAD-OD(1-2)-98-2-

1ML-5UL-ALL-40MIN.M

: 9/21/2018 9:52:07 AM

Analysis Method : D: \METHOD\GUAN YUQING \DAD-OD(1-2)-96-4-0.5ML-5UL-ALL-120MIN.M

Last changed : 4/4/2019 8:57:46 AM

(modified after loading)

Additional Info : Peak(s) manually integrated

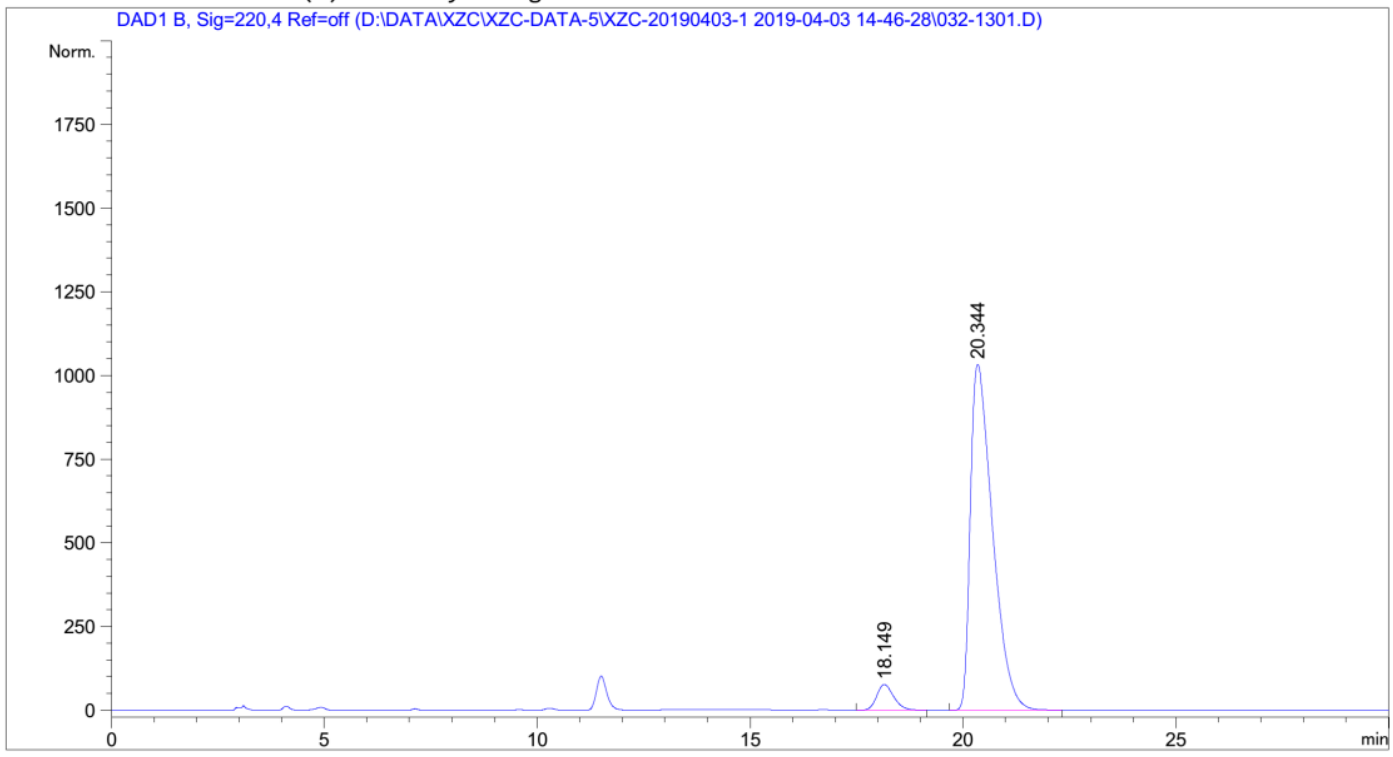

Area Percent Report

$\begin{array}{lll}================================ \\ \text { Sorted By } & : & \text { Signal } \\ \text { Multiplier } & : & 1.0000 \\ \text { Dilution } & : & 1.0000\end{array}$

Use Multiplier \& Dilution Factor with ISTDs

Signal 1: DAD1 B, Sig=220,4 Ref=off

\begin{tabular}{|c|c|c|c|c|c|}
\hline $\begin{array}{c}\text { Peak } \\
\#\end{array}$ & $\begin{array}{l}\text { RetTime Type } \\
\text { [min] }\end{array}$ & $\begin{array}{l}\text { Width } \\
\text { [min] }\end{array}$ & $\begin{array}{c}\text { Area } \\
{[\mathrm{mAU} * \mathrm{~s}]}\end{array}$ & $\begin{array}{l}\text { Height } \\
\text { [mAU] }\end{array}$ & $\begin{array}{c}\text { Area } \\
\%\end{array}$ \\
\hline$\cdots$ & $|-\ldots-n|--$ & & | & | & (-... \\
\hline 1 & 18.149 ВВ & 0.4229 & 2145.14160 & 76.56874 & 5.4264 \\
\hline 2 & 20.344 BB & 0.5441 & $3.73862 \mathrm{e} 4$ & 1033.43945 & 94.5736 \\
\hline Tota. & 1s: & & $3.95314 \mathrm{e} 4$ & 1110.00819 & \\
\hline
\end{tabular}


<smiles>CCOC(=O)C(CC=O)Cc1ccccc1</smiles>

Data File D: \DATA\LGY\LGY-3-140\LGY-3-140 2019-04-17 08-20-32\ө67-4201.D Sample Name: LSL-iPr rac

\begin{tabular}{|c|c|c|}
\hline Acq. Operator & $:$ & Seq. Line : 42 \\
\hline Acq. Instrument & : Instrument 2 & Location : Vial 67 \\
\hline Injection Date & : 4/18/2019 4:34:46 AM & $\begin{array}{rc}\text { Inj }: & 1 \\
\text { Inj Volume } & : 5.000 \mu\end{array}$ \\
\hline
\end{tabular}

Acq. Method : D: \DATA \LGY \LGY-3-140\LGY-3-140 2019-04-17 08-20-32\DAD-0J(1-6)-98-2-1ML3UL-ALL-60MIN.M

Last changed : 9/28/2018 8:55:24 AM

Analysis Method : D: \DATA \LGY \LGY-3-140\LGY-3-140 2019-04-17 08-20-32\DAD-0J(1-6)-98-2-1ML3UL-ALL-60MIN.M (Sequence Method)

Last changed : 4/18/2019 9:26:40 AM

(modified after loading)

Additional Info : Peak(s) manually integrated

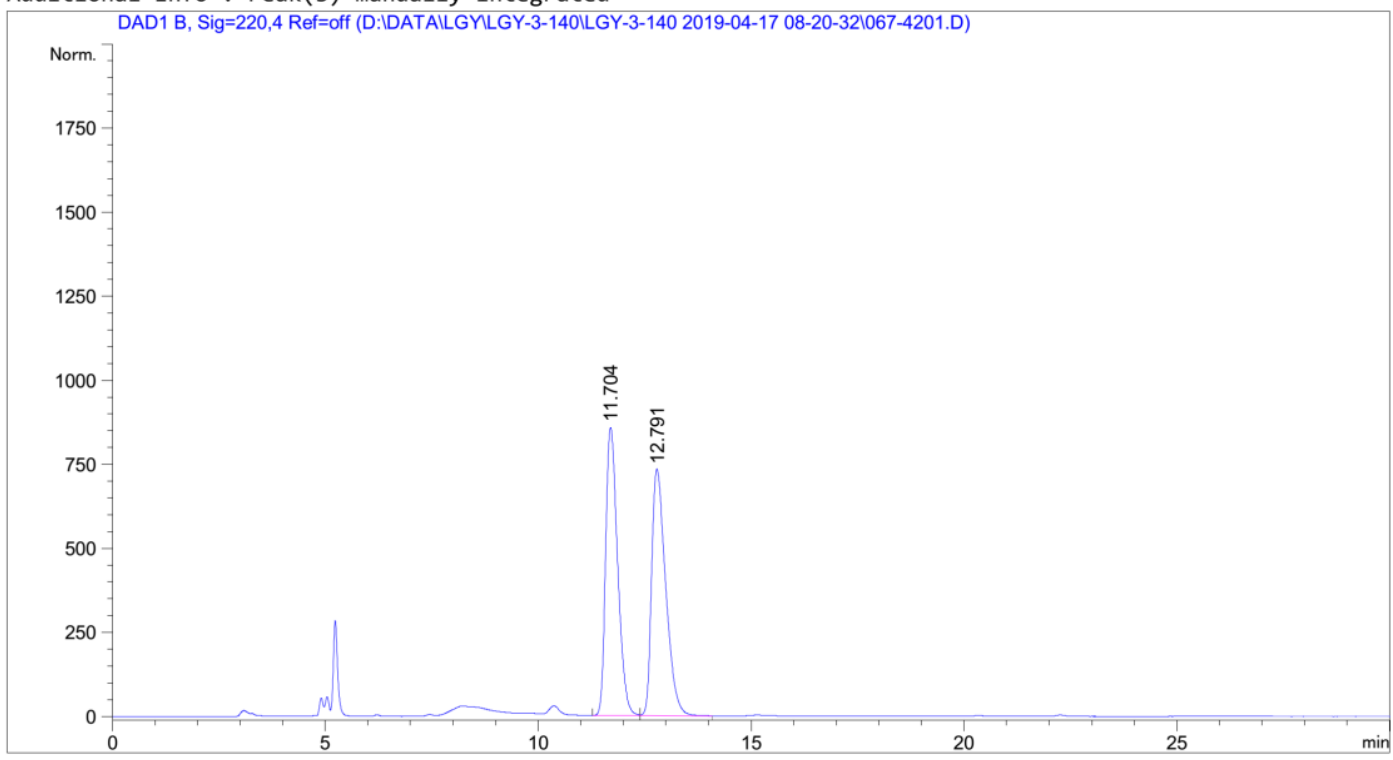

Area Percent Report

$\begin{array}{lll}=============================== \\ \text { Sorted By } & : & \text { Signal } \\ \text { Multiplier } & : & 1.0000 \\ \text { Dilution } & : & 1.0000\end{array}$

Use Multiplier \& Dilution Factor with ISTDs

Signal 1: DAD1 B, Sig=220,4 Ref=off

\begin{tabular}{|c|c|c|c|c|c|}
\hline $\begin{array}{c}\text { Peak } \\
\#\end{array}$ & $\begin{array}{l}\text { RetTime Type } \\
\text { [min] }\end{array}$ & $\begin{array}{l}\text { Width } \\
\text { [min] }\end{array}$ & $\begin{array}{c}\text { Area } \\
{\left[\mathrm{mAU}^{*} \mathrm{~s}\right]}\end{array}$ & $\begin{array}{l}\text { Height } \\
\text { [mAU] }\end{array}$ & $\begin{array}{c}\text { Area } \\
\%\end{array}$ \\
\hline & 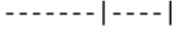 & & 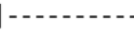 & 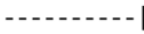 & - \\
\hline 1 & $11.704 \mathrm{BV}$ & 0.3019 & $1.67489 \mathrm{e} 4$ & 857.38049 & 49.8013 \\
\hline 2 & $12.791 \mathrm{VB}$ & 0.3484 & $1.68825 \mathrm{e} 4$ & 733.88715 & 50.1987 \\
\hline Tota & ; : & & $3.36314 \mathrm{e} 4$ & 1591.26764 & \\
\hline
\end{tabular}


Data File D: \DATA \LGY\LGY-3-140\LGY-3-140 2019-04-17 08-20-32\068-4301.D Sample Name: LSL-iPr

\begin{tabular}{|c|c|c|}
\hline $\begin{array}{l}\text { Acq. Operator } \\
\text { Acq. Instrument }\end{array}$ & : Instrument 2 & Seq. Line : 43 \\
\hline Injection Date & : 4/18/2019 5:35:47 AM & $\begin{array}{rc}\text { Inj : } & 1 \\
\text { Inj Volume } & 5\end{array}$ \\
\hline
\end{tabular}

Acq. Method : D: \DATA \LGY \LGY-3-140\LGY-3-140 2019-04-17 08-20-32 \DAD-0J(1-6)-98-2-1ML3UL-ALL-60MIN.M

Last changed : 9/28/2018 8:55:24 AM

Analysis Method : D: \DATA \LGY \LGY-3-140\LGY-3-140 2019-04-17 08-20-32\DAD-0J(1-6)-98-2-1ML3UL-ALL-60MIN.M (Sequence Method)

Last changed : 4/18/2019 9:29:19 AM (modified after loading)

Additional Info : Peak(s) manually integrated

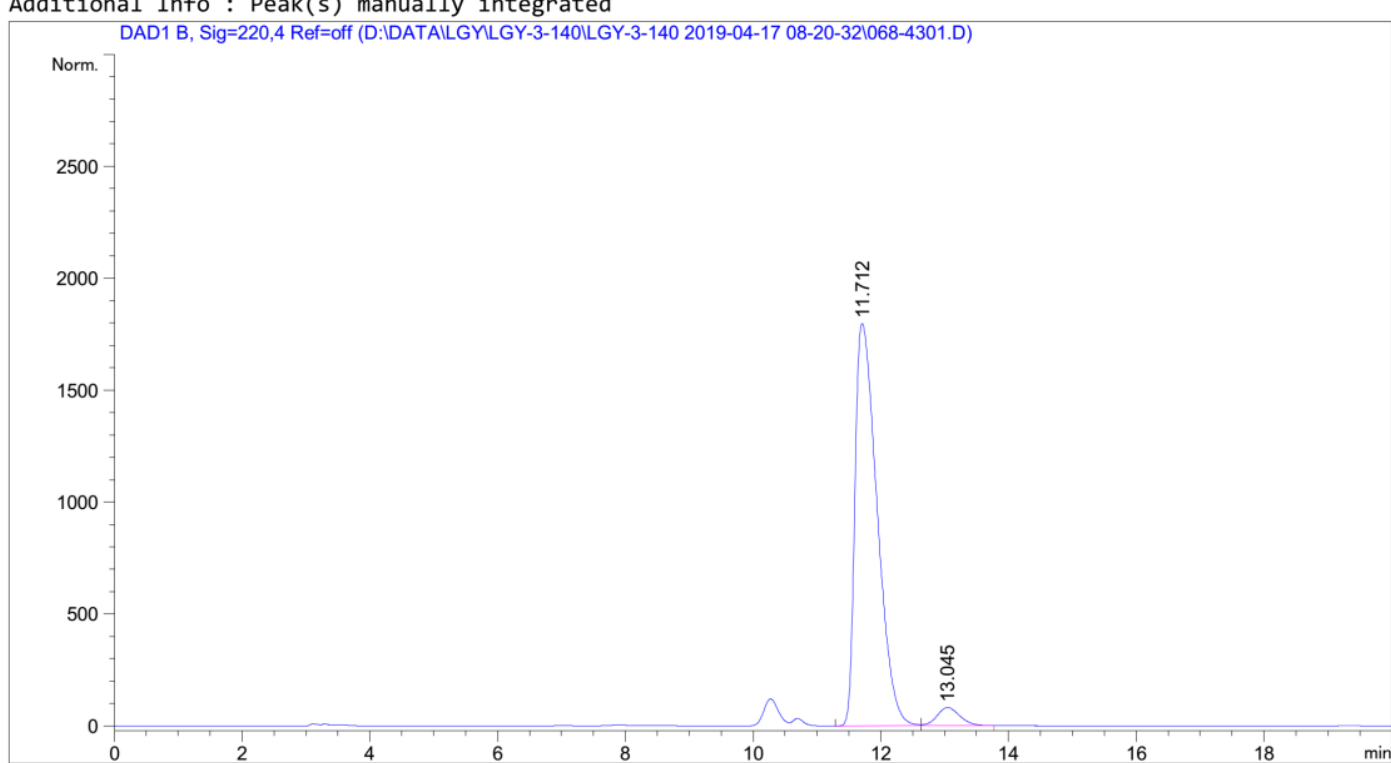

Area Percent Report

$\begin{array}{lll} & & \text { Area Percent } \\ ============================= \\ \text { Sorted By } & : & \text { Signal } \\ \text { Multiplier } & : & 1.0000 \\ \text { Dilution } & : & 1.0000\end{array}$

Use Multiplier \& Dilution Factor with ISTDs

Signal 1: DAD1 B, Sig $=220,4$ Ref $=0 f f$

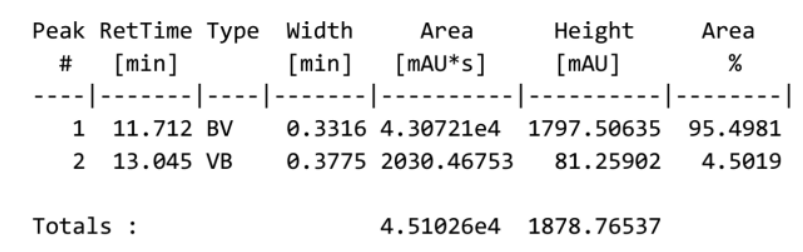


<smiles>O=CC[C@H](Cc1ccccc1)C(=O)OC(c1ccccc1)c1ccccc1</smiles>

Data File D: \DATA \LSL\LSL-4-69\LSL-4-69 2019-06-03 20-58-26\001-0301.D Sample Name: LSL-4-69-RAC

\begin{tabular}{|c|c|c|}
\hline Acq. Operator & : & Seq. Line : 3 \\
\hline Acq. Instrument & : Instrument 1 & Location : Vial 1 \\
\hline Injection Date & : 6/3/2019 9:21:08 PM & 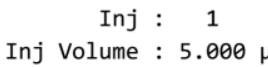 \\
\hline
\end{tabular}

Acq. Method : D: \DATA \LSL \LSL-4-69\LSL-4-69 2019-06-03 20-58-26\VWD-0D(1-2)-98-2-1ML-5UL220NM-40MIN.M

Last changed : 6/3/2019 8:56:27 PM

Analysis Method: D: \METHOD \LSL \VWD-OD(1-2)-98-2-1ML-5UL-220NM-40MIN.M

Last changed : 6/4/2019 8:43:57 AM

(modified after loading)

Additional Info : Peak(s) manually integrated

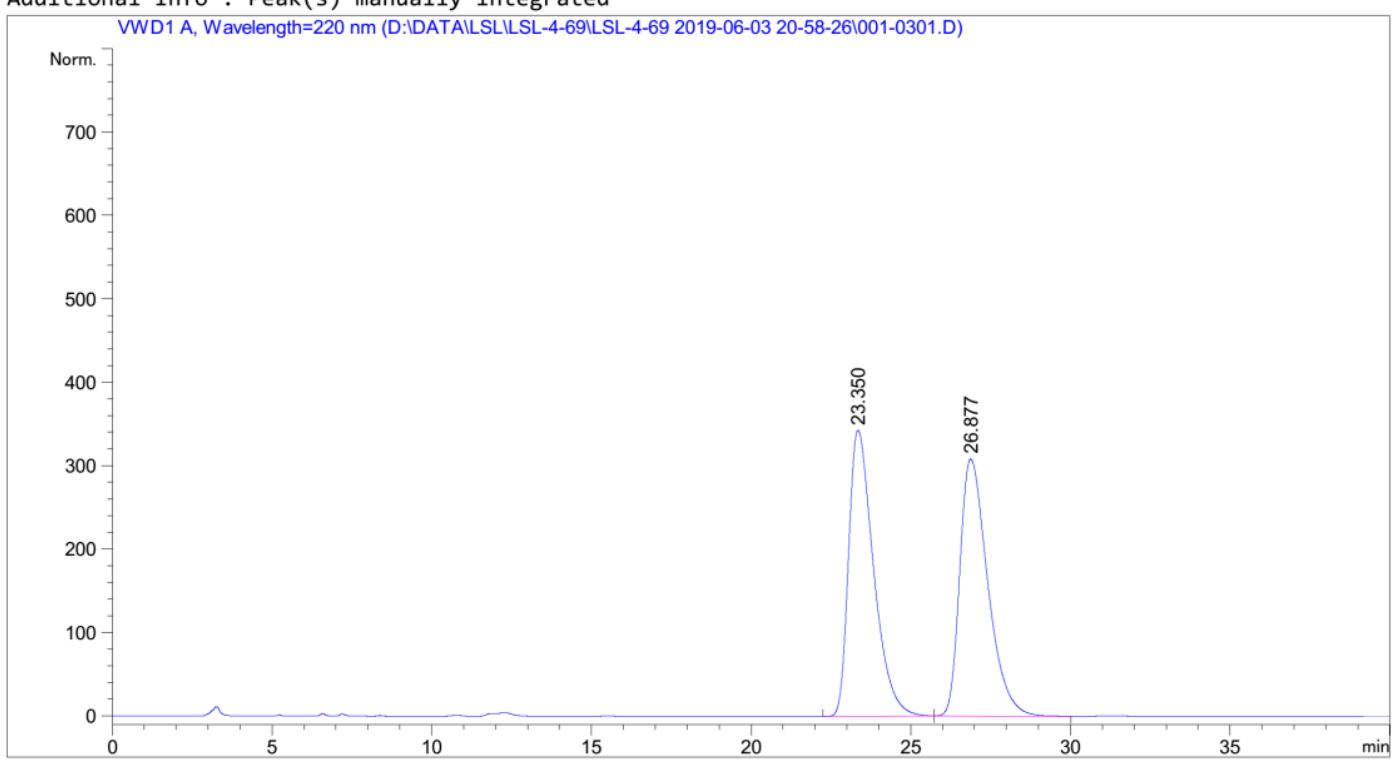

Area Percent Report

$\begin{array}{lll}================================= \\ \text { Sorted By } & : & \text { Signal } \\ \text { Multiplier } & : & 1.0000 \\ \text { Dilution } & : & 1.0000\end{array}$

Use Multiplier \& Dilution Factor with ISTDs

Signal 1: VWD1 A, Wavelength $=220 \mathrm{~nm}$

\begin{tabular}{|c|c|c|c|c|c|c|}
\hline $\begin{array}{c}\text { Peak } \\
\#\end{array}$ & $\begin{array}{c}\text { RetTime } \\
\text { [min] }\end{array}$ & Type & $\begin{array}{l}\text { Width } \\
\text { [min] }\end{array}$ & $\begin{array}{c}\text { Area } \\
{\left[\mathrm{mAU}^{*} \mathrm{~s}\right]}\end{array}$ & $\begin{array}{l}\text { Height } \\
{[\mathrm{mAU}]}\end{array}$ & $\begin{array}{c}\text { Area } \\
\%\end{array}$ \\
\hline$-\ldots$ & - & & - & - & - & | \\
\hline 1 & 23.350 & BB & 0.8327 & $1.86351 \mathrm{e} 4$ & 343.05798 & 49.9112 \\
\hline 2 & 26.877 & BB & 0.9316 & $1.87014 \mathrm{e} 4$ & 307.89487 & 50.0888 \\
\hline Tota & : & & & $3.73364 \mathrm{e} 4$ & 650.95285 & \\
\hline
\end{tabular}


Data File D: \DATA \LSL\LSL-4-69\LSL-4-69 2019-06-03 20-58-26\002-0401.D Sample Name: LSL-4-69

$\begin{array}{llr}\text { Acq. Operator }: & \text { Seq. Line }: 4 \\ \text { Acq. Instrument : Instrument } 1 & \text { Location : Vial } 2 \\ \text { Injection Date }: 6 / 3 / 2019 \text { 10:01:58 PM } & \text { Inj : } 1\end{array}$

Acq. Method : D: \DATA \LSL \LSL-4-69\LSL-4-69 2019-06-03 20-58-26\VWD-OD(1-2)-98-2-1ML-5UL220NM-4OMIN.M

Last changed : 6/3/2019 8:56:27 PM

Analysis Method: D: \METHOD \LSL \VWD-OD(1-2)-98-2-1ML-5UL-220NM-40MIN.M

Last changed : 6/4/2019 8:45:14 AM

(modified after loading)

Additional Info : Peak(s) manually integrated

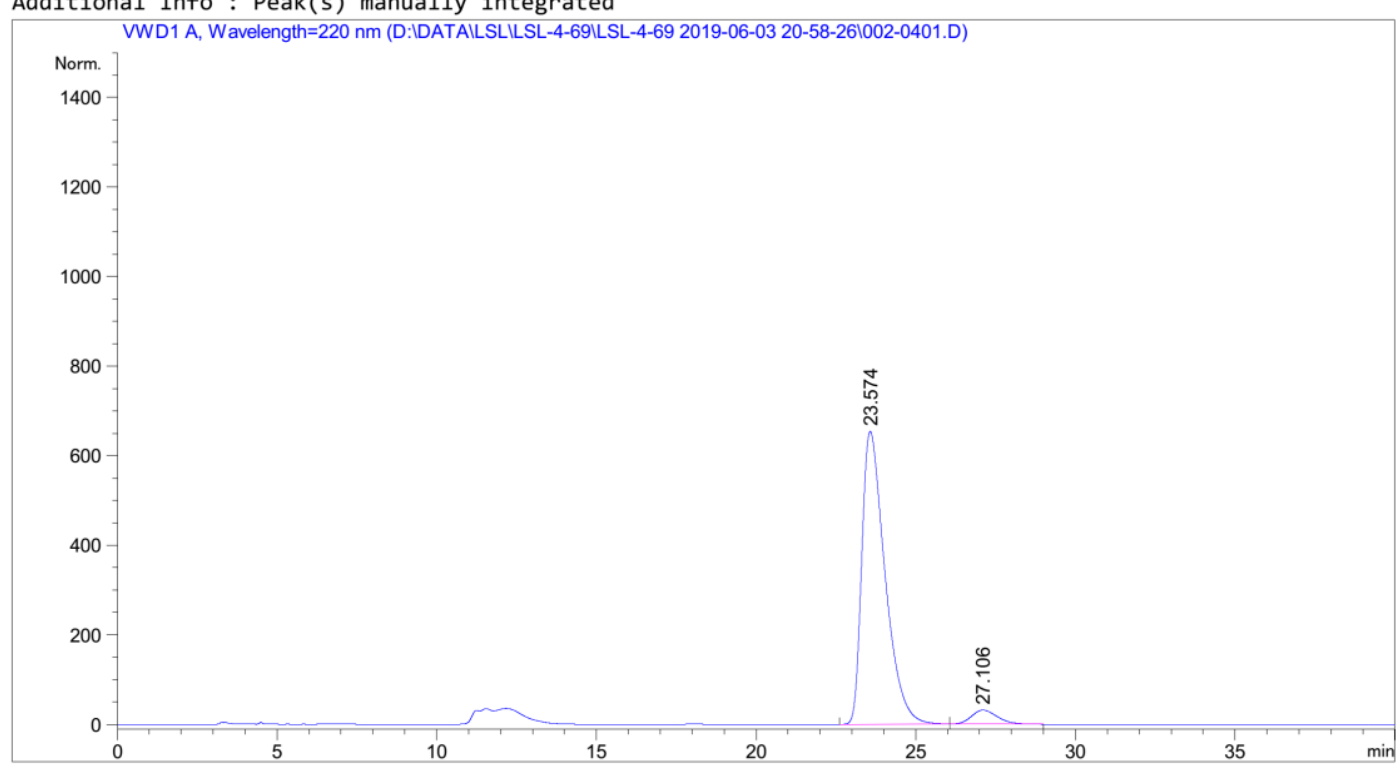

Area Percent Report

$\begin{array}{lll}\text { Sorted By } & : & \text { Signal } \\ \text { Multiplier } & : & 1.0000 \\ \text { Dilution } & : & 1.0000\end{array}$

Use Multiplier \& Dilution Factor with ISTDs

Signal 1: VWD1 A, Wavelength $=220 \mathrm{~nm}$

\begin{tabular}{|c|c|c|c|c|c|}
\hline $\begin{array}{c}\text { Peak } \\
\#\end{array}$ & $\begin{array}{l}\text { RetTime Type } \\
\text { [min] }\end{array}$ & $\begin{array}{l}\text { Width } \\
\text { [min] }\end{array}$ & $\begin{array}{c}\text { Area } \\
{\left[\mathrm{mAU}^{*} \mathrm{~s}\right]}\end{array}$ & $\begin{array}{l}\text { Height } \\
{[\mathrm{mAU}]}\end{array}$ & $\begin{array}{c}\text { Area } \\
\%\end{array}$ \\
\hline$\ldots$ & $-\ldots$ & . & |-... & | & | \\
\hline 1 & $23.574 \mathrm{BB}$ & 0.7639 & $3.29479 \mathrm{e} 4$ & 653.87494 & 94.9983 \\
\hline 2 & 27.106 BВ & 0.8551 & 1734.73364 & 31.12970 & 5.0017 \\
\hline Tota & : & & $3.46826 \mathrm{e} 4$ & 685.00464 & \\
\hline
\end{tabular}


<smiles>O=CCC(Cc1ccccc1)C(=O)Oc1ccccc1</smiles>

Data File D: \DATA \XZC\XZC-DATA-5\XZC-20190403-1 2019-04-03 14-46-28\033-1401.D Sample Name: LSL-OPH RAC

\begin{tabular}{|c|c|c|}
\hline Acq. Operator & $:$ & Seq. Line : 14 \\
\hline Acq. Instrument & : Instrument 2 & Location : Vial 33 \\
\hline Injection Date & : 4/4/2019 2:11:04 AM & 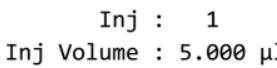 \\
\hline
\end{tabular}

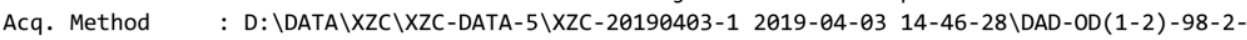
1ML-5UL-ALL-40MIN.M

Last changed : 9/21/2018 9:52:07 AM

Analysis Method : D: \METHOD\GUAN YUQING \DAD-OD(1-2)-96-4-0.5ML-5UL-ALL-120MIN.M

Last changed : 4/4/2019 8:59:30 AM

(modified after loading)

Additional Info : Peak(s) manually integrated

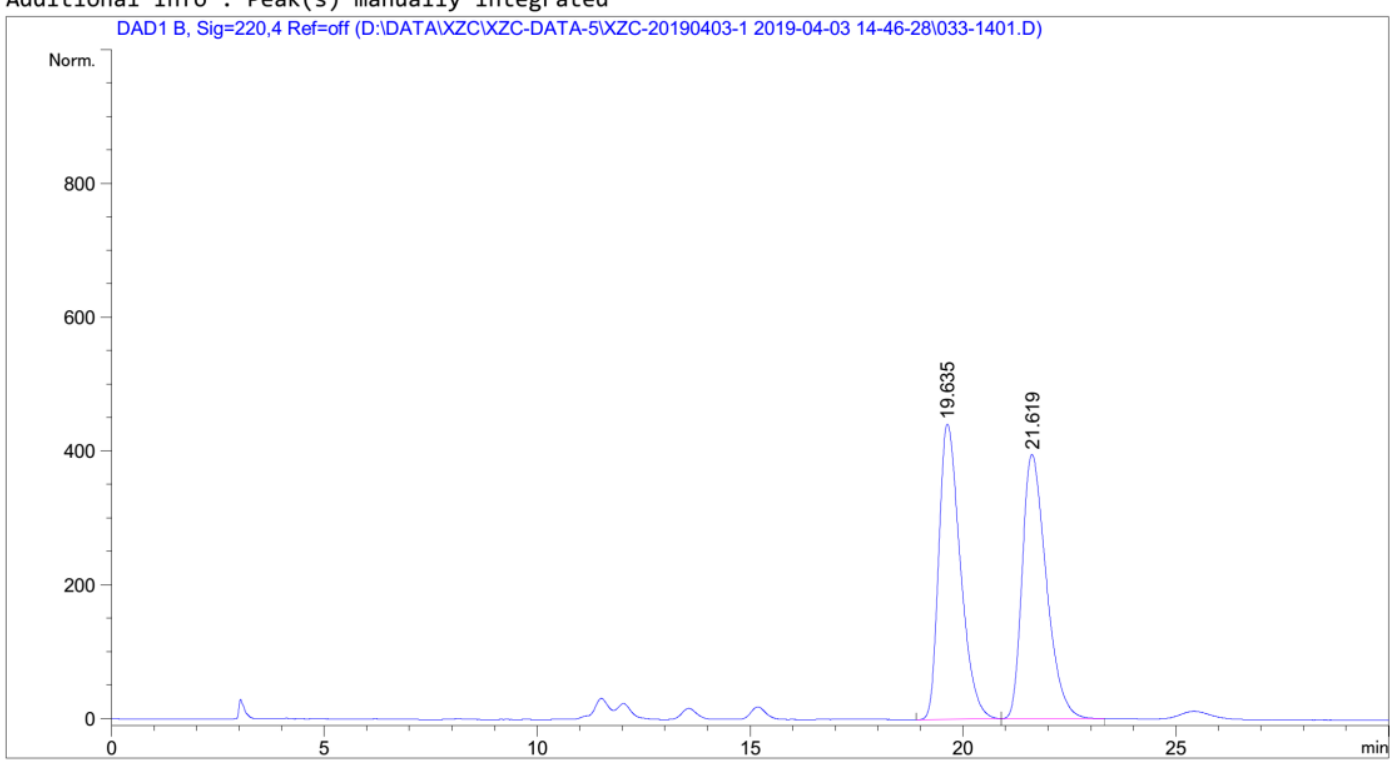

Area Percent Report

$\begin{array}{lll}================================ \\ \text { Sorted By } & : & \text { Signal } \\ \text { Multiplier } & : & 1.0000 \\ \text { Dilution } & : & 1.0000\end{array}$

Use Multiplier \& Dilution Factor with ISTDs

Signal 1: DAD1 B, Sig $=220,4$ Ref $=$ off

\begin{tabular}{|c|c|c|c|c|c|c|}
\hline $\begin{array}{c}\text { Peak } \\
\#\end{array}$ & $\begin{array}{c}\text { RetTime } \\
\text { [min] }\end{array}$ & Type & $\begin{array}{l}\text { Width } \\
\text { [min] }\end{array}$ & $\begin{array}{c}\text { Area } \\
{\left[\mathrm{mAU}^{*} \mathrm{~s}\right]}\end{array}$ & $\begin{array}{l}\text { Height } \\
{[\mathrm{mAU}]}\end{array}$ & $\begin{array}{c}\text { Area } \\
\%\end{array}$ \\
\hline -... & - & & 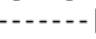 & | & | & | \\
\hline 1 & 19.635 & BB & 0.5400 & $1.55139 \mathrm{e} 4$ & 441.40121 & 49.9944 \\
\hline 2 & 21.619 & BB & 0.5984 & $1.55174 \mathrm{e} 4$ & 394.91980 & 50.0056 \\
\hline Tota & s : & & & $3.10313 \mathrm{e} 4$ & 836.32101 & \\
\hline
\end{tabular}


Data File D: \DATA \XZC \XZC-DATA-5\XZC-20190403-1 2019-04-03 14-46-28\034-1501.D Sample Name: LSL-OPH

\begin{tabular}{|c|c|c|}
\hline Acq. Operator & $:$ & Seq. Line : 15 \\
\hline Acq. Instrument & : Instrument 2 & Location : Vial 34 \\
\hline Injection Date & : 4/4/2019 2:52:05 AM & 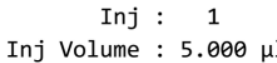 \\
\hline
\end{tabular}

Acq. Method : D: \DATA \XZC \XZC-DATA-5 \XZC-20190403-1 2019-04-03 14-46-28\DAD-OD(1-2)-98-2-

1ML-5UL-ALL-40MIN.M

: 9/21/2018 9:52:07 AM

Analysis Method : D: \METHOD\GUAN YUQING \DAD-OD(1-2)-96-4-0.5ML-5UL-ALL-120MIN.M

Last changed : 4/4/2019 8:59:30 AM

(modified after loading)

Additional Info : Peak(s) manually integrated

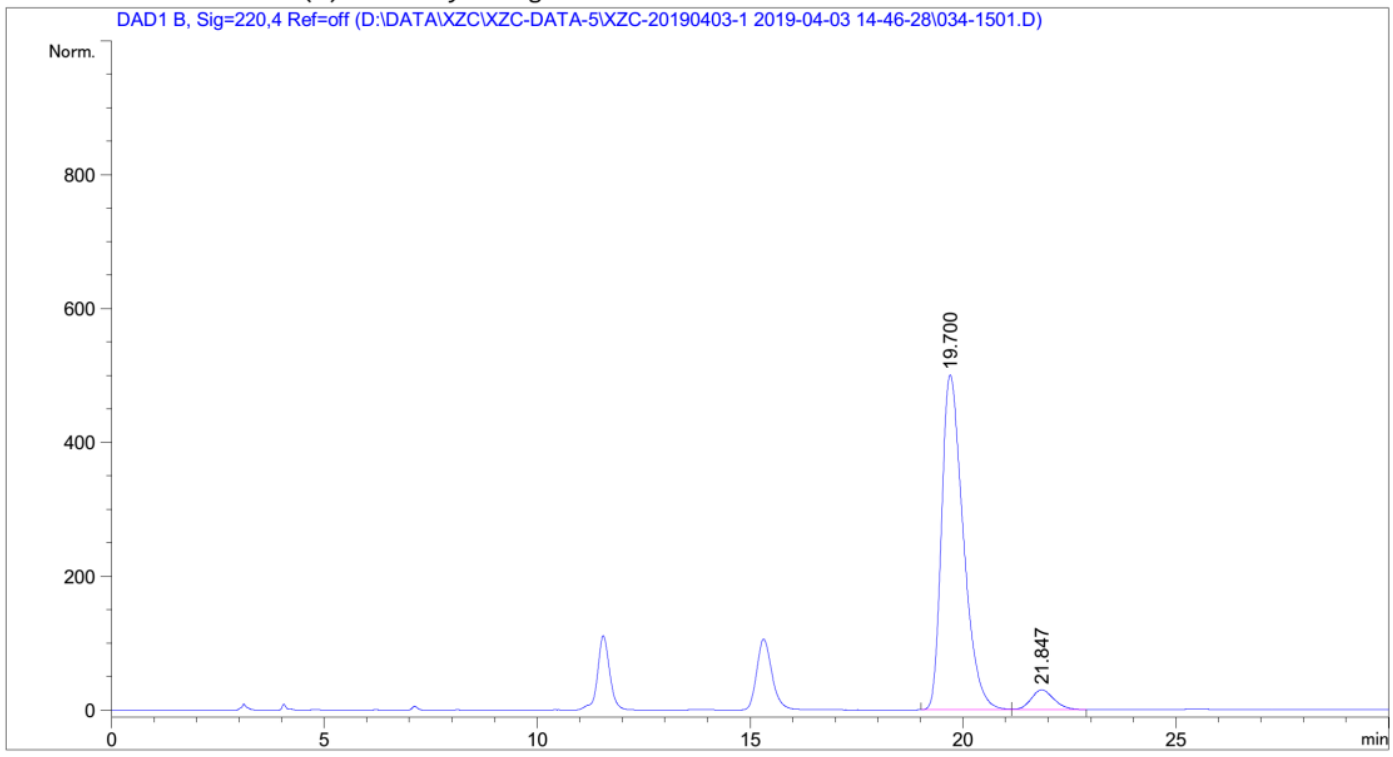

Area Percent Report

$\begin{array}{lll}================================ \\ \text { Sorted By } & : & \text { Signal } \\ \text { Multiplier } & : & 1.0000 \\ \text { Dilution } & : & 1.0000\end{array}$

Use Multiplier \& Dilution Factor with ISTDs

Signal 1: DAD1 B, Sig=220,4 Ref=off

\begin{tabular}{|c|c|c|c|c|c|}
\hline $\begin{array}{c}\text { Peak } \\
\#\end{array}$ & $\begin{array}{l}\text { RetTime Type } \\
\text { [min] }\end{array}$ & $\begin{array}{l}\text { Width } \\
\text { [min] }\end{array}$ & $\begin{array}{c}\text { Area } \\
{\left[\mathrm{mAU}^{*} \mathrm{~s}\right]}\end{array}$ & $\begin{array}{l}\text { Height } \\
\text { [mAU] }\end{array}$ & $\begin{array}{c}\text { Area } \\
\%\end{array}$ \\
\hline & $-0 .-1$ & & |-- & $-\ldots$ & - - - - - \\
\hline 1 & 19.700 BV & 0.5455 & $1.77180 \mathrm{e} 4$ & 499.95978 & 94.1148 \\
\hline 2 & 21.847 VB & 0.5168 & 1107.94885 & 29.45843 & 5.8852 \\
\hline Total & & & $1.88259 \mathrm{e} 4$ & 529.41820 & \\
\hline
\end{tabular}


<smiles>O=CCC(Cc1ccccc1)C(=O)OCc1ccccc1</smiles>

Data File D: \DATA \XZC\XZC-DATA-5\XZC-20190403-1 2019-04-03 14-46-28\035-1601.D Sample Name: LSL-OBN RAC

\begin{tabular}{|c|c|c|}
\hline Acq. Operator & : & Seq. Line : 16 \\
\hline Acq. Instrument & : Instrument 2 & Location : Vial 35 \\
\hline Injection Date & : 4/4/2019 3:33:05 AM & $\begin{array}{rlc}\text { Inj } & : & 1 \\
\text { Inj Volume } & : & 5.000 \mu\end{array}$ \\
\hline
\end{tabular}

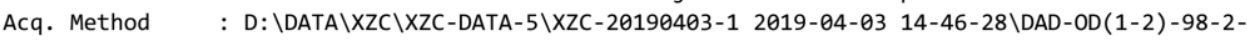
1ML-5UL-ALL-40MIN.M

Last changed : 9/21/2018 9:52:07 AM

Analysis Method : D: \METHOD\GUAN YUQING \DAD-OD(1-2)-96-4-0.5ML-5UL-ALL-120MIN.M

Last changed : 4/4/2019 9:03:32 AM

(modified after loading)

Additional Info: $\operatorname{Peak}(\mathrm{s})$ manually integrated

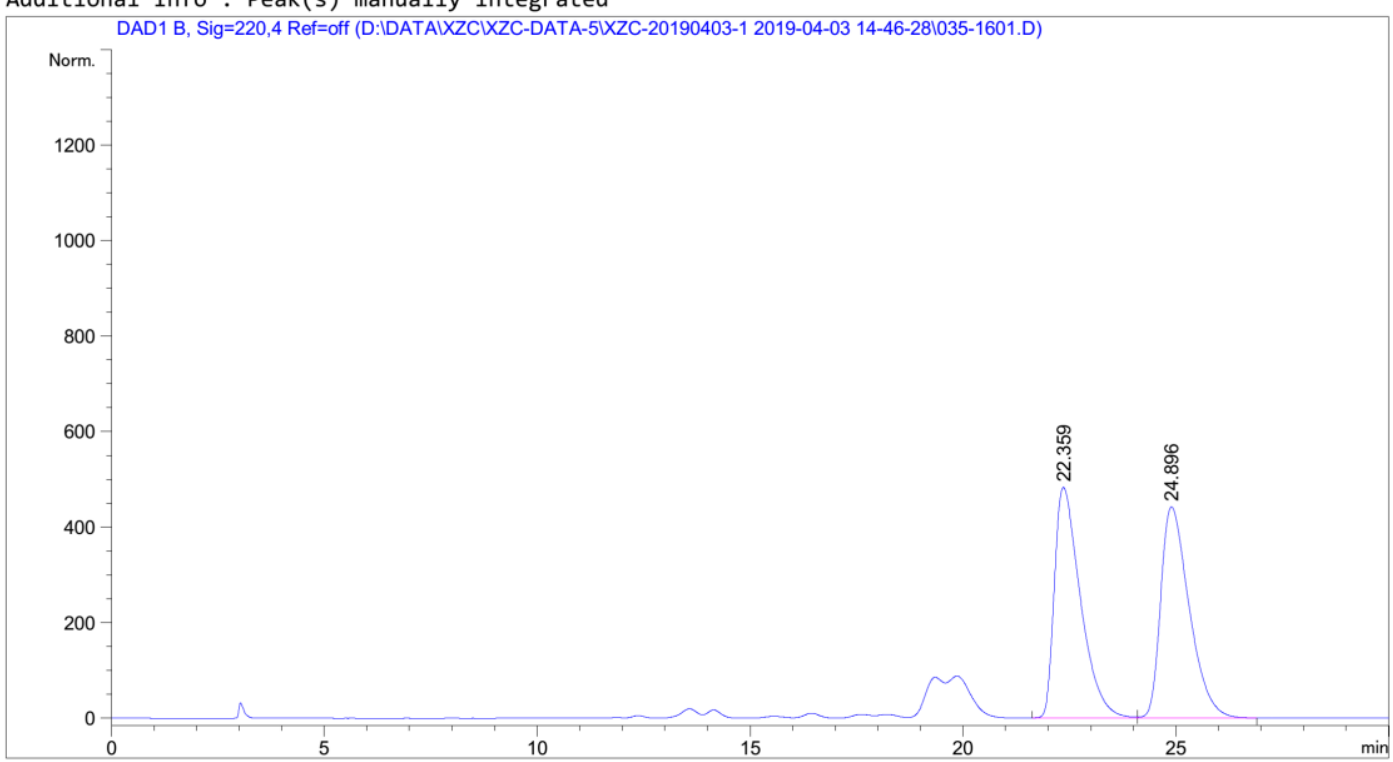

Area Percent Report

$\begin{array}{lll}================================ \\ \text { Sorted By } & : & \text { Signal } \\ \text { Multiplier } & : & 1.0000 \\ \text { Dilution } & : & 1.0000\end{array}$

Use Multiplier \& Dilution Factor with ISTDs

Signal 1: DAD1 B, Sig $=220,4$ Ref $=$ off

\begin{tabular}{cccccc}
$\begin{array}{c}\text { Peak RetTime Type } \\
\text { W }\end{array}$ [min] & $\begin{array}{c}\text { Width } \\
\text { [min] }\end{array}$ & $\begin{array}{c}\text { Area } \\
{[\text { mAU*s] }}\end{array}$ & $\begin{array}{l}\text { Height } \\
{[\mathrm{mAU}]}\end{array}$ & $\begin{array}{c}\text { Area } \\
\%\end{array}$ \\
\hline 1 & 22.359 BV & 0.6387 & $2.05395 \mathrm{e} 4$ & 482.44583 & 50.0099 \\
2 & 24.896 VB & 0.6936 & $2.05314 \mathrm{e} 4$ & 442.30383 & 49.9901
\end{tabular}

Totals : $\quad 4.10709 \mathrm{e} 4 \quad 924.74966$ 
Data File D: \DATA \XZC \XZC-DATA-5 \XZC-20190403-1 2019-04-03 14-46-28\036-1701.D Sample Name: LSL-OBN

\begin{tabular}{|c|c|c|}
\hline $\begin{array}{l}\text { Acq. Operator } \\
\text { Acq. Instrument }\end{array}$ & : & Seq. Line : 17 \\
\hline Injection Date & : 4/4/2019 4:14:07 AM & $\begin{array}{rcc}\text { Inj }: & 1 \\
\text { Inj Volume } & : & 5.000\end{array}$ \\
\hline
\end{tabular}

Acq. Method : D: \DATA \XZC \XZC-DATA-5 \XZC-20190403-1 2019-04-03 14-46-28\DAD-OD(1-2)-98-2-

1ML-5UL-ALL-40MIN.M

: 9/21/2018 9:52:07 AM

Analysis Method: D: \METHOD\GUAN YUQING \DAD-OD(1-2)-96-4-0.5ML-5UL-ALL-120MIN.M

Last changed : 4/4/2019 9:04:57 AM

(modified after loading)

Additional Info : Peak(s) manually integrated

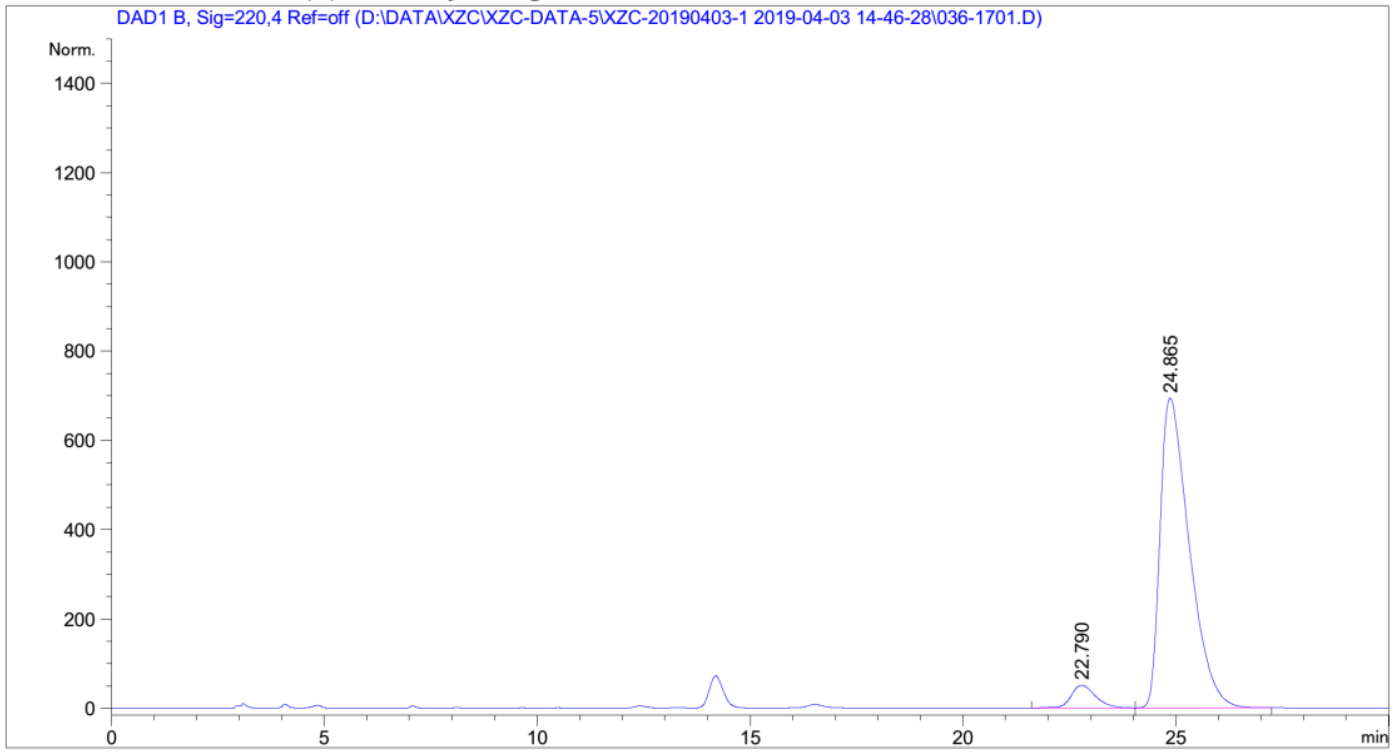

Area Percent Report

$\begin{array}{lll}============================= \\ \text { Sorted By } & : & \text { Signal } \\ \text { Multiplier } & : & 1.0000 \\ \text { Dilution } & : & 1.0000\end{array}$

Use Multiplier \& Dilution Factor with ISTDs

Signal 1: DAD1 B, Sig=220,4 Ref=off

\begin{tabular}{|c|c|c|c|c|c|}
\hline $\begin{array}{c}\text { Peak } \\
\#\end{array}$ & $\begin{array}{l}\text { RetTime Type } \\
\text { [min] }\end{array}$ & $\begin{array}{l}\text { Width } \\
\text { [min] }\end{array}$ & $\begin{array}{c}\text { Area } \\
{\left[\mathrm{mAU}^{*} \mathrm{~s}\right]}\end{array}$ & $\begin{array}{l}\text { Height } \\
{[\mathrm{mAU}]}\end{array}$ & $\begin{array}{c}\text { Area } \\
\%\end{array}$ \\
\hline$\ldots$ & - & & | & | & | \\
\hline 1 & $22.790 \mathrm{BB}$ & 0.5667 & 2054.15601 & 51.13189 & 5.8400 \\
\hline 2 & 24.865 BB & 0.6944 & 3.31199e4 & 694.43408 & 94.1600 \\
\hline \multicolumn{3}{|c|}{ Totals : } & $3.51741 \mathrm{e} 4$ & 745.56597 & \\
\hline
\end{tabular}


<smiles>CCNC(=O)C(CC=O)Cc1ccccc1</smiles>

Data File D: \DATA\LSL\LSL-4-31\LSL-4-31-1 2019-04-15 10-05-12\011-0301.D Sample Name: LSL-4-21-1

\begin{tabular}{|c|c|c|}
\hline Acq. Operator & : & Seq. Line : \\
\hline Acq. Instrument & : Instrument 2 & Location : Vial 11 \\
\hline Injection Date & : 4/15/2019 10:30:20 AM & 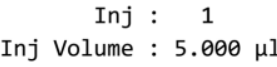 \\
\hline
\end{tabular}

Acq. Method : D: \DATA \LSL\LSL-4-31\LSL-4-31-1 2019-04-15 10-05-12 \DAD-OD(1-2)-95-5-1ML5UL-ALL-30MIN.M

Last changed : 4/15/2019 10:17:23 AM

Analysis Method: D: \METHOD \LG \DAD-OD(1-2)-80-20-1ML-5UL-ALL-60MIN.M

Last changed : 9/8/2019 10:54:01 AM

(modified after loading)

Additional Info : Peak(s) manually integrated

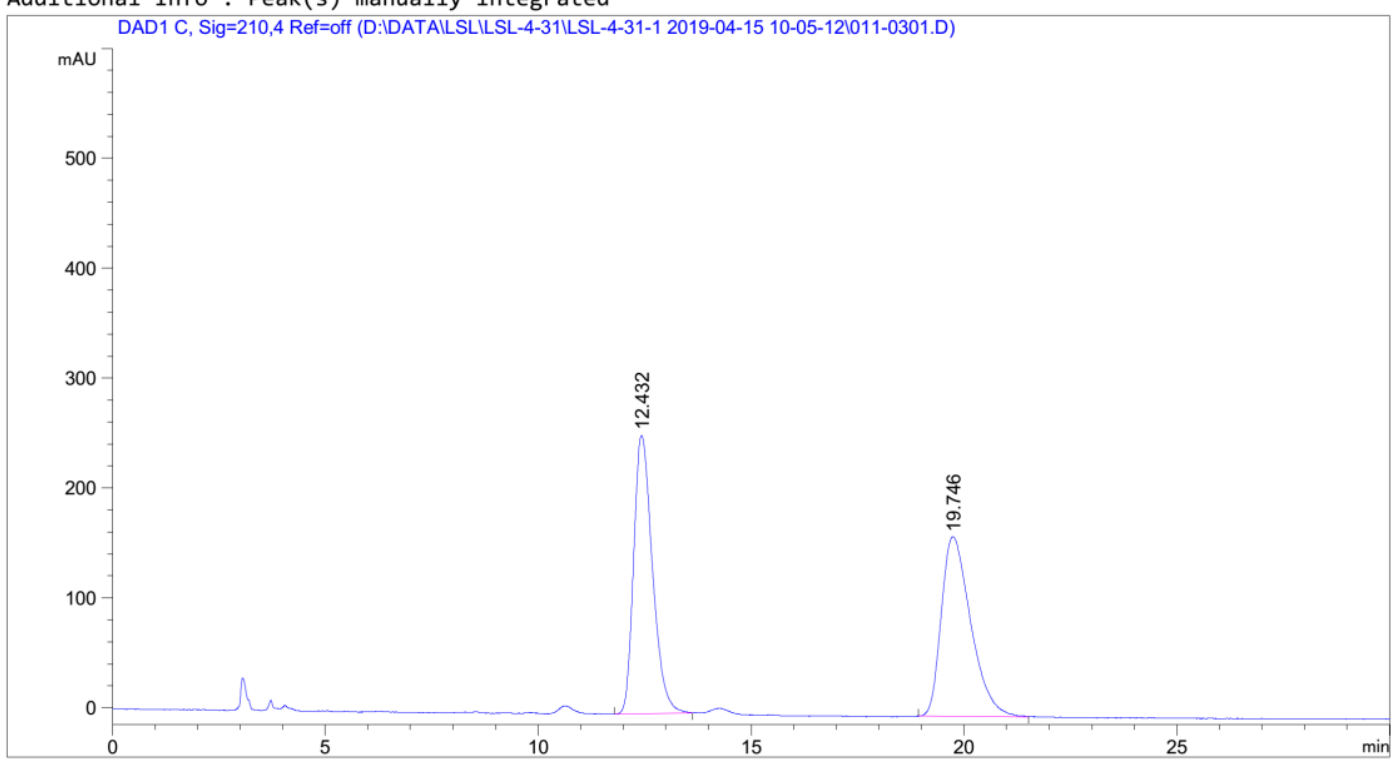

Area Percent Report

$\begin{array}{lll}================================ \\ \text { Sorted By } & : & \text { Signal } \\ \text { Multiplier } & : & 1.0000 \\ \text { Dilution } & : & 1.0000\end{array}$

Use Multiplier \& Dilution Factor with ISTDs

Signal 1: DAD1 C, Sig $=210,4$ Ref $=$ off

\begin{tabular}{cccccc}
$\begin{array}{c}\text { Peak RetTime Type } \\
\text { Width }\end{array}$ [min] & $\begin{array}{c}\text { Area } \\
{[\mathrm{min}]}\end{array}[\mathrm{mAU}$ ] $]$ & $\begin{array}{c}\text { Height } \\
{[\mathrm{mAU}]}\end{array}$ & $\begin{array}{c}\text { Area } \\
\%\end{array}$ \\
\hline 1 & 12.432 BV & 0.4519 & 7775.30762 & 253.46440 & 49.7057 \\
2 & 19.746 BV & 0.6475 & 7867.37207 & 163.46220 & 50.2943
\end{tabular}

Totals :

$1.56427 \mathrm{e} 4 \quad 416.92661$ 
Data File D: \DATA \LSL\LSL-4-31\LSL-4-31-1 2019-04-15 10-05-12\012-0401.D Sample Name: LSL-4-31-1

\begin{tabular}{|c|c|c|}
\hline Acq. Operator & : & Seq. Line : 4 \\
\hline Acq. Instrument & : Instrument 2 & Location : Vial 12 \\
\hline Injection Date & : 4/15/2019 11:01:19 AM & $\begin{array}{rlc}\text { Inj } & : & 1 \\
\text { Inj Volume } & : & 5.000\end{array}$ \\
\hline
\end{tabular}

Acq. Method : D: \DATA \LSL \LSL-4-31\LSL-4-31-1 2019-04-15 10-05-12 \DAD-OD(1-2)-95-5-1ML-

$\begin{aligned} & \text { 5UL-ALL-30MIN.M } \\ \text { Last changed } & \text { 4/15/2019 10:17:23 AM }\end{aligned}$

Analysis Method : D: \METHOD \LG\DAD-OD(1-2)-80-20-1ML-5UL-ALL-60MIN.M

Last changed : 9/8/2019 10:52:16 AM

(modified after loading)

Additional Info : Peak(s) manually integrated

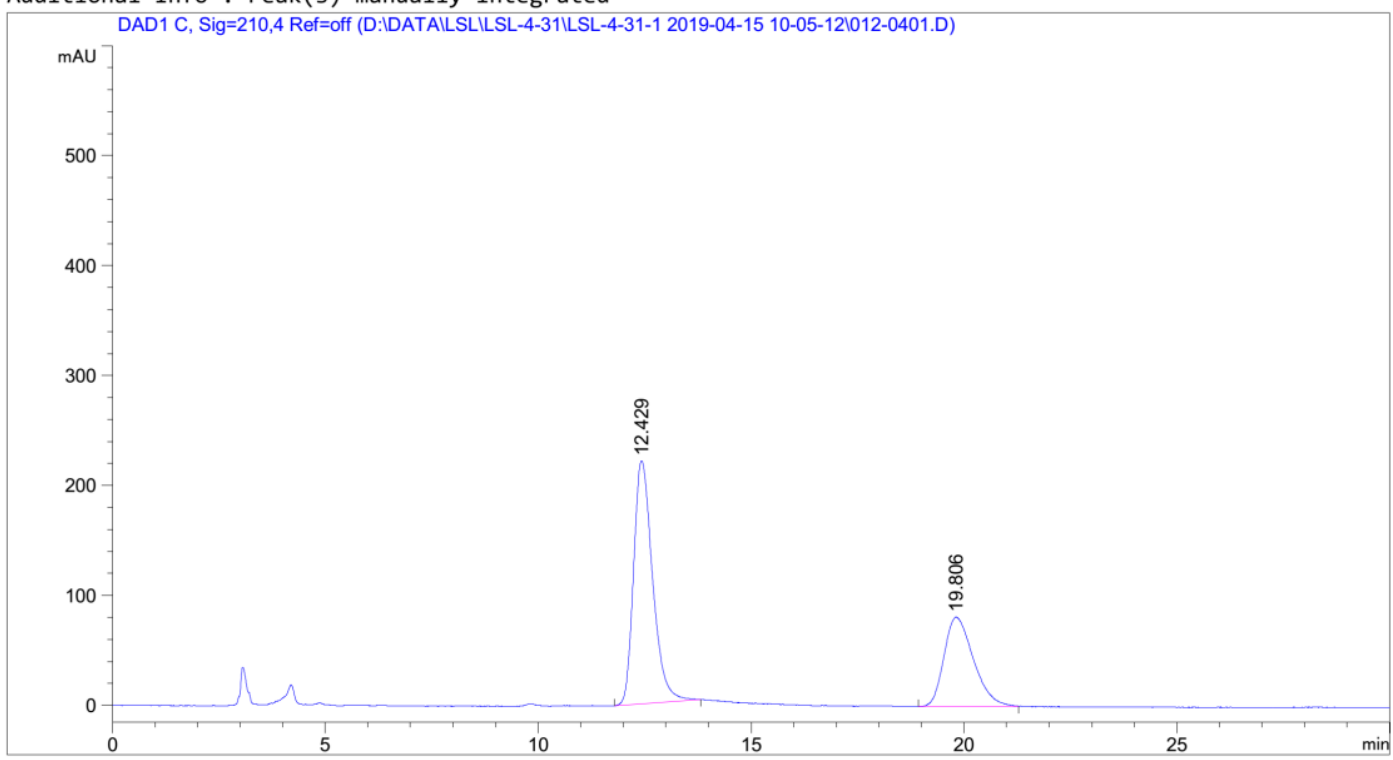

Area Percent Report

$\begin{array}{lll}================================+ \\ \text { Sorted By } & : & \text { Signal } \\ \text { Multiplier } & : & 1.0000 \\ \text { Dilution } & : & 1.0000\end{array}$

Use Multiplier \& Dilution Factor with ISTDs

Signal 1: DAD1 C, Sig=210,4 Ref=off

\begin{tabular}{|c|c|c|c|c|c|}
\hline $\begin{array}{c}\text { Peak } \\
\#\end{array}$ & $\begin{array}{l}\text { RetTime Type } \\
\text { [min] }\end{array}$ & $\begin{array}{l}\text { Width } \\
\text { [min] }\end{array}$ & $\begin{array}{c}\text { Area } \\
{[\mathrm{mAU} * \mathrm{~s}]}\end{array}$ & $\begin{array}{l}\text { Height } \\
\text { [mAU] }\end{array}$ & $\begin{array}{c}\text { Area } \\
\%\end{array}$ \\
\hline & $\mid$ & & | & - & - - - - - \\
\hline 1 & $12.429 \mathrm{BB}$ & 0.4564 & 6871.85010 & 221.21272 & 63.8419 \\
\hline 2 & 19.806 BV & 0.5887 & 3892.00366 & 81.26534 & 36.1581 \\
\hline Tota. & & & $1.07639 \mathrm{e} 4$ & 302.47806 & \\
\hline
\end{tabular}




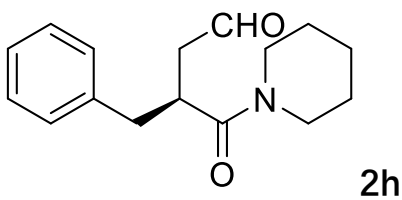

Data File D: \DATA\GUAN YUQING\LK-A\LKB-190415 2019-04-15 08-56-25\041-0701.D Sample Name: LSL-4-21-3

\begin{tabular}{|c|c|c|}
\hline Acq. Operator & : & Seq. Line : 7 \\
\hline Acq. Instrument & : Instrument 1 & Location : Vial 41 \\
\hline Injection Date & : 4/15/2019 1:01:13 PM & $\begin{array}{rlc}\text { Inj } & : & 1 \\
\text { Inj Volume } & : & 3.000 \mu \mathrm{l}\end{array}$ \\
\hline
\end{tabular}

Acq. Method : D: \DATA \GUAN YUQING \LK-A \LKB-190415 2019-04-15 08-56-25\VWD-AD(1-2)-95-51ML-3UL-220NM-40MIN.M

Last changed : 6/7/2018 11:25:33 AM

Analysis Method: D: \METHOD LG \VWD-AD(1-2)-80-20-1ML-3UL-210NM-60MIN.M

Last changed : 8/13/2019 5:09:43 PM

(modified after loading)

Additional Info : Peak(s) manually integrated

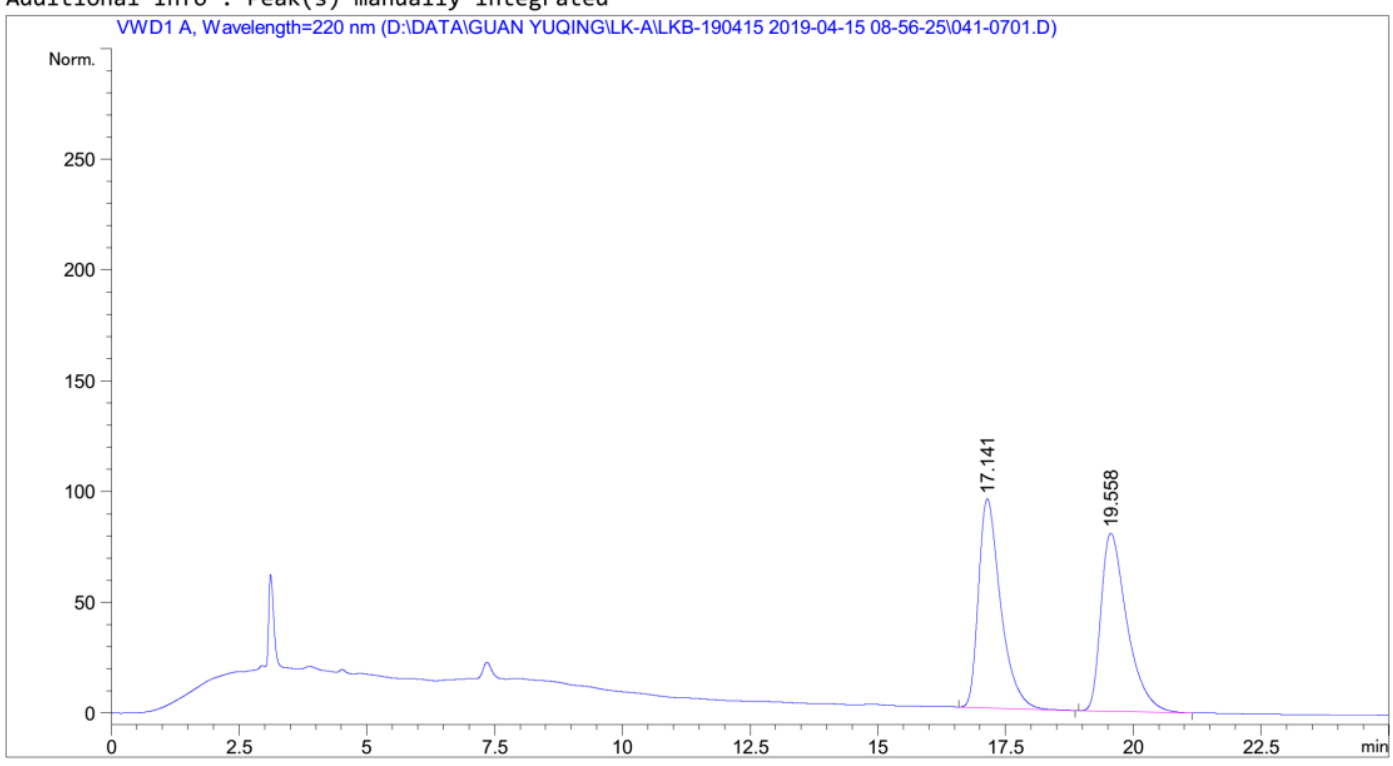

Area Percent Report

$\begin{array}{lll}================================= \\ \text { Sorted By } & : & \text { Signal } \\ \text { Multiplier } & : & 1.0000 \\ \text { Dilution } & : & 1.0000\end{array}$

Use Multiplier \& Dilution Factor with ISTDs

Signal 1: VWD1 A, Wavelength $=220 \mathrm{~nm}$

\begin{tabular}{|c|c|c|c|c|c|}
\hline $\begin{array}{c}\text { Peak } \\
\#\end{array}$ & $\begin{array}{l}\text { RetTime Type } \\
\text { [min] }\end{array}$ & $\begin{array}{l}\text { Width } \\
\text { [min] }\end{array}$ & $\begin{array}{c}\text { Area } \\
{[\mathrm{mAU} * \mathrm{~s}]}\end{array}$ & $\begin{array}{l}\text { Height } \\
\text { [mAU] }\end{array}$ & $\begin{array}{c}\text { Area } \\
\%\end{array}$ \\
\hline-- & $-\ldots-n \mid--$ & - & | & - & | \\
\hline 1 & $17.141 \mathrm{BB}$ & 0.4541 & 2838.71191 & 94.31995 & 49.8352 \\
\hline 2 & $19.558 \mathrm{BB}$ & 0.5361 & 2857.48901 & 80.50407 & 50.1648 \\
\hline Tota & ; : & & 5696.20093 & 174.82403 & \\
\hline
\end{tabular}


Data File D: \DATA\GUAN YUQING\LK-A\LKB-190415 2019-04-15 08-56-25\042-0801.D Sample Name: LSL-4-31-2

\begin{tabular}{|c|c|c|}
\hline Acq. Operator & : & Seq. Line : 8 \\
\hline Acq. Instrument & : Instrument 1 & Location : Vial 42 \\
\hline Injection Date & : 4/15/2019 1:42:02 PM & $\begin{array}{rc}\text { Inj }: & 1 \\
\text { Inj Volume } & : 3.000\end{array}$ \\
\hline
\end{tabular}

Acq. Method : D: \DATA \GUAN YUQING \LK-A \LKB-190415 2019-04-15 08-56-25\VWD-AD(1-2)-95-5-

1ML-3UL-220NM-40MIN.M

Last changed : 6/7/2018 11:25:33 AM

Analysis Method : D: \METHOD \LG VWD-AD(1-2)-80-20-1ML-3UL-210NM-60MIN.M

Last changed : 8/13/2019 5:06:55 PM

(modified after loading)

Additional Info : Peak(s) manually integrated

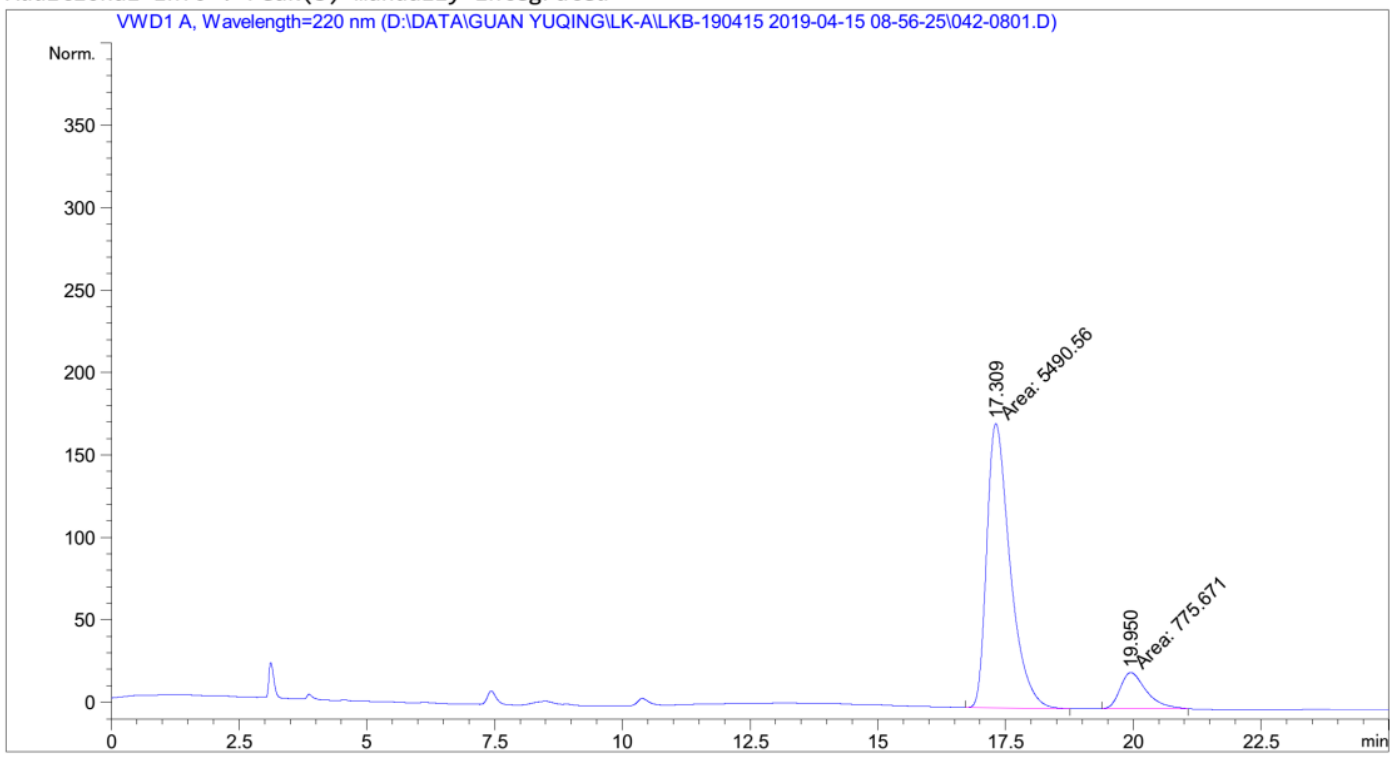

Area Percent Report

$\begin{array}{lll}================================= \\ \text { Sorted By } & : & \text { Signal } \\ \text { Multiplier } & : & 1.0000 \\ \text { Dilution } & : & 1.0000\end{array}$

Use Multiplier \& Dilution Factor with ISTDs

Signal 1: VWD1 A, Wavelength $=220 \mathrm{~nm}$

\begin{tabular}{|c|c|c|c|c|c|c|}
\hline $\begin{array}{c}\text { Peak } \\
\text { \# }\end{array}$ & $\begin{array}{c}\text { RetTime } \\
\text { [min] }\end{array}$ & Type & $\begin{array}{l}\text { Width } \\
\text { [min] }\end{array}$ & $\begin{array}{c}\text { Area } \\
{\left[\mathrm{mAU}^{*} \mathrm{~s}\right]}\end{array}$ & $\begin{array}{l}\text { Height } \\
\text { [mAU] }\end{array}$ & $\begin{array}{c}\text { Area } \\
\%\end{array}$ \\
\hline---1 & $|-\ldots-1|$ & & $-\ldots$ & | & | & | \\
\hline 1 & 17.309 & MM & 0.5305 & 5490.56152 & 172.49892 & 87.6214 \\
\hline 2 & 19.950 & MM & 0.5903 & 775.67078 & 21.89940 & 12.3786 \\
\hline Tota. & & & & 6266.23230 & 194.39832 & \\
\hline
\end{tabular}


<smiles>CC[C@H](Cc1ccccc1)C(=O)N1CCOCC1</smiles>

Data File D: \DATA \LSL\LSL-4-21\LSL-4-31-3 2019-04-13 10-22-57\051-0301.D Sample Name: LSL-4-21-4
Acq. Operator :
Seq. Line : 3
Acq. Instrument : Instrument 2
Location : Vial 51
Injection Date : 4/13/2019 10:47:55 AM
Inj : 1
Inj Volume : $3.000 \mu 1$

Acq. Method : D: \DATA \LSL\LSL-4-21\LSL-4-31-3 2019-04-13 10-22-57\DAD-OD(1-2)-90-10-1ML3UL-ALL-4OMIN.M

Last changed : 11/19/2018 8:48:05 AM

Analysis Method: D: \METHOD \LG\DAD-0J(1-6)-80-20-1ML-5UL-ALL-60MIN.M

Last changed : 4/15/2019 10:10:51 AM

(modified after loading)

Additional Info: Peak(s) manually integrated

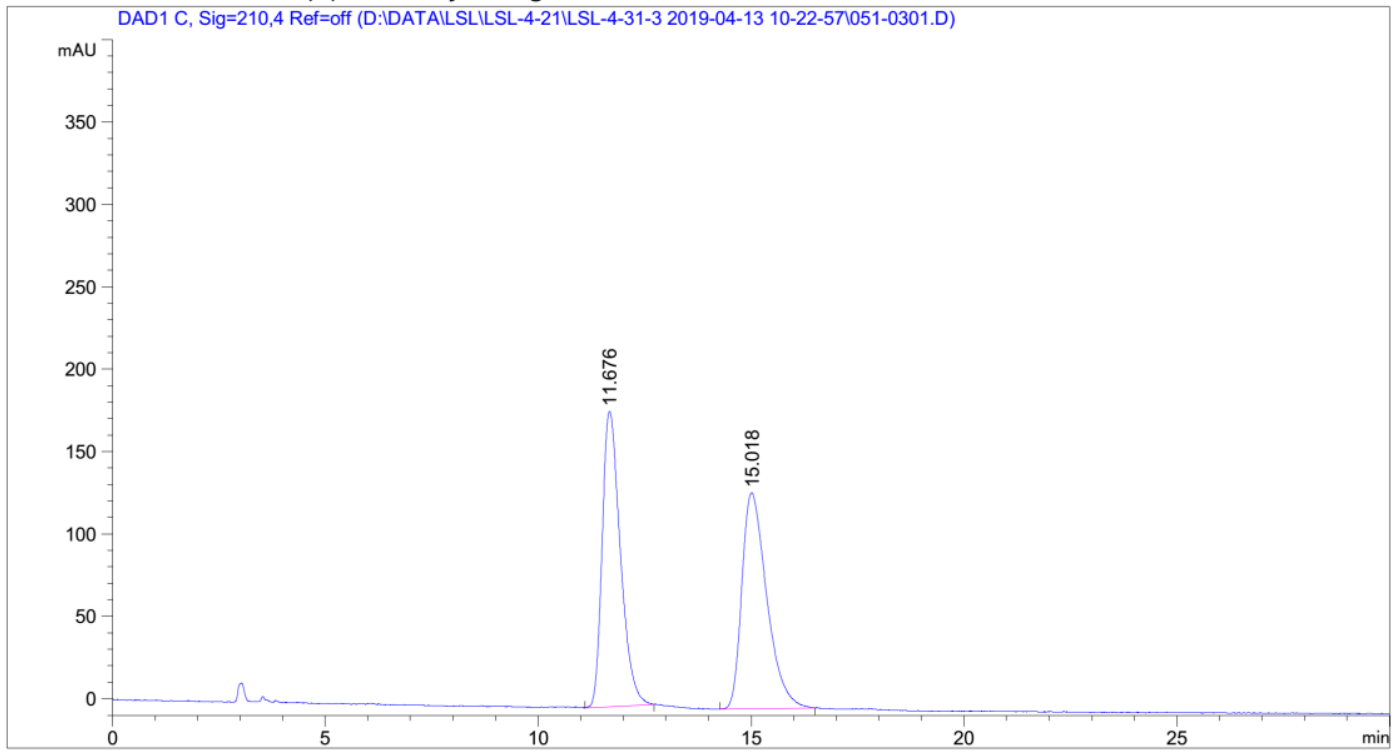

Area Percent Report

$\begin{array}{lll}================================= \\ \text { Sorted By } & : & \text { Signal } \\ \text { Multiplier } & : & 1.0000 \\ \text { Dilution } & : & 1.0000\end{array}$

Use Multiplier \& Dilution Factor with ISTDs

Signal 1: DAD1 C, Sig=210,4 Ref=off

\begin{tabular}{|c|c|c|c|c|c|c|}
\hline $\begin{array}{c}\text { Peak } \\
\#\end{array}$ & $\begin{array}{c}\text { RetTime } \\
\text { [min] }\end{array}$ & Type & $\begin{array}{l}\text { Width } \\
\text { [min] }\end{array}$ & $\begin{array}{c}\text { Area } \\
{\left[\mathrm{mAU}^{*} \mathrm{~s}\right]}\end{array}$ & $\begin{array}{l}\text { Height } \\
\text { [mAU] }\end{array}$ & $\begin{array}{c}\text { Area } \\
\quad \%\end{array}$ \\
\hline--1 & |-.... & & - & $|-\cdots-\ldots|$ & | & - \\
\hline 1 & 11.676 & BV & 0.4404 & 5320.61035 & 179.19835 & 49.9389 \\
\hline 2 & 15.018 & BB & 0.5521 & 5333.63428 & 131.23808 & 50.0611 \\
\hline
\end{tabular}


Data File D: \DATA \LSL\LSL-4-21\LSL-4-31-3 2019-04-13 10-22-57\052-0401.D Sample Name: LSL-4-31-3

\begin{tabular}{|c|c|c|}
\hline Acq. Operator & : & Seq. Line : 4 \\
\hline Acq. Instrument & : Instrument 2 & Location : Vial 52 \\
\hline Injection Date & : 4/13/2019 11:28:52 AM & $\begin{array}{rlc}\text { Inj } & : & 1 \\
\text { Inj Volume } & : & 3.000\end{array}$ \\
\hline
\end{tabular}

Acq. Method : D: \DATA \LSL \LSL-4-21\LSL-4-31-3 2019-04-13 10-22-57\DAD-OD(1-2)-90-10-1ML-

Last changed : 11/19/2018 8:48:05 AM

Analysis Method : D: \METHOD\LG\DAD-0J(1-6)-80-20-1ML-5UL-ALL-60MIN.M

Last changed : 4/15/2019 10:13:15 AM

(modified after loading)

Additional Info : Peak(s) manually integrated

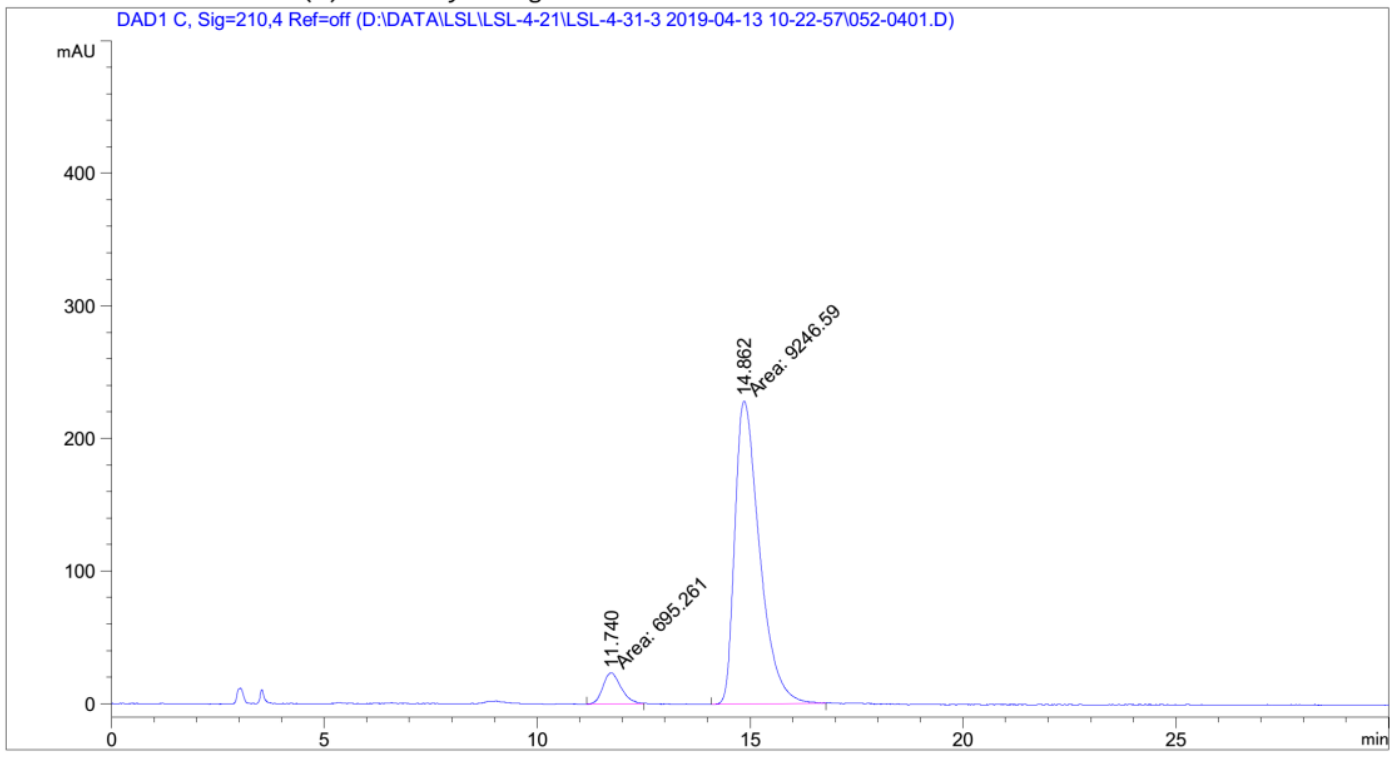

Area Percent Report

$\begin{array}{lll}================================ \\ \text { Sorted By } & : & \text { Signal } \\ \text { Multiplier } & : & 1.0000 \\ \text { Dilution } & : & 1.0000\end{array}$

Use Multiplier \& Dilution Factor with ISTDs

Signal 1: DAD1 C, Sig=210,4 Ref=off

\begin{tabular}{|c|c|c|c|c|c|}
\hline $\begin{array}{c}\text { Peak } \\
\#\end{array}$ & $\begin{array}{l}\text { RetTime Type } \\
\text { [min] }\end{array}$ & $\begin{array}{l}\text { Width } \\
\text { [min] }\end{array}$ & $\begin{array}{c}\text { Area } \\
{\left[\mathrm{mAU}^{*} \mathrm{~s}\right]}\end{array}$ & $\begin{array}{l}\text { Height } \\
\text { [mAU] }\end{array}$ & $\begin{array}{c}\text { Area } \\
\%\end{array}$ \\
\hline 1 & 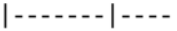 & & - & . & 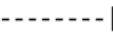 \\
\hline 1 & $11.740 \mathrm{MM}$ & 0.4948 & 695.26068 & 23.41763 & 6.9933 \\
\hline 2 & $14.862 \mathrm{MM}$ & 0.6738 & 9246.58789 & 228.70569 & 93.0067 \\
\hline Total & & & 9941.84857 & 252.12332 & \\
\hline
\end{tabular}

Instrument 2 4/15/2019 10:13:21 AM

Page 1 of 2 
<smiles>CCOC(=O)C(CC=O)Cc1ccc(F)cc1</smiles>

Data File D: \DATA \LSL \LSL-3-196\LSL-3-196 2019-01-20 14-29-19\011-7101.D Sample Name: P-F RAC

\begin{tabular}{|c|c|c|}
\hline Acq. Operator & : & Seq. Line : 71 \\
\hline Acq. Instrument & : Instrument 2 & Location : Vial 11 \\
\hline Injection Date & : 1/22/2019 2:21:00 AM & $\begin{array}{rlc}\text { Inj } & : & 1 \\
\text { Inj Volume } & : & 5.000 \mu \mathrm{l}\end{array}$ \\
\hline
\end{tabular}

Acq. Method : D: \DATA \LSL\LSL-3-196\LSL-3-196 2019-01-20 14-29-19 \DAD-OD(1-2)-98-2-1ML5UL-ALL-40MIN.M

Last changed : 9/21/2018 9:52:07 AM

Analysis Method : D: \METHOD \GUAN YUQING \DAD-0J(1-6)-96-4-0.8ML-5UL-ALL-90MIN.M

Last changed : 1/22/2019 12:22:17 PM

(modified after loading)

Additional Info : Peak(s) manually integrated

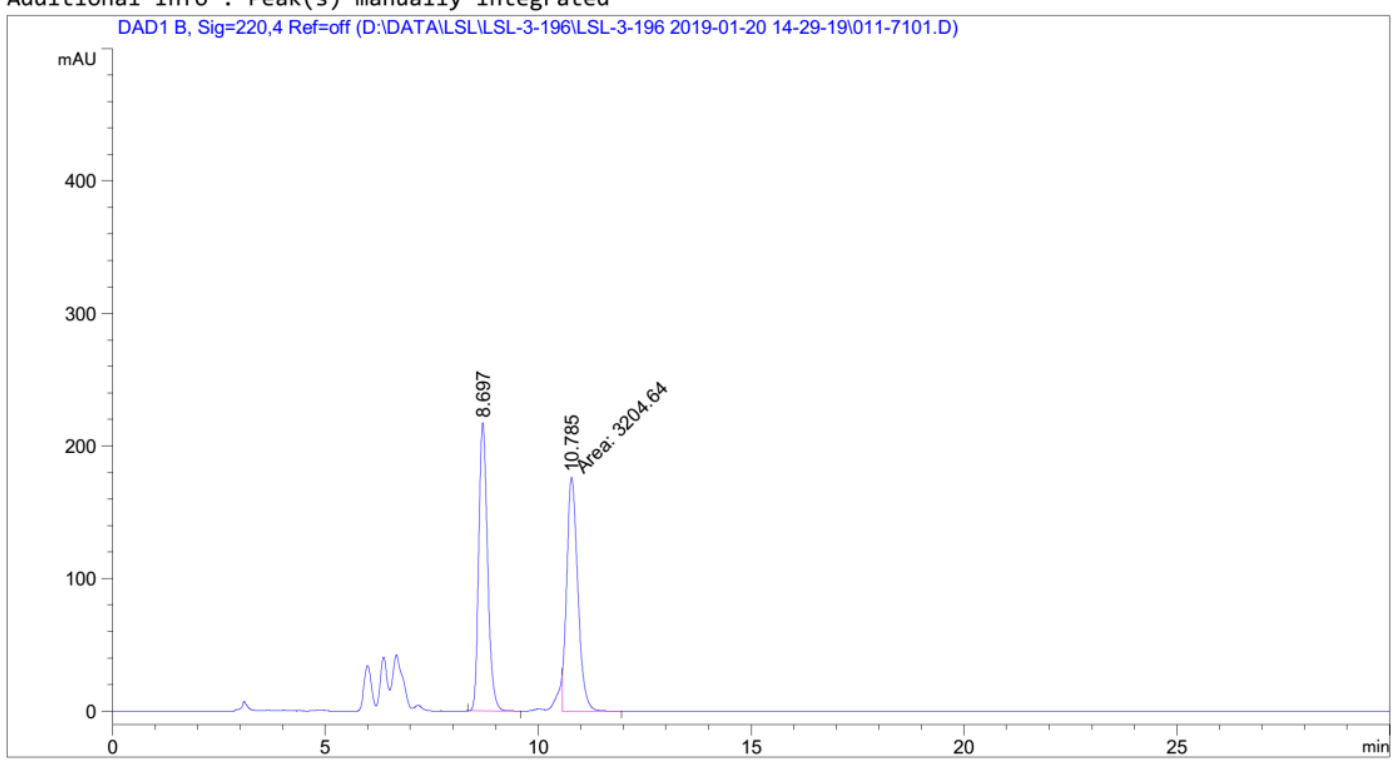

Area Percent Report

$\begin{array}{lll} & & \text { Area Percent } \\ ============================= \\ \text { Sorted By } & : & \text { Signal } \\ \text { Multiplier } & : & 1.0000 \\ \text { Dilution } & : & 1.0000\end{array}$

Use Multiplier \& Dilution Factor with ISTDs

Signal 1: DAD1 B, Sig $=220,4$ Ref $=$ off

\begin{tabular}{|c|c|c|c|c|c|c|}
\hline $\begin{array}{c}\text { Peak } \\
\#\end{array}$ & $\begin{array}{c}\text { RetTime } \\
\text { [min] }\end{array}$ & Type & $\begin{array}{l}\text { Width } \\
\text { [min] }\end{array}$ & $\begin{array}{c}\text { Area } \\
{\left[\mathrm{mAU}^{*} \mathrm{~s}\right]}\end{array}$ & $\begin{array}{l}\text { Height } \\
\text { [mAU] }\end{array}$ & $\begin{array}{c}\text { Area } \\
\%\end{array}$ \\
\hline$\ldots$ & $-\ldots-\ldots$ & & 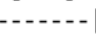 & | & . & | \\
\hline 1 & 8.697 & BB & 0.2201 & 3131.26563 & 217.34355 & 49.4209 \\
\hline 2 & 10.785 & FM & 0.3031 & 3204.64209 & 176.21651 & 50.5791 \\
\hline Tota & 5 : & & & 6335.90771 & 393.56006 & \\
\hline
\end{tabular}


Data File D: \DATA\LSL\LSL-3-196\LSL-3-196 2019-01-20 14-29-19\012-7201.D Sample Name: P-F

\begin{tabular}{|c|c|c|}
\hline $\begin{array}{l}\text { Acq. Operator } \\
\text { Acq. Instrument }\end{array}$ & : Instrument 2 & $\begin{aligned} & \text { Seq. Line : } 72 \\
& \text { Location : Vial } 12\end{aligned}$ \\
\hline Injection Date & $: 1 / 22 / 20193: 02: 01$ AM & $\begin{array}{r:c}\text { Inj }: & 1 \\
\text { Inj Volume } & 5.000\end{array}$ \\
\hline
\end{tabular}

Acq. Method : D: \DATA \LSL\LSL-3-196\LSL-3-196 2019-01-20 14-29-19 \DAD-OD(1-2)-98-2-1ML-

$\begin{aligned} & \text { 5UL-ALL-40MIN.M } \\ \text { Last changed } & \text { 9/21/2018 9:52:07 AM }\end{aligned}$

Analysis Method : D: \METHOD\GUAN YUQING DDAD-0J(1-6)-96-4-0.8ML-5UL-ALL-90MIN.M

Last changed : 1/22/2019 12:24:03 PM

(modified after loading)

Additional Info : Peak(s) manually integrated

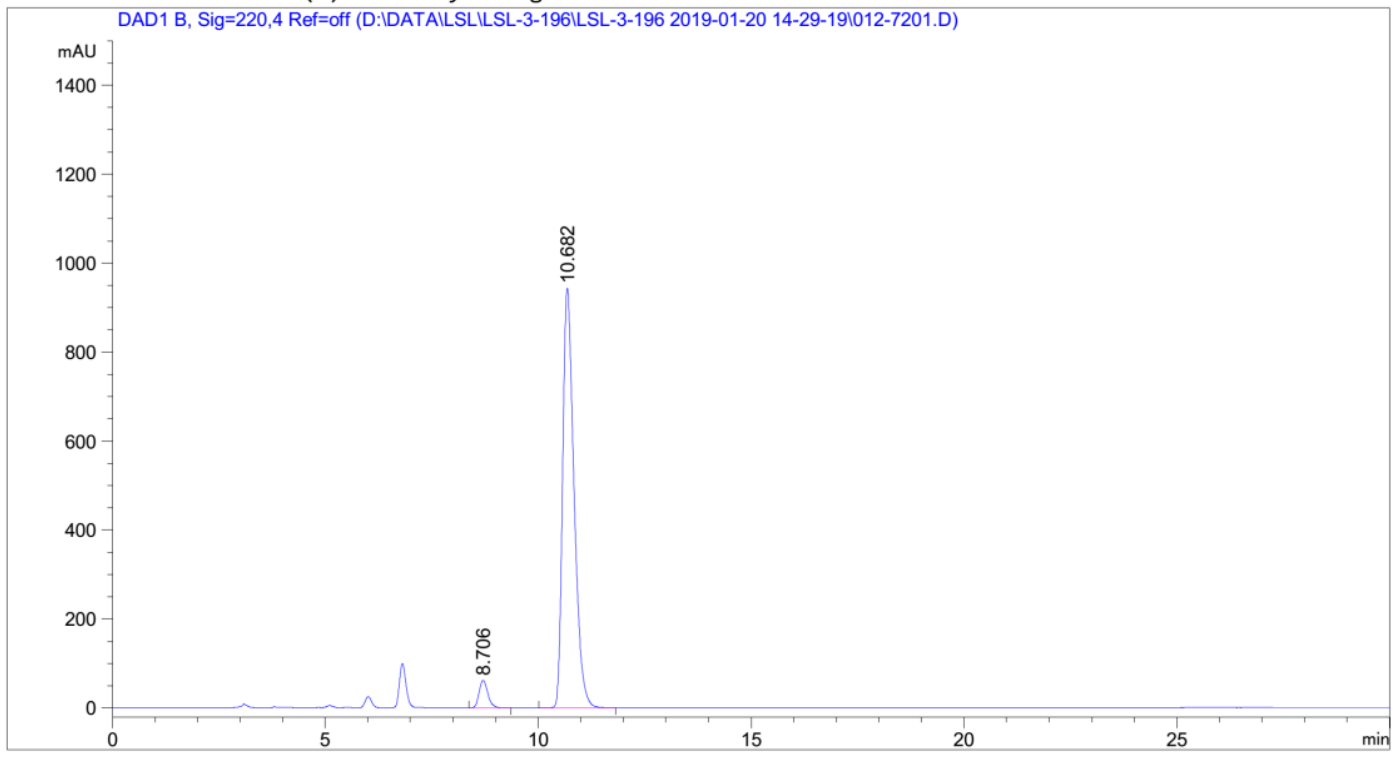

Area Percent Report

$\begin{array}{lll}================================ \\ \text { Sorted By } & : & \text { Signal } \\ \text { Multiplier } & : & 1.0000 \\ \text { Dilution } & : & 1.0000\end{array}$

Use Multiplier \& Dilution Factor with ISTDs

Signal 1: DAD1 B, Sig=220,4 Ref=off

\begin{tabular}{|c|c|c|c|c|c|}
\hline $\begin{array}{c}\text { Peak } \\
\#\end{array}$ & $\begin{array}{l}\text { RetTime Type } \\
\text { [min] }\end{array}$ & $\begin{array}{l}\text { Width } \\
\text { [min] }\end{array}$ & $\begin{array}{c}\text { Area } \\
{\left[\mathrm{mAU}^{*} \mathrm{~s}\right]}\end{array}$ & $\begin{array}{l}\text { Height } \\
{[\mathrm{mAU}]}\end{array}$ & $\begin{array}{c}\text { Area } \\
\%\end{array}$ \\
\hline 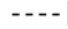 & $\mid \cdots-\cdots$ & & - & 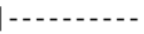 & 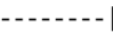 \\
\hline 1 & $8.706 \mathrm{BB}$ & 0.2199 & 883.87872 & 62.14118 & 4.8001 \\
\hline 2 & $10.682 \mathrm{BB}$ & 0.2848 & $1.75298 \mathrm{e} 4$ & 943.52692 & 95.1999 \\
\hline Tota. & & & $1.84136 \mathrm{e} 4$ & 1005.66809 & \\
\hline
\end{tabular}


<smiles>CCOC(=O)C(CC=O)Cc1ccc(Cl)cc1</smiles>

Data File D: \DATA\LSL\LSL-3-196\LSL-3-196 2019-01-20 14-29-19\013-7301.D Sample Name: P-CI-RAC

\begin{tabular}{|c|c|c|}
\hline Acq. Operator & : & Seq. Line : 73 \\
\hline Acq. Instrument & : Instrument 2 & Location : Vial 13 \\
\hline Injection Date & $: 1 / 22 / 20193: 43: 00$ AM & $\begin{array}{rcc}\text { Inj } & : & 1 \\
\text { Inj Volume } & : & 5.000 \mu l\end{array}$ \\
\hline
\end{tabular}

Acq. Method : D: \DATA \LSL\LSL-3-196\LSL-3-196 2019-01-20 14-29-19 \DAD-OD(1-2)-98-2-1ML5UL-ALL-40MIN.M

Last changed : 9/21/2018 9:52:07 AM

Analysis Method : D: \METHOD \GUAN YUQING \DAD-0J(1-6)-96-4-0.8ML-5UL-ALL-90MIN.M

Last changed : 1/22/2019 12:27:13 PM

(modified after loading)

Additional Info : Peak(s) manually integrated

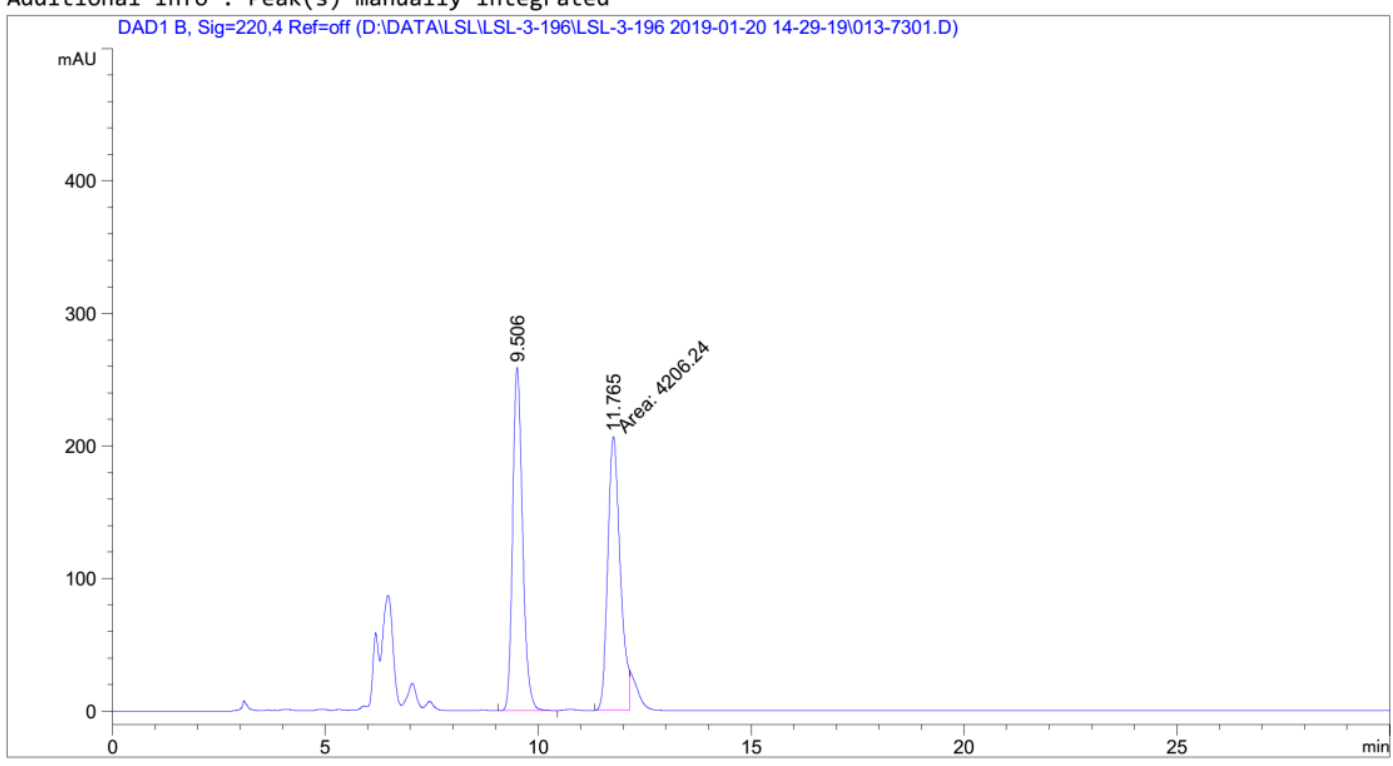

Area Percent Report

$\begin{array}{lll} & & \text { Area Percen } \\ ============================== \\ \text { Sorted By } & : & \text { Signal } \\ \text { Multiplier } & : & 1.0000 \\ \text { Dilution } & : & 1.0000\end{array}$

Use Multiplier \& Dilution Factor with ISTDs

Signal 1: DAD1 B, Sig $=220,4$ Ref $=$ off

\begin{tabular}{|c|c|c|c|c|c|c|}
\hline $\begin{array}{c}\text { Peak } \\
\#\end{array}$ & $\begin{array}{c}\text { RetTime } \\
\text { [min] }\end{array}$ & Type & $\begin{array}{l}\text { Width } \\
\text { [min] }\end{array}$ & $\begin{array}{c}\text { Area } \\
{[\mathrm{mAU} * \mathrm{~s}]}\end{array}$ & $\begin{array}{l}\text { Height } \\
{[\mathrm{mAU}]}\end{array}$ & $\begin{array}{c}\text { Area } \\
\%\end{array}$ \\
\hline$\ldots$ & $\ldots$ & & (n........ & | & | & | \\
\hline 1 & 9.506 & BB & 0.2464 & 4181.17725 & 258.89526 & 49.8506 \\
\hline 2 & 11.765 & MF & 0.3397 & 4206.24316 & 206.34270 & 50.1494 \\
\hline Tota & s : & & & 8387.42041 & 465.23796 & \\
\hline
\end{tabular}


Data File D: \DATA \LSL\LSL-3-196\LSL-3-196 2019-01-20 14-29-19\014-7401.D Sample Name: P-Cl

\begin{tabular}{|c|c|c|}
\hline $\begin{array}{l}\text { Acq. Operator } \\
\text { Acq. Instrument }\end{array}$ & $\begin{array}{l}: \\
: \text { Instrument } 2\end{array}$ & $\begin{aligned} & \text { Seq. Line }: 74 \\
& \text { Location : Vial } 14\end{aligned}$ \\
\hline Injection Date & : 1/22/2019 4:23:59 AM & 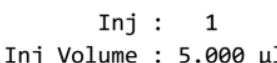 \\
\hline
\end{tabular}

Acq. Method : D: \DATA \LSL\LSL-3-196\LSL-3-196 2019-01-20 14-29-19 \DAD-OD(1-2)-98-2-1ML-

$\begin{aligned} & \text { 5UL-ALL-40MIN.M } \\ \text { Last changed } & \text { : } 9 / 21 / 2018 \text { 9:52:07 AM }\end{aligned}$

Analysis Method : D: \METHOD\GUAN YUQING DDAD-0J(1-6)-96-4-0.8ML-5UL-ALL-90MIN.M

Last changed : 1/22/2019 12:28:48 PM

(modified after loading)

Additional Info : Peak(s) manually integrated

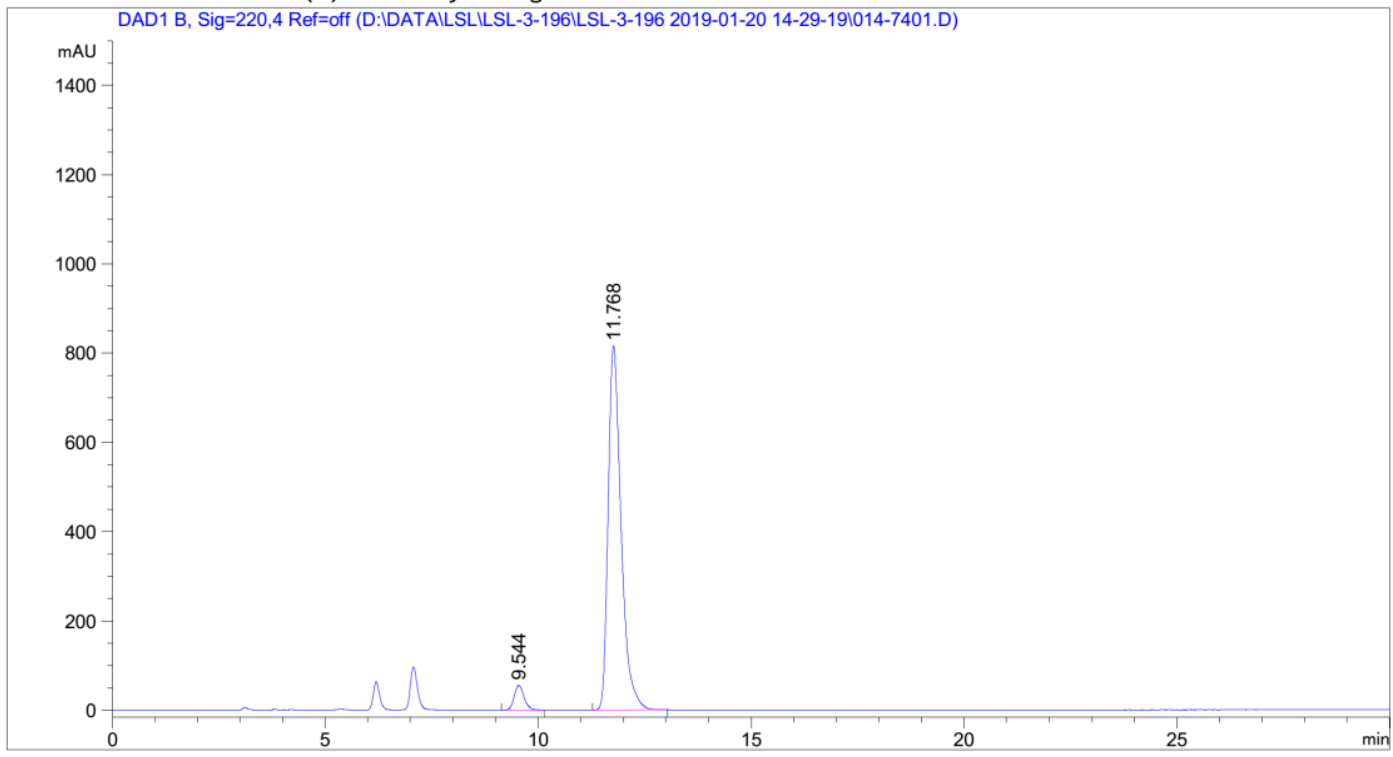

Area Percent Report

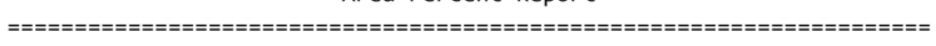

$\begin{array}{lll}\text { Sorted By } & : & \text { Signal } \\ \text { Multiplier } & : & 1.0000 \\ \text { Dilution } & : & 1.0000\end{array}$

Use Multiplier \& Dilution Factor with ISTDs

Signal 1: DAD1 B, Sig=220,4 Ref=off

\begin{tabular}{cccccc}
$\begin{array}{c}\text { Peak RetTime Type } \\
\text { W }\end{array}\left[\begin{array}{c}\text { Width } \\
{[\mathrm{min}]}\end{array}\right.$ & $\begin{array}{c}\text { Area } \\
{[\mathrm{mAU} \text { s }]}\end{array}$ & $\begin{array}{c}\text { Height } \\
{[\mathrm{mAU}]}\end{array}$ & \multicolumn{1}{c}{$\begin{array}{c}\text { Area } \\
\%\end{array}$} \\
\hline 1 & 9.544 BB & 0.2478 & 889.68787 & 55.27421 & 4.9121 \\
2 & 11.768 BB & 0.3202 & $1.72224 \mathrm{e} 4$ & 815.96490 & 95.0879 \\
& & & & \\
Totals : & & $1.81121 \mathrm{e} 4$ & 871.23912 &
\end{tabular}


<smiles>CCCOC(=O)[C@H](CC=O)Cc1ccc(Br)cc1</smiles>

Data File D: \DATA \LSL\LSL-3-196\LSL-3-196 2019-01-20 14-29-19\051-0301.D Sample Name: LSL-3-168-3
Acq. Operator :
Acq. Instrument : Instrument 2
Seq. Line : 3
Location : Vial 51
Injection Date : 1/20/2019 2:52:32 PM
Inj : 1
Inj Volume : $5.000 \mu \mathrm{l}$

Acq. Method : D: \DATA \LSL\LSL-3-196\LSL-3-196 2019-01-20 14-29-19 \DAD-OD(1-2)-98-2-1ML5UL-ALL-40MIN.M

Last changed : 9/21/2018 9:52:07 AM

Analysis Method : D: \METHOD \LG\DAD-OD(1-2)-99-1-0.5ML-5UL-ALL-100MIN.M

Last changed

1/21/2019 9:26:19 PM

(modified after loading)

Additional Info : Peak(s) manually integrated

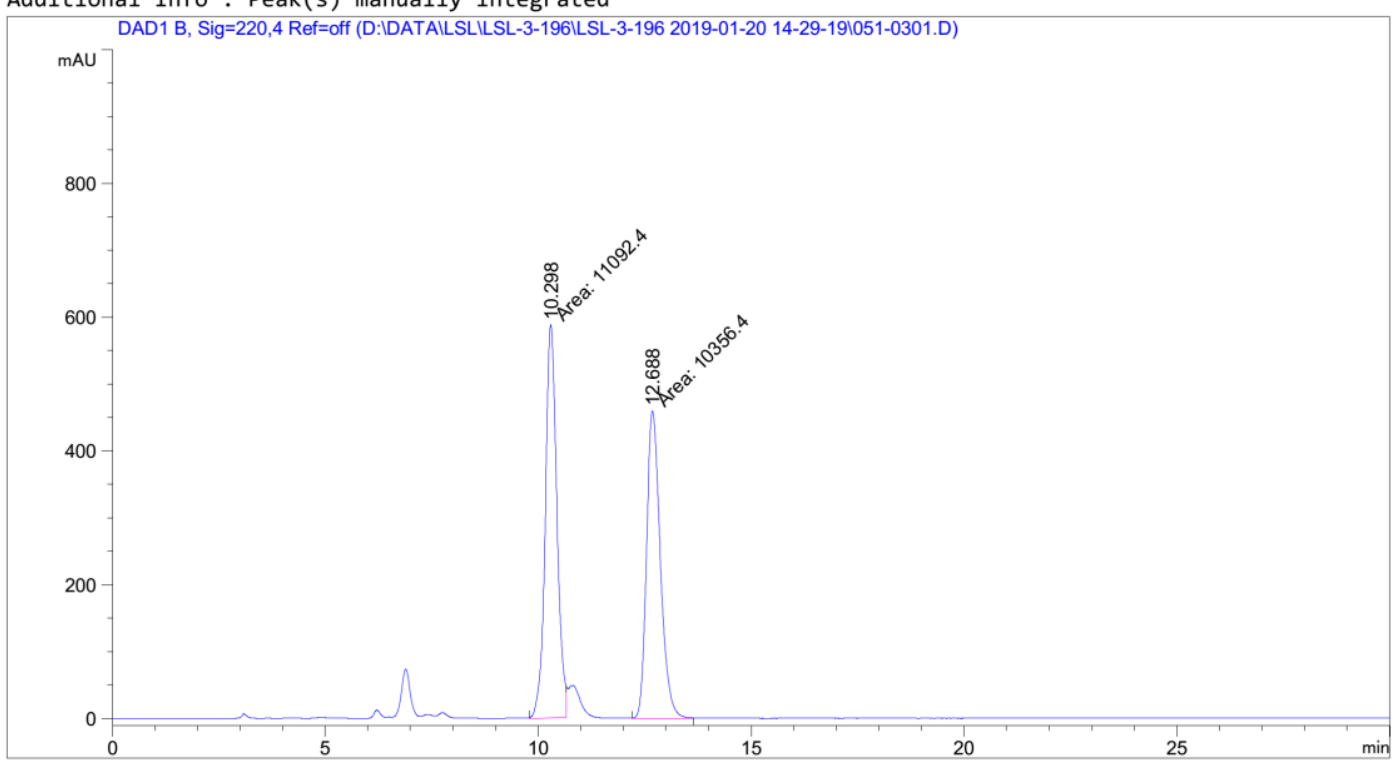

Area Percent Report

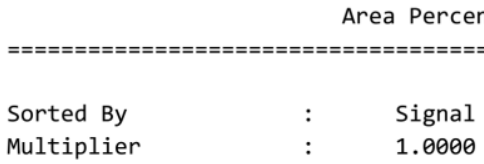

Multiplier

Dilution : 1.0000

Use Multiplier \& Dilution Factor with ISTDs

Signal 1: DAD1 B, Sig=220,4 Ref=off

\begin{tabular}{|c|c|c|c|c|c|c|}
\hline $\begin{array}{c}\text { Peak } \\
\#\end{array}$ & $\begin{array}{c}\text { RetTime } \\
\text { [min] }\end{array}$ & Type & $\begin{array}{l}\text { Width } \\
\text { [min] }\end{array}$ & $\begin{array}{c}\text { Area } \\
{[\mathrm{mAU} * \mathrm{~s}]}\end{array}$ & $\begin{array}{l}\text { Height } \\
{[\mathrm{mAU}]}\end{array}$ & $\begin{array}{c}\text { Area } \\
\%\end{array}$ \\
\hline$-\ldots$ & $\ldots$ & & | & | & $-\ldots$ & | \\
\hline 1 & 10.298 & MF & 0.3145 & $1.10924 \mathrm{e} 4$ & 587.83020 & 51.7157 \\
\hline 2 & 12.688 & MM & 0.3757 & $1.03564 \mathrm{e} 4$ & 459.40488 & 48.2843 \\
\hline Tota & : & & & $2.14488 \mathrm{e} 4$ & 1047.23508 & \\
\hline
\end{tabular}


Data File D: \DATA \LSL\LSL-3-196\LSL-3-196 2019-01-20 14-29-19\052-0401.D Sample Name: LSL-3-196-3

\begin{tabular}{|c|c|c|}
\hline Acq. Operator & : & Seq. Line : 4 \\
\hline Acq. Instrument & : Instrument 2 & Location : Vial 52 \\
\hline Injection Date & : 1/20/2019 3:33:33 PM & 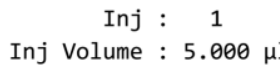 \\
\hline
\end{tabular}

Acq. Method : D: \DATA \LSL\LSL-3-196\LSL-3-196 2019-01-20 14-29-19\DAD-OD(1-2)-98-2-1ML-

$\begin{aligned} & 5 U L-A L L-40 M I N . M \\ & \text { Last changed } \quad: \quad 9 / 21 / 2018 \text { 9:52:07 AM }\end{aligned}$

Analysis Method : D: \METHOD\LG\DAD-OD(1-2)-99-1-0.5ML-5UL-ALL-100MIN.M

Last changed : 1/21/2019 9:54:04 PM

(modified after loading)

Additional Info : Peak(s) manually integrated

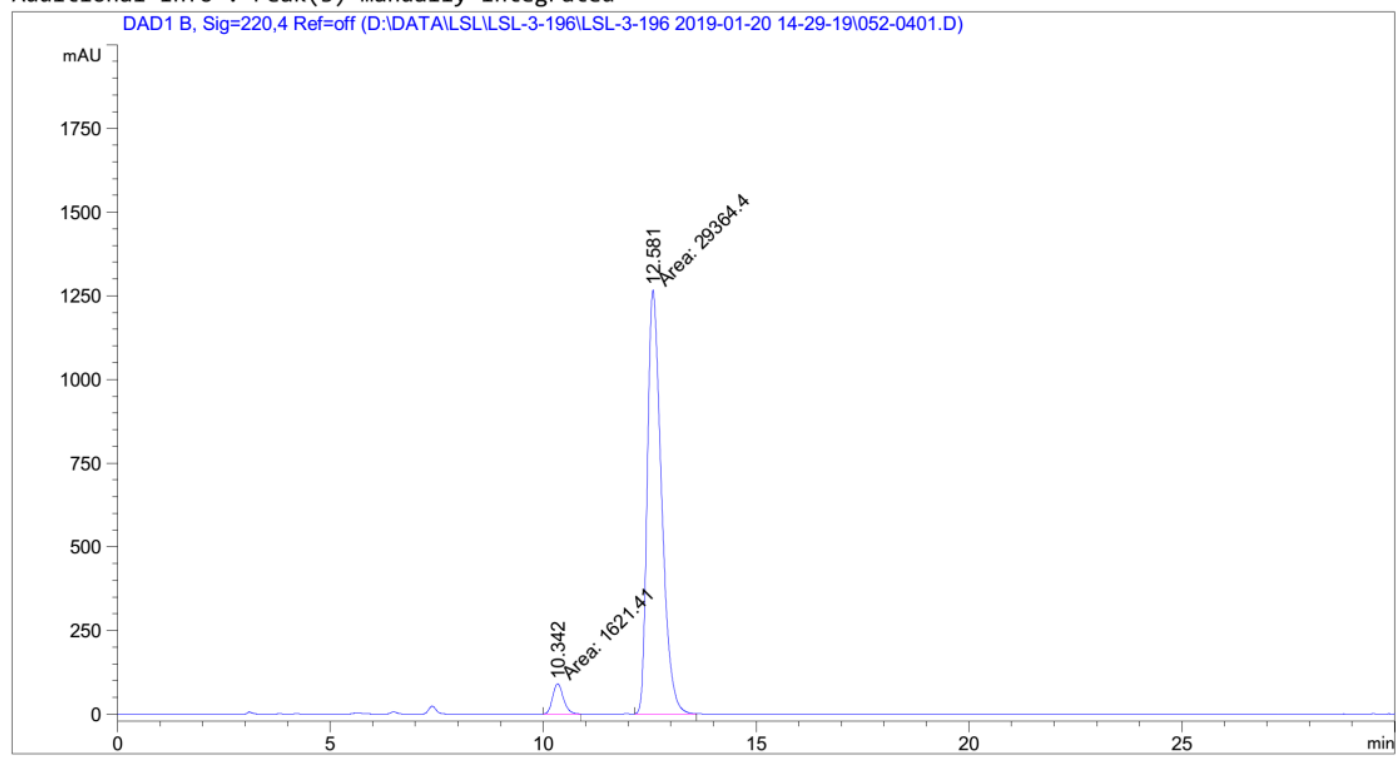

Area Percent Report

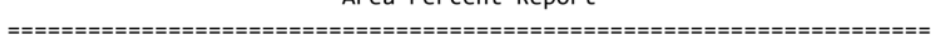

$\begin{array}{lll}\text { Sorted By } & : & \text { Signal } \\ \text { Multiplier } & : & 1.0000 \\ \text { Dilution } & : & 1.0000\end{array}$

Use Multiplier \& Dilution Factor with ISTDs

Signal 1: DAD1 B, Sig=220,4 Ref=off

\begin{tabular}{|c|c|c|c|c|c|}
\hline $\begin{array}{c}\text { Peak } \\
\text { \# }\end{array}$ & $\begin{array}{l}\text { RetTime Type } \\
\text { [min] }\end{array}$ & $\begin{array}{l}\text { Width } \\
\text { [min] }\end{array}$ & $\begin{array}{c}\text { Area } \\
{[\mathrm{mAU} * \mathrm{~s}]}\end{array}$ & $\begin{array}{l}\text { Height } \\
\text { [mAU] }\end{array}$ & $\begin{array}{c}\text { Area } \\
\%\end{array}$ \\
\hline---1 & $|-\ldots-n|--$ & $\ldots$ & | & | & $-\ldots$ \\
\hline 1 & $10.342 \mathrm{MM}$ & 0.2976 & 1621.41370 & 90.80077 & 5.2328 \\
\hline 2 & $12.581 \mathrm{MM}$ & 0.3862 & $2.93644 \mathrm{e} 4$ & 1267.12195 & 94.7672 \\
\hline Total & 1s: & & $3.09858 \mathrm{e} 4$ & 1357.92272 & \\
\hline
\end{tabular}


<smiles>CCCCCCC(=O)OCCCCC(=O)OC</smiles>

Data File D: \DATA \LSLILSL-4-42\LSL-4-42-4 2019-04-26 22-23-46\021-0301.D Sample Name: LSL-4-39-5

\begin{tabular}{|c|c|c|}
\hline Acq. Operator & : & Seq. Line : 3 \\
\hline Acq. Instrument & : Instrument 2 & Location : Vial 21 \\
\hline Injection Date & : 4/26/2019 10:47:41 PM & $\begin{array}{rc}\text { Inj }: & 1 \\
\text { Inj Volume } & : 5.000 \mu\end{array}$ \\
\hline
\end{tabular}

Acq. Method : D: \DATA \LSL \LSL-4-42\LSL-4-42-4 2019-04-26 22-23-46\DAD-OD(1-2)-95-5-1ML5UL-ALL-60MIN.M

Last changed : 6/21/2018 2:29:29 PM

Analysis Method: D: \METHOD \HZY \VWD-AS(1-6)-85-15-1ML-1UL-220NM-25MIN.M

Last changed : 4/27/2019 10:33:13 PM

(modified after loading)

Additional Info : Peak(s) manually integrated

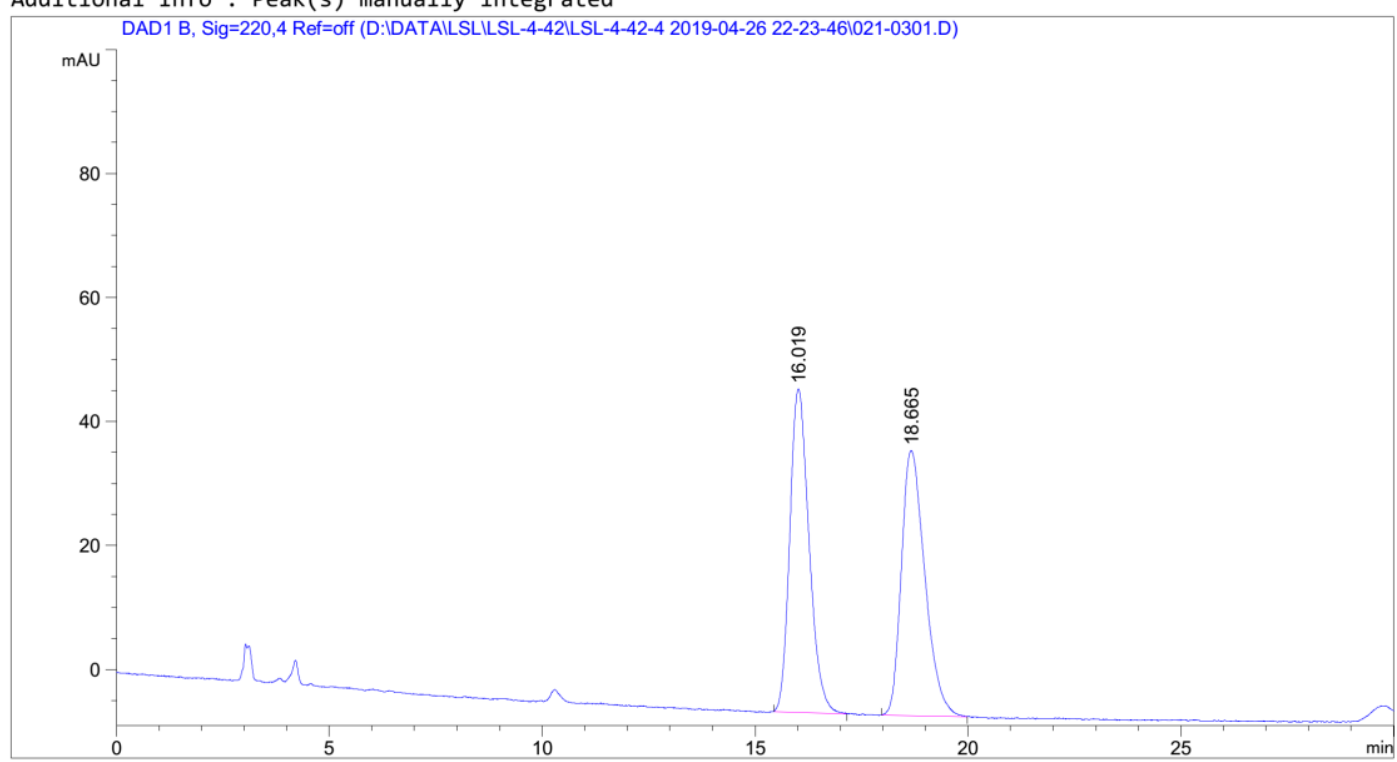

Area Percent Report

$\begin{array}{lll}================================= \\ \text { Sorted By } & : & \text { Signal } \\ \text { Multiplier } & : & 1.0000 \\ \text { Dilution } & : & 1.0000\end{array}$

Use Multiplier \& Dilution Factor with ISTDs

Signal 1: DAD1 B, Sig $=220,4$ Ref $=$ off

\begin{tabular}{|c|c|c|c|c|c|}
\hline $\begin{array}{c}\text { Peak } \\
\#\end{array}$ & $\begin{array}{l}\text { RetTime Type } \\
\text { [min] }\end{array}$ & $\begin{array}{l}\text { Width } \\
\text { [min] }\end{array}$ & $\begin{array}{c}\text { Area } \\
{[\mathrm{mAU} * \mathrm{~s}]}\end{array}$ & $\begin{array}{l}\text { Height } \\
\text { [mAU] }\end{array}$ & $\begin{array}{c}\text { Area } \\
\%\end{array}$ \\
\hline 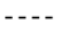 & $\mid--1-1$ & $-\cdots-1$ & | & | & | \\
\hline 1 & 16.019 ВВ & 0.4441 & 1636.83679 & 52.16442 & 49.9188 \\
\hline 2 & 18.665 BВ & 0.5100 & 1642.15942 & 42.76224 & 50.0812 \\
\hline Tota & Is: & & 3278.99622 & 94.92666 & \\
\hline
\end{tabular}


Data File D: \DATA \LSL\LSL-4-42\LSL-4-42-4 2019-04-26 22-23-46\023-0401.D Sample Name: LSL-4-42-4

\begin{tabular}{|c|c|c|}
\hline Acq. Operator & : & Seq. Line : 4 \\
\hline Acq. Instrument & : Instrument 2 & Location : Vial 23 \\
\hline Injection Date & : 4/26/2019 11:48:42 PM & $\begin{array}{rc}\text { Inj }: & 1 \\
\text { Inj Volume } & : 5.000 \mu\end{array}$ \\
\hline
\end{tabular}

Acq. Method : D: \DATA \LSL \LSL-4-42\LSL-4-42-4 2019-04-26 22-23-46\DAD-OD(1-2)-95-5-1ML-

$\begin{aligned} & 5 U L-A L L-60 M I N . M \\ & \text { Last changed } \quad: \quad 6 / 21 / 2018 \quad 2: 29: 29 \text { PM }\end{aligned}$

Analysis Method : D: \METHOD\HZY \VWD-AS(1-6)-85-15-1ML-1UL-220NM-25MIN.M

Last changed : 4/27/2019 10:34:45 PM

(modified after loading)

Additional Info : Peak(s) manually integrated

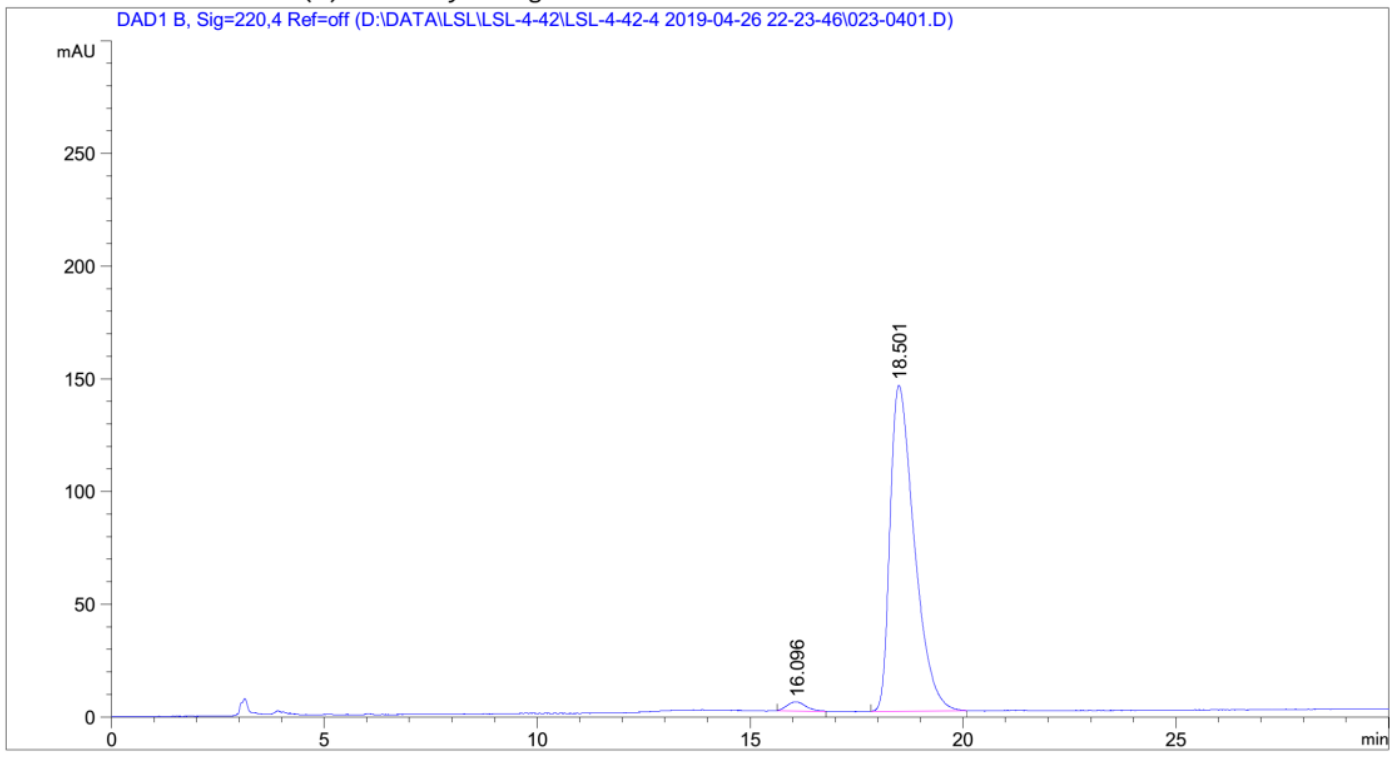

Area Percent Report

$\begin{array}{lll}================================ \\ \text { Sorted By } & : & \text { Signal } \\ \text { Multiplier } & : & 1.0000 \\ \text { Dilution } & : & 1.0000\end{array}$

Use Multiplier \& Dilution Factor with ISTDs

Signal 1: DAD1 B, Sig=220,4 Ref=off

\begin{tabular}{|c|c|c|c|c|c|}
\hline $\begin{array}{c}\text { Peak } \\
\#\end{array}$ & $\begin{array}{l}\text { RetTime Type } \\
\text { [min] }\end{array}$ & $\begin{array}{l}\text { Width } \\
\text { [min] }\end{array}$ & $\begin{array}{c}\text { Area } \\
{[\mathrm{mAU} * \mathrm{~s}]}\end{array}$ & $\begin{array}{l}\text { Height } \\
\text { [mAU] }\end{array}$ & $\begin{array}{c}\text { Area } \\
\%\end{array}$ \\
\hline$-\ldots$ & 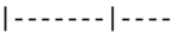 & & - & $--\ldots-n \mid$ & $-\ldots$ \\
\hline 1 & 16.096 ВВ & 0.3628 & 120.89717 & 4.02056 & 2.0681 \\
\hline 2 & $18.501 \mathrm{BB}$ & 0.5697 & 5725.02637 & 144.65211 & 97.9319 \\
\hline Tota] & ls : & & 5845.92354 & 148.67267 & \\
\hline
\end{tabular}


<smiles>CCCOC(=O)C(CC=O)Cc1cccc(OC)c1</smiles>

Data File D: \DATA \LSL \LSL-3-196\LSL-3-196 2019-01-20 14-29-19\055-5601.D Sample Name: LSL-3-176-2-2
Acq. Operator
Acq. Instrument : Instrument 2
Injection Date : 1/21/2019 4:45:24 PM
Seq. Line : 56
Location : Vial 55
Inj : 1
Inj Volume : $5.000 \mu \mathrm{l}$

Acq. Method : D: \DATA \LSL\LSL-3-196\LSL-3-196 2019-01-20 14-29-19 \DAD-OD(1-2)-99-1-1ML5UL-ALL-60MIN.M

Last changed : 5/29/2018 11:41:42 AM

Analysis Method: D: \METHOD $\backslash$ LG \DAD-OD(1-2)-99-1-0.5ML-5UL-ALL-100MIN.M

Last changed

: 1/21/2019 9:15:49 PM

(modified after loading)

Additional Info : Peak(s) manually integrated

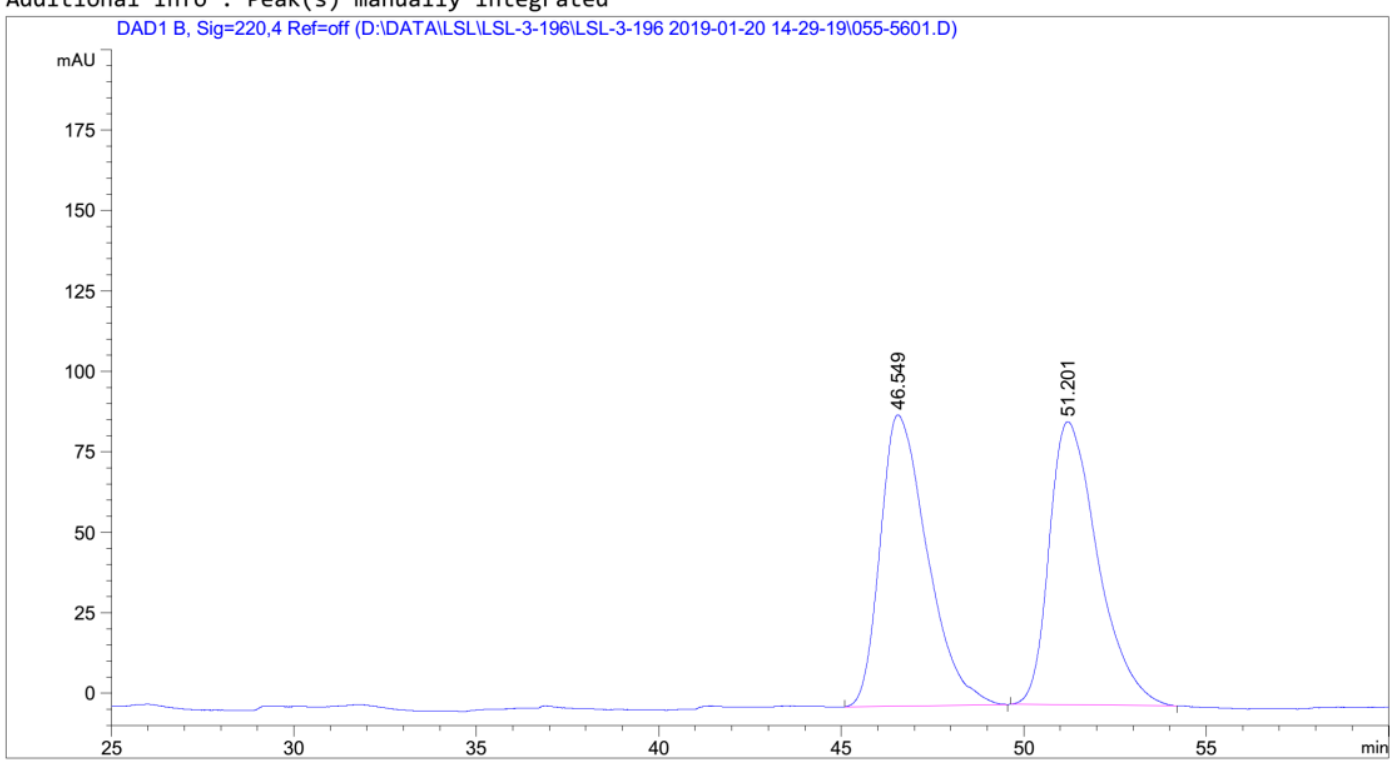

Area Percent Report

$\begin{array}{lll}================================= \\ \text { Sorted By } & : & \text { Signal } \\ \text { Multiplier } & : & 1.0000 \\ \text { Dilution } & : & 1.0000\end{array}$

Use Multiplier \& Dilution Factor with ISTDs

Signal 1: DAD1 B, Sig=220,4 Ref=off

\begin{tabular}{|c|c|c|c|c|c|c|}
\hline $\begin{array}{c}\text { Peak } \\
\text { \# }\end{array}$ & $\begin{array}{c}\text { RetTime } \\
\text { [min] }\end{array}$ & Type & $\begin{array}{l}\text { Width } \\
\text { [min] }\end{array}$ & $\begin{array}{c}\text { Area } \\
\text { [mAU*s] }\end{array}$ & $\begin{array}{l}\text { Height } \\
\text { [mAU] }\end{array}$ & $\begin{array}{c}\text { Area } \\
\%\end{array}$ \\
\hline & & & & $-\cdots$ & & -- \\
\hline 1 & 46.549 & BB & 1.1849 & 8106.61768 & 90.51544 & 49.8825 \\
\hline 2 & 51.201 & BB & 1.2340 & 8144.81543 & 87.98081 & 50.1175 \\
\hline
\end{tabular}

Totals : $\quad 1.62514 \mathrm{e} 4 \quad 178.49625$ 
Data File D: \DATA \LSL\LSL-3-196\LSL-3-196 2019-01-20 14-29-19\056-5701.D Sample Name: LSL-3-196-5-2

\begin{tabular}{|c|c|c|}
\hline Acq. Operator & : & Seq. Line : 57 \\
\hline Acq. Instrument & : Instrument 2 & Location : Vial 56 \\
\hline Injection Date & : 1/21/2019 5:46:25 PM & $\begin{array}{rlc}\text { Inj } & : & 1 \\
\text { Inj Volume } & : & 5.000 \mu\end{array}$ \\
\hline
\end{tabular}

Acq. Method : D: \DATA \LSL\LSL-3-196\LSL-3-196 2019-01-20 14-29-19 \DAD-OD(1-2)-99-1-1ML-

$\begin{aligned} & \text { 5UL-ALL-60MIN.M } \\ \text { Last changed } & \text { 5/29/2018 11:41:42 AM }\end{aligned}$

Analysis Method : D: \METHOD\LG\DAD-OD(1-2)-99-1-0.5ML-5UL-ALL-100MIN.M

Last changed : 1/21/2019 9:22:51 PM

(modified after loading)

Additional Info : Peak(s) manually integrated

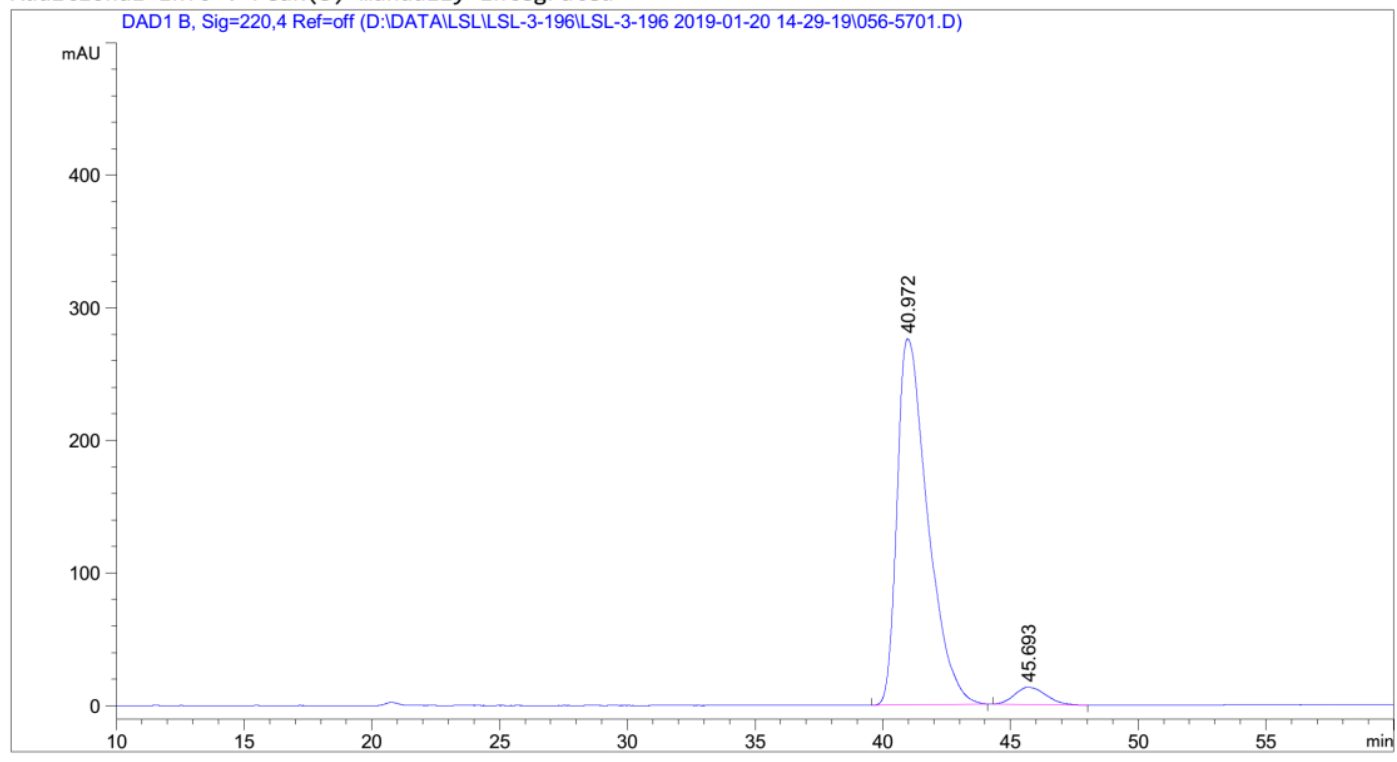

Area Percent Report

$\begin{array}{lll}================================ \\ \text { Sorted By } & : & \text { Signal } \\ \text { Multiplier } & : & 1.0000 \\ \text { Dilution } & : & 1.0000\end{array}$

Use Multiplier \& Dilution Factor with ISTDs

Signal 1: DAD1 B, Sig=220,4 Ref=off

\begin{tabular}{|c|c|c|c|c|c|c|}
\hline $\begin{array}{c}\text { Peak } \\
\#\end{array}$ & $\begin{array}{c}\text { RetTime } \\
\text { [min] }\end{array}$ & Type & $\begin{array}{l}\text { Width } \\
\text { [min] }\end{array}$ & $\begin{array}{c}\text { Area } \\
\text { [mAU*s] }\end{array}$ & $\begin{array}{l}\text { Height } \\
{[\mathrm{mAU}]}\end{array}$ & $\begin{array}{c}\text { Area } \\
\%\end{array}$ \\
\hline$\ldots$ & & & - & | & - & - \\
\hline 1 & 40.972 & BB & 1.1852 & $2.29957 \mathrm{e} 4$ & 276.23962 & 95.3650 \\
\hline 2 & 45.693 & BB & 0.9984 & 1117.64856 & 13.13866 & 4.6350 \\
\hline Tota] & : & & & $2.41133 \mathrm{e} 4$ & 289.37828 & \\
\hline
\end{tabular}


<smiles>CCCOC(=O)C(CC=O)Cc1ccc(OC)cc1</smiles>

20

Data File D: \DATA \LSL\LSL-3-196\LSL-3-196 2019-01-20 14-29-19\057-0901.D Sample Name: LSL-3-176-3

\begin{tabular}{|c|c|c|}
\hline Acq. Operator & $:$ & Seq. Line : 9 \\
\hline Acq. Instrument & : Instrument 2 & Location : Vial 57 \\
\hline Injection Date & : 1/20/2019 6:58:39 PM & 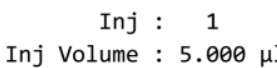 \\
\hline
\end{tabular}

Acq. Method : D: \DATA \LSL\LSL-3-196\LSL-3-196 2019-01-20 14-29-19 \DAD-OD(1-2)-98-2-1ML5UL-ALL-40MIN.M

Last changed : 9/21/2018 9:52:07 AM

Analysis Method : D: \METHOD \LG\DAD-OD(1-2)-99-1-0.5ML-5UL-ALL-100MIN.M

Last changed : 1/21/2019 10:00:12 PM

(modified after loading)

Additional Info: $\operatorname{Peak}(\mathrm{s})$ manually integrated

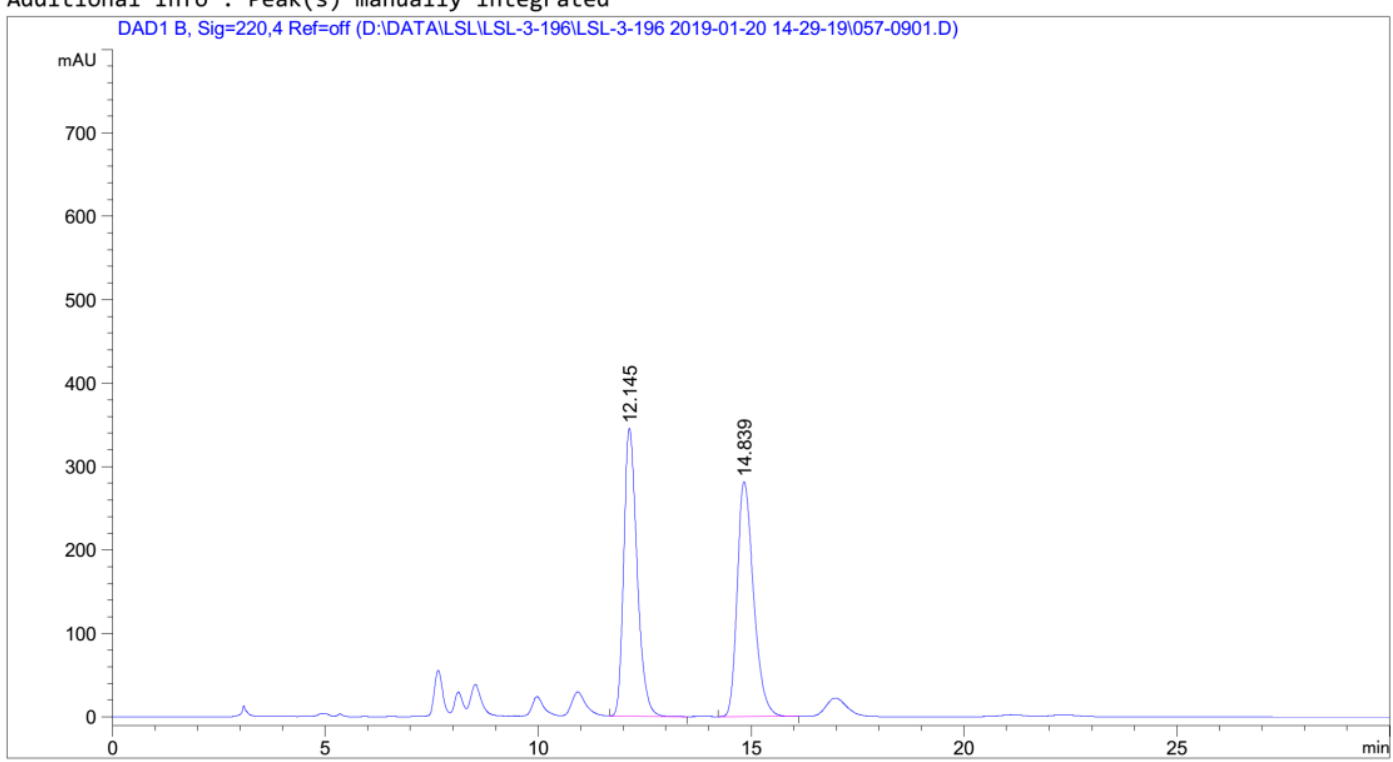

Area Percent Report

\begin{tabular}{|c|c|c|}
\hline Sorted By & : & Signal \\
\hline Multiplier & : & 1.0000 \\
\hline Dilution & : & 1.0000 \\
\hline
\end{tabular}

Use Multiplier \& Dilution Factor with ISTDs

Signal 1: DAD1 B, Sig=220,4 Ref=off

\begin{tabular}{|c|c|c|c|c|c|c|}
\hline $\begin{array}{c}\text { Peak } \\
\#\end{array}$ & $\begin{array}{c}\text { RetTime } \\
\text { [min] }\end{array}$ & Type & $\begin{array}{l}\text { Width } \\
\text { [min] }\end{array}$ & $\begin{array}{c}\text { Area } \\
{\left[\mathrm{mAU}^{*} \mathrm{~s}\right]}\end{array}$ & $\begin{array}{l}\text { Height } \\
\text { [mAU] }\end{array}$ & $\begin{array}{c}\text { Area } \\
\%\end{array}$ \\
\hline 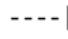 & & & & - & $\ldots$ & - \\
\hline 1 & 12.145 & VB & 0.3320 & 7520.88916 & 345.47867 & 49.9976 \\
\hline 2 & 14.839 & BB & 0.4057 & 7521.61328 & 281.50827 & 50.0024 \\
\hline
\end{tabular}

Totals :

$1.50425 \mathrm{e} 4 \quad 626.98694$ 
Data File D: \DATA \LSL\LSL-3-196\LSL-3-196 2019-01-20 14-29-19\058-1001.D Sample Name: LSL-3-196-6

\begin{tabular}{|c|c|c|}
\hline Acq. Operator & : & Seq. Line : 10 \\
\hline Acq. Instrument & : Instrument 2 & Location : Vial 58 \\
\hline Injection Date & : 1/20/2019 7:39:39 PM & $\begin{array}{rlc}\text { Inj } & : & 1 \\
\text { Inj Volume } & : & 5.000 \mu\end{array}$ \\
\hline
\end{tabular}

Acq. Method : D: \DATA \LSL\LSL-3-196\LSL-3-196 2019-01-20 14-29-19\DAD-OD(1-2)-98-2-1ML-

$\begin{aligned} & 5 U L-A L L-40 M I N . M \\ & \text { Last changed } \quad: \quad 9 / 21 / 2018 \text { 9:52:07 AM }\end{aligned}$

Analysis Method : D: \METHOD\LG

Last changed : 1/21/2019 10:00:12 PM

(modified after loading)

Additional Info : Peak(s) manually integrated

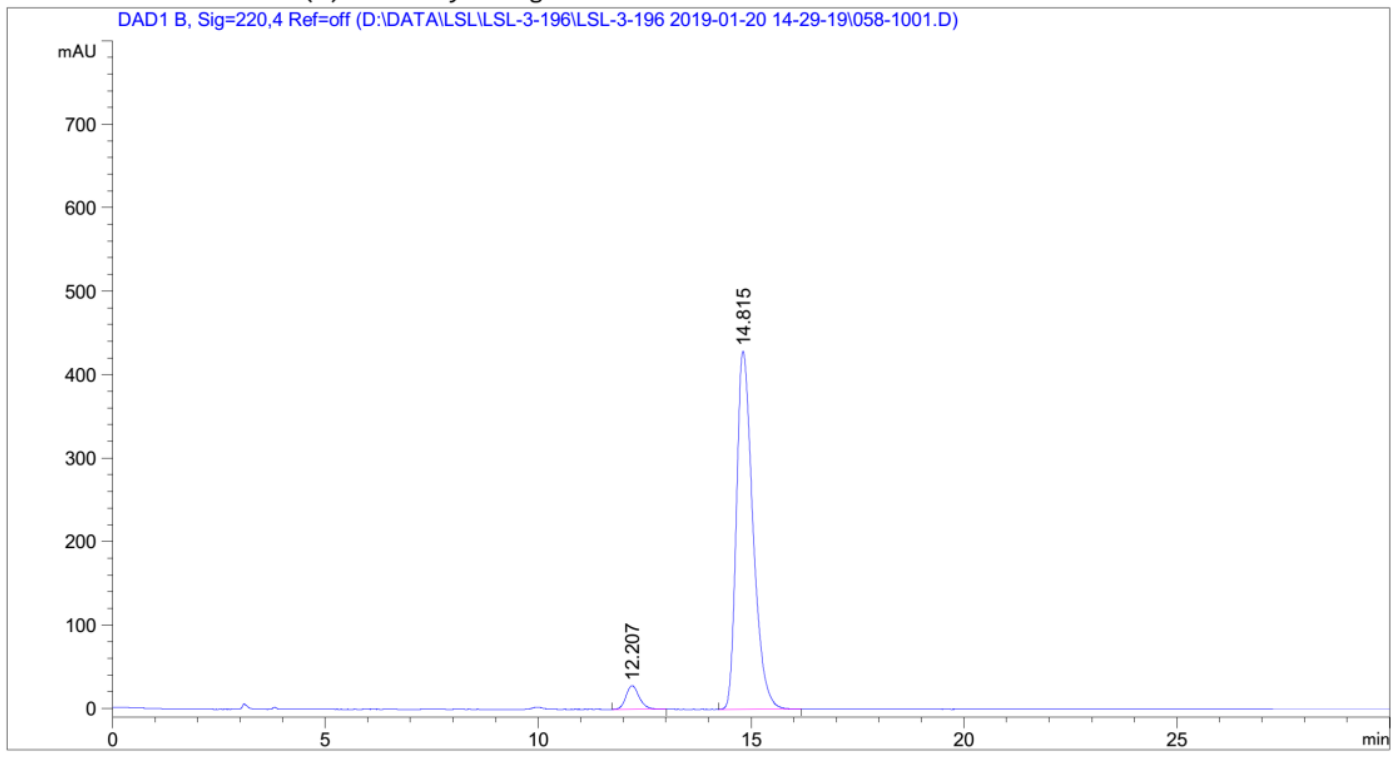

Area Percent Report

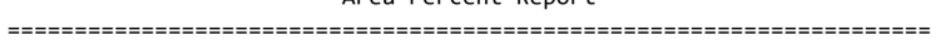

$\begin{array}{lll}\text { Sorted By } & : & \text { Signal } \\ \text { Multiplier } & : & 1.0000 \\ \text { Dilution } & : & 1.0000\end{array}$

Use Multiplier \& Dilution Factor with ISTDs

Signal 1: DAD1 B, Sig=220,4 Ref=off

\begin{tabular}{|c|c|c|c|c|c|}
\hline $\begin{array}{c}\text { Peak } \\
\#\end{array}$ & $\begin{array}{l}\text { RetTime Type } \\
\text { [min] }\end{array}$ & $\begin{array}{l}\text { Width } \\
\text { [min] }\end{array}$ & $\begin{array}{c}\text { Area } \\
{[\mathrm{mAU} * \mathrm{~s}]}\end{array}$ & $\begin{array}{l}\text { Height } \\
{[\mathrm{mAU}]}\end{array}$ & $\begin{array}{c}\text { Area } \\
\%\end{array}$ \\
\hline 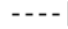 & |-...-. & & - & 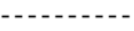 & 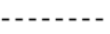 \\
\hline 1 & 12.207 BB & 0.3225 & 611.11481 & 28.23827 & 5.0254 \\
\hline 2 & $14.815 \mathrm{BB}$ & 0.4101 & $1.15495 \mathrm{e} 4$ & 429.02994 & 94.9746 \\
\hline Total & & & $1.21606 \mathrm{e} 4$ & 457.26821 & \\
\hline
\end{tabular}


<smiles>CCOC(=O)C(CC=O)Cc1ccc(C(C)(C)C)cc1</smiles>

Data File D: \DATA \XZC \ZZC-DATA-5\XZC-20190418-1 2019-04-18 19-55-56\065-1301.D Sample Name: LSL-tBu rac

$\begin{array}{lr}\text { Acq. Operator : } & \text { Seq. Line : } 13 \\ \text { Acq. Instrument : Instrument } 2 & \text { Location : Vial } 65 \\ \text { Injection Date : 4/19/2019 1:07:18 AM } & \text { Inj : } 1\end{array}$

Acq. Method : D: \DATA \XZC\XZC-DATA-5 \XZC-20190418-1 2019-04-18 19-55-56\DAD-OD(1-2)-95-51ML-5UL-ALL-60MIN.M

Last changed : 6/21/2018 2:29:29 PM

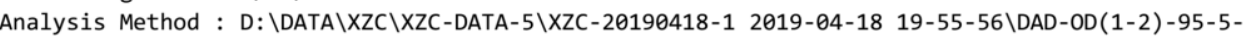
1ML-5UL-ALL-60MIN.M (Sequence Method)

Last changed : 4/19/2019 10:31:52 AM (modified after loading)

Additional Info : Peak(s) manually integrated

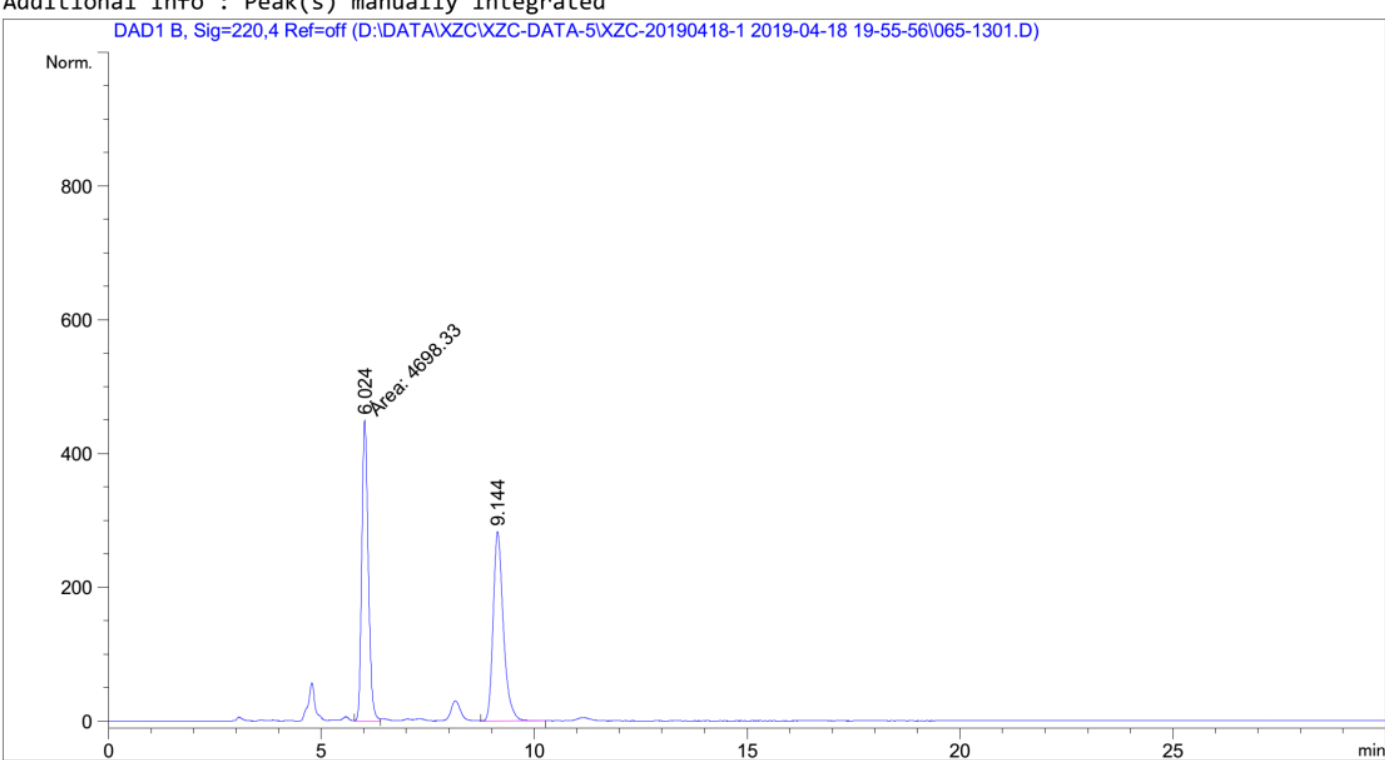

Area Percent Report

\begin{tabular}{|c|c|c|}
\hline Sorted By & : & Signal \\
\hline Multiplier & : & 1.0000 \\
\hline Dilution & : & 1.0000 \\
\hline
\end{tabular}

Use Multiplier \& Dilution Factor with ISTDs

Signal 1: DAD1 B, Sig $=220,4$ Ref=off

\begin{tabular}{cccccc}
$\begin{array}{c}\text { Peak RetTime Type } \\
\text { \# } \\
{[\mathrm{min}]}\end{array}$ & $\begin{array}{c}\text { Width } \\
{[\mathrm{min}]}\end{array}$ & $\begin{array}{c}\text { Area } \\
{[\mathrm{mAU} \text { s }]}\end{array}$ & $\begin{array}{c}\text { Height } \\
{[\mathrm{mAU}]}\end{array}$ & $\begin{array}{c}\text { Area } \\
\%\end{array}$ \\
\hdashline 1 & $6.024 \mathrm{MF}$ & 0.1740 & 4698.32861 & 449.96259 & 49.8533 \\
2 & $9.144 \mathrm{BB}$ & 0.2527 & 4725.97656 & 283.19824 & 50.1467 \\
& & & & & \\
Totals : & & 9424.30518 & 733.16083 &
\end{tabular}


Data File D: \DATA \XZC \XZC-DATA-5\XZC-20190418-1 2019-04-18 19-55-56\066-1401.D Sample Name: LSL-tBu

Acq. Operator

Acq. Instrument : Instrument 2

Injection Date : 4/19/2019 2:08:21 AM

Acq. Method D: $\backslash D A T A \backslash X Z C \backslash X Z C-D A T A$
$1 M L-5 U L-A L L-60 M I N . M$

Last changed : 6/21/2018 2:29:29 PM

Analysis Method : D: \DATA \XZC \XZC-DATA-5 \XZC-20190418-1 2019-04-18 19-55-56\DAD-OD(1-2)-95-51ML-5UL-ALL-60MIN.M (Sequence Method)

Last changed

$$
\begin{aligned}
& \text { : 4/19/2019 10:33:29 AM } \\
& \text { (modified after loading) }
\end{aligned}
$$

Additional Info : Peak(s) manually integrated

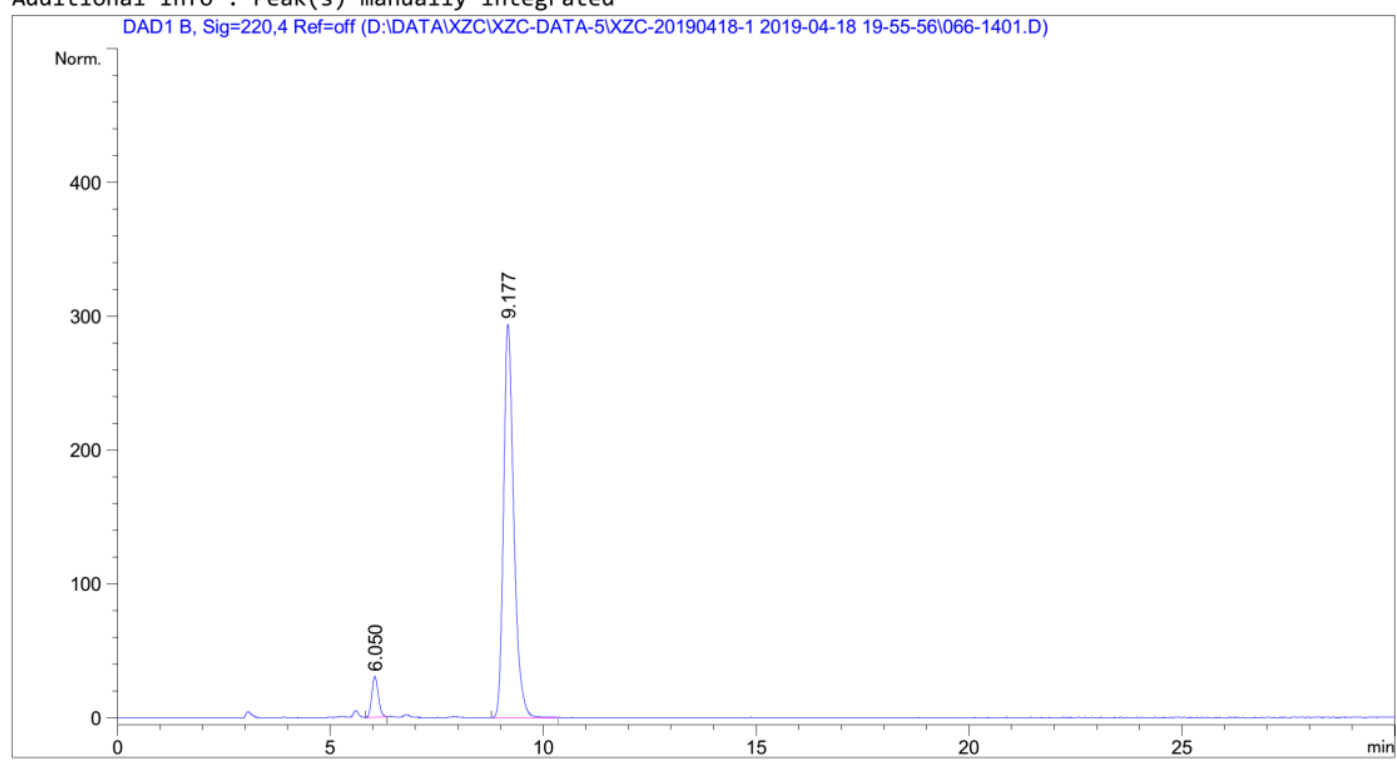

Area Percent Report

$\begin{array}{lll}================================ \\ \text { Sorted By } & : & \text { Signal } \\ \text { Multiplier } & : & 1.0000 \\ \text { Dilution } & : & 1.0000\end{array}$

Use Multiplier \& Dilution Factor with ISTDs

Signal 1: DAD1 B, Sig=220,4 Ref=off

\begin{tabular}{cccccc}
$\begin{array}{c}\text { Peak RetTime Type Width } \\
\text { \# }\end{array}$ [min] & $\begin{array}{c}\text { Area } \\
{[\mathrm{min}]}\end{array}$ & $\begin{array}{c}\text { Height } \\
{[\mathrm{mAU} \text { s }]}\end{array}$ & \multicolumn{1}{c}{$\begin{array}{c}\text { Area } \\
{[\mathrm{mAU}]}\end{array}$} & \multicolumn{1}{c}{$\%$} \\
\hline 1 & 6.050 BB & 0.1587 & 313.38785 & 30.30317 & 5.9826 \\
2 & 9.177 BB & 0.2557 & 4924.97021 & 293.62207 & 94.0174
\end{tabular}

Totals :

$5238.35806 \quad 323.92525$

Seq. Line : 14

Location : Vial 66

Inj : 1

Inj Volume : $5.000 \mu \mathrm{l}$

20190418-1 2019-04-18 19-55-56 \DAD-OD(1-2) -95-5- 
<smiles>CCOC(=O)C(CC=O)Cc1ccc(C#N)cc1</smiles>

Data File D: \DATA \GUAN YUQING \LJ-0530-WW \WW-4-128-1 2019-05-30 09-35-35\053-1301.D Sample Name: LSL-4-P-CN RAC
Acq. Operator :
Seq. Line : 13
Acq. Instrument : Instrument 2
Location : Vial 53
Injection Date : 5/30/2019 3:21:06 PM
Inj : 1
Inj Volume : $5.000 \mu 1$

Acq. Method : D: \DATA \GUAN YUQING \LJ-0530-WW \WW-4-128-1 2019-05-30 09-35-35\DAD-AD(1-2)90-10-1ML-5UL-ALL-40MIN.M

Last changed : 5/30/2019 10:39:11 AM

Analysis Method: D: \METHOD \GUAN YUQING \DAD-AD(1-2)-99-1-0.5ML-5UL-ALL-60MIN.M

Last changed : 5/31/2019 10:24:46 AM

(modified after loading)

Additional Info : Peak(s) manually integrated

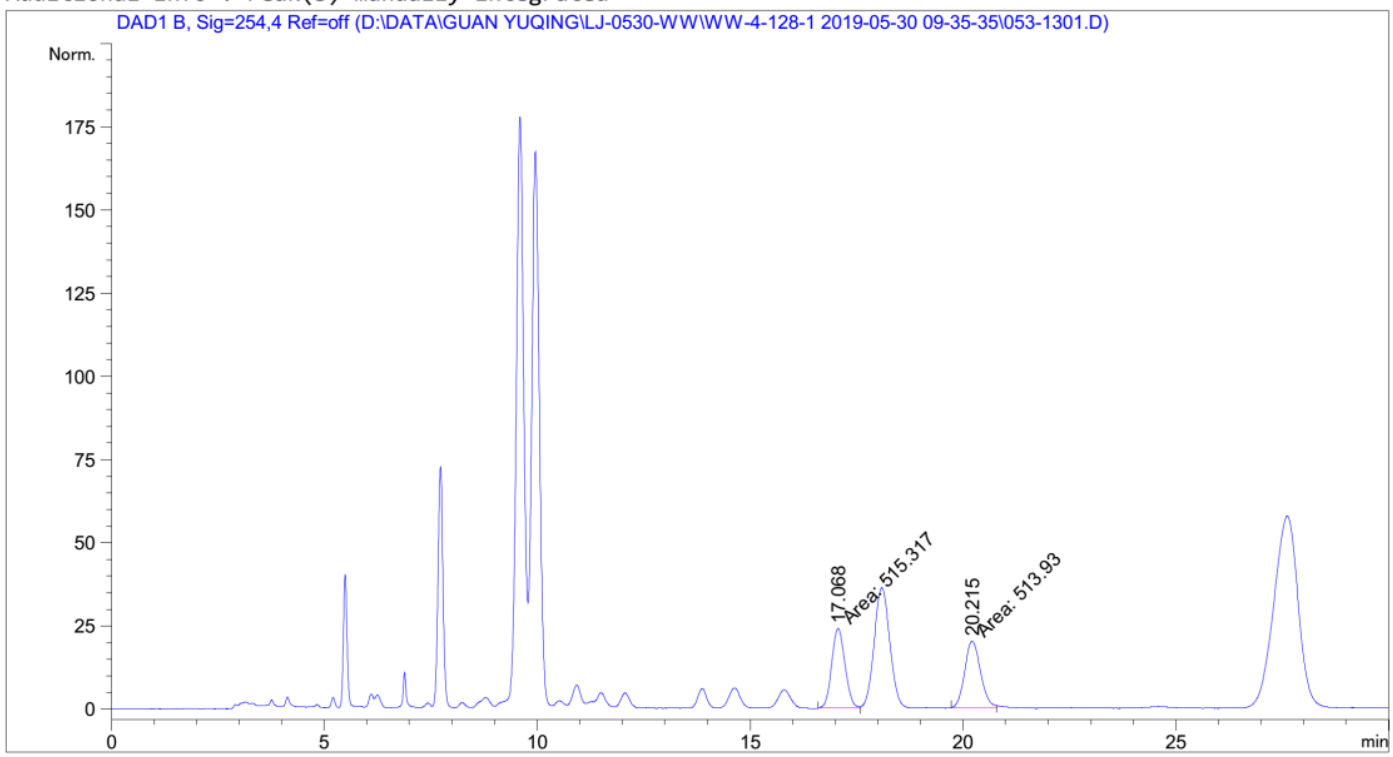

Area Percent Report

$\begin{array}{lll}================================= \\ \text { Sorted By } & : & \text { Signal } \\ \text { Multiplier } & : & 1.0000 \\ \text { Dilution } & : & 1.0000\end{array}$

Use Multiplier \& Dilution Factor with ISTDs

Signal 1: DAD1 B, Sig=254,4 Ref=off

\begin{tabular}{cccccc}
$\begin{array}{c}\text { Peak RetTime Type } \\
\text { W } \\
{[\mathrm{min}]}\end{array}$ & $\begin{array}{c}\text { Width } \\
{[\mathrm{min}]}\end{array}$ & $\begin{array}{c}\text { Area } \\
{[\mathrm{mAU} \text { s }]}\end{array}$ & $\begin{array}{c}\text { Height } \\
{[\mathrm{mAU}]}\end{array}$ & $\begin{array}{c}\text { Area } \\
\%\end{array}$ \\
\hline 1 & $17.068 \mathrm{MM}$ & 0.3594 & 515.31714 & 23.90012 & 50.0674 \\
2 & 20.215 MM & 0.4295 & 513.93018 & 19.94221 & 49.9326 \\
& & & & & \\
Totals : & & 1029.24731 & 43.84233
\end{tabular}




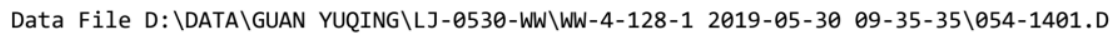
Sample Name: LSL-4-P-CN

Acq. Operator

Acq. Instrument : Instrument 2

Injection Date : 5/30/2019 4:02:10 PM

Acq. Method

Inj Volume : $5.000 \mu l$
: D: \DATA \GUAN YUQING \LJ-0530-WW $W W-4-128-1 \quad 2019-05-30$

Seq. Line : 14

Location : Vial 54

Inj : 1

90-10-1ML-5UL-ALL-40MIN.M

Last changed : 5/30/2019 10:39:11 AM

Analysis Method : D: \METHOD \GUAN YUQING DAD-AD(1-2)-99-1-0.5ML-5UL-ALL-60MIN.M

Last changed

5/31/2019 10:30:14 AM

(modified after loading)

Additional Info : Peak(s) manually integrated

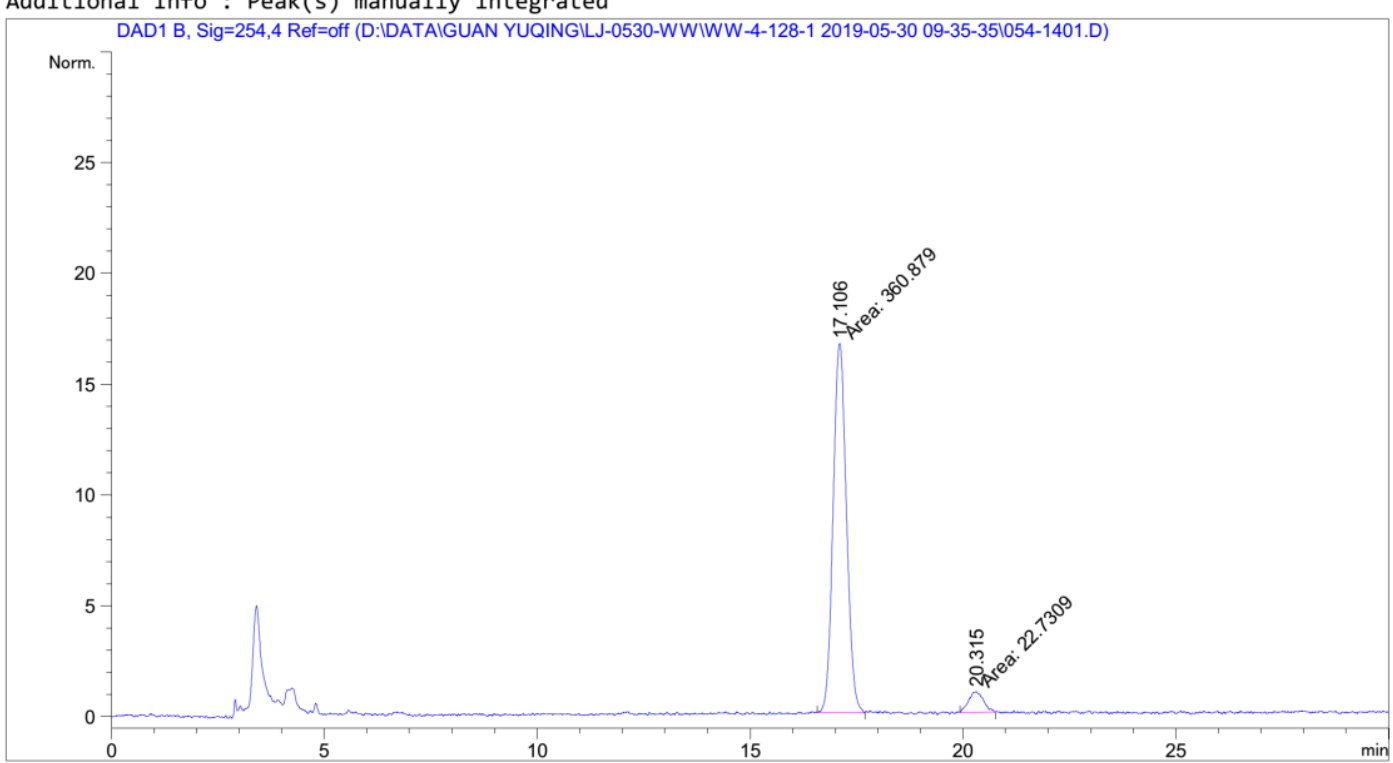

Area Percent Report

\begin{tabular}{|c|c|c|}
\hline Sorted By & $:$ & Signal \\
\hline Multiplier & $:$ & 1.0000 \\
\hline Dilution & & \\
\hline
\end{tabular}

Dilution

1.0000

Use Multiplier \& Dilution Factor with ISTDs

Signal 1: DAD1 B, Sig $=254,4$ Ref=off

\begin{tabular}{|c|c|c|c|c|c|}
\hline $\begin{array}{c}\text { Peak } \\
\#\end{array}$ & $\begin{array}{l}\text { RetTime Type } \\
\text { [min] }\end{array}$ & $\begin{array}{l}\text { Width } \\
\text { [min] }\end{array}$ & $\begin{array}{c}\text { Area } \\
{\left[\mathrm{mAU}^{*} \mathrm{~s}\right]}\end{array}$ & $\begin{array}{l}\text { Height } \\
\text { [mAU] }\end{array}$ & $\begin{array}{c}\text { Area } \\
\quad \%\end{array}$ \\
\hline-- & 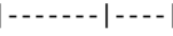 & & | & - & $\mid$ \\
\hline 1 & $17.106 \mathrm{MM}$ & 0.3612 & 360.87930 & 16.65151 & 94.0745 \\
\hline 2 & $20.315 \mathrm{MM}$ & 0.4038 & 22.73090 & $9.38171 \mathrm{e}-1$ & 5.9255 \\
\hline \multicolumn{3}{|c|}{ Totals : } & 383.61020 & 17.58968 & \\
\hline
\end{tabular}

Instrument 2 5/31/2019 10:30:20 AM

Page 1 of 2 


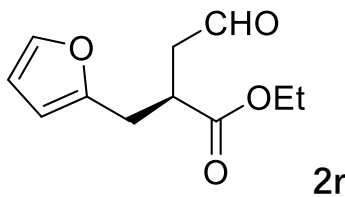

Data File D: \DATA \LWD\LWD-5-60\LWD-5-60-14 2019-05-06 09-32-49\033-2401.D Sample Name: funan rac-

$\begin{array}{lr}\text { Acq. Operator : } & \text { Seq. Line : } 24 \\ \text { Acq. Instrument : Instrument } 2 & \text { Location : Vial } 33 \\ \text { Injection Date : 5/6/2019 10:58:28 PM } & \text { Inj : } 1\end{array}$

Acq. Method : D: \DATA \LWD\LWD-5-60\LWD-5-60-14 2019-05-06 09-32-49\DAD-OD(1-2)-98-2-1ML5UL-ALL-40MIN.M

Last changed : 5/6/2019 9:51:19 PM

(modified after loading)

Analysis Method : D: \DATA \LWD LWD-5-60\LWD-5-60-14 2019-05-06 09-32-49\DAD-OD(1-2)-98-2-1ML5UL-ALL-40MIN.M (Sequence Method)

Last changed : 5/7/2019 9:27:45 AM

(modified after loading)

Additional Info : Peak(s) manually integrated

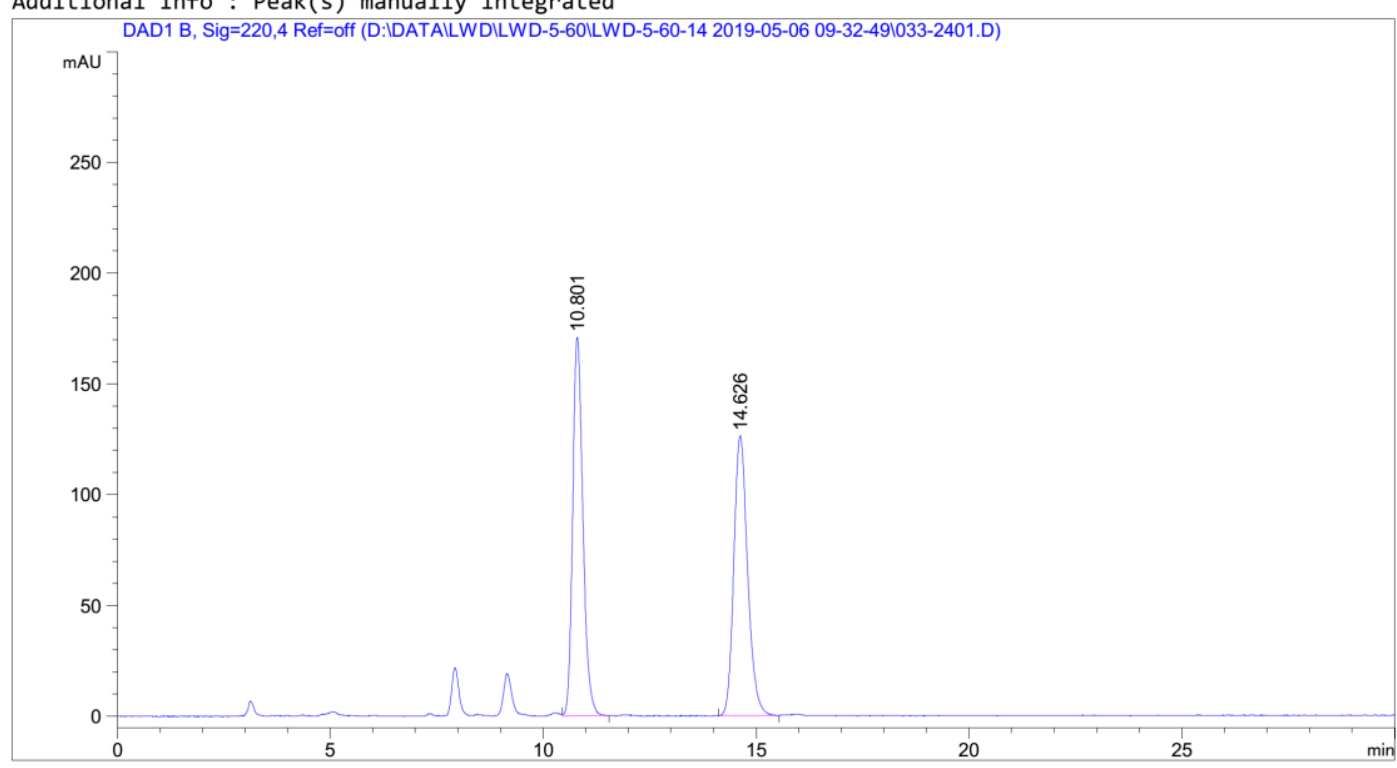

Area Percent Report

Sorted By : Signal

Multiplier $\quad$ : 1.0000

Dilution : 1.0000

Use Multiplier \& Dilution Factor with ISTDs

Signal 1: DAD1 B, Sig=220,4 Ref=off

\begin{tabular}{|c|c|c|c|c|c|}
\hline $\begin{array}{c}\text { Peak } \\
\#\end{array}$ & $\begin{array}{l}\text { RetTime Type } \\
\text { [min] }\end{array}$ & $\begin{array}{l}\text { Width } \\
\text { [min] }\end{array}$ & $\begin{array}{c}\text { Area } \\
{[\mathrm{mAU} * \mathrm{~s}]}\end{array}$ & $\begin{array}{l}\text { Height } \\
\text { [mAU] }\end{array}$ & $\begin{array}{c}\text { Area } \\
\%\end{array}$ \\
\hline & $-\ldots-1 \mid-$ & & | & 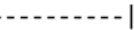 & 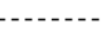 \\
\hline 1 & $10.801 \mathrm{VB}$ & 0.2541 & 2842.55835 & 170.88455 & 49.9906 \\
\hline 2 & $14.626 \mathrm{BB}$ & 0.3407 & 2843.62280 & 126.29852 & 50.0094 \\
\hline Total & Is: & & 5686.18115 & 297.18307 & \\
\hline
\end{tabular}


Data File D: \DATA \LWD\LWD-5-60\LWD-5-60-14 2019-05-06 09-32-49\034-2501.D Sample Name: funan

Acq. Operator

Acq. Instrument : Instrument 2

Injection Date : 5/6/2019 11:49:27 PM

Seq. Line : 25

Location : Vial 34

Inj : 1

Inj Volume : $5.000 \mu \mathrm{l}$

Acq. Method : D: \DATA \LWD \LWD-5-60\LWD-5-60-14 2019-05-06 09-32-49\DAD-OD(1-2)-98-2-1ML5UL-ALL-40MIN.M

Last changed : 5/6/2019 9:51:19 PM (modified after loading)

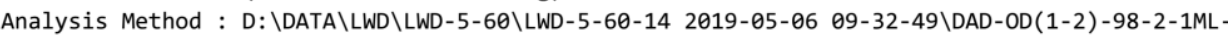
5UL-ALL-40MIN.M (Sequence Method)

Last changed : 5/7/2019 9:30:14 AM (modified after loading)

Additional Info : Peak(s) manually integrated

D1 B, Sig=220,4 Ref=off (D:IDATAILWDILWD-5-60ILWD-5-60-14 2019-05-06 09-32-491034-2501.D)

mAU

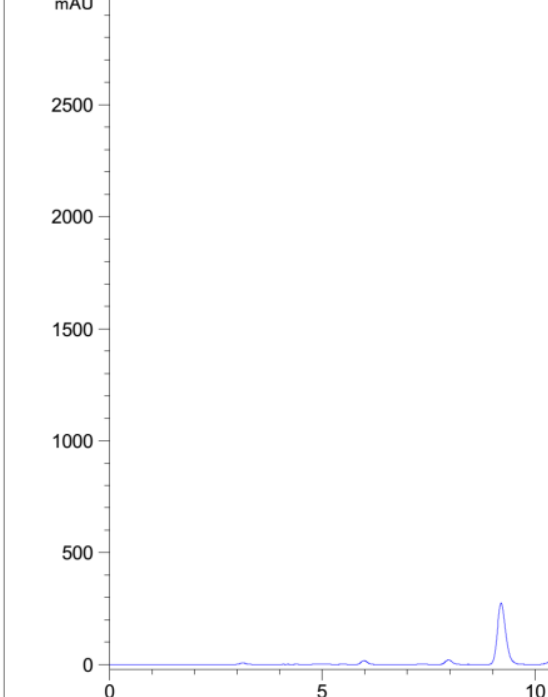

Area Percent Report

\begin{tabular}{|c|c|c|}
\hline Sorted By & $:$ & Signal \\
\hline Multiplier & $:$ & 1.0000 \\
\hline ilution & : & \\
\hline
\end{tabular}

: 1.0000

Use Multiplier \& Dilution Factor with ISTDs

Signal 1: DAD1 B, Sig=220,4 Ref=off

\begin{tabular}{|c|c|c|c|c|c|}
\hline $\begin{array}{c}\text { Peak } \\
\#\end{array}$ & $\begin{array}{l}\text { RetTime Type } \\
\text { [min] }\end{array}$ & $\begin{array}{l}\text { Width } \\
\text { [min] }\end{array}$ & $\begin{array}{c}\text { Area } \\
{[\mathrm{mAU} * \mathrm{~s}]}\end{array}$ & $\begin{array}{l}\text { Height } \\
\text { [mAU] }\end{array}$ & $\begin{array}{c}\text { Area } \\
\%\end{array}$ \\
\hline-- & $\mid-\ldots-1-1$ & - & | & | & | \\
\hline 1 & $10.869 \mathrm{MM}$ & 0.2743 & 2494.06567 & 151.53320 & 6.0264 \\
\hline 2 & $14.639 \mathrm{MM}$ & 0.4075 & $3.88914 \mathrm{e} 4$ & 1590.57520 & 93.9736 \\
\hline Tot & s: & & $4.13855 \mathrm{e} 4$ & 1742.10840 & \\
\hline
\end{tabular}

Instrument 2 5/7/2019 9:30:21 AM

Page 1 of 2 
<smiles>[R]OC(=O)[C@H](CC=O)CCOCc1ccccc1</smiles>

Data File D: \DATA \LWDLLD-5-60\LWD-5-60-14 2019-05-06 09-32-49\031-2101.D Sample Name: Bno rac-

$\begin{array}{lr}\text { Acq. Operator : } & \text { Seq. Line : } 21 \\ \text { Acq. Instrument : Instrument } 2 & \text { Location : Vial } 31 \\ \text { Injection Date : 5/6/2019 8:35:24 PM } & \text { Inj : } 1\end{array}$

Acq. Method : D: \DATA \LWD\LWD-5-60\LWD-5-60-14 2019-05-06 09-32-49\DAD-OD(1-2)-98-2-1ML5UL-ALL-4OMIN.M

Last changed : 9/21/2018 9:52:07 AM

Analysis Method : D: \DATA \LWD\LWD-5-60\LWD-5-60-14 2019-05-06 09-32-49\DAD-OD(1-2)-98-2-1ML5UL-ALL-40MIN.M (Sequence Method)

Last changed : 5/7/2019 9:22:30 AM (modified after loading)

Additional Info : Peak(s) manually integrated

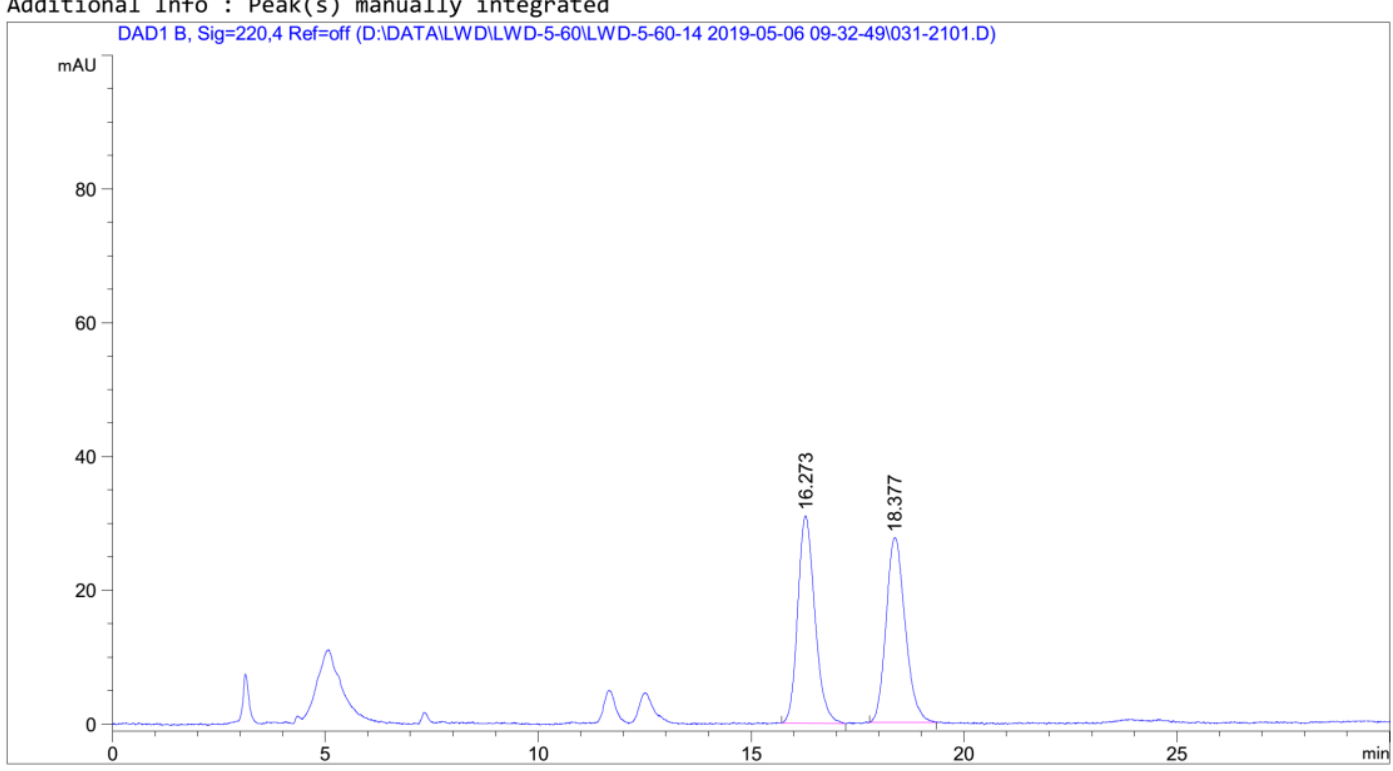

Area Percent Report

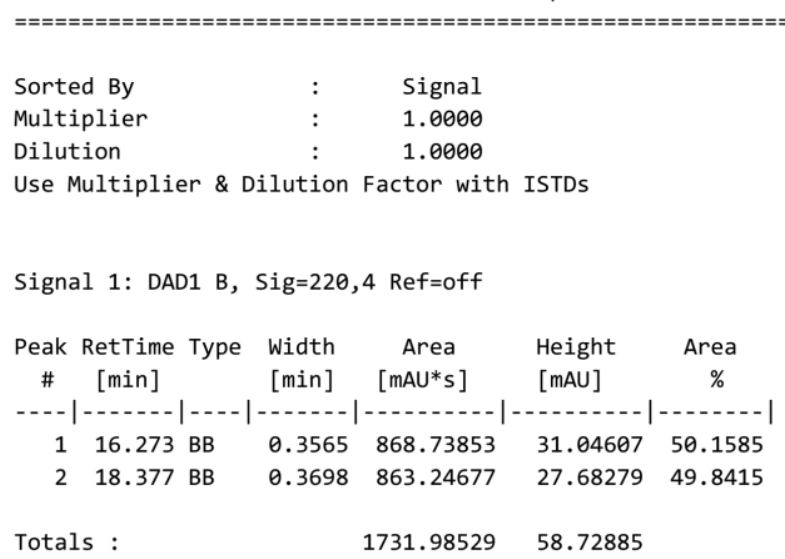


Data File D: \DATA \LWD\LWD-5-60\LWD-5-60-14 2019-05-06 09-32-49\032-2201.D Sample Name: Bno

\begin{tabular}{|c|c|c|}
\hline Acq. Operator & $:$ & Seq. Line : 22 \\
\hline Acq. Instrument & : Instrument 2 & Location : Vial 32 \\
\hline Injection Date & : 5/6/2019 9:16:25 PM & $\begin{array}{rlc}\text { Inj } & : & 1 \\
\text { Inj Volume } & : & 5.000 \mu\end{array}$ \\
\hline
\end{tabular}

Acq. Method : D: \DATA \LWD \LWD-5-60\LWD-5-60-14 2019-05-06 09-32-49\DAD-OD(1-2)-98-2-1ML5UL-ALL-40MIN.M

Last changed : 5/6/2019 9:51:19 PM (modified after loading)

Analysis Method : D: \DATA \LWD \LWD-5-60\LWD-5-60-14 2019-05-06 09-32-49\DAD-OD(1-2)-98-2-1ML5UL-ALL-40MIN.M (Sequence Method)

Last changed : 5/7/2019 9:24:41 AM (modified after loading)

Additional Info : Peak(s) manually integrated

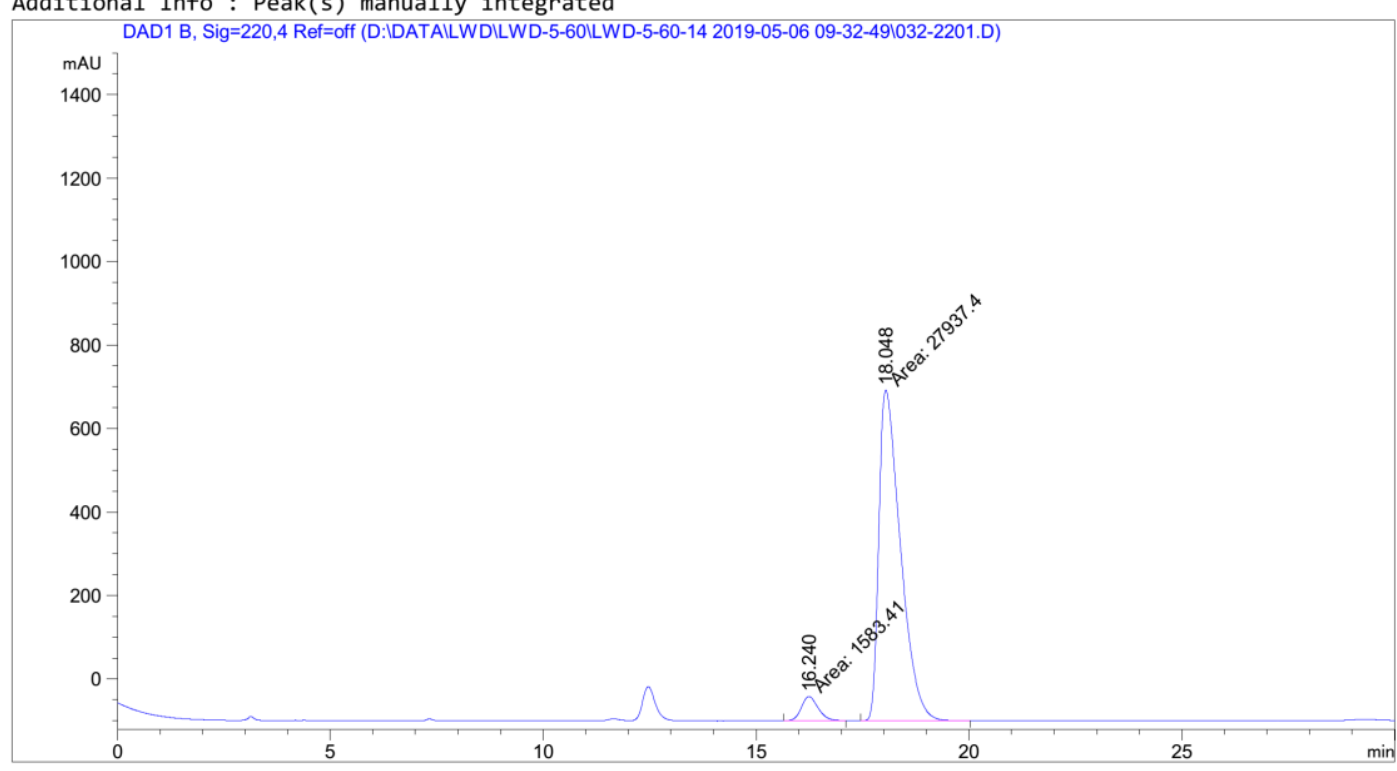

Area Percent Report

\begin{tabular}{|c|c|c|}
\hline Sorted By & $:$ & Signal \\
\hline Multiplier & : & 1.0000 \\
\hline Dilution & : & 1.0000 \\
\hline
\end{tabular}

Use Multiplier \& Dilution Factor with ISTDs

Signal 1: DAD1 B, Sig=220,4 Ref=off

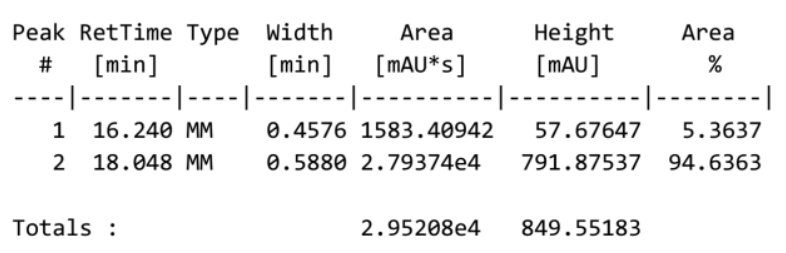




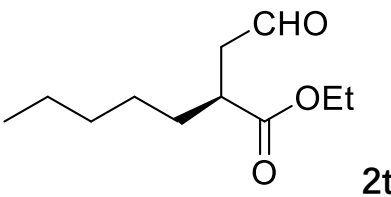

Data File D: \DATA\LGY \LGY-3-140\LGY-3-140 2019-04-17 08-20-32\063-3501.D Sample Name: LSL-he rac-
Acq. Operator :
Seq. Line : 35
Location : Vial 63
Acq. Instrument : Instrument 2
Inj : 1
Injection Date : 4/18/2019 12:37:17 AM
Inj Volume : $5.000 \mu 1$

Acq. Method : D: \DATA \LGY \LGY-3-140\LGY-3-140 2019-04-17 08-20-32\DAD-OD(1-2)-98-2-1ML-

5UL-ALL-4OMIN.M

Last changed : 9/21/2018 9:52:07 AM

Analysis Method: D: \DATA\LGY \LGY-3-140\LGY-3-140 2019-04-17 08-20-32 \DAD-OD(1-2)-98-2-1ML-

5UL-ALL-40MIN.M (Sequence Method)

Last changed : 4/18/2019 11:25:39 AM

(modified after loading)

Additional Info : Peak(s) manually integrated

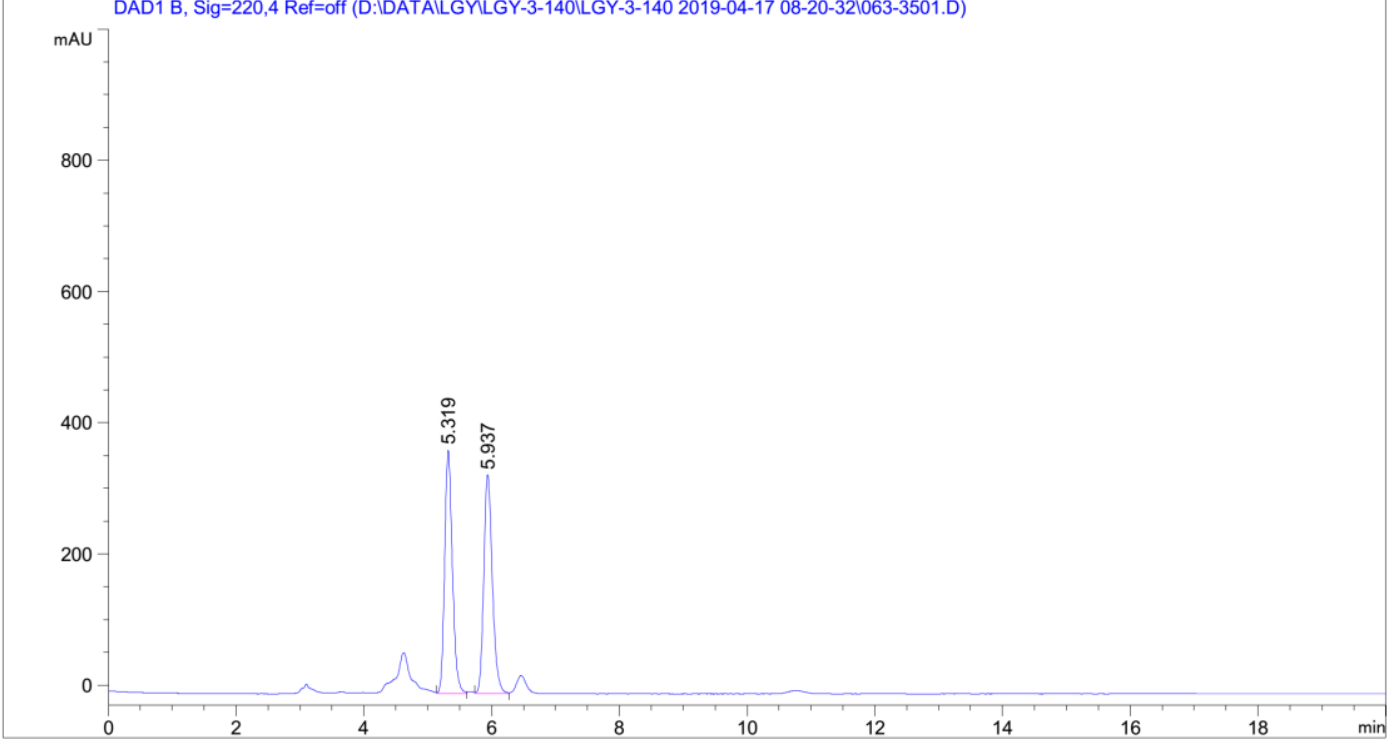

Area Percent Report

$\begin{array}{lll} & & \\ \text { Sorted By } & : & \text { Signal } \\ \text { Multiplier } & : & 1.0000 \\ \text { Dilution } & : & 1.0000\end{array}$

Use Multiplier \& Dilution Factor with ISTDS

Signal 1: DAD1 B, Sig=220,4 Ref=off

\begin{tabular}{cccccc}
$\begin{array}{c}\text { Peak RetTime Type } \\
\text { \# } \\
{[\text { min] }}\end{array}$ & $\begin{array}{c}\text { Width } \\
{[\mathrm{min}]}\end{array}$ & $\begin{array}{c}\text { Area } \\
{[\mathrm{mAU} \text { s }]}\end{array}$ & $\begin{array}{c}\text { Height } \\
{[\mathrm{mAU}]}\end{array}$ & $\begin{array}{c}\text { Area } \\
\%\end{array}$ \\
\hline 1 & $5.319 \mathrm{VV}$ & 0.1280 & 3114.99536 & 370.68976 & 50.0472 \\
2 & $5.937 \mathrm{WV}$ & 0.1428 & 3109.11621 & 333.35901 & 49.9528 \\
& & & & & \\
Totals : & & 6224.11157 & 704.04877 &
\end{tabular}


Data File D:\DATA \LS...-3-126 AND 127-1\LSL-3-126 AND 127-1 2018-10-13 19-57-46\002-0801.D Sample Name: LSL-3-127-1-2

\begin{tabular}{|c|c|c|}
\hline Acq. Operator & $:$ & Seq. Line : \\
\hline Acq. Instrument & : Instrument 2 & Location : Vial 2 \\
\hline Injection Date & : 10/13/2018 10:16:16 PM & 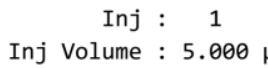 \\
\hline
\end{tabular}

Acq. Method : D: \DATA \LSL \LSL-3-126 AND 127-1\LSL-3-126 AND 127-1 2018-10-13 19-57-46\DAD $-O D(1-2)-98-2-1 M L-5 U L-A L L-40 M I N . M$

Last changed : 9/21/2018 9:52:07 AM

Analysis Method: D: \METHOD \GUAN YUQING \LONGJIAO\VWD-AS(1-6)-95-5-0.5ML-5UL-220NM-10MIN.M

Last changed : 4/18/2019 11:18:58 AM (modified after loading)

Additional Info : Peak(s) manually integrated

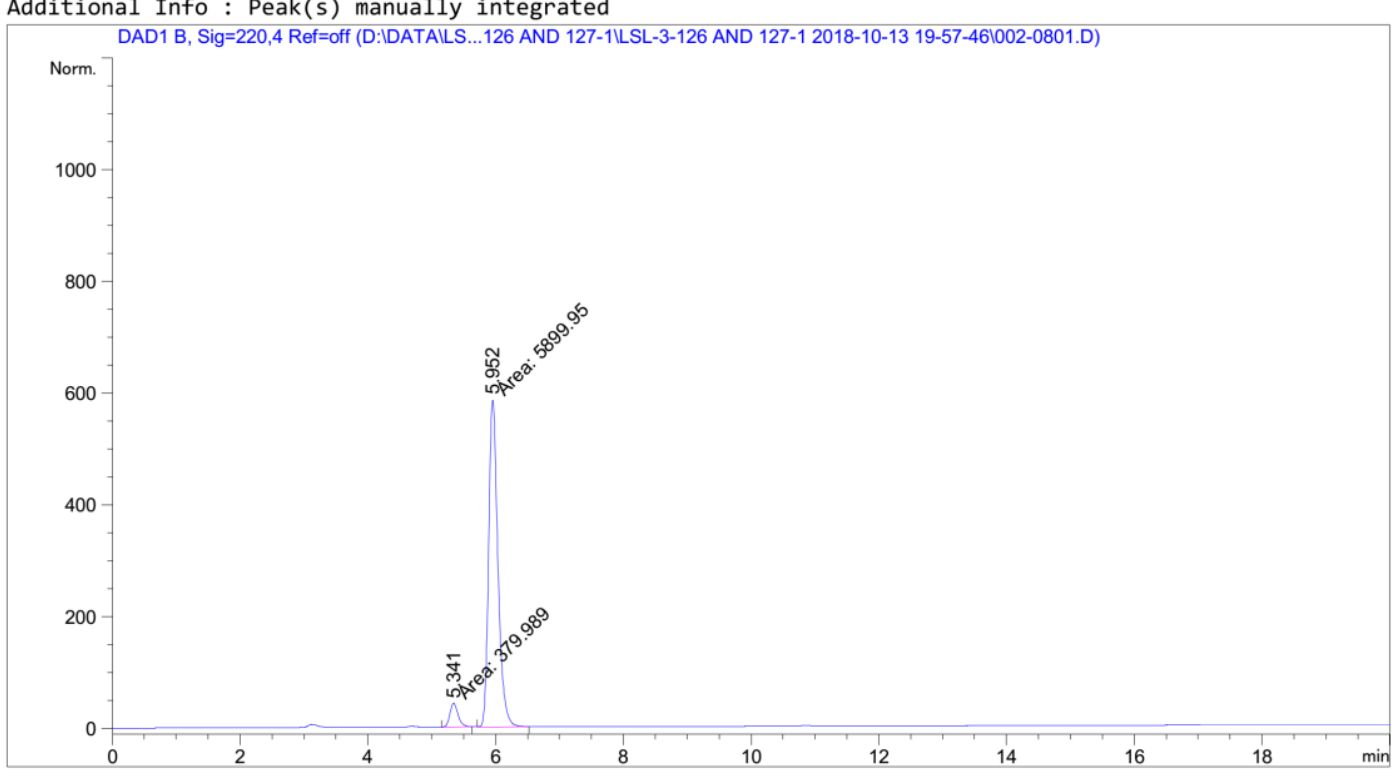

Area Percent Report

\begin{tabular}{|c|c|c|}
\hline Sorted By & : & Signal \\
\hline Multiplier & : & 1.0000 \\
\hline Dilution & : & 1.0000 \\
\hline
\end{tabular}

Use Multiplier \& Dilution Factor with ISTDS

Signal 1: DAD1 B, Sig $=220,4$ Ref $=0 f f$

\begin{tabular}{cccccc}
$\begin{array}{c}\text { Peak RetTime Type } \\
\text { \# } \\
{[\mathrm{min}]}\end{array}$ & $\begin{array}{c}\text { Width } \\
{[\mathrm{min}]}\end{array}$ & $\begin{array}{c}\text { Area } \\
{[\mathrm{mAU} \text { s }]}\end{array}$ & $\begin{array}{l}\text { Height } \\
{[\mathrm{mAU}]}\end{array}$ & \multicolumn{1}{c}{$\begin{array}{c}\text { Area } \\
\%\end{array}$} \\
\hline 1 & $5.341 \mathrm{MM}$ & 0.1480 & 379.98932 & 42.78594 & 6.0508 \\
2 & $5.952 \mathrm{MM}$ & 0.1679 & 5899.94971 & 585.57678 & 93.9492 \\
& & & & & \\
Totals : & & 6279.93903 & 628.36272 &
\end{tabular}




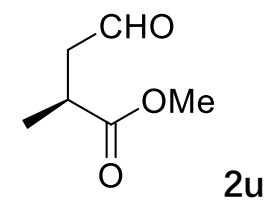

Data File D: \DATA \LSL\LSL-4-64\LSL-4-64 2019-06-01 08-40-49\031-0301.D Sample Name: LSL-4-64-1-RAC
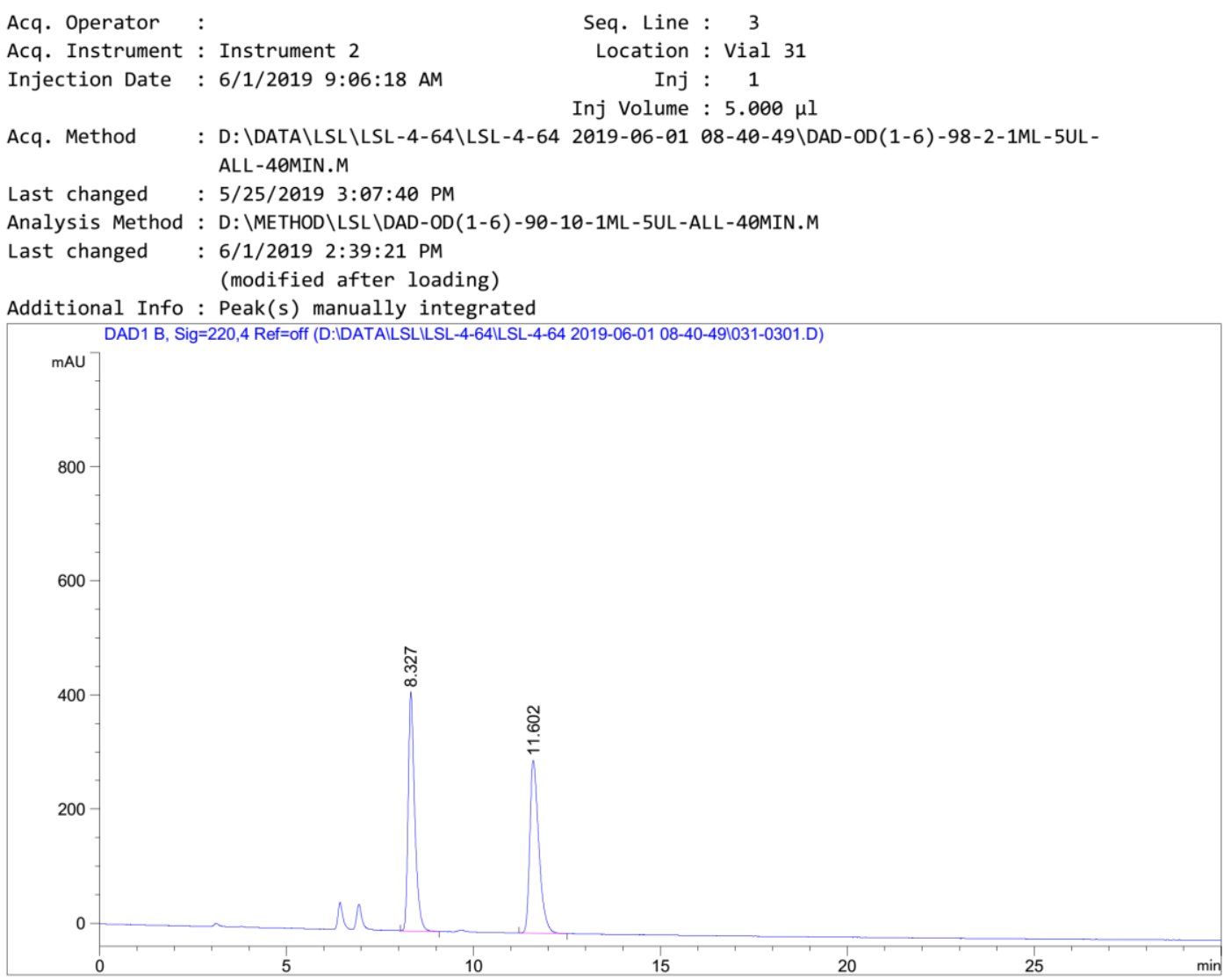

Area Percent Report

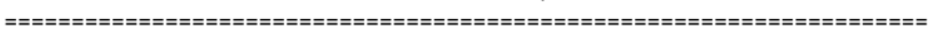

$\begin{array}{lll}\text { Sorted By } & : & \text { Signal } \\ \text { Multiplier } & : & 1.0000 \\ \text { Dilution } & : & 1.0000\end{array}$

Use Multiplier \& Dilution Factor with ISTDs

Signal 1: DAD1 B, Sig=220,4 Ref=off

\begin{tabular}{cccccc}
$\begin{array}{c}\text { Peak RetTime Type } \\
\text { Width } \\
\text { [min] }\end{array}$ & $\begin{array}{c}\text { Area } \\
{[\mathrm{min}]}\end{array}\left[\begin{array}{c}\text { HAU*s] } \\
{[\mathrm{mAU}]}\end{array}\right.$ & $\begin{array}{c}\text { Area } \\
\%\end{array}$ \\
\hline 1 & 8.327 BB & 0.1850 & 5131.59619 & 418.59070 & 50.1290 \\
2 & 11.602 BB & 0.2526 & 5105.17969 & 303.02502 & 49.8710
\end{tabular}

Totals : $\quad 1.02368 \mathrm{e} 4 \quad 721.61572$ 
Data File D: \DATA \LSLISL-4-64\LSL-4-64 2019-06-01 08-40-49\032-0401.D Sample Name: LSL-4-64-1

\begin{tabular}{|c|c|c|}
\hline Acq. Operator & $:$ & Seq. Line : 4 \\
\hline Acq. Instrument & : Instrument 2 & Location : Vial 32 \\
\hline Injection Date & : 6/1/2019 9:47:18 AM & 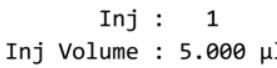 \\
\hline
\end{tabular}

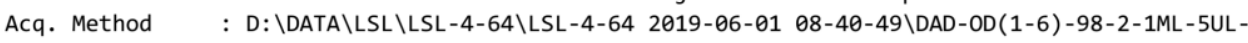
ALL-40MIN.M

Last changed : 5/25/2019 3:07:40 PM

Analysis Method : D: \METHOD\LSL \DAD-OD(1-6)-90-10-1ML-5UL-ALL-40MIN.M

Last changed : 6/1/2019 2:41:53 PM

(modified after loading)

Additional Info : Peak(s) manually integrated

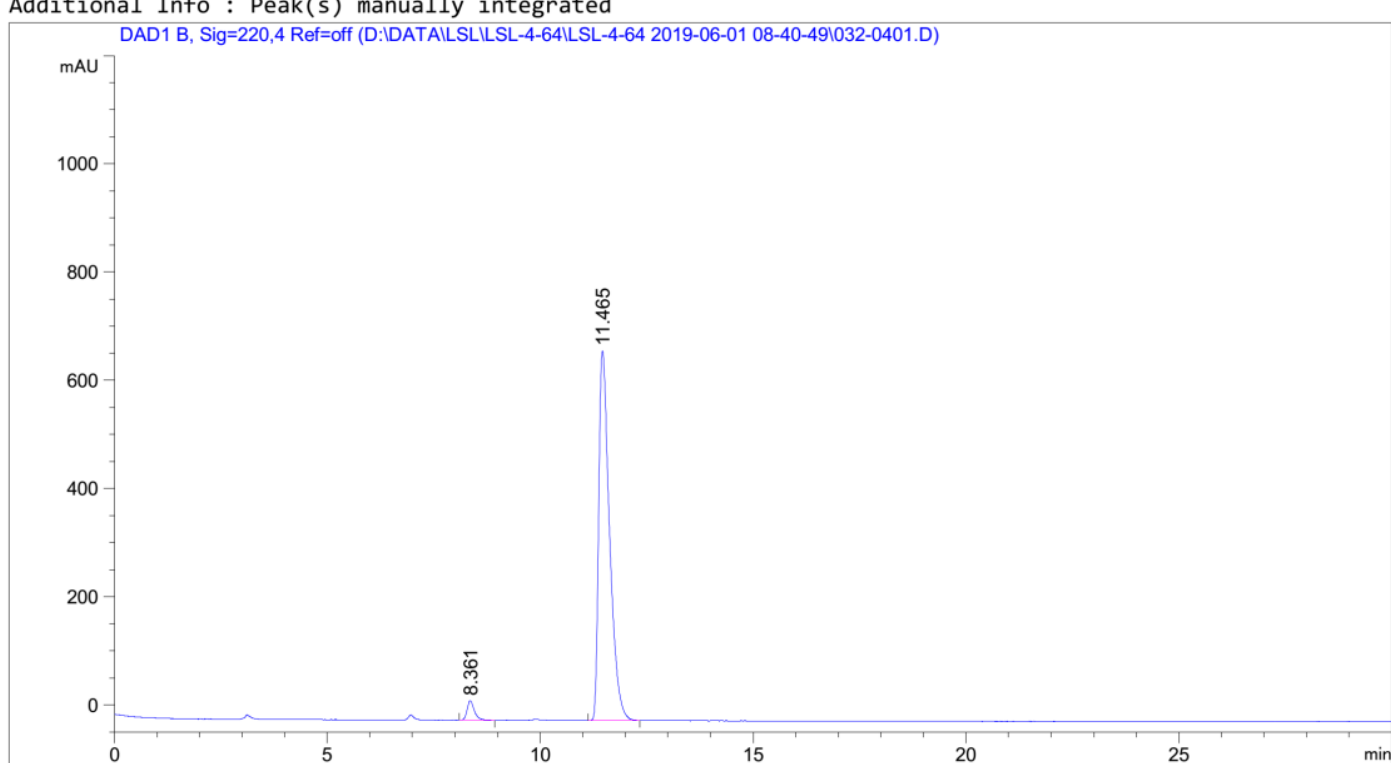

Area Percent Report

\begin{tabular}{|c|c|}
\hline Sorted By & \\
\hline Multiplier & : \\
\hline
\end{tabular}

Dilution : 1.0000

Use Multiplier \& Dilution Factor with ISTDs

Signal 1: DAD1 B, Sig=220,4 Ref=off

\begin{tabular}{|c|c|c|c|c|c|}
\hline $\begin{array}{c}\text { Peak } \\
\#\end{array}$ & $\begin{array}{l}\text { RetTime Type } \\
\text { [min] }\end{array}$ & $\begin{array}{l}\text { Width } \\
\text { [min] }\end{array}$ & $\begin{array}{c}\text { Area } \\
{\left[\mathrm{mAU}^{*} \mathrm{~s}\right]}\end{array}$ & $\begin{array}{l}\text { Height } \\
\text { [mAU] }\end{array}$ & $\begin{array}{c}\text { Area } \\
\%\end{array}$ \\
\hline 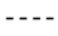 & $-\cdots$ & - & | & - & | \\
\hline 1 & $8.361 \mathrm{BB}$ & 0.1836 & 433.71613 & 36.25093 & 3.4675 \\
\hline 2 & $11.465 \mathrm{BB}$ & 0.2660 & $1.20743 \mathrm{e} 4$ & 683.69055 & 96.5325 \\
\hline Tota & 1s : & & $1.25080 \mathrm{e} 4$ & 719.94149 & \\
\hline
\end{tabular}


<smiles>COC(=O)CC(CC=O)C(=O)OC</smiles>

Data File D: \DATA LLSLILSL-4-64\LSL-4-64-2 2019-06-01 14-57-08\033-0301.D Sample Name: LSL-4-64-3-RAC
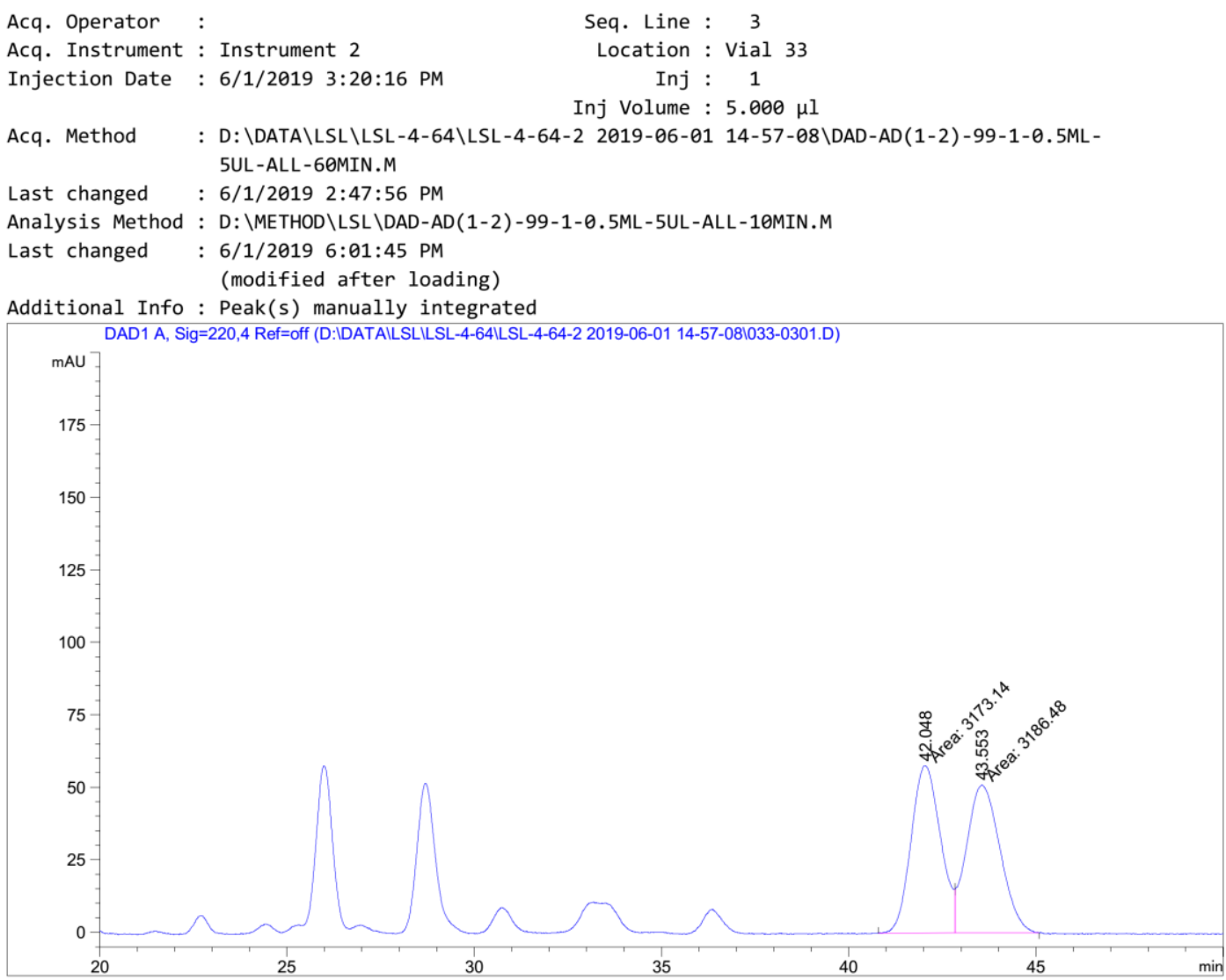

Area Percent Report

\begin{tabular}{|c|c|c|}
\hline Sorted By & : & Signal \\
\hline Multiplier & : & 1.0000 \\
\hline Dilution & : & 1.0000 \\
\hline
\end{tabular}

Use Multiplier \& Dilution Factor with ISTDs

Signal 1: DAD1 A, Sig=220,4 Ref=off

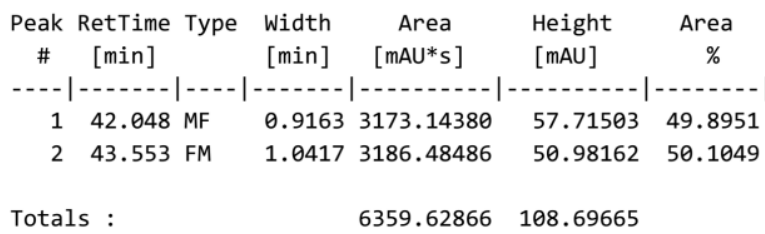




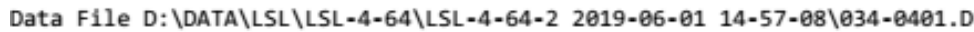
Sample Name: LSL-4-64-3

$\begin{array}{lr}\text { Acq. Operator : } & \text { Seq. Line : } 4 \\ \text { Acq. Instrument : Instrument } 2 & \text { Location : Vial } 34 \\ \text { Injection Date : } 6 / 1 / 20194: 21: 16 \mathrm{PM} & \text { Inj : } 1 \\ & \text { Inj Volume : } 5.600 \mu 1\end{array}$

Acq. Method : D: \DATA SUL-ALL-60MIN. M

Last changed : 6/1/2019 2:47:56 PM

Analysis Method: D: MMETHOD \LSL \DAD-AD(1-2)-99-1-0.5ML-5UL-ALL-10MIN.M

Last changed : 6/1/2019 6:04:36 PM

(modified after loading)

Additional Info: Peak(s) manually integrated

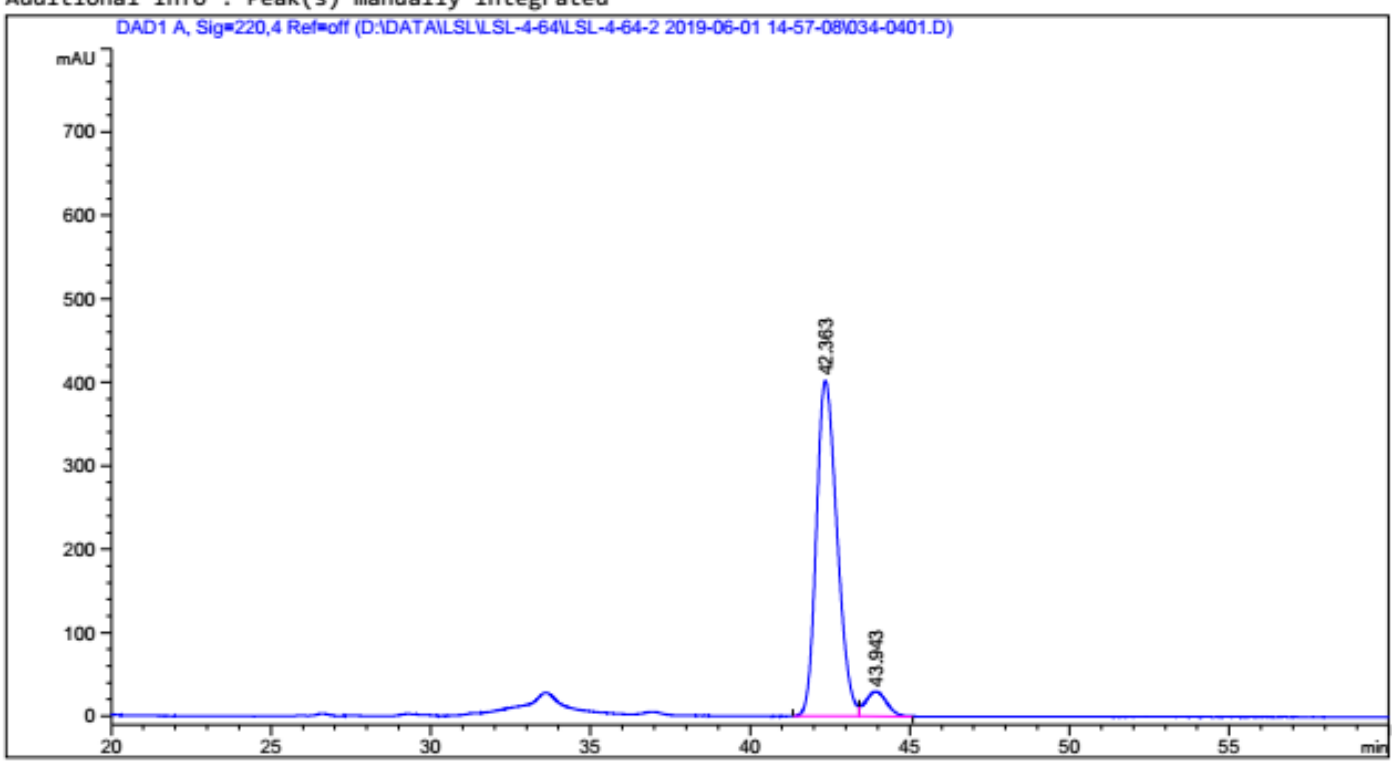

Area Percent Report

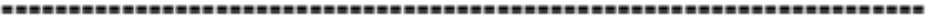

$\begin{array}{lll}\text { Sorted By } & : & \text { Signal } \\ \text { Multiplier } & : & 1.0000 \\ \text { Dilution } & : & 1.0000\end{array}$

Use Multiplier \& Dilution Factor with ISTDs

Signal 1: DAD1 A, Sig=220,4 Ref=off

\begin{tabular}{|c|c|c|c|c|c|}
\hline $\begin{array}{c}\text { Peak } \\
\#\end{array}$ & $\begin{array}{l}\text { RetTime Type } \\
\text { [min] }\end{array}$ & $\begin{array}{l}\text { Width } \\
\text { [min] }\end{array}$ & $\begin{array}{c}\text { Area } \\
{\left[\mathrm{mAU}^{*} \mathrm{~s}\right]}\end{array}$ & $\begin{array}{l}\text { Height } \\
\text { [maU] }\end{array}$ & $\begin{array}{c}\text { Area } \\
\%\end{array}$ \\
\hline$\cdots$ & - & & $\left|\begin{array}{lll}n & \ldots\end{array}\right|$ & . & ......... \\
\hline 1 & $42.363 \mathrm{BV}$ & 0.6521 & $1.81315 \mathrm{e} 4$ & 402.07605 & 93.0210 \\
\hline 2 & $43.943 \mathrm{VB}$ & 0.5456 & 1360.34131 & 29.85603 & 6.9790 \\
\hline Total & Is : & & $1.94918 \mathrm{e} 4$ & 431.93208 & \\
\hline
\end{tabular}




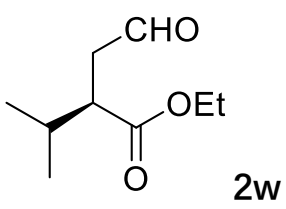




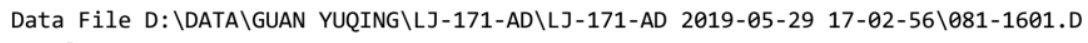
Sample Name: LSL-4-61-rac

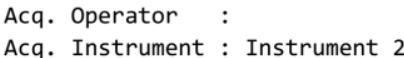
Seq. Line : 16
Injection Date : 5/29/2019 10:52:16 PM
Inj : 1
Inj Volume : $5.000 \mu \mathrm{l}$

Acq. Method : D: \DATA \GUAN YUQING \LJ-171-AD \LJ-171-AD 2019-05-29 17-02-56\DAD-OD(1-6)-982-1ML-5UL-ALL-40MIN.M

Last changed : 5/25/2019 3:07:40 PM

Analysis Method: D: \METHOD\LWD\DAD-AD(1-6)-95-5-1ML-3UL-ALL-30MIN-0813.M

Last changed : 8/13/2019 8:09:00 PM

(modified after loading)

Additional Info : Peak(s) manually integrated

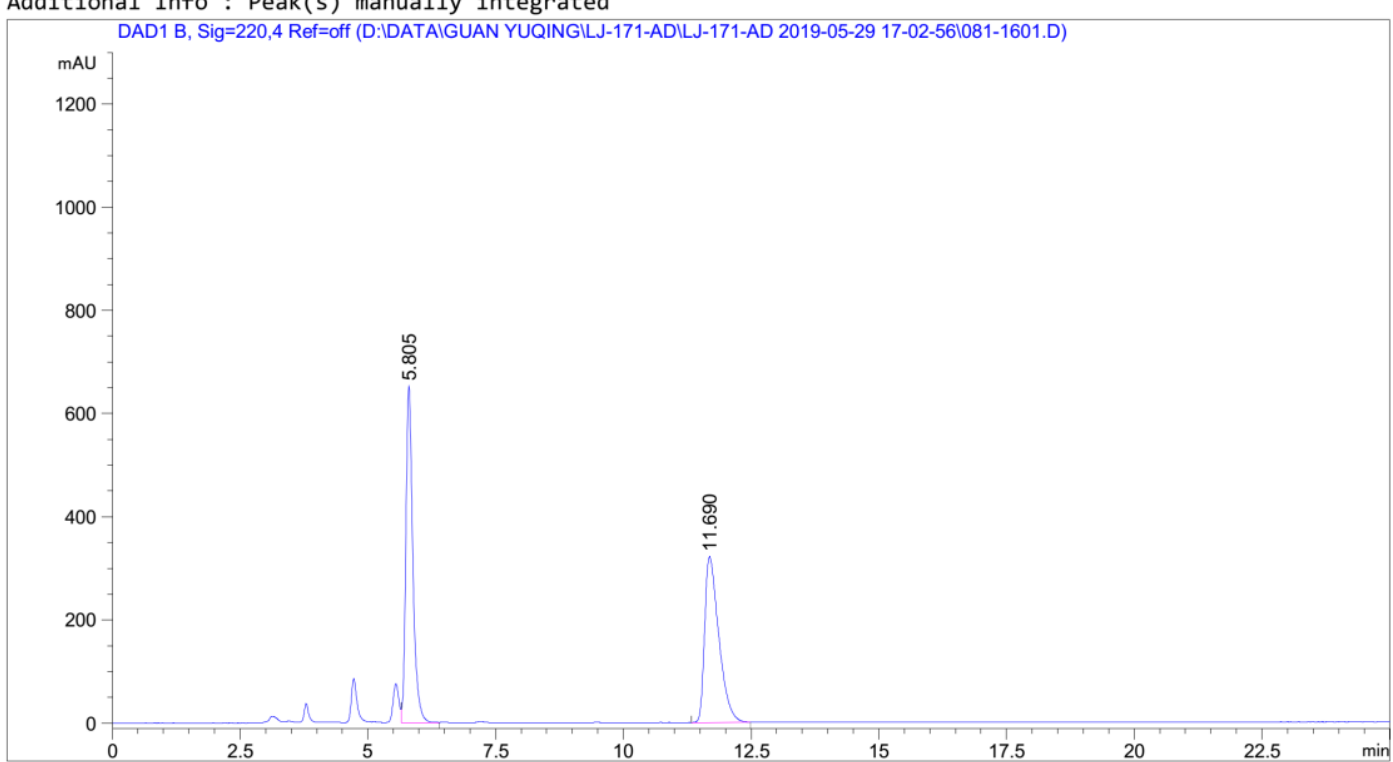

Area Percent Report

$\begin{array}{lll}\text { Sorted By } & : & \text { Signal } \\ \text { Multiplier } & : & 1.0000 \\ \text { Dilution } & : & 1.0000\end{array}$

Use Multiplier \& Dilution Factor with ISTDs

Signal 1: DAD1 B, Sig=220,4 Ref=off

\begin{tabular}{|c|c|c|c|c|c|}
\hline $\begin{array}{c}\text { Peak } \\
\#\end{array}$ & $\begin{array}{l}\text { RetTime Type } \\
\text { [min] }\end{array}$ & $\begin{array}{l}\text { Width } \\
\text { [min] }\end{array}$ & $\begin{array}{c}\text { Area } \\
{\left[\mathrm{mAU}^{*} \mathrm{~s}\right]}\end{array}$ & $\begin{array}{l}\text { Height } \\
\text { [mAU] }\end{array}$ & $\begin{array}{c}\text { Area } \\
\%\end{array}$ \\
\hline$-\ldots$ & $-\cdots---$ & - & |-..... & - & - \\
\hline 1 & $5.805 \mathrm{VB}$ & 0.1423 & 6170.86377 & 652.65344 & 49.9608 \\
\hline 2 & $11.690 \mathrm{BV}$ & 0.2903 & 6180.55566 & 321.54147 & 50.0392 \\
\hline Tot & : & & $1.23514 \mathrm{e} 4$ & 974.19492 & \\
\hline
\end{tabular}




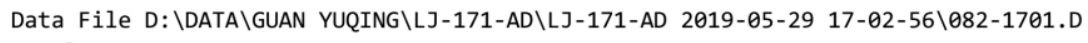
Sample Name: LSL-4-61
Acq. Operator
Acq. Instrument : Instrument 2
Seq. Line : 17
Location : Vial 82
Injection Date : 5/29/2019 11:33:13 PM
Inj : 1
Inj Volume : $5.000 \mu \mathrm{l}$

Acq. Method : D: \DATA \GUAN YUQING \LJ-171-AD \LJ-171-AD 2019-05-29 17-02-56\DAD-OD(1-6)-982-1ML-5UL-ALL-4OMIN.M

Last changed : 5/25/2019 3:07:40 PM

Analysis Method: D: \METHOD\LWD\DAD-AD(1-6)-95-5-1ML-3UL-ALL-30MIN-0813.M

Last changed : 8/13/2019 8:07:14 PM

(modified after loading)

Additional Info : Peak(s) manually integrated

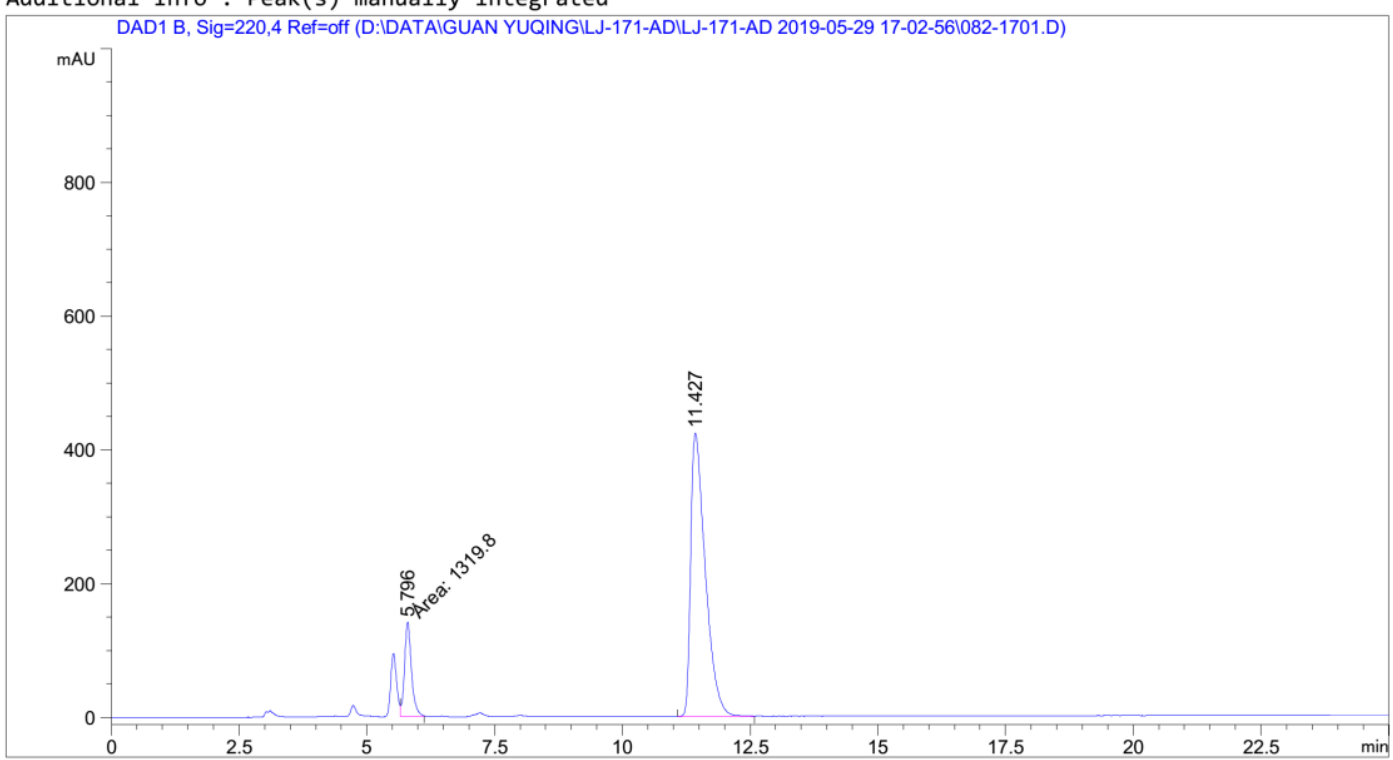

Area Percent Report

$\begin{array}{lll}\text { Sorted By } & : & \text { Signal } \\ \text { Multiplier } & : & 1.0000 \\ \text { Dilution } & : & 1.0000\end{array}$

Use Multiplier \& Dilution Factor with ISTDs

Signal 1: DAD1 B, Sig=220,4 Ref=off

\begin{tabular}{|c|c|c|c|c|c|}
\hline $\begin{array}{c}\text { Peak } \\
\#\end{array}$ & $\begin{array}{l}\text { RetTime Type } \\
\text { [min] }\end{array}$ & $\begin{array}{l}\text { Width } \\
\text { [min] }\end{array}$ & $\begin{array}{c}\text { Area } \\
{\left[\mathrm{mAU}^{*} \mathrm{~s}\right]}\end{array}$ & $\begin{array}{l}\text { Height } \\
\text { [mAU] }\end{array}$ & $\begin{array}{c}\text { Area } \\
\%\end{array}$ \\
\hline$-\ldots$ & - & (..... & |-..... & - & | \\
\hline 1 & $5.796 \mathrm{FM}$ & 0.1558 & 1319.80078 & 141.18221 & 13.5841 \\
\hline 2 & $11.427 \mathrm{BB}$ & 0.2933 & 8395.98242 & 423.57257 & 86.4159 \\
\hline Tot & : & & 9715.78320 & 564.75478 & \\
\hline
\end{tabular}




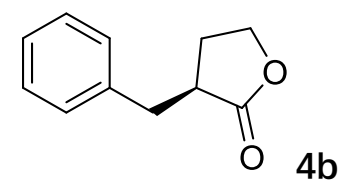

Data File D: \DATA \LSL\LSL-4-101\LSL-4-101 2019-07-14 22-04-06\003-0301.D Sample Name: LSL-4-101-RAC

\begin{tabular}{|c|c|c|}
\hline Acq. Operator & $:$ & Seq. Line : 3 \\
\hline Acq. Instrument & : Instrument 2 & Location : Vial 3 \\
\hline Injection Date & : 7/14/2019 10:27:20 PM & $\begin{array}{rcc}\text { Inj } & : & 1 \\
\text { Inj Volume } & : & 5.000\end{array}$ \\
\hline
\end{tabular}

Acq. Method : D: \DATA \LSL\LSL-4-101\LSL-4-101 2019-07-14 22-04-06\DAD-OD(1-2)-98-2-1ML5UL-ALL-40MIN.M

Last changed : 7/14/2019 10:52:50 PM

(modified after loading)

Analysis Method: D: \METHOD \LYH\DAD-OD(1-2)-98-2-0.5ML-5UL-ALL-60MIN.M

Last changed : 7/15/2019 8:44:13 AM

(modified after loading)

Additional Info : Peak(s) manually integrated

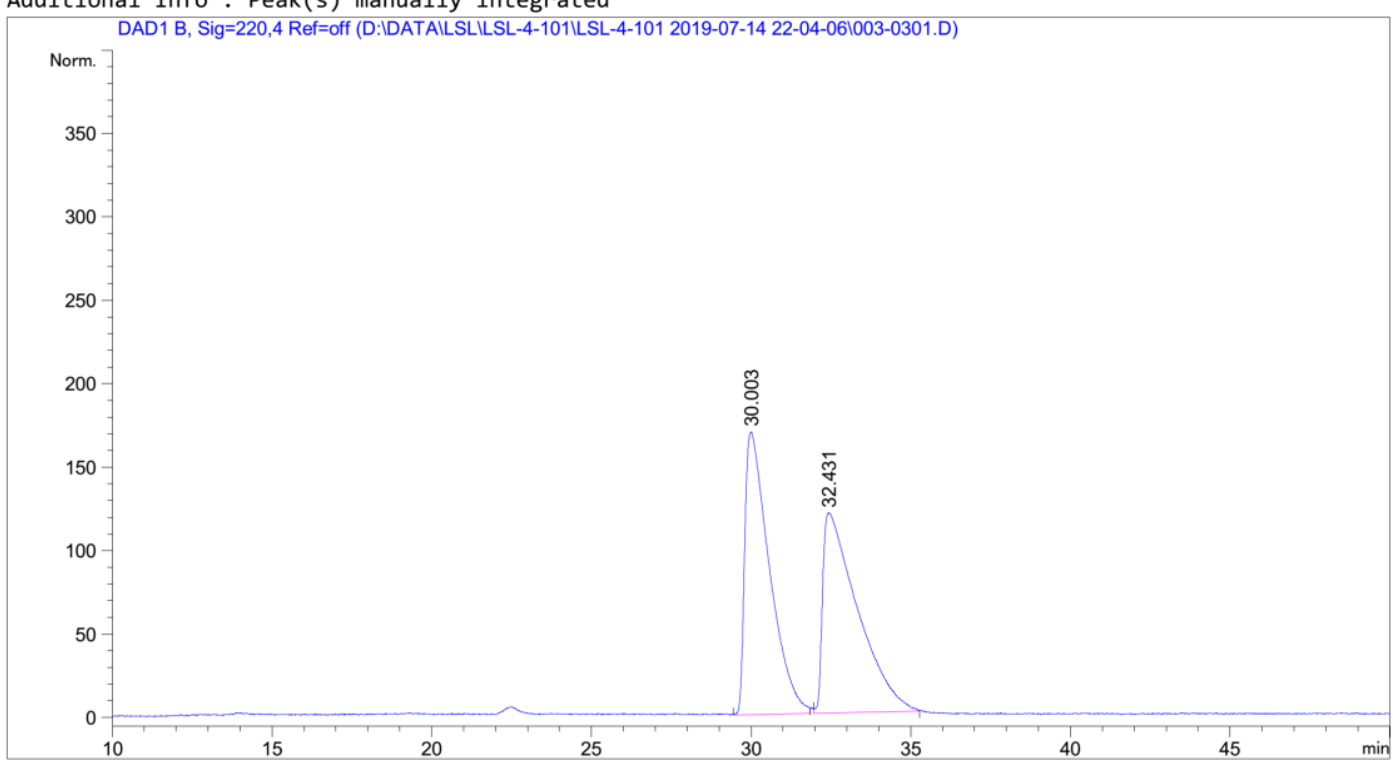

Area Percent Report

Sorted By : : Signal

Multiplier $\quad: \quad 1.0000$

Dilution : 1.0000

Use Multiplier \& Dilution Factor with ISTDs

Signal 1: DAD1 B, Sig=220,4 Ref=off

\begin{tabular}{|c|c|c|c|c|c|}
\hline $\begin{array}{c}\text { Peak } \\
\#\end{array}$ & $\begin{array}{l}\text { RetTime Type } \\
\text { [min] }\end{array}$ & $\begin{array}{l}\text { Width } \\
\text { [min] }\end{array}$ & $\begin{array}{c}\text { Area } \\
{[\mathrm{mAU} * \mathrm{~s}]}\end{array}$ & $\begin{array}{l}\text { Height } \\
\text { [mAU] }\end{array}$ & $\begin{array}{c}\text { Area } \\
\%\end{array}$ \\
\hline & & & & & can \\
\hline 1 & $30.003 \mathrm{VV}$ & 0.6380 & 9079.20801 & 169.20441 & 50.0814 \\
\hline 2 & $32.431 \mathrm{VB}$ & 0.8874 & 9049.70410 & 120.05280 & 49.9186 \\
\hline Tota. & s : & & $1.81289 \mathrm{e} 4$ & 289.25721 & \\
\hline
\end{tabular}


Data File D: \DATA \LSL\LSL-4-101\LSL-4-101 2019-07-14 22-04-06\004-0401.D Sample Name: LSL-4-101
Acq. Operator
Acq. Instrument : Instrument 2
Seq. Line : 4
Location : Vial 4
Inj : 1
Injection Date : 7/14/2019 11:28:19 PM
Inj Volume : $5.000 \mu \mathrm{l}$

Acq. Method : D: \DATA \LSL\LSL-4-101\LSL-4-101 2019-07-14 22-04-06\DAD-OD(1-2)-98-2-1ML5UL-ALL-4OMIN.M

Last changed : 7/14/2019 10:52:50 PM

(modified after loading)

Analysis Method : D: \METHOD \LYH\DAD-OD(1-2)-98-2-0.5ML-5UL-ALL-60MIN.M

Last changed : 7/15/2019 8:44:13 AM

(modified after loading)

Additional Info : Peak(s) manually integrated

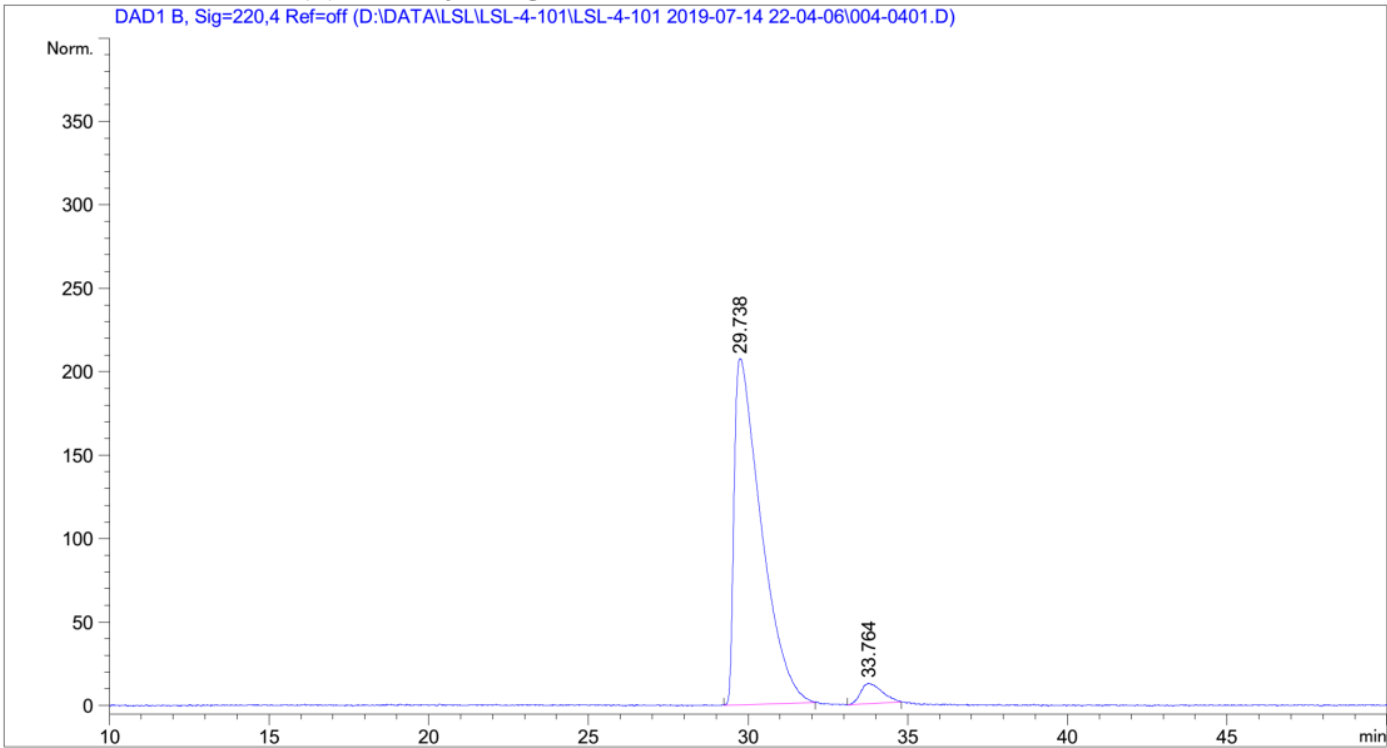

Area Percent Report

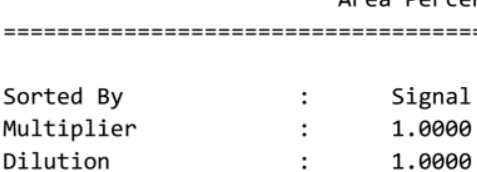

Use Multiplier \& Dilution Factor with ISTD

Signal 1: DAD1 B, Sig=220,4 Ref=off

\begin{tabular}{|c|c|c|c|c|c|}
\hline $\begin{array}{c}\text { Peak } \\
\#\end{array}$ & $\begin{array}{l}\text { RetTime Type } \\
\text { [min] }\end{array}$ & $\begin{array}{l}\text { Width } \\
\text { [min] }\end{array}$ & $\begin{array}{c}\text { Area } \\
{[\mathrm{mAU} * \mathrm{~s}]}\end{array}$ & $\begin{array}{l}\text { Height } \\
\text { [mAU] }\end{array}$ & $\begin{array}{c}\text { Area } \\
\%\end{array}$ \\
\hline 1 & 29.738 VB & 0. 7459 & $1.22027 e 4$ & 207.57225 & 95.5289 \\
\hline 2 & $33.764 \mathrm{BB}$ & 0.5533 & 571.13190 & 12.26329 & 4.4711 \\
\hline Tota & : & & $1.27738 \mathrm{e} 4$ & 219.83554 & \\
\hline
\end{tabular}

